FACULTAD DE CIENCIAS

Departamento de Física Aplicada. Área de Óptica

\title{
Optical Parametric Processes with Femtosecond Pulses in Nonlinear Crystals. Novel Schemes and Applications
}

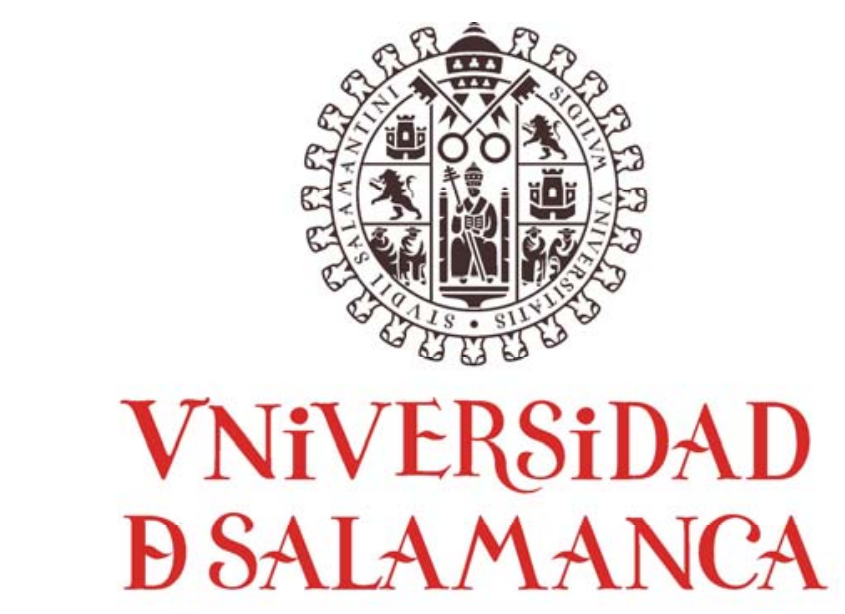

CAMPUS DE EXCELENCIA INTERNACIONAL

Tesis Doctoral

Carolina Romero Vázquez

Director: Javier Rodríguez Vázquez de Aldana

Salamanca, Enero 2012 

Cando penso que te fuches, negra sombra que me asombras, ó pé dos meus cabezales tornas facéndome mofa.

Cando maxino que es ida, no mesmo sol te me amostras, $i$ eres a estrela que brila, $i$ eres o vento que zoa.

Si cantan, es ti que cantas, si choran, es ti que choras, i es o marmurio do río $i$ es a noite i es a aurora. En todo estás e ti es todo, pra min i en min mesma moras,

nin me abandonarás nunca, sombra que sempre me asombras

Negra Sombra

Rosalía de Castro 



\section{Agradecimientos}

Las ciencias tienen las raices amargas, pero muy dulces los frutos.

(Aristóteles)

Parece que por fin esta aventura va llegando a su fin y sin duda habría sido imposible llegar hasta aquí sin la ayuda de todos los que habéis estado a mi lado y que espero encontréis en estas palabras mi reconocimiento.

Quiero en primer lugar dar las gracias mi director, Javi, sin su ayuda no estaría aquí escribiendo estas líneas, que además se quedan cortas para expresar todo lo que en realidad le debo. Le agradezco, sobre todo, la paciencia que ha tenido conmigo, en estos ya más de cuatros años, resolviendo todas mis dudas y en ciertos temas lagunas. Ha sido un placer trabajar contigo todo este tiempo, no se me ocurre que pudiera tener un director mejor: "la perfecta simbiosis de físico teórico y experimental". Las palabras no son mías, pero reflejan fielmente mi opinión, así que me he tomado la libertad de reproducirlas aquí. Solamente espero que algo de su buen hacer se me haya pegado. ¡Muchísimas gracias jefe!

Quiero agradecer también a todo el Fotón Charro a los que están y los que estuvieron, por la forma en la que me acogieron desde mi llegada como alumna del máster. Han hecho que estos años de tesis fueran una gran experiencia para mí.

A Luis Roso le agradezco que confiara en mí y me diera la oportunidad de comenzar esta aventura concediéndome la beca: sin ese soporte seguramente no hubiera tenido un fin. A Luis Plaja, quizá sin quererlo, el primer responsable de todo esto, por haber contestado aquel e-mail en agosto de 2006. Le agradezco muchas risas y conversaciones, a veces un poco filosóficas.

A Isabel Arias le agradezco muchas cosas, entre ellas presionarme para escribir, lo que me ha costado,... pero sobre todo que me animó a venir a Salamanca, aún recuerdo sus palabas “ ¡Venga, los gallegos para Salamanca!" y aquí seguimos.

A Cruz, que me enseñó a moverme en el laboratorio, eso y mucho más, espero haber tomado buena nota de todo. También por los buenos ratos que pasamos juntas, sobre todo al principio cuando eras mi compañera de mesa. Como dicen en mi tierra: "xa choveu".

A Pablo....jay Pablo!, tengo que decirte que es todo un orgullo para mí ser "la luz de la mañana", muchas gracias por sacarme siempre una sonrisa, sobre todo en esta última temporada con la escritura que tanto lo necesitaba. Supongo que echarás de menos mis visitas al procesado, iyo seguro que sí!, porque no tendré adonde ir cuando quiera "dispersarme". También gracias por las tertulias de los viernes, y por los cafés en el Corral, principalmente por las risas y los debates que nos ayudan a desconectar del trabajo, pero sobre todo por hacerme sentir princesa entre tanto "buitre" (perdón por lo de buitres, chicos, pero ¡lo tenía que poner!). 
A los demás compañeros, Iñigo, Jose, Julio, Camilo R., Enrique, Ana, Ricardo,... igracias por estar ahí siempre cuando necesitaba algo y, sobre todo, por hacerme sentir como en casa desde el principio!

A Iñigo, Jose y Viqui les quiero agradecer además por ser mi apoyo en los primeros meses en el grupo y también por los consejos recibidos sobre todo este proceso de convertirse en doctor/ra. Jose, eres el siguiente, ¡mucha suerte!

Al equipo técnico: Javi Santa, Juan, Camilo P. e Isa, muchas gracias por la ayuda y soporte en el laboratorio, los becarios sin vosotros no somos nada. También gracias por animarme durante estos años, sobre todo en esta recta final que parece que no acaba.

Bueno Cris, creo que esto significa que ya nos queda menos para la noche de chicas, y tarde de compras,.. ¡o lo que nos echen! Gracias también por tu apoyo.

Al "núcleo duro de la OSAL": Rocío, Carlos y Benjamín, gracias por embarcarme en esas aventuras en las que tanto hemos sufrido por un lado, pero que creo nos han ayudado a todos mucho, sobre todo a movernos en ciertos "mundillos" y situaciones en la vida tanto personal como profesional. He sentido especialmente vuestro apoyo, sobre todo en esta última etapa, así que espero de alguna manera poder devolvéroslo y desde aquí, toda mi fuerza para en este año que os espera, os deseo lo mejor para vuestras tesis. A los demás compañeros becarios: Warein, Alexis, Camilo G., Alejando, Fran también mucha suerte con las vuestras.

A mis niñas de Baiona, creo que pronto dejaré de "pegar la chaqueta con la tesis". Gracias por los ánimos que me habéis dado, ¡sois las mejores! En especial a Maru le quiero agradecer los años que pasamos en Santiago, en los que me has ayudado tanto. Iria, ha sido muy especial pasar esta etapa de nuestra vida juntas, al igual que otras ya pasadas, creo que mutuamente nos hemos comprendido y ayudado. Muchas gracias por estar siempre ahí.

A mi familia, mis padres, hermanas y abuelas, por que sin su apoyo no hubiera podido llegar hasta aquí, ni a muchos otros sitios en el camino. Les agradezco sobre todo la confianza que siempre han tenido en mí, sin duda más que yo misma.

A mi Óscar, igracias por todo! pero sobre todo por animarme en los días duros: espero que esta aventura sea solamente la primera de muchas que vivamos juntos. 


\section{Acronyms List}

CPA: Chirped Pulse Amplification

DFG: Difference-Frequency Generation

DL: Diffractive Lens

FROG: Frequency Resolved Optical Gating

FWHM: Full Width Half Maximum

GDD: Group-Delay Dispersion

GVD: Group-Velocity Dispersion

GVM: Group-Velocity Mismatch

HHG: High-Harmonic Generation

M-L: Mode-Locking

NA: Numerical Aperture

NOPA: Noncollinear Optical Parametric Amplifier

OPA: Optical Parametric Amplification

OPCPA: Optical Parametric Chirped Pulse Amplification

OPG: Optical Parametric Generation

OPO: Optical Parametric Oscillator

SC: Supercontinuum

SFG: Sum-Frequency Generation

SFM: Self-phase Modulation

SHG: Second-Harmonic Generation

SVEA: Slowly Varying Amplitude Approximation

THG: Third-Harmonic Generation

X-FROG: Cross- Frequency Resolved Optical Gating 



\section{Contents}

Agradecimientos ........................................................................................................................................ i

Acronyms List .........................................................................................................................iii

Chapter 1 .......................................................................................................................................... 5

A Background to Ultrafast and Ultraintense Lasers: Technology and Applications ................... 5

1.1. Introduction: More than 50 Years of Laser Technology ................................................. 5

1.2. Ultrashort Laser Pulses Generation Techniques: Q-Switching and Mode-Locking ............... 6

1.3. The Chirped Pulse Amplification Technique ...................................................................... 7

1.3.1. The Laser Systems in the Laboratory of Salamanca ..................................................... 9

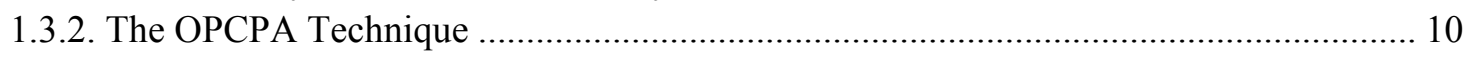

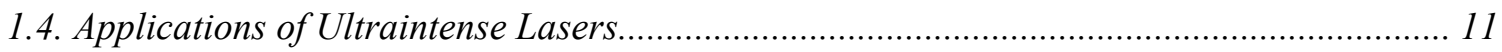

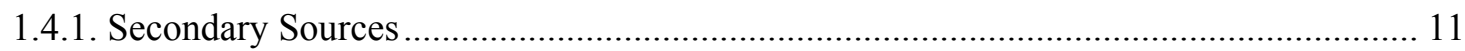

1.4.2. Application of Ultraintense Lasers to Materials Micro-Processing ................................ 12

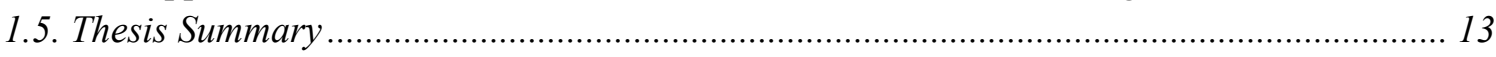

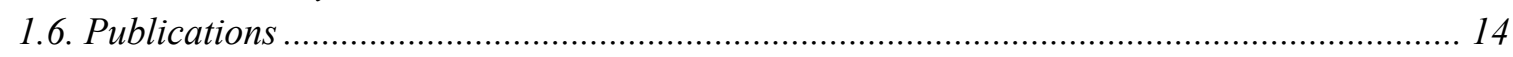

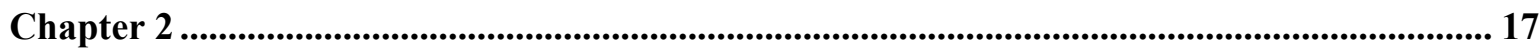

Introduction to Nonlinear Optics: Parametric Processes ............................................................. 17

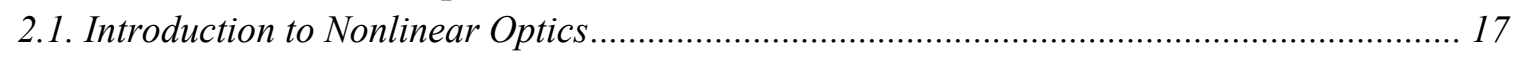

2.2. Microscopic Classical Model of the Light-Matter Interaction .............................................. 20

2.3. The Wave Equation for Nonlinear Optical Media .............................................................. 23

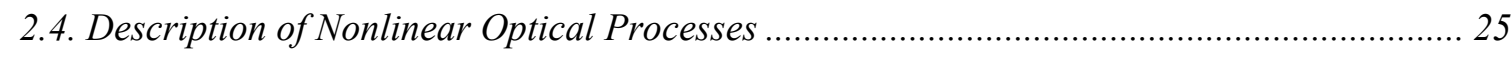

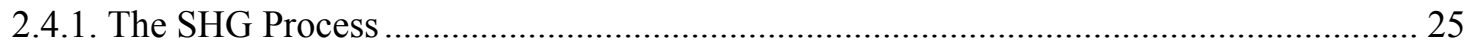

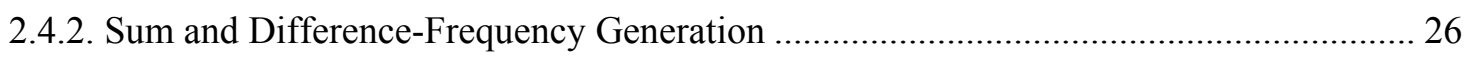

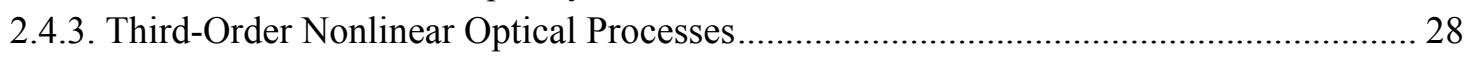

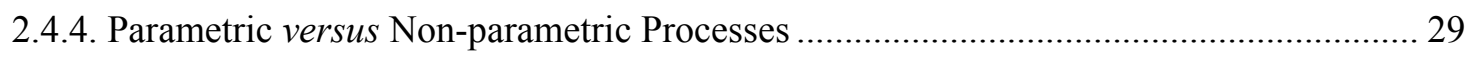

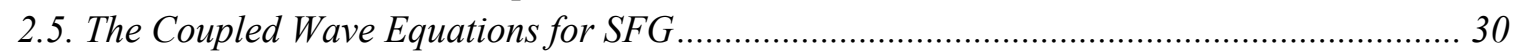

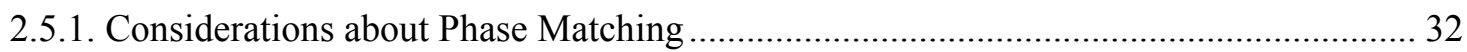

2.5.2. Solutions Beyond the Undepleted Pump Approximation ............................................. 33

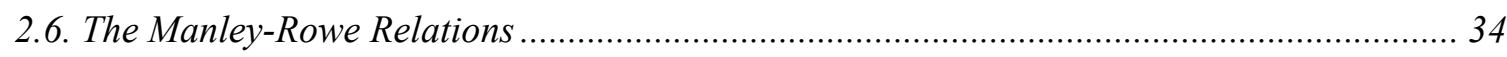

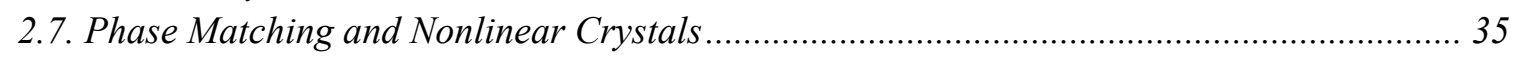




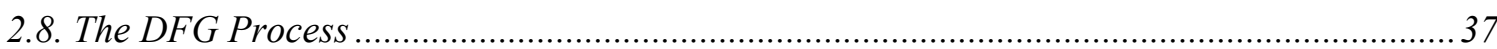

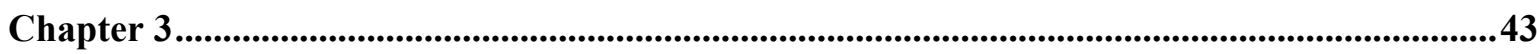

Design, Characterization and Implementation of a Femtosecond SHG/THG Module..............43

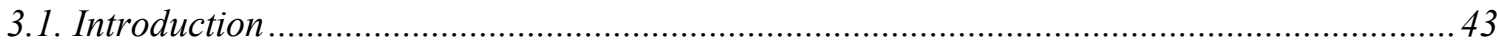

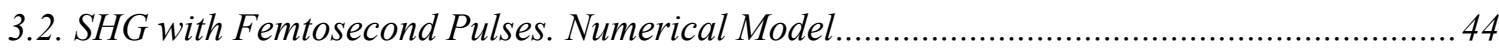

3.3. Temporal Characteristics of the Second Harmonic Generated with Femtosecond Pulses.... 46

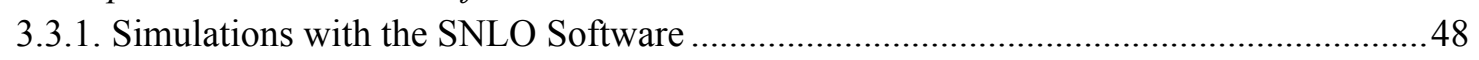

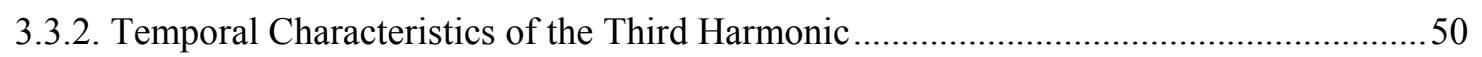

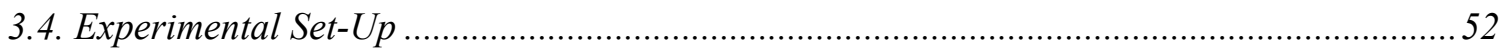

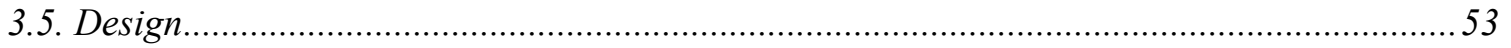

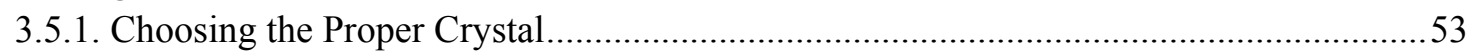

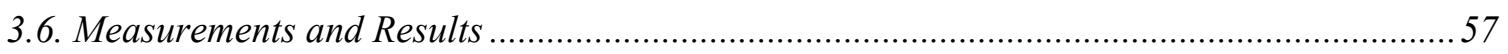

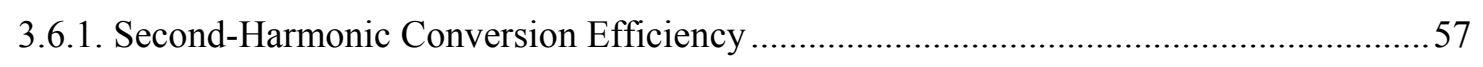

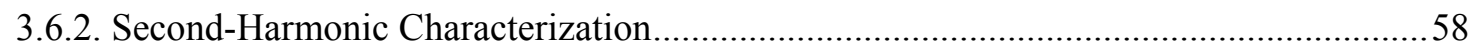

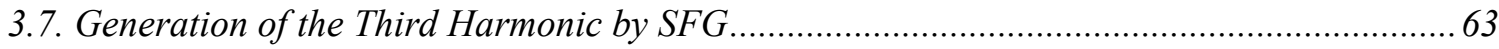

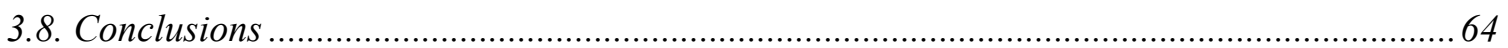

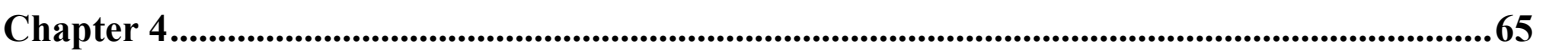

Third Harmonic Generation in a Micro-Structured BBO Crystal.........................................65

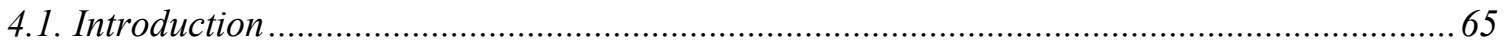

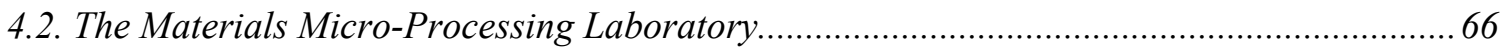

4.3. The Micro-Structured Crystal and the Grating Characterization.........................................68

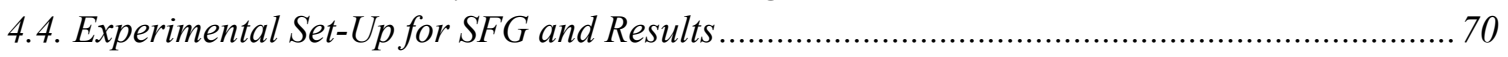

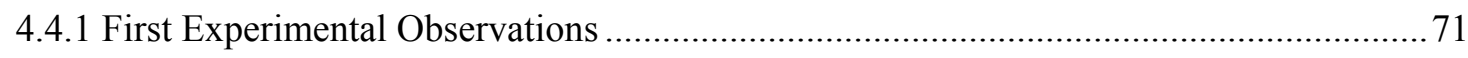

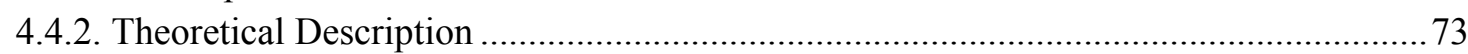

4.5. Application of the Micro-Structured BBO to Intensity Cross-Correlation Measurements .... 76

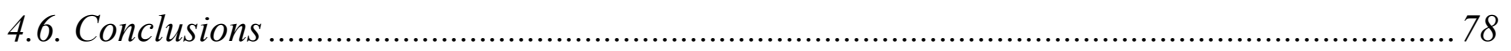

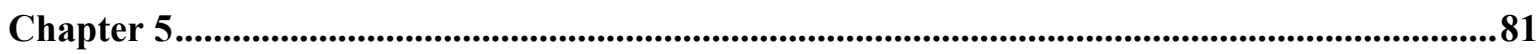

Second- and Third-Harmonic Generation with Diffractive Lenses.........................................81

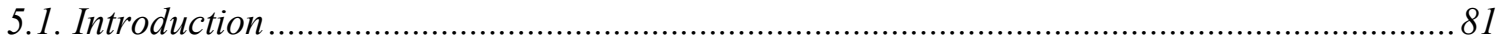

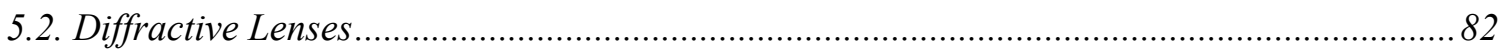

5.3. Wavelength Tunability in the Second-Harmonic Signal. Study of the Influence of Crystal

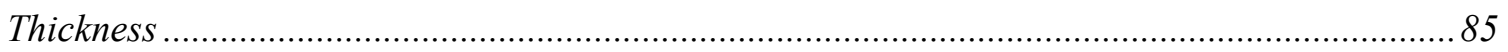

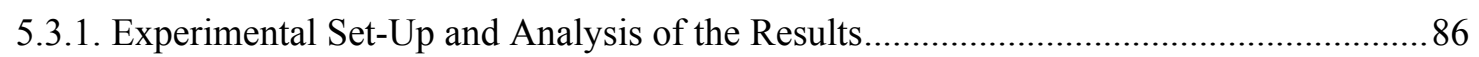

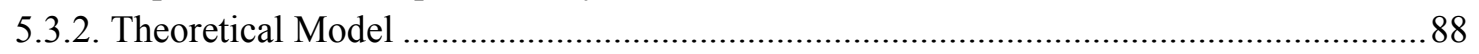

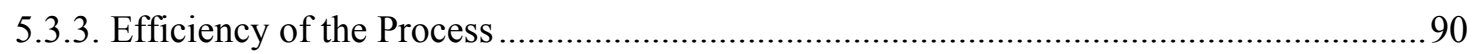

5.4. Characterization of the Second Harmonic Generated with a DL ......................................... 91

5.4.1. Temporal and Spectral Characterization: X-FROG Measurements ................................ 91

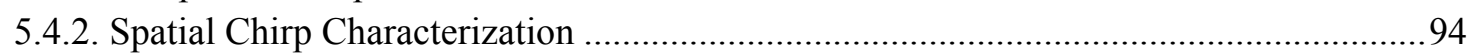

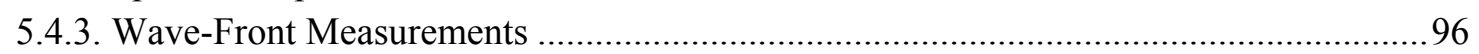

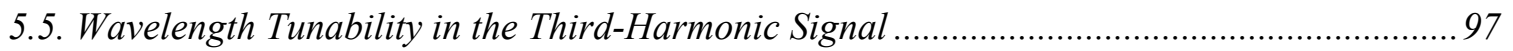

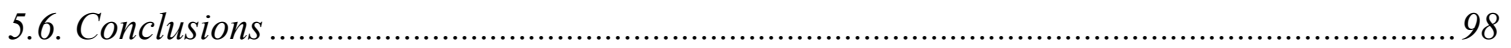

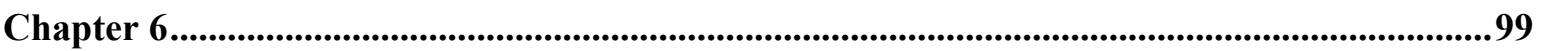

Supercontinuum Generation in Bulk Media with Diffractive Lenses ........................................99 


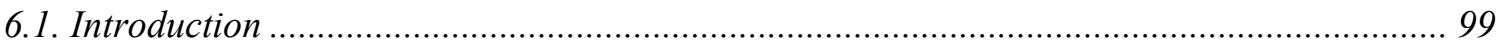

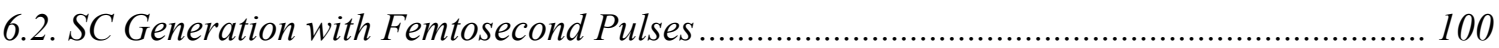

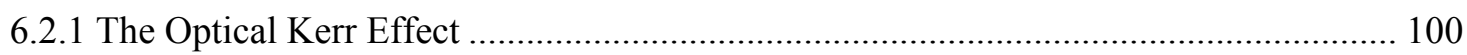

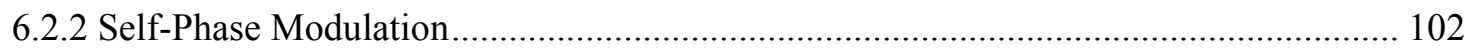

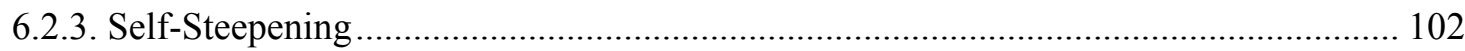

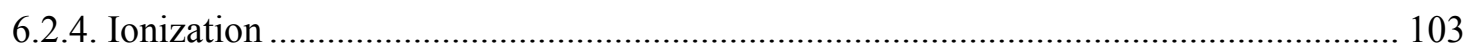

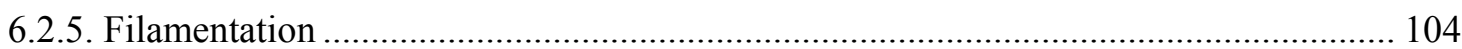

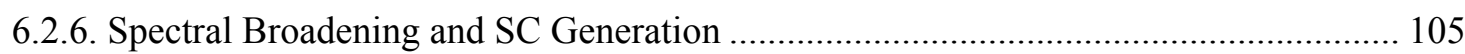

6.3. Experimental Arrangement for the Generation of SC with a DL .................................... 106

6.4. SC Generation in Sapphire. Experimental Measurements and Analysis of the Results ...... 108

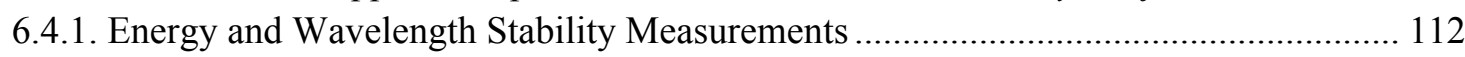

6.4.2. Dependence of the SC on Parameters of the Input Beam: NA and Energy .................. 114

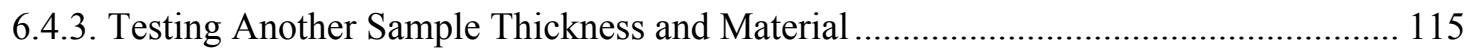

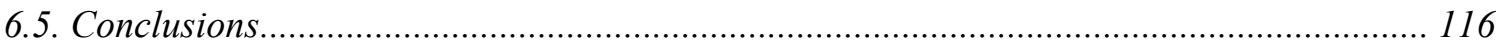

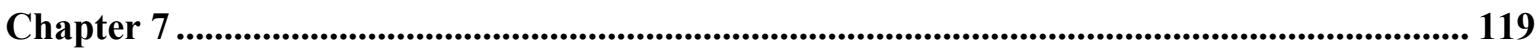

Design and Implementation of a Noncollinear OPA in the Visible for fs Pulses...................... 119

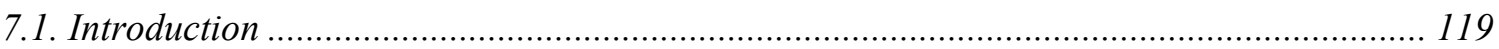

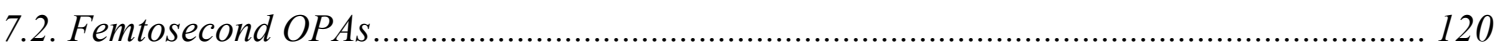

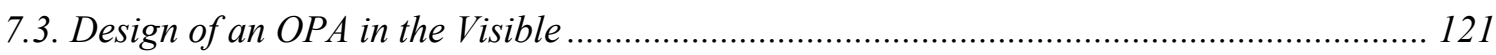

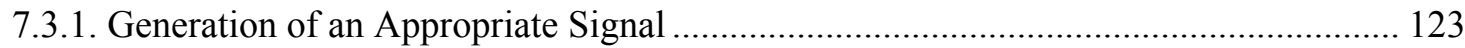

7.4. Optical Parametric Amplification with Femtosecond Pulses: Theoretical Model .............. 124

7.4.1. Noncollinear Phase Matching: Broadband Amplification ............................................ 127

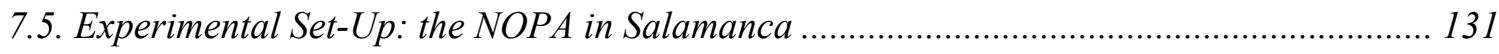

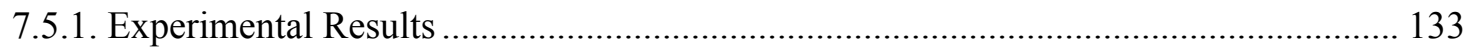

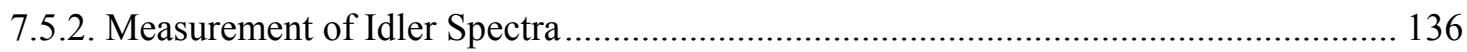

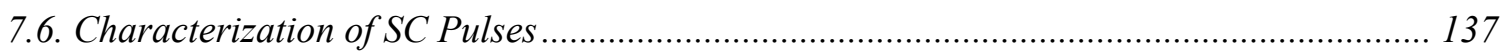

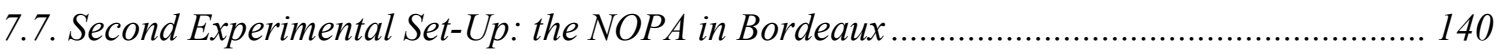

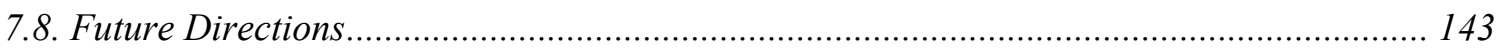

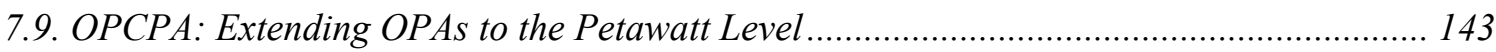

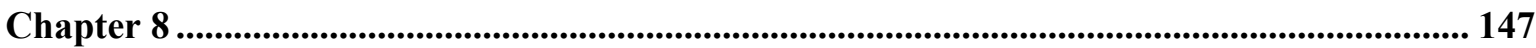

Materials Micro-Processing with Vis-UV Femtosecond Pulses: Applications ........................ 147

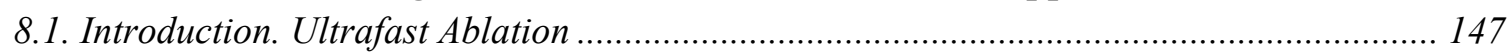

8.2. Effect of Wavelength in Femtosecond Micro-Processing …............................................ 148

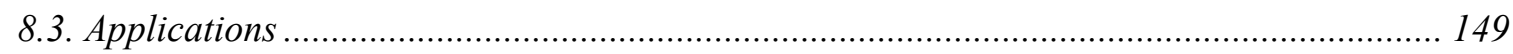

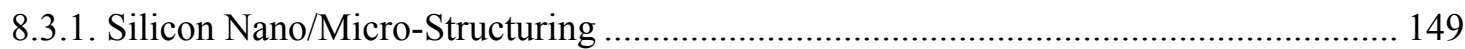

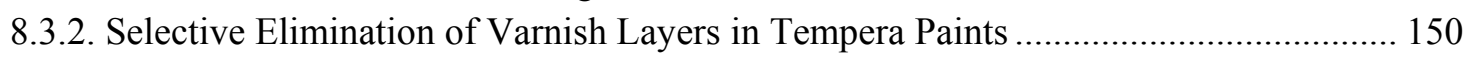

8.3.3. Fabrication of Optical Waveguides in Active Crystals.............................................. 152

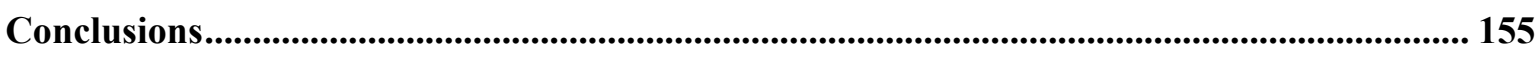

Appendix A..................................................................................................................................... 159

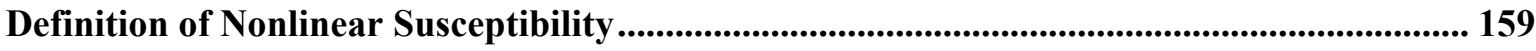

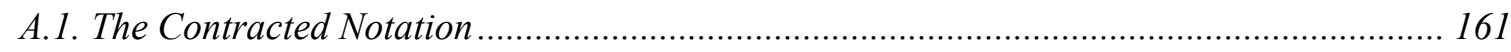

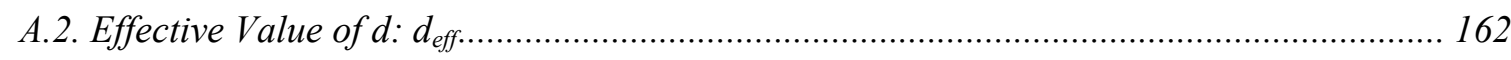

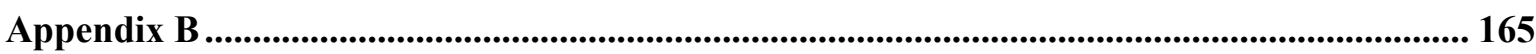




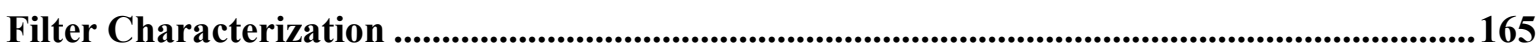

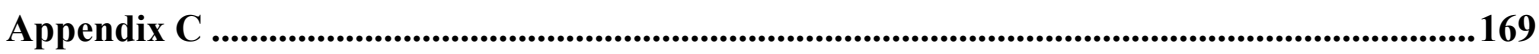

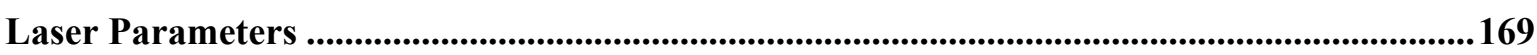

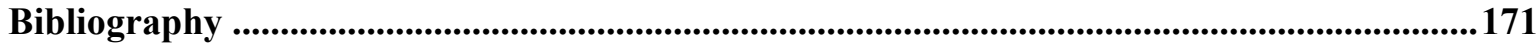




\section{Chapter 1}

\section{A Background to Ultrafast and Ultraintense Lasers: Technology and Applications}

\subsection{Introduction: More than 50 Years of Laser Technology}

Since the first laser became operative in 1960 [1] lasers have contributed decisively to the development of many scientific and technological areas. Owing to their favourable properties, lasers have become essential tools in medicine, have revolutionized optical communications, and have been applied in many industrial processes. What is also fascinating is that, unlike other technological advances, lasers are present all around us in our daily lives, from the CD player to the bar code scanner. Moreover, active laser-related scientific research has seen 9 Nobel prizes awarded in the past decade [2], related either to laser development itself or to the scientific advances that have been made possible thanks to them. Moreover this laser-related research is still a very active field and it is expected that the development of new laser sources will lead to important scientific discoveries in the near future.

Concerning nonlinear optics, its beginning it is considered to have occurred just one year after the demonstration of the first laser. The observation that gave rise to the birth of this new field was the experimental proof of the generation of the second harmonic from a ruby laser, using a quartz crystal [3]. The second harmonic is the generation of light with twice the frequency of the original from the laser. Although the phenomenon had been predicted before, it was not until the discovery of the laser that it could be demonstrated. The new light source could provide an electric field comparable in amplitude to the inter-atomic field that was needed to observe experimentally the phenomenon. Fifty years later, this process is still of great importance. In fact, it is used to generate 
the radiation that pumps the amplification stages in large-scale Petawatt $\left(\mathrm{PW}, 10^{15} \mathrm{~W}\right)$ peak power lasers.

Naturally, over the years of technological development in lasers many different types have emerged, ranging from small semiconductor-based lasers, millimetres in size, to "huge" very energetic lasers that take up much more space than a room, such as at the National Ignition Facility (NIF) in Livermore, California [4]. This is the world's largest and most energetic laser, but that's another story, far from the one in which we are interested.

Nevertheless, the development of laser technology itself is far from being over and, in contrast, is a very active field of research. In this thesis, we shall focus on ultrashort and ultraintense lasers. As can be expected, even within this community there are different sections. We have been using a laser with moderate energy, and although ultrashort, it is far from the limit of the few-cycle regime $^{1}$. By "moderate", we refer to energies per pulse up to $\sim 1 \mathrm{~mJ}$, and pulse lengths in the 100 femtoseconds ( $\mathrm{fs}, 10^{-15} \mathrm{~s}$ ) range, such that the peak power is in the order of GW. We have made this distinction because the development of this kind of laser is a very active line of research and peak powers can currently reach the PW level [5]. In what follows, we shall briefly review the benchmarks that have led to the birth of ultrafast and ultraintense laser technology [6].

\subsection{Ultrashort Laser Pulses Generation Techniques: Q-Switching and Mode-Locking}

Ruby lasers had the ability to produce light pulses, but in fact they were no more than uncontrolled bursts of radiation. However, not very long after the discovery of the laser, actually just two years later, the Q-Switching technique was demonstrated [7]. Higher energies and shorter pulses could be generated by altering the Q (quality) factor of the laser cavity. Briefly, Q-switching is based on storing the energy from the pump beam in terms of population inversion. With external control, radiation losses are applied in the laser cavity (decreasing the Q-factor) while the energy from the pump increases the population inversion. Then, at some point the Q-factor of the cavity is changed to a high-value (reducing losses) and the energy is delivered in a short single pulse. Usually, this is done with an electronic device (active techniques) and the time duration of these pulses is in the range of ns; no further reduction can be achieved with this method.

The next technique to be developed, namely Mode-Locking (M-L), offers the possibility of further reducing the temporal extent of the pulses, and this became the basis for femtosecondpulsed laser development. The idea underlying M-L is to fix -by some method- the relative phases of the modes inside the laser cavity, which naturally oscillate with random phases. When these modes are in phase, the intra-cavity intensity will be increased. Therefore, by creating an intensitydependent gain in the cavity amplification will occur only in the modes that are in phase. Over many passes in the gain medium, all the energy will be transferred to the set of modes with the same phase. This creates a train of pulses spaced by the round-trip cavity time $\tau=2 L / c, L$ being the cavity length and $c$ the speed of light in a vacuum. The bandwidth of a single pulse is $\Delta \omega=N \pi c / L$, where $N$ is the number of modes that undergo gain, limited (among other things) by the gain bandwidth of the active medium. Therefore, the duration of the pulses depends inversely on the

\footnotetext{
${ }^{1}$ A few-cycle laser pulse is such that the time duration of its intensity envelope corresponds to just a few periods of the carrier oscillation. For example in pulses centred at $\sim 800 \mathrm{~nm}$ a two-cycle pulse corresponds to a temporal duration of $\sim 5.4 \mathrm{fs}$.
} 
"locked" modes. The intensity-dependent gain in the cavity can be introduced actively by temporally modulating the gain in the cavity (active M-L techniques), or passively, when the absorption or gain in the cavity is dependent on the intensity of the pulse itself (passive M-L techniques).

The M-L allowed further reductions in the pulse length to be obtained, and hence greater peak intensities. It was in 1963 that L. E. Hargrove et al. [8] reported the first demonstration of a M-L laser; in that case it was an He-Ne laser, with an acousto-optic modulator: i.e. active M-L. However, the birth of ultrafast optics is considered to have taken place in 1974, when E. P. Ippen and C. V. Shank demonstrated a sub-picosecond (ps, $10^{-12} \mathrm{~s}$ ) M-L continuous-wave dye laser [9] with a passive M-L technique.

Later, in 1982 a new laser material was discovered by P. F. Moulton, the Ti:Sapphire (Ti: $\left.\mathrm{Al}_{2} \mathrm{O}_{3}\right)$. This crystal replaced the dye lasers for tunable and ultrafast laser applications. In fact, owing to its very favourable properties, the new material [10] ushered the field of pulsed lasers into what can be called the Ti:Sapphire revolution.

One of the very good features of the Ti:Sapphire crystal is the fact that it has a very large gain bandwidth (from 650 to $1100 \mathrm{~nm}$ ) in comparison with other materials, allowing the generation of very short pulses. Recall that the pulse bandwidth that can be achieved with the M-L technique depends on the medium's gain bandwidth. Once the Ti:Sapphire crystal had been discovered, powerful M-L techniques, such as Kerr-lens M-L [11] or the saturable absorber, emerged.

\subsection{The Chirped Pulse Amplification Technique}

Ultrafast science is a rapidly evolving multidisciplinary field: the ability to excite matter with femtosecond light pulses and to probe its subsequent evolution at ultrashort time scales opens up completely new fields of research in physics, chemistry and biology, because many processes of interest in these fields proceed at an ultrafast time scale.

Advances in laser physics experiments have been intimately linked to technological advances in laser development, because the developments in laser technology have opened up new regions in light-matter interaction for exploration. For many applications, femtosecond Ti:Sapphire lasers delivering pulses of few $\mathrm{nJ}$ are sufficient. The field of femtochemistry, for example, has found a major tool for research purposes in such lasers. However, other applications require higher intensities that can only be accomplished by increasing the energy per pulse.

Another landmark of ultrashort laser technology was the discovery in 1985 by D. Strickland and G. Mourou [12] of the Chirped Pulse Amplification (CPA) technique, which enabled the energy of femtosecond lasers to be increased by 2-3 orders of magnitude: from the $\mathrm{mJ}$ to the multi-J level. This technology was born as the solution for obtaining more energetic pulses from a Ti:Sapphire amplification system. The first limitation in laser amplifiers came from the damage threshold of the amplification crystals as well as of the optical components. The first attempt to overcome this drawback was to increase the beam section and insert crystals that were large enough to prevent the damage threshold from being reached.

This new technique, CPA, is based on the same idea that had previously been used to increase radar power, and is based on chromatic dispersion. The ultrashort pulses are stretched out in time, reducing peak power to a considerable extent, before amplification. Then, they are amplified to 
increase their energy (to high-energy but low-power) and compressed back to the original temporal duration. In fact, the term chirped comes from radar technology and it referred to a microwave pulse with a varying frequency in time. And this is how the pulses are stretched in time, with the introduction of chirp. For example, with the use of certain methods the redder frequencies are forced to travel shorter paths than the bluer wavelengths, so the reds will travel in the leading part of the pulse; i.e. the pulse has been positively chirped. In contrast, in the negative chirped pulse (i.e. negative dispersion) the longer wavelengths travel longer paths, and are therefore retarded with respect to the blue ones and are forced to propagate in the rear part of the pulse.

The essential parts of a CPA laser system are shown schematically in the Figure 1.1, the green arrows represents the pump lasers. In the case of the Ti:Sapphire crystal, these are Q-Switched doubled frequency solid-state lasers based on Nd-doped crystals (Nd:YAG, Nd:YVO, Nd:YLF and some others). The orange boxes represent the part of the system where laser amplification occurs; in the first case the orange box is the oscillator and in the second it represents an initial amplification stage. The term oscillator is reserved for a Ti:Sapphire laser that emits M-L pulses at low energy and a high repetition rate. The output of this laser serves as a seed for the next amplification stage, and so on. Pulses as short as $10 \mathrm{fs}$ can currently be obtained from Ti:Sapphirebased M-L oscillators at $100 \mathrm{MHz}$ repetition rates. The crystals in the laser amplifiers are also Ti:Sapphire owing to the favourable thermal and mechanical properties of this crystal, which supports high powers and intensities. Therefore, the material becomes important not only in the oscillators but also in the amplification stages, allowing powers as high as several watts for pulses shorter than 100 fs.

Finally, the blue boxes represent the parts of the system where the chirp of the pulses is changed. The pulses in the oscillator have widths below the picosecond regime and are stretched to pulse widths of hundreds of picoseconds or even nanoseconds. The stretching is obtained by means of strongly dispersive elements, normally a pair of gratings or prisms.

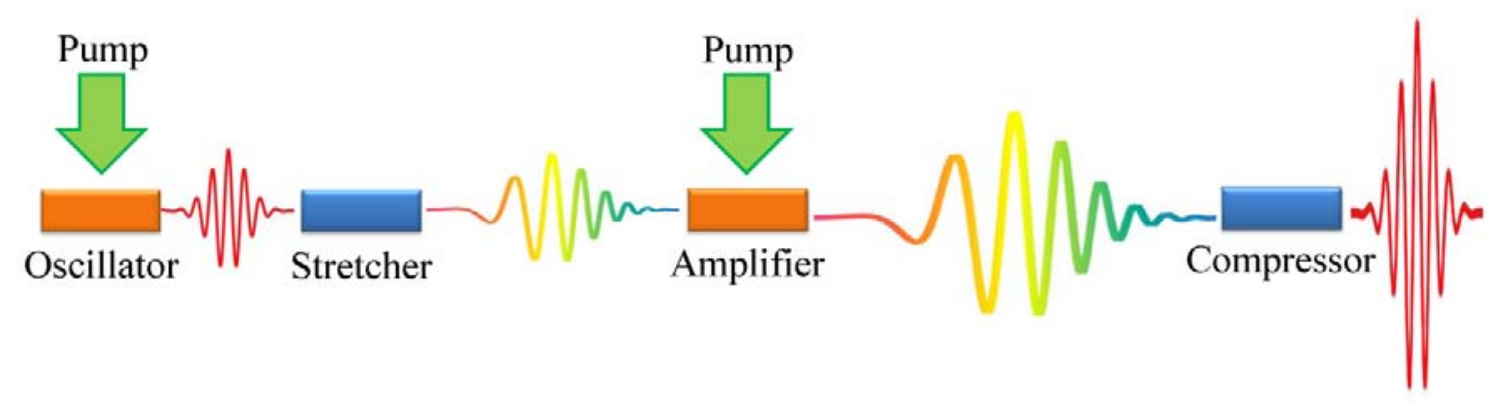

Figure 1.1. Conceptual scheme of the CPA technique.

After being stretched, the pulses can safely travel through the optics in the system as well as in the amplification crystals at low power. Finally, the chirp introduced in the stretcher is eliminated and the pulses are compressed back to their original time duration. The compression stages are all made with reflective optics. This set-up is dual: on the one hand, the damage thresholds can be higher than in refractive optics and, on the other, the amount of material that the ultrashort pulses have to travel through is reduced.

The peak powers obtained after the CPA stage are orders of magnitude higher than the pulses delivered by the Ti:Sapphire oscillator. The whole CPA system may consist of one or two amplifications stages, which can follow different strategies. The common issue is the fact that 
multiple passes in the amplification crystals are needed to obtain the required gain factor. The most common set-up to obtain very high gains is a regenerative amplifier [13]. In this case the gain medium, the Ti:Sapphire crystal, is placed in a optical resonator, with an optical switch (an electrooptics modulator: a Pockels cell and a linear polarizer) that allows the pulses to enter the resonator. Therefore, the number of round-trips in the resonator is controlled externally. It can be very large and hence the amplification factor (gain) can be huge. The other kind of amplification set-ups are the multipass amplifiers. The difference in this second kind is that the number of passes through the medium is fixed, not controlled by an optical switch, and instead is determined by the beam path's geometry. Accordingly, it is not a laser cavity as is the regenerative amplifier.

\subsubsection{The Laser Systems in the Laboratory of Salamanca}

The ultrafast ultraintense lasers laboratory in Salamanca began its operation in 2003 when the first CPA-based Ti:Sapphire laser system was installed, a system that continues operative today. In particular, it delivers pulses with a time duration of $\sim 120 \mathrm{fs}$ at the central wavelength typical of Ti:Sapphire, $795 \mathrm{~nm}$. It has the particularity of having two laser outputs; a scheme with the different elements can be seen in Figure 1.2. One of the outputs delivers low-energy pulses, at a high repetition rate, and the other -at low repetition rate- delivers high-energy pulses. In the first amplification stage, only one regenerative amplifier is used (Spitfire, Spectra-Physics) pumped by an Nd:YLF laser. One of each 100 pulses is further amplified in a multipass amplifier pumped by an Nd:YAG laser. In particular, the low-energy output delivers pulses of $\sim 1 \mathrm{~mJ}$ at $1 \mathrm{kHz}$ repetition rate, (peak power $\sim 9 \mathrm{GW}$ ) and the other with a repetition rate of $10 \mathrm{~Hz}$ and a pulse energy of $50 \mathrm{~mJ}$ has a peak power of 0.5 Terawatt $\left(\mathrm{TW}, 10^{12} \mathrm{~W}\right)$. This was the most intense laser in Spain until a new system arrived at Salamanca in 2008.

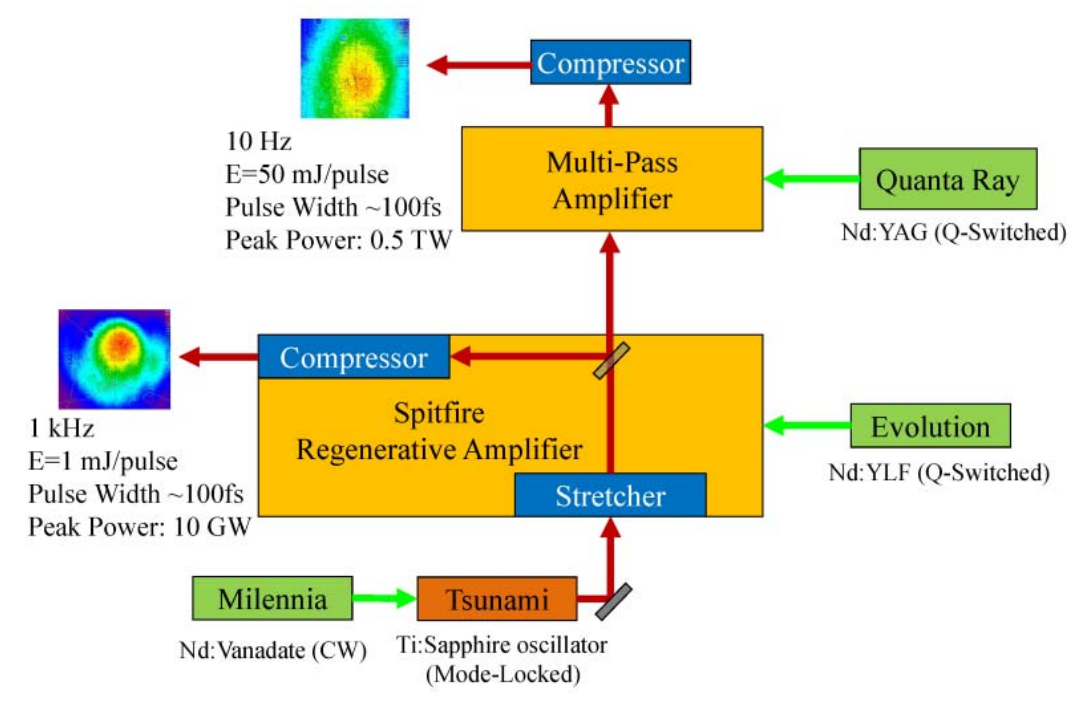

Figure 1.2. Scheme of the Spectra-Physics laser system at the laboratory of Salamanca.

The new laser system was also a commercial system from Amplitude Technologies. It had a peak power of $20 \mathrm{TW}$, with a pulse energy of $500 \mathrm{~mJ}$ and a pulse duration of $25 \mathrm{fs}$, at a repetition rate of $10 \mathrm{~Hz}$. The central wavelength was again that of Ti:Sapphire, and can be varied between 790 to $810 \mathrm{~nm}$. The technology was also CPA but with some improvements, such as a contrast booster, which eliminates pre- and post-pulses, a common problem in these more energetic systems. Currently, this laser is being integrated within a more powerful one that will deliver pulses of 200 
TW peak power, with an energy per pulse of $5 \mathrm{~J}$. This new laser system corresponds to the secondary phase of the CLPU [14]. The latter is the acronym for Centro de Láseres Pulsados Ultracortos Ultraintensos, which will soon become operative in Salamanca. It is expected that this will be a leading scientific infrastructure, with laser systems with peak powers of GW, TW and PW, that will be open to the domestic and international scientific community.

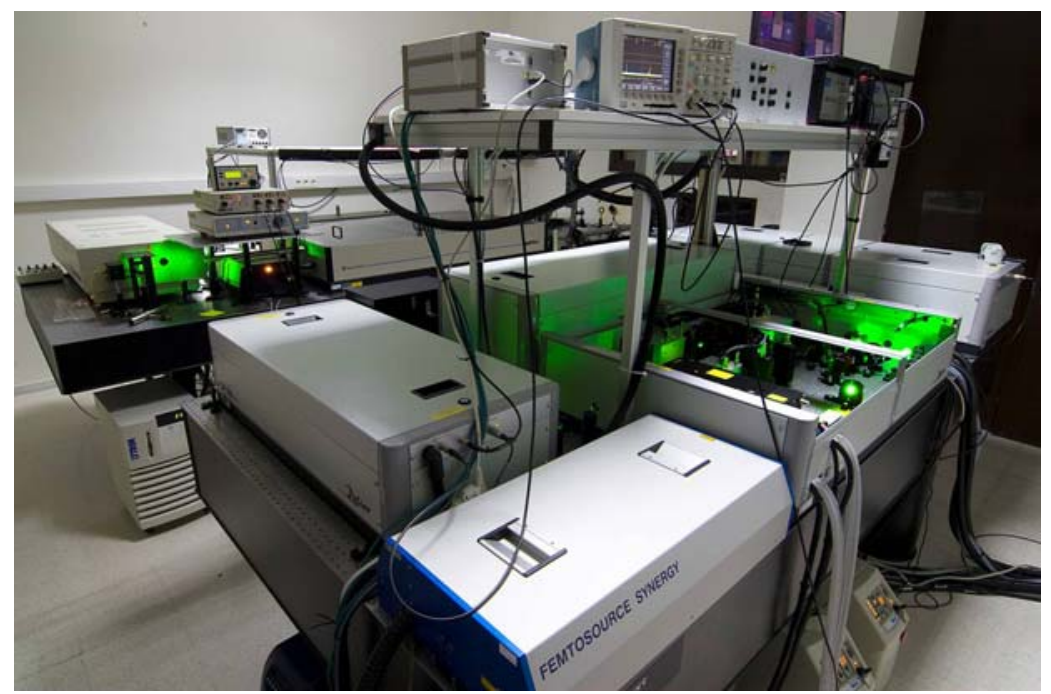

Figure 1.3. Photograph of the two CPA systems in the laboratory in Salamanca. At the front of the photo is the high-energy system (500 mJ/per pulse, Amplitude Technologies). In the back part of the photo is the Spectra-Physics system with two outputs: $1 \mathrm{~mJ}$ and $50 \mathrm{~mJ} / \mathrm{per}$ pulse.

\subsubsection{The OPCPA Technique}

However, CPA also has its limitations, and a new technique was proposed in 1992 [15] called Optical Parametric Chirped Pulse Amplification (OPCPA), which bears some similarities with the CPA but is based on a different process of amplification: a parametric nonlinear process termed Optical Parametric Amplification (OPA).

In the OPA process, the signal beam, the one to be amplified, propagates through the nonlinear crystal together with a pump beam of shorter wavelength. The photons of the pump wave are converted into signal photons and the same number of idler photons; the photon energy of the idler wave is the difference between the photon energies of the pump and signal waves. Since all the energy from the pump is transferred to the signal and idler beams, the crystal is not subjected to any significant thermal load, allowing high-power operation. This is one of the advantages of the OPCPA in comparison with conventional CPA.

Moreover, in a single pass the gain is huge and therefore the contrast of intensity between the amplified and non-amplified pulses is very high. This also means that it is not necessary to implement complicated multiple-pass set-ups to achieve a certain level of amplification.

Finally, parametric amplification is possible over a broad range of wavelengths, not defined by the crystal's gain bandwidth but for phase-matching conditions. Owing to these favourable properties, it offers an alternative amplification scheme, also reaching the PW level $[16,17]$. 
However, when passing to ultrahigh intensities OPCPA also has its limitations, and one of the solutions proposed is to build hybrid systems that combine OPCPA in the initial stages (front-end) and conventional laser amplification in the power amplifiers. In particular, this was the option in the Texas (U.S.A.) Petawatt with three OPCPA stages and four Nd:glass amplifiers reaching 1.1 PW peak power [18].

\subsection{Applications of Ultraintense Lasers}

With the above described advances, femtosecond lasers (not the very energetic ones) have gained in reliability and user-friendliness, and they have become "ready-to-use" systems available to a broad community of non-specialists.

The purpose of generating these short events can be understood within the context of the timeresolved imaging of physical processes. In such experiments, an event with a temporal duration shorter than the time constant of the process under study is required. As mentioned, many processes of physical, chemical or biological interest proceed on an ultrafast time scale. The best possible direct investigation of such rapid processes is a pump-probe experiment, where the sample of interest is disturbed with a first light pulse and subsequently the evolving state of the system is probed with a second pulse. By changing the relative delay between the arrivals of the two pulses we can obtain information about the different stages of the system. In such experiments, the temporal resolution is limited by the duration of the light pulses.

Thus, researchers are now interested in implementing the applications of these lasers as well as in the new developments in their technology [19]. Current efforts are focused, on the one hand, on obtaining more energetic pulses and, on the other, on reducing their temporal duration. The combination of both has resulted in the possibility of reaching intensities in the $10^{22} \mathrm{~W} / \mathrm{cm}^{2}$ range [20]. With these extremely high intensities it is possible to study new regimes of light-matter interactions, with "exotic" applications in laser fusion and particle acceleration.

In this context, each decrease in laser pulse duration provided shorter and shorter temporal events, with which it has become possible to time-resolve even faster events [21]. Nanosecond and picosecond pulses have enabled the imaging of plasma processes, while femtosecond pulses have enabled the dynamic imaging of molecular processes in chemistry. Also, the direct measurement of the electric field of a few-cycle laser pulse has been demonstrated, where the field dynamics has been resolved with attosecond (as, $10^{-15} \mathrm{~s}$ ) precision using a 250 as pulse [22]. The incredibly short duration of these attosecond pulses has opened a new regime for the measurement of electronic processes in atoms and molecules, allowing electron dynamics to be resolved for the first time. However, in these latter applications the laser intensity required to produce these short events is higher than the one used in this thesis [23].

\subsubsection{Secondary Sources}

The higher gain bandwidth of the Ti:Sapphire, in comparison with other crystals, offers the possibility of some wavelength tuning, although it is limited to the laser transitions allowable in the medium. For most spectroscopic experiments one does need, however, pulses at differing wavelengths, dictated by the absorption features of the sample, in order to excite a given transition. A convenient method for deriving tunable pulses from a regeneratively amplified system is optical parametric conversion, (OPA, for example). This will be another important part of the thesis, and also corresponds to another revolution in the field of ultrafast optics. Additionally, the availability 
of tunable lasers expands the number of applications of these systems as well as their potential users.

Originally, tunable femtosecond optical sources were based on dye lasers. In this case, some frequency tunability could be achieved simply by changing the laser dye. This flexibility, however, came at the expense of a complicated and time consuming re-optimization.

The increase in peak power with the introduction of the CPA technique made it possible to access a whole new class of nonlinear optical phenomena, triggering a renaissance in the field of nonlinear optics. Parallel to the developments in laser technology has been the discovery of novel nonlinear optical crystals such as $\mathrm{BBO}\left(\beta-\mathrm{BaB}_{2} \mathrm{O}_{4}\right)$ and $\mathrm{LBO}\left(\mathrm{LiB}_{3} \mathrm{O}_{5}\right)$, combining improved optical characteristics (high nonlinear optical coefficients, low group-velocity dispersion, and broad transparency ranges), with high damage thresholds. In particular, the BBO crystal is the nonlinear crystal used in the different optical set-ups reported in here.

\subsubsection{Application of Ultraintense Lasers to Materials Micro-Processing}

The field of materials micro-processing has also been revolutionised by developments in ultraintense laser technology. In fact, femtosecond lasers have become essential tools in this field. Some special properties of light-matter interactions appear only in the ultrashort pulse regime, and have led to the development of high-precision applications at the micro/nanometre scale that were not possible with systems of longer pulse duration (nanoseconds or picoseconds) [24].

Regarding the use of ultrashort pulses for materials micro-processing, very high peak powers are not required: a regeneratively amplified system suffices, although focusing the beam is mandatory in order to reduce the irradiated area and to achieve a high-resolution material structuring. For many practical applications it is also interesting that the system should have a high repetition rate $(\sim \mathrm{kHz})$ in order to reduce the processing time as much as possible.

In this short-pulse regime, only a very small fraction of the laser energy is transmitted as heat and transferred to the material surrounding the laser-irradiated area. The femtosecond pulses allow the local modification of certain physical properties of any type of material as well as its selective removal in a process known as ultrafast ablation, with minimal thermal or mechanical damage to the surrounding material. Processed samples of very high quality can be obtained in many kinds of materials, ranging from metals to transparent dielectrics.

An important issue is the study of the effect of the laser wavelength on the mechanism that leads to the modification of the material. Both the linear and nonlinear properties of laser-matter interactions at high intensities are strongly dependent on the laser wavelength. In particular, it is well known that the damage threshold (i.e. the minimum fluence needed to produce the modification/ablation of the material) decreases with wavelength. Therefore, it is of great interest to change the range of emission of femtosecond laser sources in order to perform more fundamental studies addressing this topic. Moreover, certain applications require the use of a specific laser wavelength in order to enhance or decrease the linear absorption in the material.

In this respect, at the laboratory of Micro-procesado de materiales con láser of Salamanca, in collaboration with other research groups in Spain, some projects have been started. In particular, the second-harmonic pulses of the Ti:Sapphire laser have been applied in the production of a patterning 
structure on the surface of silicon plates, in the manufacture of optical waveguides in active laser materials, and in the nano-structuring of thin film polymers, among many others.

\subsection{Thesis Summary}

The main objective of this thesis can be summarized as the study, development and application of techniques to change the range of emission of the femtosecond laser source available at the laboratory of Salamanca, applying the principles of frequency conversion in nonlinear crystals. Our aim was to increase the number of available auxiliary sources in the laboratory, with the intention of integrating these sources in the laboratory of Micro-procesado de materiales con láser so they could be applied in the study of light-matter interactions. Although the fundamentals of these processes are well known, and there are commercial systems to this end, they were not available in the lab and the optimum implementation for a specific laser beam required a detailed study for both the design and for the characterization of the beam outputs. Moreover, there was no previous experience on frequency-conversion techniques on our lab and thus, even the most fundamental experiments had to be done in order to get a reasonable "know-how".

The work is divided into three main parts. The first part is devoted to the generation of the second and third harmonics of the amplified Ti:Sapphire laser. We built an optical setup to produce $398 \mathrm{~nm}$ and $265 \mathrm{~nm}$ femtosecond pulses that could be transported easily from one experiment to another. These new UV laser sources are currently available in the laboratory of Micro-procesado de materiales con láser. In this dissertation, some of the applications in which they have been employed successfully are briefly described.

The second part deals with the improvement and control of certain properties of the beams generated with frequency mixing techniques. To this end, we used a combination of elements of diffractive optics with the above-mentioned frequency-conversion processes in nonlinear crystals. We followed two approaches. On the one hand, we introduced ultrafast micro-structuring techniques to integrate diffraction gratings on the surface of the nonlinear crystal in order to trigger noncollinear processes. On the other hand, diffractive lenses were used to control the spectra of the second and third harmonics of the laser. The fundamentals and applications of both techniques are described in this work.

Finally, we report our progress in OPA. This process, based on difference-frequency generation, allows the generation of continuously tunable wavelengths in certain spectral ranges. In particular, we focused our work on the development of an OPA system to produce tunable femtosecond pulses in the visible part of the spectrum. The seed pulses to be amplified are supercontinuum pulses generated in bulk materials. The design and implementation of the system are discussed. As a further step, we analyze in detail the properties of the supercontinuum generation stage, and some improvements to the standard set-ups are proposed. In particular we have introduced diffractive optics for the spectral control of such pulses, which in the future will be beneficial for the amplification process.

Below we offer a more detailed description of the different issues addressed in each chapter of the thesis.

Chapter 2: Introduction to Nonlinear Optics: Parametric Processes. In this chapter we revise the theoretical framework common to all the processes discussed in the thesis from the 
simple approach of monochromatic plane waves. However, in order to apply this theory to femtosecond pulses some modifications need to be made, even though certain observable facts are well represented in this simplified framework.

Chapter 3: Design, Characterization and Implementation of a Femtosecond SHG/THG Module. This chapter describes the steps that must be followed to obtain a homemade module that allows the doubling and tripling of the wavelength of the ultrafast laser source. Some of the applications of this module will be described in Chapter 8, which refers to materials microprocessing.

Chapter 4: Third Harmonic Generation in Micro-Structured BBO Crystals. In this chapter we describe the manufacture of a diffractive grating on the surface of a nonlinear crystal: BBO. The femtosecond laser was used for the micro-structuring of the crystal. Then, the customized photonic device was used as a mean of femtosecond pulse characterization and the multiplexing of second and third harmonic femtosecond pulses.

Chapter 5: Second and Third Harmonic Generation with Diffractive Lenses. In this chapter the same processes of second and third harmonic generation are studied but we introduce a diffractive optics element (a kinoform diffractive lens). This will help us to obtain wavelength tunability in the pulses thus generated, an effect that does not occur in common refractive focusing.

Chapter 6: Supercontinuum Generation in Bulk Media with Diffractive Lenses. In this part of the work we used the same diffractive lens, but on this occasion the nonlinear study process was supercontinuum generation in bulk media. With diffractive focusing, new features in the supercontinuum signal appeared, such as a longer spreading of the blue edge.

Chapter 7: Design and Implementation of a Noncollinear OPA in the Visible for fs Pulses. The noncollinear OPA described in this chapter was the first tunable source available in the laboratory of Salamanca, with a tunability that covers the visible range from $450-750 \mathrm{~nm}$. Moreover, we applied the OPA to the characterization of supercontinuum pulses generated in bulk media.

Chapter 8: Materials Micro-Processing with Vis-UV Femtosecond Pulses: Applications. In this chapter we present some of the experiments that have been carried out at the laboratory of Micro-procesado de materiales con láser of the Universidad de Salamanca concerning the microprocessing of materials with femtosecond pulses in the second and third harmonic of the femtosecond laser generated with the module described in Chapter 3.

\subsection{Publications}

The results presented along this thesis have been published in the following papers:

- C. Romero, J. R. Vázquez de Aldana, C. Mendez, and L. Roso, "Non-collinear sumfrequency generation of femtosecond pulses in a micro-structured $\beta-\mathrm{BaB}_{2} \mathrm{O}_{4}$ crystal," Optics Express 16, 18109-18117 (2008).

- G. Minguez-Vega, C. Romero, O. Mendoza-Yero, J. R. Vázquez de Aldana, R. BorregoVarillas, C. Mendez, P. Andres, J. Lancis, V. Climent, and L. Roso, "Wavelength tuning of 
femtosecond pulses generated in nonlinear crystals by using diffractive lenses," Optics Letters 35, 3694-3696 (2010).

- C. Romero, R. Borrego-Varillas, A. Camino, G. Minguez-Vega, O. Mendoza-Yero, J. Hernandez-Toro, and J. R. Vázquez de Aldana, "Diffractive optics for spectral control of the supercontinuum generated in sapphire with femtosecond pulses," Optics Express 19, 4977-4984 (2011).

- M. Oujja, A. Garcia, C. Romero, J. R. Vázquez de Aldana, P. Moreno, and M. Castillejo, "UV laser removal of varnish on tempera paints with nanosecond and femtosecond pulses," Physical Chemistry Chemical Physics 13, 4625-4631 (2011).

- C. Romero, R. Borrego-Varillas, O. Mendoza-Yero, G. Mínguez-Vega, C. Méndez, and J. R. Vázquez de Aldana, "Second-harmonic generation of femtosecond pulses focused on BBO with a diffractive lens." In preparation.

- R. Borrego-Varillas, C. Romero, O. Mendoza-Yero, G. Mínguez-Vega, and J. R. Vázquez de Aldana "Supercontinuum visible femtosecond pulses generated in sapphire with diffractive lenses." In preparation.

Other results not discussed in the thesis have been published in:

- R. Borrego-Varillas, C. Romero, J. R. Vázquez de Aldana, J. M. Bueno, and L. Roso, "Wavefront retrieval of amplified femtosecond beams by second-harmonic generation," Optics Express 19, 22851-22862 (2011).

- N. N. Dong, Y. Tan, A. Benayas, J. Vázquez de Aldana, D. Jaque, C. Romero, F. Chen, and Q. M. Lu, "Femtosecond laser writing of multifunctional optical waveguides in a Nd:YVO(4)+KTP hybrid system," Optics Letters 36, 975-977 (2011).

- G. Raj Kumar, J. J. Carvajal, M. C. Pujol, X. Mateos, M. Aguiló, F. Diaz, J. R. Vázquez de Aldana, C. Romero, C. Méndez, P. Moreno and L. Roso, "Fabrication and Characterization of Photonic Structures in Crystals of the $\mathrm{KTiOPO}_{4}$ Family", Physics Procedia 8, 126-135 (2010).

- G. Raj Kumar, J. J. Carvajal, M. C. Pujol, X. Mateos, M. Aguiló, F. Diaz, J. R. Vázquez de Aldana, C. Romero, C. Méndez, P. Moreno, L. Roso, J. Ferré-Borrull, J. Pallarès, L. F. Marsal, R. Macovez, and J. Martorell, "Analysis of Linear and Nonlinear Optical Properties of Diffraction Gratings Inscribed on the Surface of Single Crystals of the $\mathrm{KTiOPO}_{4}$ Family", Proceedings of SPIE 7728, 772817 (2010). 



\section{Chapter 2}

\section{Introduction to Nonlinear Optics: Parametric Processes}

This chapter serves to give a common framework to all the nonlinear processes that will be addressed along this thesis, all of them dealing with the generation of new frequency components of the electromagnetic field. In our experiments, we made use of one, two, or even three of these processes at the same time. Thus, we have considered it of importance to give a general idea about them under the very simple treatment of monochromatic plane waves interacting with matter. Despite this, femtosecond pulses cannot be treated as monochromatic waves, and some new considerations will be needed to reproduce the experimental observations more accurately. Nevertheless, some of the effects that will be seen in the femtosecond regime can be understood under the premises of this simple basis.

\subsection{Introduction to Nonlinear Optics}

Taking a definition from reference [25], Nonlinear Optics is the study of phenomena that occur as a consequence of the modification in the optical properties of a material system by the presence of light. Typically, only laser light can be sufficiently intense to modify the optical properties of a material system. If the light interacting with the matter is intense enough, all the materials can, in principle, exhibit a nonlinear response to an applied electric field; in other words, any material system can be nonlinear. In our opinion, the air is the most surprising example. It might be expected that it would be rather difficult to observe nonlinear effects in this medium, because in classical linear optics it is quite common to attribute the same properties to air as for a vacuum. However, if we propagate an ultraintense laser beam in air many nonlinear effects appear [26], self-focusing 
being only one of them. The peak power (for a nearly Gaussian beam) necessary to observe these effects is around $3 \mathrm{GW}$. This may sound extremely high, but currently the limits in laser power exceed this value considerably, even reaching the PW level, as mentioned in the previous chapter. However, there are materials that possess certain characteristics that make them adequate for observing nonlinear effects, even with moderate intensities. We shall refer to them as nonlinear materials.

Nonlinear optical phenomena are nonlinear in the sense that they occur when the response of the material system to an applied optical field depends on the strength of the electric field itself in a nonlinear manner. Let us take the Second-Harmonic Generation (SHG) process as an example; as we shall see later, SHG depends on the part of the atomic response that scales quadratically with the optical field applied [25, 27]. As a consequence of this, the intensity of the light generated at the second-harmonic frequency tends to increase with the square of the intensity of the applied laser light.

For a better understanding of what an optical nonlinearity is, we need to know how the dipole moment per unit volume, or the polarization ${ }^{2} \tilde{P}(t)$ of a material system, depends on the strength of an applied optical field $\tilde{E}(t)$. In the conventional case, i.e., linear optics, the polarization induced in the medium depends linearly on the electric field and can be described by the following relation:

$$
\tilde{P}(t)=\varepsilon_{0} \chi^{(1)} \tilde{E}(t)
$$

where the proportionality constant $\chi^{(1)}$ is the well known linear susceptibility, and $\varepsilon_{0}$ is the vacuum permittivity. However, in nonlinear optics, the optical response can be expressed in a generalized way, expanding the polarization $\tilde{P}(t)$ in a power series of the electric field strength [28]:

$$
\begin{aligned}
\tilde{P} & =\varepsilon_{0}\left[\chi^{(1)} \tilde{E}(t)+\chi^{(2)} \tilde{E}^{2}(t)+\chi^{(3)} \tilde{E}^{3}(t)+\ldots\right] \\
& \equiv \tilde{P}^{(1)}(t)+\tilde{P}^{(2)}(t)+\tilde{P}^{(3)}(t)+\ldots
\end{aligned}
$$

where $\chi^{(2)}$ and $\chi^{(3)}$ are respectively the second- and third-order nonlinear optical susceptibilities, and so on. For simplicity, the polarization and the electric field are treated as scalar quantities. The susceptibilities will become tensors when this equation is generalized to treat the vector nature of the fields. In these equations, the assumption is made that the medium responds instantaneously to the applied electric field. This means that the polarization at time $t$ depends only on the instantaneous value of the electric field strength. The latter also implies, through the KramersKroning relations, that the medium is dispersionless and lossless ${ }^{3}$. In general the nonlinear susceptibilities depend on the frequency of the applied fields, but under the assumption of instantaneous response we can take them as constants. In this simple approach, we can refer to the following quantities:

$$
\begin{aligned}
& \tilde{P}^{(2)}(t)=\varepsilon_{0} \chi^{(2)} \tilde{E}^{2}(t) \\
& \tilde{P}^{(3)}(t)=\varepsilon_{0} \chi^{(3)} \tilde{E}^{3}(t)
\end{aligned}
$$

\footnotetext{
${ }^{2}$ The tilde $(\sim)$ denotes a quantity that varies rapidly with time. This is useful for distinguishing the slowly varying amplitudes from the total electric field oscillating at frequency $\omega$.

${ }^{3}$ See Appendix A for a generalization of these equations to the case of a medium with dispersion and loss and a discussion of the Kramers-Kroning relations.
} 
as the second- and third-order nonlinear polarizations, respectively. The physical processes that may occur as a result of the second-order polarization are different from those that occur as result of the third-order polarization. Moreover, the nonlinear interactions of second-order only occur in non-centrosymmetric media; i.e., media without inversion symmetry. Liquids, gases and amorphous solids, such as glass, have inversion symmetry, and the second-order susceptibility $\chi^{(2)}$ equals zero in this kind of medium. As a result, these material systems cannot produce optical nonlinearities of second order. In contrast, the third-order nonlinear optical interactions, i.e., those described by $\chi^{(3)}$, can occur in both centrosymmetric and non-centrosymmetric media. In the next section we shall discuss the physical origin of this behaviour.

These nonlinear polarization terms become large enough to produce observable effects only for fields comparable in their amplitude with the interatomic field acting upon the electron. This is the reason why it was only after the advent of the laser, as a new and powerful light source, that nonlinear optics became amenable to experimental study. At this point it would be interesting to make a rough estimation of the order of magnitude of these second- and third-order nonlinear susceptibilities. One would expect that the correction factor of the lowest order, $\tilde{P}^{(2)}$, in Eq. (2.2) would be comparable, or at least would be of the same order of magnitude, to the linear response $\tilde{P}^{(1)}$. In contrast, if $\tilde{P}^{(2)}$ were much lower the nonlinear effects could not be observed. If the amplitude of the applie d electric field $E$ is of the same order of magnitude as the characteristic atomic electric field, $E_{a t}$, and considering, $\tilde{P}^{(1)}(t) \approx \tilde{P}^{(2)}(t)$ we expect that the second-order susceptibility $\chi^{(2)}$ might be of the order of $\chi^{(1)} / E_{a t}$. The value of the interatomic electric field can be calculated and is equal to $E_{a t}=5.14 \times 10^{11} \mathrm{~V} / \mathrm{m}$. In the case of condensed matter $\chi^{(1)}$ is of the order of unity ${ }^{4}$, and therefore we expect that $\chi^{(2)}$ is simply the inverse of the atomic electric field strength:

$$
\chi^{(2)} \simeq 1.94 \times 10^{-12} \mathrm{~m} / \mathrm{V}
$$

In a similar way $\chi^{(3)}$ in condensed matter might be equal to $\chi^{(1)} / E_{a t}^{2}$

$$
\chi^{(3)} \simeq 3.78 \times 10^{-24} \mathrm{~m}^{2} / \mathrm{V}^{2}
$$

In fact, this calculation -although very simple- fits in well with the measured values of $\chi^{(2)}$ and $\chi^{(3)}$, which can be consulted in text books: for example, in reference [25], Tables 1.5.3 and Tables 4.3.1 respectively.

It is important to recall that in this simple approach we are making use of a perturbative description of the light-matter interaction Eq. (2.2). This works well when the intensities of the applied electric fields are "moderate". However, when the intensities are much higher, phenomena such as photo-ionization or the generation of high-order harmonics take place, and these phenomena cannot be explained on the basis of this simplified theory. To study the physics underlying such phenomena, new models (non-pertubative) must be applied. It is possible to calculate an upper limit to the validity of the following theory as the corresponding intensity value associated with an applied field equal to the interatomic field:

\footnotetext{
${ }^{4}$ This can be justified in terms of the linear index of refraction, which is defined as: $n^{2}=1+\chi^{(1)} ; n$ is of the order of 1.5 for condensed matter and hence $\chi^{(1)} \approx 1$.
} 


$$
I_{a t}=\frac{1}{2} \varepsilon_{0} c E_{a t}^{2}=3.5 \times 10^{16} \mathrm{~W} / \mathrm{cm}^{2}
$$

There are laser systems able to produce these ultraintense laser fields, and in fact the current record in peak intensity $\left(\sim 10^{22} \mathrm{~W} / \mathrm{cm}^{2}\right)$ exceeds this value by six orders of magnitude [20]. Strongfield nonlinear optics deals with the processes arising when matter interacts with such ultraintense lasers.

\subsection{Microscopic Classical Model of the Light-Matter Interaction}

There are different possibilities of addressing the problem of describing the light-matter interaction. Light can be considered either quantum or classical, as well as matter. A quantum model will treat both light and matter as quantum systems; a semi-classical model will preserve the quantum characteristics of matter, but light would be classical. This is the framework of the strong-field nonlinear optics mentioned above. Finally, in a purely classical model light and matter would be classical. We shall choose the latter model to describe the nonlinear processes in which we are interested; only some references to the quantum nature of matter will be made at some point. Recall that this perturbative approach, although classical, works well for describing the processes in which we are interested.

Upon describing conventional classical linear optics, the Lorentz model of the atom considers that the electron is linked to the nucleus under the influence of a harmonic oscillator potential. This is a good approximation to describe the interaction of atoms with a non-intense electric field. By solving the equation of movement for the electron in this potential [29], the microscopic nature of the linear susceptibility is obtained. At this point, it is useful to note that the dipole moment per unit volume equals:

$$
\mathbf{P}=-N e \mathbf{x}
$$

where $N$ is the density of charges; $-e$ is the electron's charge, and $\mathbf{x}$ is the displacement of the electric dipole. We have also introduced the linear polarization in Eq. (2.1), where $\chi^{(1)}$ was the proportionality constant. Therefore, solving the equation of motion in one dimension ${ }^{5}$, and considering a response after a fairly long time, one obtains a value for $x(\mathrm{t})$. Then, by making use of Eq. (2.8) and the relationship in (2.1), the linear susceptibility is obtained in terms of the parameters of the incoming field, $\omega$, and material properties such as the frequency of resonance, $\omega_{0}$ and the damping force coefficient, $\gamma$ :

$$
\chi^{(1)}=\frac{N\left(e^{2} / m\right)}{\varepsilon_{0}\left(\omega_{0}^{2}-\omega^{2}-2 i \omega \gamma\right)}
$$

However, to account for second- and third-order susceptibilities, we shall allow the electrons to be linked to the atom through a more general potential, or analogously, by allowing the possibility of a nonlinearity in the restoring force of the oscillator [25]. We shall explain how the details of the analysis differ depending upon whether the medium has inversion symmetry or not. In other words,

\footnotetext{
${ }^{5}$ The equation in 1D is as follows: $\ddot{\tilde{x}}+2 \gamma \dot{\tilde{x}}+\omega_{0}^{2} \tilde{x}=-e \tilde{E}(t) / m$, where $\tilde{E}(t)=E e^{-i \omega t}$. For simplicity, we use a scalar-field approximation, and hence we can consider only $1 \mathrm{D}$ in the motion equation.
} 
in linear optics the field strength of the incident light is low enough for the displacement of the electrons to be small, and the atomic potential can be well approximated by a parabolic potential or a linear restoring force. The linear interactions are well described by this model, an example being the physical origin of the refraction index.

In order to continue with the analysis to include the nonlinear terms, materials are split into two groups, depending on the symmetry of the potential at which the electrons are bound: centrosymmetric and non-centrosymmetric media. Figure 2.1 shows some examples of the associated energy potentials for the two cases -red lines-. The parabola associated with the harmonic oscillator potential is also shown for comparison -blue lines-.

a)

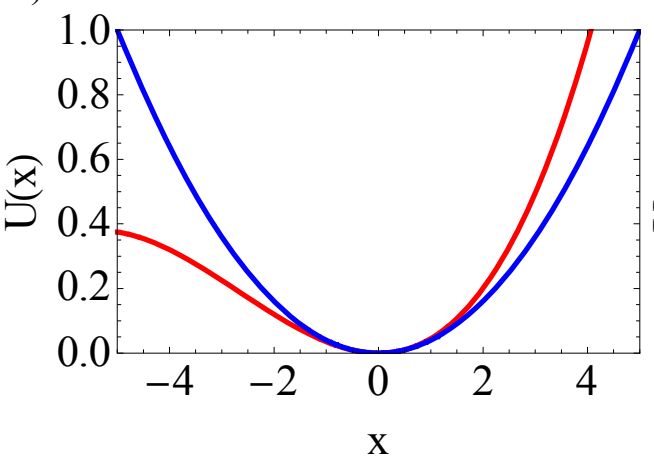

b)

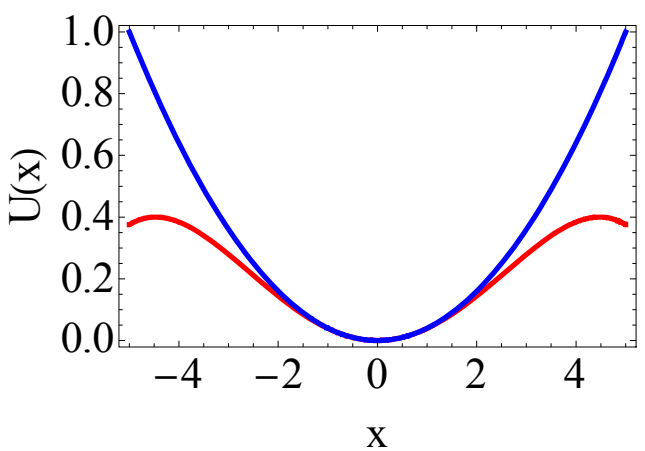

Figure 2.1. Potential energy functions for a non-centrosymmetric medium-red line in panel a)- and for a centrosymmetric medium -red line in panel b)-. In both cases, the harmonic potential-blue line-is included for comparison.

With a view to simplify the study, we shall consider that the electron is close to a potential minimum; otherwise, more complicated effects such as ionization should be considered. Therefore, since the electron is in this situation close to equilibrium, we are allowed to make a Taylor expansion of these potentials around the minimum for the above-mentioned cases.

$$
U(x) \cong U_{0}+\left.\frac{d U}{d x}\right|_{x=0} x+\left.\frac{1}{2} \frac{d^{2} U}{d x^{2}}\right|_{x=0} x^{2}+\left.\frac{1}{3 !} \frac{d^{3} U}{d x^{3}}\right|_{x=0} x^{3}+\left.\frac{1}{4 !} \frac{d^{4} U}{d x^{4}}\right|_{x=0} x^{4}
$$

The first term in the expansion is a constant, which only determines the potential energy and can therefore be neglected. The next term is zero because we are assuming that the electron is in a potential minimum. Finally, the third term can easily be recognized as the harmonic potential (because of the quadratic dependence on the displacement, $x$ ). In the case of a non-centrosymmetric material, we shall consider this potential only up to the next order: the third-order term. Thus, the approximated potential is represented in Eq. (2.11), $a$ being a constant that gives an idea of the strength of the nonlinearity and where the electron's mass has been included in the equation for future convenience.

$$
U(x) \cong \frac{1}{2} m \omega_{0}^{2} \tilde{x}^{2}+\frac{1}{3} m a \tilde{x}^{3}
$$


Note that this potential is non-symmetric under the $x \rightarrow-x$ transformation. In fact it does not have a defined symmetry: $U(x) \neq U(-x) \neq-U(-x)$. Let us see how the first derivative will lead us to the following restoring force:

$$
F_{\text {restoring }}=-\frac{d U(x)}{d x}=-m \omega_{0}^{2} \tilde{x}-m a \tilde{x}^{2}
$$

which is nonlinear with displacement $x$, as was already mentioned in the beginning of this section. Thus, the equation that describes the movement of the electron in the previous potential under the influence of an applied electric field $\tilde{E}(t)$ is:

$$
\ddot{\tilde{x}}+2 \gamma \dot{\tilde{x}}+\omega_{0}^{2} \tilde{x}+a \tilde{x}^{2}=-e \tilde{E}(t) / m
$$

where $-e$ is the charge of the electron and the term $-2 m \gamma \dot{x}$ corresponds to the damping force. The origin of this force comes from the spontaneous emission of radiation if we consider a single atom interacting with its neighbours in a solid. We may consider that the applied electric field has the form $^{6}$ :

$$
\tilde{E}(t)=E_{1} e^{-i \omega_{1} t}+E_{2} e^{-i \omega_{2} t}+c c
$$

Owing to the nonlinear term, Eq. (2.13) has no exact analytical solution. However, if the applied electric field is not too large, the nonlinear term $a \tilde{x}^{2}$ will be much smaller than the linear $\omega_{0}^{2} \tilde{x}$ term for any displacement introduced by the applied electric field. Under this circumstance, we can solve Eq. (2.13) by a perturbation expansion. Following the resolution in, for example, reference [25] or any other text book, one finds that there are different responses in the medium that oscillate with the frequencies involved in the square of the incoming electric field, $\tilde{E}(t)^{2}$. In particular, these responses oscillate with frequencies of $\pm 2 \omega_{1}, \pm 2 \omega_{2}, \pm\left(\omega_{1}+\omega_{2}\right), \pm\left(\omega_{1}-\omega_{2}\right)$.

$$
\begin{aligned}
\tilde{x}(t) & =\sum_{i=1}^{2} x^{(1)}\left(\omega_{i}\right) e^{-i \omega_{i} t}+\sum_{i=1}^{2} x^{(2)}\left(2 \omega_{i}\right) e^{-i 2 \omega_{i} t}+x^{(2)}\left(\omega_{1}+\omega_{2}\right) e^{-i\left(\omega_{1}+\omega_{2}\right) t} \\
& +x^{(2)}\left(\omega_{1}-\omega_{2}\right) e^{-i\left(\omega_{1}-\omega_{2}\right) t}+x^{(2)}(0)+c c
\end{aligned}
$$

Accordingly, applying the relationship $\tilde{P}(t)=-N e \tilde{x}(t)$ in a similar way, as is done in the case of the linear interaction, we can see how the polarization is no longer proportional to the incoming electric field.

Regarding the other kind of medium that we have already introduced, centrosymmetric, the potential would be:

$$
U(\tilde{x})=\frac{1}{2} m \omega_{0}^{2} \tilde{x}^{2}-\frac{1}{4} m b \tilde{x}^{4}
$$

\footnotetext{
${ }^{6}$ In media described by the second-order nonlinear susceptibility it is common to have two monochromatic waves of different frequencies entering the medium, and in media where the dominant one is the third-order susceptibility it is usual to have three waves.
} 
In this case we must cancel the third-order term, because, as we have said, the potential must have inversion symmetry. Under the $x \rightarrow-x$ transformation, one obtains $U(x)=U(-x)$. This has an important implication, because these media do not have second-order susceptibility. Thus, processes such as SHG, which we have alluded to previously and which will be described in the next section, cannot take place in this kind of media. However, the other way is possible; in media with second-order susceptibility the third-order susceptibility can also be non-zero. In this model, this fact can be demonstrated simply by taking the next term in the expansion of the potential: Eq. (2.11).

\subsection{The Wave Equation for Nonlinear Optical Media}

Up to here, we have seen how the nonlinear response of a material system to an intense laser field can cause the polarization of the medium to develop new frequency components not present in the incident radiation field. These new frequency components of the polarization act as sources of new frequency components of the electromagnetic field. Accordingly, the next logical step is to analyze how Maxwell's equations describe the generation of these new components of the field and, more generally, we shall see how the various frequency components of the field become coupled by the nonlinear interaction. Thus, beginning with Maxwell's equations, in the SI [30]:

$$
\begin{gathered}
\nabla \cdot \tilde{\mathbf{D}}=\tilde{\rho} \\
\nabla \cdot \tilde{\mathbf{B}}=0 \\
\nabla \times \tilde{\mathbf{E}}=-\frac{\partial \tilde{\mathbf{B}}}{\partial t} \\
\nabla \times \tilde{\mathbf{H}}=\frac{\partial \tilde{\mathbf{D}}}{\partial t}+\tilde{\mathbf{J}}
\end{gathered}
$$

We are interested in the solution of these equations in regions where there are neither free charges nor free currents; in principle we shall study the interaction with non-ionized media.

$$
\tilde{\rho}=0, \quad \tilde{\mathbf{J}}=0
$$

We also assume that the material is not magnetic; thus:

$$
\tilde{\mathbf{B}}=\mu_{0} \tilde{\mathbf{H}}
$$

However, we allow the material to be nonlinear, and this is what makes the difference with the normal treatment. The fields $\tilde{\mathbf{D}}$ and $\tilde{\mathbf{E}}$ are related by:

$$
\tilde{\mathbf{D}}=\varepsilon_{0} \tilde{\mathbf{E}}+\tilde{\mathbf{P}}
$$

From what was discussed in the previous section, the polarization vector $\tilde{\mathbf{P}}$ depends nonlinearly upon the local value of the electric field strength $\tilde{\mathbf{E}}$.

We can proceed to derive the optical wave equation in the usual manner, taking the curl in Eq. (2.19), then interchanging the order of the space and time derivatives on the right hand side of the resulting equation, and using Eqs. (2.20), (2.21) and (2.22) to replace $\nabla \times \tilde{\mathbf{B}}$ by $\mu_{0}(\partial \tilde{\mathbf{D}} / \partial t)$ we obtain: 


$$
\nabla \times \nabla \times \tilde{\mathbf{E}}+\mu_{0} \frac{\partial^{2} \tilde{\mathbf{D}}}{\partial t^{2}}=0
$$

The next step is to use Eq. (2.23) to eliminate $\tilde{\mathbf{D}}$ from the latter equation and we thus obtain:

$$
\nabla \times \nabla \times \tilde{\mathbf{E}}+\frac{1}{c^{2}} \frac{\partial^{2} \tilde{\mathbf{E}}}{\partial t^{2}}=-\frac{1}{\varepsilon_{0} c^{2}} \frac{\partial^{2} \tilde{\mathbf{P}}}{\partial t^{2}}
$$

where the expression $\varepsilon_{0} \mu_{0}=1 / c^{2}$ has been used to eliminate $\mu_{0}$ for future convenience. This is the most general form of the wave equation in nonlinear optics. Although it is a somewhat complicated equation; we can apply the following identity to try to simplify it:

$$
\nabla \times \nabla \times \tilde{\mathbf{E}}=\nabla(\nabla \cdot \tilde{\mathbf{E}})-\nabla^{2} \tilde{\mathbf{E}}
$$

In conventional linear optics the first term in Eq. (2.26) vanishes for the case of isotropic source free media, since Maxwell equation (2.17) implies $\nabla \cdot \tilde{\mathbf{E}}=0$. However, in nonlinear optics, the more general form of the relationship between $\tilde{\mathbf{D}}$ and $\tilde{\mathbf{E}}$ Eq. (2.23), does not allow us to cancel this term owing to Maxwell's equation (2.17), even when the medium is isotropic and source-free. However, this term can be dropped for some cases of interest: for example if we consider the case of transverse, infinite plane waves. Moreover, note that, for the case of anisotropic media (crystals) we must consider also that the angle between $\tilde{\mathbf{D}}$ and $\tilde{\mathbf{E}}$, the walk-off angle, is small. A detailed justification of this can be consulted in [31].

Finally, the wave equation can be taken to have the form:

$$
\nabla^{2} \tilde{\mathbf{E}}-\frac{1}{c^{2}} \frac{\partial^{2} \tilde{\mathbf{E}}}{\partial t^{2}}=\frac{1}{\varepsilon_{0} c^{2}} \frac{\partial^{2} \tilde{\mathbf{P}}}{\partial t^{2}}
$$

It is often convenient to split $\tilde{\mathbf{P}}$ into its linear and nonlinear parts:

$$
\tilde{\mathbf{P}}=\tilde{\mathbf{P}}^{(1)}+\tilde{\mathbf{P}}^{(N L)}
$$

We can identify $\tilde{\mathbf{P}}^{(1)}$ as the part of the polarization that depends linearly on the electric field strength $\tilde{\mathbf{E}}$. We can introduce this in the definition of the electric displacement field, Eq.(2.23):

$$
\tilde{\mathbf{D}}=\varepsilon_{0} \tilde{\mathbf{E}}+\tilde{\mathbf{P}}^{(1)}+\tilde{\mathbf{P}}^{(N L)}
$$

Considering $\tilde{\mathbf{D}}^{(1)}$ as the linear part in the electric displacement, we have:

$$
\tilde{\mathbf{D}}^{(1)}=\varepsilon_{0} \tilde{\mathbf{E}}+\tilde{\mathbf{P}}^{(1)}
$$

In terms of this quantity, the wave equation (2.27) can be written as:

$$
\nabla^{2} \tilde{\mathbf{E}}-\frac{1}{\varepsilon_{0} c^{2}} \frac{\partial^{2} \tilde{\mathbf{D}}^{(1)}}{\partial t^{2}}=\frac{1}{\varepsilon_{0} c^{2}} \frac{\partial^{2} \tilde{\mathbf{P}}^{N L}}{\partial t^{2}}
$$

In the case of a lossless, dispersionless medium, we can express the relationship between $\tilde{\mathbf{D}}$ and $\tilde{\mathbf{E}}$ in terms of a real, frequency-independent dielectric tensor $\boldsymbol{\varepsilon}^{(1)}$ : 


$$
\tilde{\mathbf{D}}^{(1)}=\varepsilon_{0} \boldsymbol{\varepsilon}^{(1)} \cdot \tilde{\mathbf{E}}
$$

In the case of an isotropic material, the dielectric tensor becomes a scalar quantity and the previous relation reduces to simply:

$$
\tilde{\mathbf{D}}^{(1)}=\varepsilon_{0} \varepsilon^{(1)} \tilde{\mathbf{E}}
$$

where $\varepsilon_{0}=8.85 \times 10^{-12} \mathrm{~F} / \mathrm{m}$ is a fundamental constant: the permittivity of free space, whereas $\varepsilon^{(1)}$ is the dimensionless relative permittivity, which is different for each material. For the case of an isotropic, dispersionless material, the wave equation (2.31) becomes:

$$
\nabla^{2} \tilde{\mathbf{E}}-\frac{\varepsilon^{(1)}}{c^{2}} \frac{\partial^{2} \tilde{\mathbf{E}}}{\partial t^{2}}=\frac{1}{\varepsilon_{0} c^{2}} \frac{\partial^{2} \tilde{\mathbf{P}}^{N L}}{\partial t^{2}}
$$

This equation has the form of a driven (i.e. inhomogeneous) wave equation; the nonlinear response of the medium acts as a source term that appears on the right hand side of the equation. In the absence of this source term, this equation admits solutions of the form of free waves propagating with velocity $c / n$, where $n$ is the linear index of refraction defined as $n^{2}=\varepsilon^{(1)}$, and $c$ is the speed of light in vacuum. The term $\partial^{2} \tilde{P}^{N L} / \partial t^{2}$ is a measure of the acceleration of the charges that constitute the medium. Therefore, this equation is entirely consistent with Larmor's theorem of electromagnetism, which states that accelerated charges generate electromagnetic radiation.

When the medium is dispersive, we must consider a wave equation for each frequency component of the field:

$$
\nabla^{2} \tilde{\mathbf{E}}_{n}-\frac{\varepsilon^{(1)}\left(\omega_{n}\right)}{c^{2}} \frac{\partial^{2} \tilde{\mathbf{E}}_{n}}{\partial t^{2}}=\frac{1}{\varepsilon_{0} c^{2}} \frac{\partial^{2} \tilde{\mathbf{P}}_{n}^{N L}}{\partial t^{2}}
$$

\subsection{Description of Nonlinear Optical Processes}

The usual way of describing nonlinear optical processes is to express the time-dependent polarization in terms of the amplitude of the applied electric field, in the same way as done in Eq. (2.2). The reason why polarization plays an important role in the description of nonlinear optical phenomena is because the time-varying polarization acts as a source of new components of the electromagnetic field.

In this section we offer a brief qualitative description of a number of nonlinear optical processes that occur as a consequence of an induced nonlinear polarization [25, 27].

\subsubsection{The SHG Process}

The process of SHG [3]mentioned above is illustrated schematically in Figure 2.2. A plane wave, with its electric field strength given by

$$
\tilde{E}(t)=E e^{-i \omega t}+c c,
$$


enters a crystal with non-zero second-order nonlinear susceptibility. The nonlinear polarization created in the medium is given by expression (2.3); explicitly as:

$$
\tilde{P}^{(2)}(t)=2 \varepsilon_{0} \chi^{(2)} E E^{*}+\left(\varepsilon_{0} \chi^{(2)} E^{2} e^{-i 2 \omega t}+c c\right)
$$

At this point we observe how the second-order polarization consists of one zero-frequency contribution -the first term- plus a contribution at frequency $2 \omega-$ the second term-. According to the driven wave equation introduced previously, Eq. (2.34), this latter contribution can lead to the generation of radiation at the second-harmonic frequency, $2 \omega$.

The first contribution does not give rise to electromagnetic radiation because its second derivative vanishes. This term corresponds to a process called optical rectification, in which a static electric field is created along the nonlinear crystal.

a)

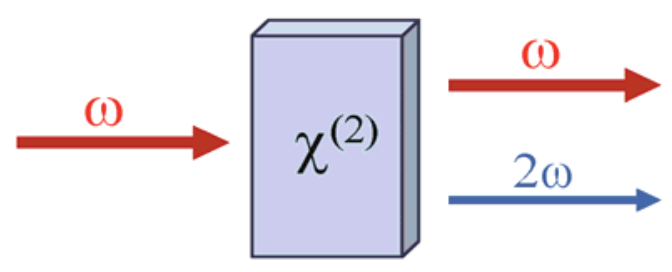

b)

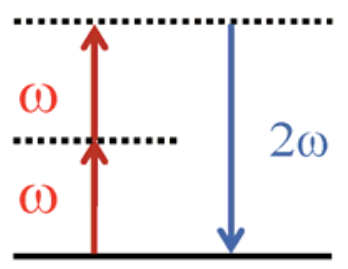

Figure 2.2. a) Representation of the SHG process. The wave at frequency $\omega$ enters a medium with second-order susceptibility and radiation at the doubled frequency is generated. b) Energy-level diagram of the same process. Dotted lines represent virtual states.

The generation of the second harmonic is a process normally used to obtain new frequencies from common laser systems such as Nd:YAG or Ti:Sapphire lasers, with emission in the infrared. By means of this process we can obtain frequencies in the visible spectrum $(532 \mathrm{~nm}$ and $400 \mathrm{~nm}$, respectively).

The SHG process can be also visualized in terms of photons interchanging between the different frequency components of the electric field, see Figure $2.2 \mathrm{~b}$ ). The latter is explained in the following way: two photons with frequency $\omega$ are destroyed and one photon with frequency $2 \omega$ is simultaneously created in a single quantum-mechanical process. The solid line in the figure represents the atomic ground state; the dashed lines represent what are known as virtual levels. It should be noted that these levels are not energy eigenlevels of the free atom, but rather represent the combined energy of one of the energy eigenstates of the atom and of one or more photons of the radiation field.

\subsubsection{Sum and Difference-Frequency Generation}

More generally, we can consider two monochromatic waves with different frequencies inciding on the nonlinear medium with second-order susceptibility. The incoming electric field is represented in the form:

$$
\tilde{E}(t)=E_{1} e^{-i \omega_{1} t}+E_{2} e^{-\omega_{2} t}+c c
$$


Again, if we take into account the fact that the second-order polarization is in the form of Eq. (2.3), the nonlinear polarization will be given by:

$$
\begin{aligned}
\tilde{P}^{(2)}(t)=\varepsilon_{0} \chi^{(2)}\left[E_{1}^{2} e^{-i 2 \omega_{1} t}\right. & \left.+E_{2}^{2} e^{-i 2 \omega_{2} t}+2 E_{1} E_{2} e^{-i\left(\omega_{1}+\omega_{2}\right) t}+2 E_{1} E_{2}^{*} e^{-i\left(\omega_{1}-\omega_{2}\right) t}+c c\right] \\
& +2 \varepsilon_{0} \chi^{(2)}\left[E_{1} E_{1}^{*}+E_{2} E_{2}^{*}\right]
\end{aligned}
$$

It is convenient to express the latter relationship in the form:

$$
\tilde{P}^{(2)}(t)=\sum_{n} P\left(\omega_{n}\right) e^{-i \omega_{n} t}
$$

where the summation extends over positive and negative frequencies, $\omega_{n}$. Therefore, from Eq. (2.39) the following complex amplitudes of the nonlinear polarization arise, one for each frequency component.

$$
\begin{aligned}
& P\left(2 \omega_{1}\right)=\varepsilon_{0} \chi^{(2)} E_{1}^{2} \quad(\mathrm{SHG}) \\
& P\left(2 \omega_{2}\right)=\varepsilon_{0} \chi^{(2)} E_{2}^{2} \quad(\mathrm{SHG}) \\
& P\left(\omega_{1}+\omega_{2}\right)=2 \varepsilon_{0} \chi^{(2)} E_{1} E_{2} \quad(\mathrm{SFG}) \\
& P\left(\omega_{1}-\omega_{2}\right)=2 \varepsilon_{0} \chi^{(2)} E_{1} E_{2}^{*} \quad(\mathrm{DFG}) \\
& P(0)=2 \varepsilon_{0} \chi^{(2)}\left(E_{1} E_{1}^{*}+E_{2} E_{2}^{*}\right) \quad(\mathrm{OR})
\end{aligned}
$$

Where the label on the right side represents the physical process that is described by each polarization: second-harmonic generation (SHG), Sum-Frequency Generation (SFG), DifferenceFrequency Generation (DFG) and optical rectification (OR).

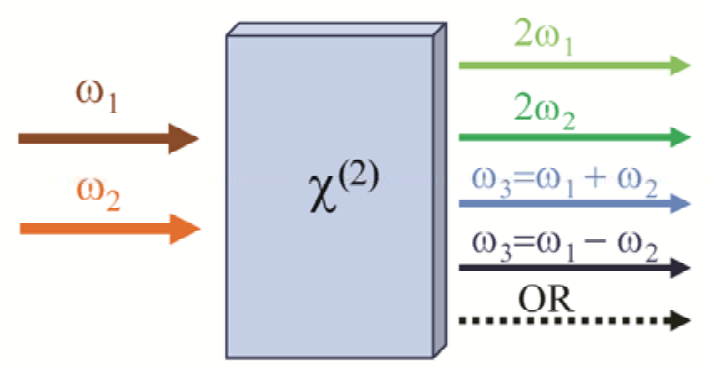

Figure 2.3. Representation of all the frequency components that can arise when two monochromatic electromagnetic waves, with different frequencies, enter a medium with second-order susceptibility.

From Eq. (2.41), we can see that four different nonzero frequency components are present in the nonlinear polarization. However, typically no more than one of these frequency components will be present with any appreciable intensity in the radiation generated by the nonlinear optical interaction. The reason for this behaviour is that nonlinear polarization can produce the signal only if certain phase-matching conditions are met at the same time. What usually happens is that the phase-matching conditions cannot be satisfied for the four frequency components at the same time. We shall discuss this latter below, but normally one chooses the frequency of the emitted radiation, choosing the polarization of the incident radiation and the orientation of the nonlinear crystal.

At this point, let us take a closer look into the processes of sum- and difference-frequency generation. In many ways, the process of SFG is analogous to that of SHG, except that in SFG the 
two input waves are at different frequencies. One application of the SFG process is to obtain tunable radiation in the UV spectral region by choosing a fixed frequency of an IR laser as the input wave and the output of a tunable laser system in the visible region of the spectrum as the other frequency. Similarly, the process of DFG can be used to produce infrared radiation by mixing the output of a frequency-tunable visible laser with that of a fixed-frequency UV laser.

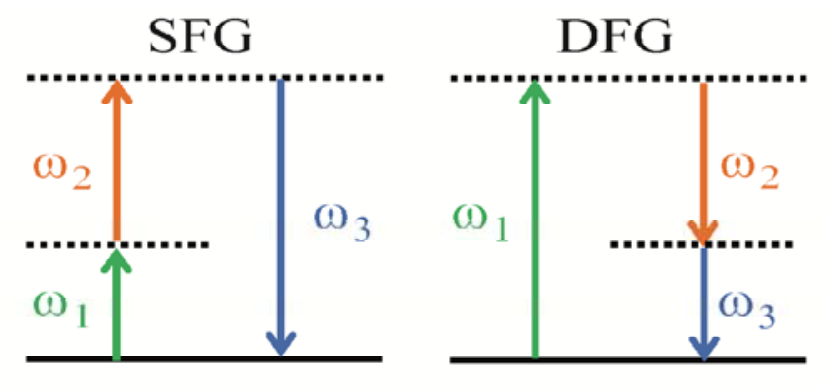

Figure 2.4. Energy-level description of the process of sum (left) and difference-frequency generation (right).

At first sight, SFG and DFG seem to be very similar processes, but an important difference between them can be seen on comparing their energy-level description in Figure 2.4. The DFG process requires that for each photon created at the difference frequency, $\omega_{3}=\omega_{1}-\omega_{2}$, a photon at the higher input frequency, $\omega_{1}$, must be destroyed, and a photon at the lower input frequency, $\omega_{2}$, must be created. Thus, the lower frequency input field, $\omega_{2}$ is amplified by this process. For this reason, the process of DFG is also known as optical parametric amplification, as we shall see later.

If we return to the photon energy-level description of the DFG process, the atom first absorbs a photon of frequency $\omega_{1}$ and then jumps to the highest virtual level. This level decays through a twophoton emission process that is stimulated by the presence of the $\omega_{2}$ field, which is already present. This two-photon emission process can occur even if the $\omega_{2}$ field is not applied; the fields generated in such a case are much weaker, since they are created by a spontaneous two-photon emission from a virtual level. This process is termed, parametric generation or parametric fluorescence, first observed experimentally by Harris et. al. in 1967 [32]. This process cannot be explained under the perturbative, classical description and must be described quantum-mechanically. We shall study this process in more detail in Chapter 7.

\subsubsection{Third-Order Nonlinear Optical Processes}

In this section we shall consider the processes arising from a third-order nonlinear polarization induced in a medium, Eq. (2.4). As can be envisaged from the power of three in the expression, if the incident electric field is made up of several frequency components, the expression for $\tilde{P}^{(3)}(t)$ will be very complicated. Accordingly, we shall consider only one frequency component entering the medium -a monochromatic wave given by Eq. (2.36) - in the same way as we did for the case of the second harmonic. We shall now calculate the third-order polarization, with the result:

$$
\tilde{P}^{(3)}(t)=\varepsilon_{0} \chi^{(3)} E^{3} e^{-i 3 \omega t}+3 \varepsilon_{0} \chi^{(3)}|E|^{2} E e^{-i \omega t}+c c .
$$

The first term in this equation represents the generation of the third harmonic of the incident beam. Note that it depends on the triple of the frequency: $3 \omega$. This process is similar to the process of the 
second harmonic, illustrated in the Figure 2.2. According to a photon description, three photons at frequency $\omega$ will be destroyed and one photon at frequency $3 \omega$ will be created in a single quantummechanical process.

The second term in Eq. (2.42), describes a nonlinear contribution to the polarization at the frequency of the incident field $\omega$. This term thus leads to a nonlinear contribution to the refractive index undergone by a wave at frequency $\omega$. This effect will be important, because the intensitydependent refractive index is responsible for self-focusing, which is the triggering process of supercontinuum generation. We shall explore this further in Chapter 6. At this point we can introduce the notion that the refractive index in the presence of this type of nonlinearity can be represented as:

$$
n=n_{0}+n_{2} I
$$

where $n_{\mathrm{o}}$ is the usual index, i.e., the refractive index at low intensities, and $n_{2}$ is a parameter that characterizes the strength of the nonlinearity, known as the nonlinear refraction index:

$$
n_{2}=\frac{3}{2 n_{0}^{2} \varepsilon_{0} c} \chi^{(3)}
$$

where $I=2 n_{0} \varepsilon_{0} c|E|^{2}$ is the intensity of the incident wave.

To examine a more general form of the third-order polarization, as we did in the case of the second-order polarization (see Eq. (2.41)), one may consider three monochromatic waves inciding on the medium. Considering positive and negative frequencies, one obtains 44 different frequency components, which describe different processes. All the products can be consulted in reference [25]. As an example we shall use the following amplitudes:

$$
\begin{gathered}
P\left(3 \omega_{i}\right)=\varepsilon_{0} \chi^{(3)} E_{i}^{3} \quad \text { with } \quad i=1,2,3 \\
P\left(\omega_{1}+\omega_{2}-\omega_{3}\right)=6 \varepsilon_{0} \chi^{(3)} E_{1} E_{2} E_{3}^{*}
\end{gathered}
$$

The fist one represents the Third-Harmonic Generation (THG) of each of the incoming waves, and the other is associated with a process in which a photon at frequency $\omega_{4}=\omega_{1}+\omega_{2}-\omega_{3}$ is created. It is also instructive to study the form of these equations. In both cases, the frequency argument of the polarization, $P$, is equal to the sum of the frequencies associated with the field amplitudes appearing on the right hand side of the equation, where a negative frequency corresponds to a complex conjugate of the field amplitude. Also, the numerical factor is equal to the number of permutations of the field frequencies that contribute to that term.

\subsubsection{Parametric versus Non-parametric Processes}

We shall use the word parametric along the thesis, and hence we have considered it to be of importance to distinguish the difference between parametric and non-parametric processes. The term parametric denotes a process in which the initial and final quantum-mechanical states of the material system are identical. Consequently, in a parametric process the population of atoms can be removed from the ground state only for the brief intervals of time when it resides at the virtual 
level. Conversely, non-parametric processes involve the transfer of the population of atoms from one real level to another.

An important difference between parametric and non-parametric processes, in terms of their theoretical treatment, is that parametric processes can be described by a real susceptibility; conversely non-parametric processes are described by a complex susceptibility. Another difference is that the photon energy is always conserved in a parametric process; photon energy does not need to be conserved in a non-parametric process because the energy can be transferred to or from the material system.

$\underline{\text { Parametric }}$

- Second-harmonic generation

- Sum- and Difference-frequency generation

- Optical parametric amplification

- Pockels Effect

- Optical Kerr Effect (the intensitydependent refractive index)

- Third-harmonic generation

- Four-wave mixing

\section{Non-parametric}

- Saturable absorption

- Two-photon absorption

- Stimulated Raman scattering

- Ionization

- Multiphoton ionization

- ...

A description of all these processes can be obtained in reference [25].

\subsection{The Coupled Wave Equations for SFG}

In the next section we shall see how the nonlinear optical wave equation that we derived in Sect. 2.3 can be used to describe specific nonlinear optical interactions. In particular we shall start with SFG, a process introduced in Sect. 2.4.2, in which photons at frequencies $\omega_{1}$ and $\omega_{2}$ are destroyed and a photon at frequency $\omega_{3}$ is created, see Figure 2.4 (left). We shall consider a lossless, nonlinear optical medium involving collimated, monochromatic, continuous-wave input beams. We adopt the configuration presented in Figure 2.3, where the applied waves enter the nonlinear medium at normal incidence and we ignore double-refraction effects[25].

Wave equation (2.35) must hold for each frequency component of the field, and in particular for the sum-frequency component oscillating at $\omega_{3}$. In the absence of a nonlinear source term $\left(\tilde{P}^{(N L)}\right.$ $=0$ ), the solution to this equation is a plane wave at frequency $\omega_{3}$ propagating in the forward direction:

$$
E_{3}(z, t)=\mathcal{A}_{3} e^{i\left(k_{3} z-\omega t\right)}+c c
$$

where

$$
k_{3}=\frac{n_{3} \omega_{3}}{c}, \quad n_{3}^{2}=\varepsilon^{(1)}\left(\omega_{3}\right)
$$

In this simple case, the amplitude of the wave $\mathcal{A}_{3}$ is a constant.

However, if the nonlinearity is not too large, the solutions to the complete equation, i.e., including the nonlinear term, must have the same shape, except that now the amplitude is a function varying slowly with $z, A_{3}(z)$. 
We shall study the situation in which the source term is:

$$
\tilde{P}_{3}(z, t)=4 \varepsilon_{0} d_{e f f} E_{1} E_{2} e^{-i\left(\omega_{1}+\omega_{2}\right) t}+c c .=P_{3} e^{-i \omega_{3} t}+c c .
$$

Recall that this was presented in Eq. (2.39) as the source term for SFG. Note that we have introduced the term ${ }^{7} d_{e f f}$, which due to historical convention satisfies $d_{\text {eff }}=\frac{1}{2} \chi^{(2)}$. The applied electric fields will be in the form $(i=1,2)$ :

$$
\tilde{E}_{i}(z, t)=E_{i} e^{-i \omega_{i} t}+c c . \text { where } E_{i}=A_{i}(z) e^{i k_{i} z} .
$$

Therefore, the amplitude of the nonlinear polarization can be written as

$$
P_{3}=4 \varepsilon_{0} d_{e f f} A_{1} A_{2} e^{i\left(k_{1}+k_{3}\right) z} .
$$

Now introducing Eqs. (2.47), (2.49), (2.51) in (2.35), we obtain the following wave equation:

$$
\begin{aligned}
& {\left[\frac{d^{2} A_{3}}{d z^{2}}+2 i k_{3} \frac{d A_{3}}{d z}-k_{3}^{2} A_{3}+\frac{\varepsilon^{(1)}\left(\omega_{3}\right) \omega_{3}^{2} A_{3}}{c^{2}}\right] e^{i\left(k_{3} z-\omega_{3} t\right)}+c c} \\
& =-\frac{4 d_{e f f} \omega_{3}^{2}}{c^{2}} A_{1} A_{2} e^{i\left[\left(k_{1}+k_{2}\right) z-\omega_{3} t\right]}+c c
\end{aligned}
$$

where the $\nabla^{2}$ has been replaced by $d^{2} / d z^{2}$ because the fields depend only on the longitudinal coordinate $z$. Since $k_{3}^{2}=\varepsilon^{(1)}\left(\omega_{3}\right) \omega_{3}^{2} / c^{2}$, the third and fourth terms on the left hand side of this expression are cancelled; also, the conjugate terms can be forgotten at this point. The temporal exponential $\exp \left(-i \omega_{3} t\right)$ can also be cancelled and we obtain the following simplified equation:

$$
\frac{d^{2} A_{3}}{d z^{2}}+2 i k_{3} \frac{d A_{3}}{d z}=-\frac{4 d_{e f f} \omega_{3}^{2}}{c^{2}} A_{1} A_{2} e^{i\left(k_{1}+k_{2}-k_{3}\right) z}
$$

At this point we can perform the slowly varying amplitude approximation, known as SVEA, and neglect the first term on the right hand side of this equation. This approach is valid if:

$$
\left|\frac{d^{2} A_{3}}{d z^{2}}\right| \ll\left|k_{3} \frac{d A_{3}}{d z}\right|
$$

This condition requires that the fractional change in $A_{3}$ at a distance of the order of an optical wavelength must be much smaller than unity. With this approximation made, Eq. (2.53) becomes:

$$
\frac{d A_{3}}{d z}=\frac{2 i d_{e f f} \omega_{3}^{2}}{k_{3} c^{2}} A_{1} A_{2} e^{i \Delta k z}
$$

Likewise, we can derive equations for the other waves:

\footnotetext{
${ }^{7}$ See Appendix A for a detailed justification of this.
} 


$$
\begin{aligned}
& \frac{d A_{1}}{d z}=\frac{2 i d_{e f f} \omega_{1}^{2}}{k_{1} c^{2}} A_{3} A_{2}^{*} e^{-i \Delta k z} \\
& \frac{d A_{2}}{d z}=\frac{2 i d_{e f f} \omega_{2}^{2}}{k_{2} c^{2}} A_{3} A_{1}^{*} e^{-i \Delta k z},
\end{aligned}
$$

where the quantity

$$
\Delta k=k_{1}+k_{2}-k_{3}
$$

is called the wave-vector (or momentum) mismatch or simply phase mismatch. Equations (2.55), (2.56) and (2.57) form a system of coupled amplitude equations. This system shows how the amplitude of each wave varies as a consequence of its coupling to the other two waves.

Recall that in obtaining this system of coupled wave equations we assumed that the medium is lossless. The practical implication of this is that we can make use of the condition of full permutation symmetry, and the coefficient $d_{\text {eff }}$ has the same value in each equation. See Appendix A for further details.

\subsubsection{Considerations about Phase Matching}

The next step is to assume that the amplitudes of the input fields can be taken as constant on the right-hand side of Eq. (2.55). This means that the conversion of the input fields into the sumfrequency is small. This approximation is known as the undepleted input beam approximation, or the undepleted pump. Note that for the especial case:

$$
\Delta k=0
$$

the amplitude of the wave generated, $A_{3}$, increases linearly with $z$ and consequently its intensity increases quadratically with $z$. This condition (Eq. (2.59)) is known as the condition of perfect phase matching. When this condition is fulfilled, the wave generated maintains a fixed phase relation with respect to the nonlinear polarization and is able to extract energy more efficiently from the incident waves.

From a microscopic point of view, when the condition of perfect phase matching is fulfilled the individual atomic dipoles that constitute the material system are properly phased, such that the field emitted by each dipole adds constructively in the forward direction.

As expected, for the reasons given above, when this perfect phase-matching condition is not satisfied the intensity of the emitted radiation is smaller than for the case when $\Delta k=0$. Maintaining the approximation of constant amplitudes, $A_{1}$ and $A_{2}$, the amplitude at an exit plane $L$ can be calculated by integrating Eq. (2.55) from the beginning, $z=0$, to the end of the medium, $z=L$, for the more general case of $\Delta k \neq 0$.

$$
A_{3}(L)=\frac{2 i d_{e f f} \omega_{3}^{2} A_{1} A_{2}}{k_{3} c^{2}} \int_{z=0}^{z=L} e^{i \Delta k z} d z=\frac{2 i d_{e f f} \omega_{3}^{2} A_{1} A_{2}}{k_{3} c^{2}}\left(\frac{e^{i \Delta k L}-1}{i \Delta k}\right)
$$

After some calculations, the intensity of the field at the output can be expressed as:

$$
I_{3}=\frac{8 d_{e f f}^{2} \omega_{3}^{2} I_{1} I_{2}}{n_{1} n_{2} n_{3} \varepsilon_{0} c^{2}} L^{2} \operatorname{sinc}^{2}\left(\frac{\Delta k L}{2}\right)
$$


where the intensity of each of the waves is given by the magnitude of the time-averaged Poynting vector, which for our definition of the field amplitude is given by:

$$
I_{i}=2 n_{i} \varepsilon_{0} c A_{i} A_{i}^{*}, \quad i=1,2,3
$$

We can rewrite equation (2.61) to make the influence of the phase mismatch on the intensity of the generated wave more evident:

$$
I_{3}=I_{3}^{(\max )}\left[\frac{\sin (\Delta k L / 2)}{(\Delta k L / 2)}\right]^{2}
$$

where $I_{3}^{(\max )}$ is the maximum value of intensity, $I_{3}$, which corresponds to a wave-vector situation in which the condition of perfect phase matching is satisfied. The intensity decays in the rest of the situations, as can be seen from this latter equation. Note that the effect of wave-vector mismatching is included in the factor $\operatorname{sinc}^{2}(\Delta k L / 2)$, which is known as the phase-mismatch factor and is plotted in Figure 2.5.

It should be noted that the efficiency of the SFG process decreases as $|\Delta k| L$ increases, with the occurrence of some oscillations. The reason for this behaviour is that if $L$ is greater than approximately $1 / \Delta k$, the output may become dephased with respect to its driving polarization, and power can flow from the generated wave, $\omega_{3}$, to the other two waves. This phenomenon is known as backconversion. Therefore, when the condition of perfect phase matching is fulfilled the intensity of the $I_{3}$ wave scales with $L^{2}$, but when the phase matching is not zero the intensity oscillates with a period of $(2 \pi / \Delta k)$. See Eq. (2.61).

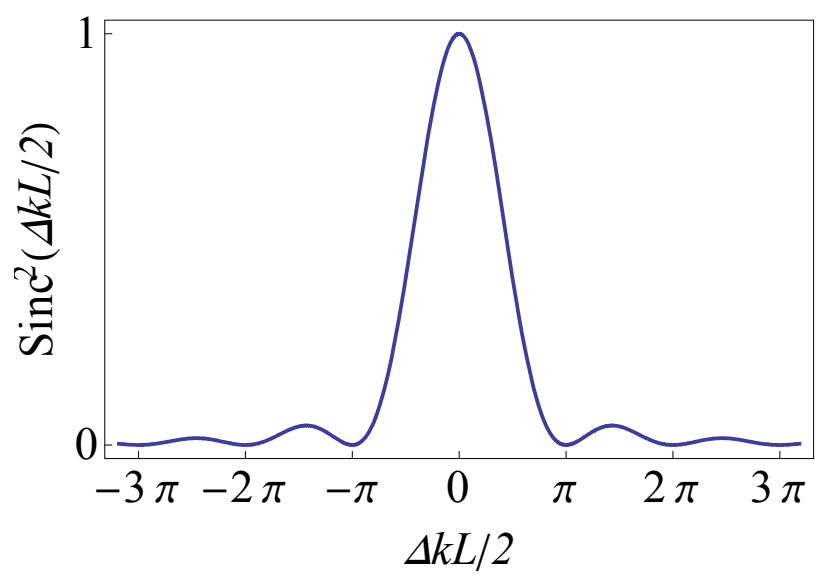

Figure 2.5. Effect of wave-vector mismatching on the efficiency of SFG.

\subsubsection{Solutions Beyond the Undepleted Pump Approximation}

We can solve the coupled wave equations, Eqs. (2.55) to (2.57), numerically without the need to make the approximation of the undepleted pump wave. An analytical solution of the equations can be consulted in reference [33]. In Figure 2.6, we plot the numerical solution of those equations; it is possible to see the variation in the intensity of the interacting waves along the nonlinear medium (a BBO crystal) for different initial conditions. 
In the case of perfect phase matching, Figure 2.6 a), and with an equal number of photons in the incoming waves, it may be seen how at the end of the crystal the whole population in the input waves has been transferred to the generated one, and no backconversion can occur.

a)

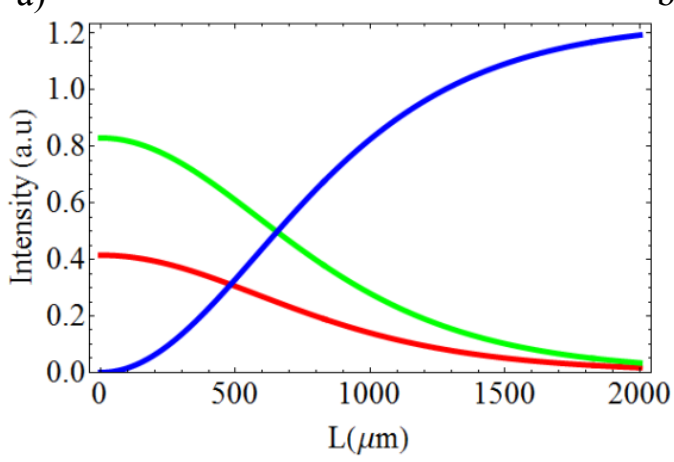

b)

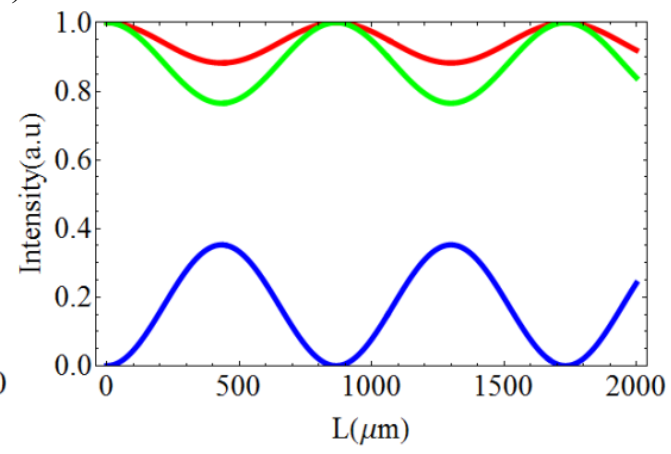

Figure 2.6. Variations in the intensities of the waves undergoing the process of SFG in a $B B O$ nonlinear crystal. In both figures, the blue line represents the wave generated at frequency $\omega_{3}$, which is zero in $L=0$. The red and the green lines are the incoming waves at frequencies $\omega_{1}$ and $\omega_{2}$ respectively. In panel a) the initial condition is an equal number of photons of the $\omega_{1}$ and $\omega_{2}$ waves, and the condition of perfect phase matching is fulfilled. In panel b) the input waves initially have the same intensity and there is a phase mismatch.

However, in the case with the phase mismatch an oscillatory behaviour is clear. The process is not as efficient as in the perfect phase-matching case. Part of the population is transferred to the wave at $\omega_{3}$ in the presence of the other two, which are not completely depleted. As can be seen, once a maximum in the population of the $\omega_{3}$ wave has been obtained photons can be transferred from the wave generated back to the other two waves and this results in the observed oscillating behaviour.

\subsection{The Manley-Rowe Relations}

Taking into account the definition of the intensity discussed in the previous section, Eq. (2.62), we can define the spatial variation of intensity with the propagation direction of each of the interacting waves as [25]:

$$
\frac{d I_{i}}{d z}=2 n_{i} \varepsilon_{0} c\left(A_{i}^{*} \frac{d A_{i}}{d z}+A_{i} \frac{d A_{i}^{*}}{d z}\right)
$$

And making used of the coupled amplitude equations: (2.55), (2.56) and (2.57), we thus obtain:

$$
\begin{aligned}
& \frac{d I_{1}}{d z}=-8 \varepsilon_{0} d_{e f f} \omega_{1} \operatorname{Im}\left(A_{3} A_{1}^{*} A_{2}^{*} e^{-i \Delta k z}\right) \\
& \frac{d I_{2}}{d z}=-8 \varepsilon_{0} d_{e f f} \omega_{2} \operatorname{Im}\left(A_{3} A_{1}^{*} A_{2}^{*} e^{-i \Delta k z}\right) \\
& \frac{d I_{3}}{d z}=8 \varepsilon_{0} d_{e f f} \omega_{1} \operatorname{Im}\left(A_{3} A_{1}^{*} A_{2}^{*} e^{-i \Delta k z}\right)
\end{aligned}
$$

At first glance, we see how the variation in $I_{1}$ and $I_{2}$ have the same sign and that it is opposite to the variation in $I_{3}$. This set of equations demonstrates that the total power flow is conserved, as 
expected for propagation in a lossless medium. However, to demonstrate this, we can define the total intensity as:

$$
I=I_{1}+I_{2}+I_{3}
$$

And from the above equations, we see how the spatial variation of the total intensity is given by:

$$
\frac{d I}{d z}=-8 \varepsilon_{0} d_{e f f}\left(\omega_{1}+\omega_{2}-\omega_{3}\right) \operatorname{Im}\left(A_{3} A_{1}^{*} A_{2}^{*} e^{-i \Delta k z}\right)=0
$$

Note that the latter equation is equivalent to zero because $\omega_{3}=\omega_{1}+\omega_{2}$. The set of equations (2.65) also implies:

$$
-\frac{d}{d z}\left(\frac{I_{1}}{\omega_{1}}\right)=-\frac{d}{d z}\left(\frac{I_{2}}{\omega_{2}}\right)=\frac{d}{d z}\left(\frac{I_{3}}{\omega_{3}}\right)
$$

These equalities are known as the Manley-Rowe relations. These relations tell us that the rate at which photons, at frequency $\omega_{1}$, are destroyed is equal to the rate at which photons at frequency $\omega_{2}$ are destroyed, and it is equal to the rate at which photons at frequency $\omega_{3}$ are created. This result can be understood intuitively by means of the diagram to the left of Figure 2.4 (SFG). We also anticipated this in the discussion made in Sect. 2.5.2.

\subsection{Phase Matching and Nonlinear Crystals}

The perfect phase-matching condition, $\Delta k=0$, is often difficult to achieve because the refractive index of materials that are lossless in the $\omega_{1}$ to $\omega_{3}$ range (assuming $\omega_{1} \leq \omega_{2}<\omega_{3}$ ) shows an effect known as normal dispersion; i.e. the refractive index increases with frequency. As a result the condition for perfect phase matching with collinear beams:

$$
\frac{n_{1} \omega_{1}}{c}+\frac{n_{2} \omega_{2}}{c}=\frac{n_{3} \omega_{3}}{c}
$$

where $\omega_{1}+\omega_{2}=\omega_{3}$, cannot be achieved. For the case of SHG where $\omega_{1}=\omega_{2}, \omega_{3}=2 \omega_{1}$, this condition requires that:

$$
n\left(\omega_{1}\right)=n\left(2 \omega_{1}\right)
$$

which is clearly not possible when $n$ increases monotonically with $\omega$. For the case of the SFG process, the argument is slightly more complicated but the conclusion is the same.

The most common procedure to achieve phase matching is to make use of the birefringence displayed by many crystals. Birefringence is the dependence of the refractive index on the direction of polarization of the optical radiation. Not all crystals display birefringence; in particular crystals belonging to the cubic crystal system are optically isotropic and thus not phase-matchable. Glasses and amorphous materials are also non-birefringent.

However, uniaxial crystals are suitable for birefringent phase-matching. One of them is BBO, which in particular is a negative uniaxial crystal. 
Uniaxial crystals are characterized by a special direction called the optical axis, which we shall refer to below as the c-axis. The plane containing this axis and the wave-vector $\mathbf{k}$, the propagation direction of light, is termed the principal plane. The light beam whose polarization (i.e. oscillations of the electric field vector $\mathbf{E}$ ) is normal to the principal plane is called the ordinary beam, or obeam. Such light is subject to the ordinary refractive index $n_{0}$. In contrast, the beam polarized in the principal plane is called the extraordinary beam, or e-beam, and is subject to a refractive index $n_{\mathrm{e}}(\theta)$ that depends on the angle $\theta$ between the optical axis and the wave-vector, $\mathbf{k}$. The value of this index can be calculated through the relation:

$$
\frac{1}{n_{e}(\theta)^{2}}=\frac{\sin ^{2} \theta}{n_{z}^{2}}+\frac{\cos ^{2} \theta}{n_{o}^{2}}
$$

Here, $n_{z}$ is the principal value of the extraordinary refractive index. Note that $n_{e}(\theta)$ is equal to the value of $n_{z}$ when $\theta=90^{\circ}$, and when $\theta=0, n_{e}$ is equal to $n_{o}$. Phase matching is achieved by adjusting the $\theta$ angle to obtain the value of $n_{e}(\theta)$ for which the condition $\Delta k=0$ is satisfied.

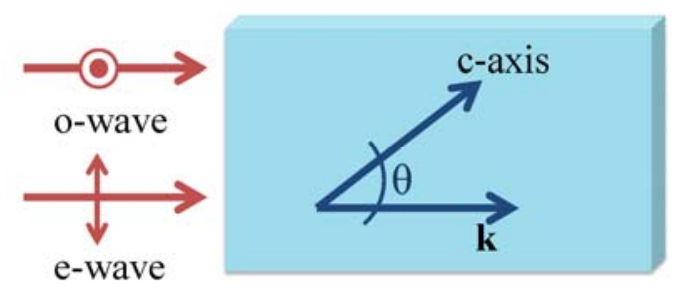

Figure 2.7. Sketch representing the convention for ordinary and extraordinary waves in a uniaxial nonlinear crystal.

In order to achieve phase matching through the use of birefringent crystals, the highest frequency wave, $\omega_{3}=\omega_{1}+\omega_{2}$, is polarized in the direction that gives the lower of the two possible refractive indices. For the case of a negative uniaxial crystal, as is the case of BBO -the one that we shall use in the experiments-, the polarization corresponds to the extraordinary polarization. There are two choices for the polarization of the lowest-frequency waves. Following the definition in [34], Type I phase-matching is the case in which the two lowest-frequency waves have the same polarization, and Type II is the case where the polarizations are orthogonal. The different types of phase matching are synthesized in the following table:

\begin{tabular}{ccc}
\hline & $\begin{array}{c}\text { Positive uniaxial } \\
\left(n_{e}>n_{o}\right)\end{array}$ & $\begin{array}{c}\text { Negative uniaxial } \\
\left(n_{e}<n_{o}\right)\end{array}$ \\
\hline Type I & $n_{3}^{o} \omega_{3}=n_{1}^{e} \omega_{1}+n_{2}^{e} \omega_{2}$ & $n_{3}^{e} \omega_{3}=n_{1}^{o} \omega_{1}+n_{2}^{o} \omega_{2}$ \\
Type II & $n_{3}^{o} \omega_{3}=n_{1}^{o} \omega_{1}+n_{2}^{e} \omega_{2}$ & $n_{3}^{e} \omega_{3}=n_{1}^{e} \omega_{1}+n_{2}^{o} \omega_{2}$ \\
\hline Table 2.1. Phase-matching methods for uniaxial crystals [25].
\end{tabular}

Typically, phase matching is accomplished by one of two methods: angle tuning and temperature tuning. In this work, we shall always use the former. This method involves precise angular orientation of the crystal with respect to the direction of propagation of the incident light. However, the birefringence is temperature-dependent and hence instead of making a rotation of the 
crystal it is possible to phase-match the mixing process by holding $\theta$ fixed at $90^{\circ}$ and varying the temperature of the crystal.

Below, we shall see the angle tuning method in detail for the case of SHG in a uniaxial crystal. From Table 1, it is easy to see that the Type I phase-matching condition becomes:

$$
n_{e}(2 \omega, \theta)=n_{o}(\omega)
$$

with $\omega_{1}=\omega_{2}=\omega$ and $\omega_{3}=2 \omega$. Making use of equation (2.71) and the above relation of indices, one can calculate the phase-matching angle for SHG, or in other words, how the crystal should be oriented in order to achieve the phase-matching condition.

$$
\sin ^{2} \theta_{P M}=\frac{\frac{1}{n_{o}(\omega)^{2}}-\frac{1}{n_{o}(2 \omega)^{2}}}{\frac{1}{n_{z}(2 \omega)^{2}}-\frac{1}{n_{o}(2 \omega)^{2}}}
$$

The following figure illustrates the process of angle tuning in BBO for the particular case of the generation of the second harmonic of a monochromatic wave in the IR region of the spectrum, 800 $\mathrm{nm}$. The radiation obtained would be in the near UV region: $400 \mathrm{~nm}$.

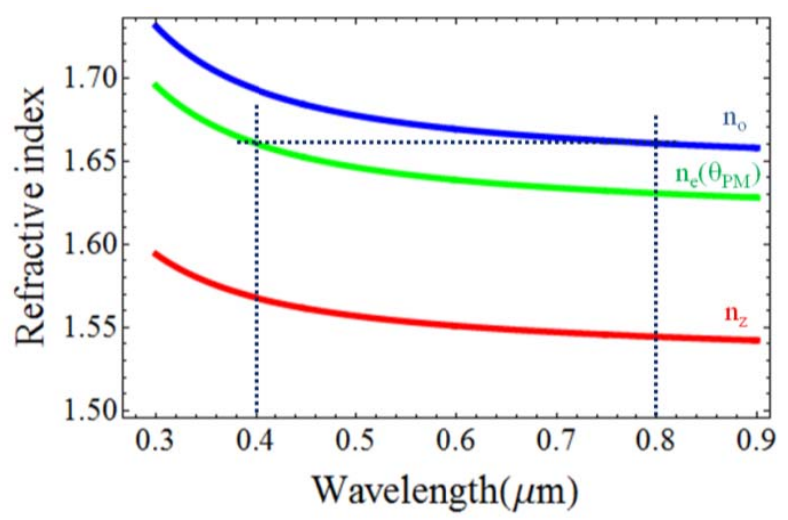

Figure 2.8. Representation of the dispersion functions for the refractive indices: ordinary, $n_{o}$, extraordinary, $n_{e}\left(\theta_{P M}\right)$ and principal, $n_{z}$. The extraordinary index function corresponds to the $\theta$ value to phase-match Type I $S H G$ at $800 \mathrm{~nm}$.

\subsection{The DFG Process}

This is also termed parametric down-conversion. We shall now study the process of DFG; the situation where light waves at frequency $\omega_{3}$ and at frequency $\omega_{1}$ enter a lossless, nonlinear medium to produce an output wave at the difference frequency $\omega_{2}=\omega_{3}-\omega_{1}[25]$.

We shall consider that the wave at frequency $\omega_{3}$ is a strong wave, as we shall see later. We shall solve the coupled amplitude equations under the approximation of the undepleted pump wave $\left(d A_{3} / d z=0\right)$. We also assume that no field is incident on the medium at the frequency $\omega_{2}$. This is also a common situation in experimental work. 


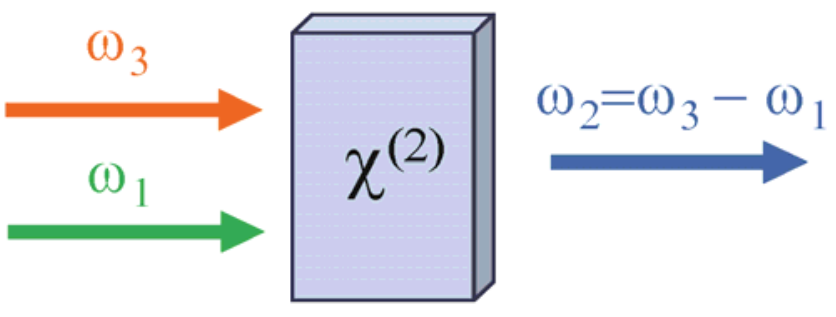

Figure 2.9. Sketch representing the DFG process.

The coupled amplitude equations describing this interaction can be obtained in a similar way to what was done for the coupled wave equation for SFG. The equations for the varying amplitudes $A_{1}$ and $A_{2}$ take the form of:

$$
\begin{aligned}
& \frac{d A_{1}}{d z}=\frac{2 i \omega_{1}^{2} d_{e f f}}{k_{1} c^{2}} A_{3} A_{2}^{*} e^{i \Delta k z} \\
& \frac{d A_{2}}{d z}=\frac{2 i \omega_{2}^{2} d_{e f f}}{k_{2} c^{2}} A_{3} A_{1}^{*} e^{i \Delta k z}
\end{aligned}
$$

where

$$
\Delta k=k_{3}-k_{1}-k_{2}
$$

Again, we solve this equation for the case of perfect phase matching: $\Delta k=0$ [35]. The procedure is as follows: first, we differentiate the second equation of the system with respect to $z$ and introduce the complex conjugate in the first equation to eliminate $d A_{1}^{*} / d z$ from the right hand side. We thus obtain the simplified differential equation:

$$
\frac{d^{2} A_{2}}{d z^{2}}=\frac{4 \omega_{1}^{2} \omega_{2}^{2} d_{e f f}^{2}}{k_{1} k_{2} c^{4}}\left|A_{3}\right|^{2} A_{2} \equiv \kappa^{2} A_{2}
$$

where $\kappa$ is a real coupling constant. The general solution to this equation is:

$$
A_{2}(z)=C \sinh \kappa z+D \sinh \kappa z
$$

Applying the boundary conditions $A_{2}(0)=0$, and where $A_{1}(0)$ has an arbitrary value, the solutions to the set of equations (2.74) that meet the boundary conditions are:

$$
\begin{aligned}
& A_{1}(z)=A_{1}(0) \cosh \kappa z \\
& A_{2}(z)=i\left(\frac{n_{1} \omega_{2}}{n_{2} \omega_{1}}\right)^{1 / 2} \frac{A_{3}}{\left|A_{3}\right|} A_{1}^{*}(0) \sinh \kappa z
\end{aligned}
$$

From this solution we should note that both the $\omega_{1}$ and the $\omega_{2}$ fields experience a monotonic growth and that asymptotically (i.e. for $k z \gg 1$ ) each field experiences an exponential growth. From the form of the solution we can see how $A_{l}$ maintains its initial phase and is simply amplified by the interaction, whereas the wave generated at frequency $\omega_{2}$ has a phase that depends on both that of 
the pump and on that of the $\omega_{1}$ wave. This behaviour of the monotonic growth of both waves is qualitatively dissimilar to that of SFG, in which oscillatory behaviour occurs.
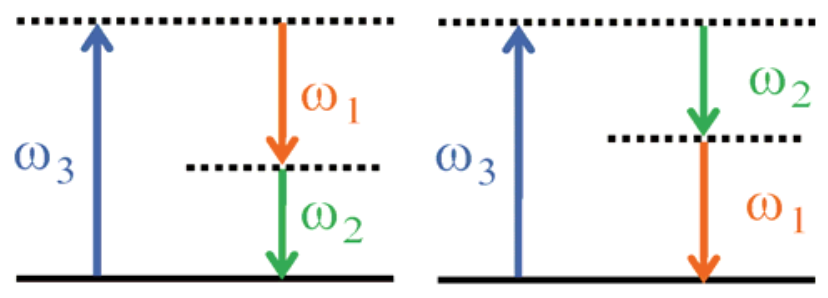

Figure 2.10. Energy-level description of the DFG process.

In this case, the reason for the different behaviour can be understood in terms of the energy-level diagram shown in the above figure. In the left panel we see how the presence of a field at frequency $\omega_{1}$ stimulates the downward transition that leads to the generation of the $\omega_{2}$ field. Likewise, if we look at the right panel we see how the presence of the $\omega_{2}$ field stimulates the generation of the $\omega_{1}$ field. Hence, and this is the main idea, the generation of the $\omega_{1}$ field reinforces the generation of the $\omega_{2}$ field and vice versa, thus leading to the exponential growth of each wavelength.

Since the $\omega_{1}$ field is amplified by the process of DFG, which is a parametric process, this process is also known as parametric amplification. Hereafter, the $\omega_{1}$ field will be called the signal wave, which will be amplified in the process of DFG, and the $\omega_{2}$ field generated will be the idler wave.

In the next figure we represent the numerical solution of the coupled wave equations, Eq. (2.74), but without the undepleted pump approximation, as we did for the process of SFG. The analytic resolution of the coupled wave equations can be consulted in reference [36].
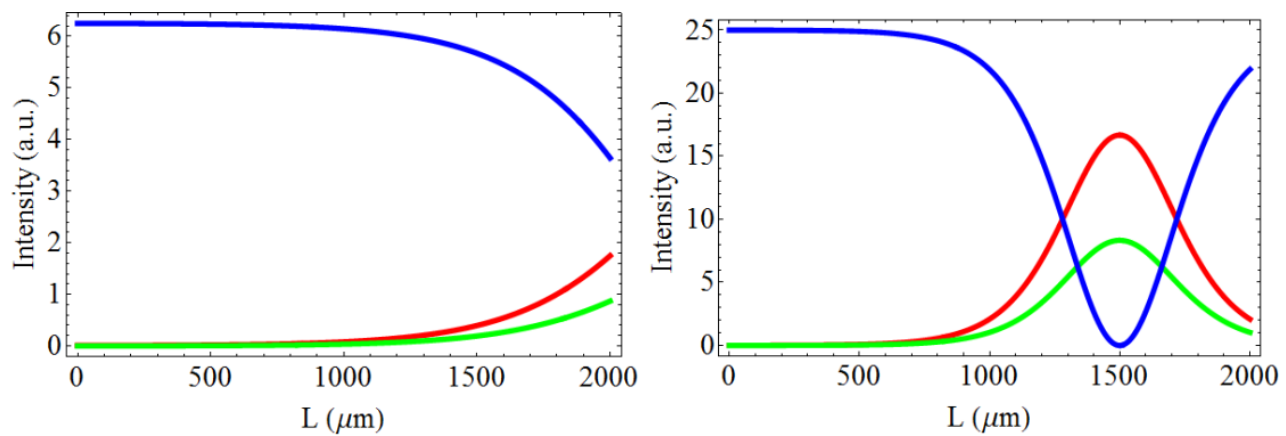

Figure 2.11. Solutions to the coupled wave equations of DFG without the undepleted pump approximation. In both figures, the blue line corresponds to the intensity of the pump wave; the red line represents the signal wave, and the green line corresponds to the idler wave. The panel on the left corresponds to the situation where the pump wave is not very intense, while the right panel corresponds to a very intense pump wave. In both cases, the condition of perfect phase matching is fulfilled.

In the left panel in Figure 2.11 we see how the two waves are amplified due to the presence of the pump wave. However, if we compare it with the more intense case (right panel), we see how the maximum amplification is rapidly achievable and the energy is converted back to the pump wave. 
In both cases, the condition of perfect phase matching is fulfilled; if this were not the case, we would see an oscillatory behaviour.

Let us recall that OPA differs from DFG only in the initial conditions. In this case, the signal beam is much weaker than the pump beam, and the signal becomes significantly amplified during the interaction, while the idler is generated at the same time.

If we solve the coupled wave equations that describe this process, Eqs. (2.74), as before, neglecting pump depletion, with the same initial conditions but in a more general case of phase mismatch different from zero, we can obtain the signal and idler intensities after a length, $L$, of the nonlinear crystal as [35]:

$$
\begin{aligned}
& I_{s}(L)=I_{s}(0)\left[1+\frac{\Gamma^{2}}{g^{2}} \sinh ^{2}(g L)\right] \\
& I_{i}(L)=I_{s}(0) \frac{\omega_{i}}{\omega_{s}} \frac{\Gamma^{2}}{g^{2}} \sinh ^{2}(g L)
\end{aligned}
$$

where

$$
g=\sqrt{\Gamma^{2}-\left(\frac{\Delta k}{2}\right)^{2}} \quad \text { and } \quad \Gamma^{2}=\frac{8 \pi^{2} d_{e f f}^{2} I_{p}}{n_{i} n_{s} n_{p} \lambda_{i} \lambda_{p} \varepsilon_{0} c}
$$

in the case of perfect phase matching $(\Delta k=0, \mathrm{~g}=\Gamma)$, and within the large-gain approximation ( $\Gamma \mathrm{\gg} \gg 1)$, Eqs (2.79) transform to:

$$
\begin{aligned}
I_{s} & \cong \frac{1}{4} I_{s}(0) \exp (2 \Gamma L) \\
I_{i} & \cong \frac{\omega_{i}}{4 \omega_{s}} I_{s}(0) \exp (2 \Gamma L)
\end{aligned}
$$

These equations show an exponential growth in intensity with crystal length. This reflects something that we have already introduced from the quantum view of the process. Note that the ratio of the signal and idler intensities is such that an equal number of signal and idler photons are generated. We have introduced these equations because (although they are redundant with Eqs. (2.78) ) with them we can define a parametric gain as:

$$
G_{0}=\frac{I_{s}(L)}{I_{s}(0)}=\frac{1}{4} \exp (2 \Gamma L)
$$

The parametric gain grows exponentially with the crystal length $L$ and with the nonlinear coefficient $d_{e f f}$. It is also important to note that the exponential growth of the signal and idler waves along the crystal is qualitatively different from the quadratic growth occurring in other second-order nonlinear phenomena, such as SFG or SHG.

In the next figure we plot the variation in parametric gain as a function of pump intensity for different crystal lengths $L$. Note the extremely high values obtained for not very thick crystals. 


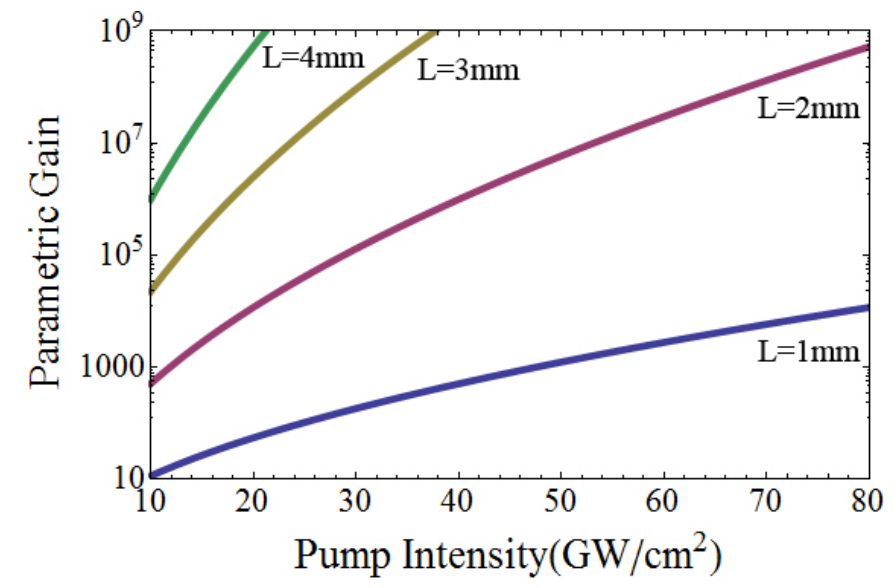

Figure 2.12. Parametric gain as a function of pump intensity for different crystal lengths in $B B O$. 



\section{Chapter 3}

\section{Design, Characterization and Implementation of a Femtosecond SHG/THG Module}

\subsection{Introduction}

The present chapter will be devoted to generating the second and third harmonic of the Ti:Sapphire laser experimentally. Both processes have already been introduced theoretically in the previous chapter, but the treatment was carried out only for monochromatic waves. Here, we shall revise the theory to account for the particular case of working with femtosecond pulses. Thus, the description of the design and implementation of a module for generating the second and third harmonic will serve as an excuse to introduce the theory underlying the generation of these low-order harmonics with femtosecond pulses.

The interest in these phenomena is related to the possibility they offer of expanding the range of emission of conventional laser systems, something that at the same time broadens the range of applications of such lasers. In the case of Ti:Sapphire-based laser systems, the interest in extending the tuning range arose in the early nineties, once laser technology was mature enough to generate IR pulses from regeneratively amplified mode-locked lasers [37], with energies ranging from the $\mu \mathrm{J}$ level to a few tens of $\mathrm{mJ}$, and with pulse durations ranging from 10 to $100 \mathrm{fs}$. At that time, it became of great importance to obtain wavelengths in the visible and near UV regions. However, an 
important issue arose: this was how to maintain the time duration of the original pulses without introducing any significant spectral distortion in the pulses generated. The SHG and SFG processes became important not only for generating frequencies in those regions but also for their application in the pumping of other nonlinear frequency extension devices, such as OPAs, optical parametric generators (OPGs), and optical parametric oscillators (OPOs).

Often, all these processes of frequency conversion lead to pulse broadening and frequency chirping. Consequently, an important issue in SHG (and SFG) with ultrashort pulses is the preservation of pulse duration. Therefore, a detailed analysis is required to achieve highly efficient conversions in both processes without significant degradation in the spectral and temporal characteristics of the converted pulses. Modules like the one we have built exist commercially but having one customized opens more possibilities of adaptability; for example, the possibility of using a different doubling nonlinear crystal when necessary. Also, having these beams available permanently in the laboratory opens the path to new studies in the field of materials microprocessing. Some examples of the applications of this module will be explained in detail in Chapter 8.

The present chapter is organized as follows: in the next section we shall introduce the system of coupled wave equations that describes the propagation of fundamental and second-harmonic pulses in a medium with second- and third-order susceptibilities. We then describe the numerical results obtained after applying such a model to possible experimental situations. In particular, we shall analyze the characteristics of the second-harmonic and fundamental pulses at the output of the nonlinear medium BBO. Also, a similar model will be applied to study the third-harmonic pulses obtained through the SFG process. Following this, we shall present the experimental set-up of the module and we shall continue by describing the design issues we have faced, focusing on the part of choosing the most appropriate crystal. Finally, we present a summary of the most relevant experimental measurements, which include the characterization of two BBO samples of different thickness in terms of conversion efficiency; a temporal and spectral characterization of the secondharmonic pulses generated in those crystals, and finally a characterization of the conversion efficiency in the generation of the third-harmonic pulses.

\subsection{SHG with Femtosecond Pulses. Numerical Model}

In the previous chapter we introduced the wave equation governing the propagation of monochromatic plane waves in a nonlinear medium. Although that theory was sufficient to account for the processes that may arise due to the induced nonlinear polarization, it does not suffice to understand how a femtosecond pulse will propagate inside such a medium. Therefore, in this section we shall introduce a modified equation that includes some parameters that characterize femtosecond pulse propagation, such as the group velocity or the Group-Velocity Dispersion (GVD).

To begin, we take the scalar wave equation that we derived in the previous chapter: Eq (2.35). As stated, we must consider an equation governing the propagation of the drive wave, which hereafter we shall refer to as the fundamental, and another one for its second harmonic. Both waves propagate through a nonlinear crystal with second-order nonlinearity. In the previous chapter we commented on how a medium with second-order susceptibility can also have a non-zero third-order susceptibility. This issue could be included in the model [37], however we consider that at the intensities at which we shall work is unlike that this higher order processes appear. 


$$
\nabla^{2} \tilde{\mathbf{E}}_{i}-\frac{n\left(\omega_{i}\right)^{2}}{c^{2}} \frac{\partial^{2} \tilde{\mathbf{E}}_{i}}{\partial t^{2}}=\frac{1}{\varepsilon_{0} c^{2}} \frac{\partial^{2} \tilde{\mathbf{P}}_{i}^{N L}}{\partial t^{2}}
$$

We shall use the following notation: $\tilde{\mathbf{E}}_{1}$ will be the electric field of the fundamental pulses, and $\tilde{\mathbf{E}}_{2}$ will be the same for the second-harmonic pulses, with central frequencies $\omega_{1}$ and $\omega_{2}$ respectively. Note that these frequencies are related through the relation: $\omega_{2}=2 \omega_{1}$. Solutions to Eqs. (3.1) have the form ${ }^{8}$ :

$$
\begin{aligned}
& \tilde{E}_{1}(z, t)=\frac{1}{2} E_{1} e^{j\left(k_{1} z-\omega_{1} t\right)}+c . c . \\
& \tilde{E}_{2}(z, t)=\frac{1}{2} E_{2} e^{j\left(k_{2} z-2 \omega_{1} t\right)}+c . c .
\end{aligned}
$$

where $E_{i}=E_{i}(r, t), i=1,2$ are slowly varying amplitudes and where $k_{i}=k\left(\omega_{i}\right) i=1,2$.

The next step is to transform the wave equation (3.1) to introduce the dispersion parameters mentioned above. To do so, it is necessary to carry out a Fourier transformation of the wave equation to a frequency space. The derivation can be followed in [27]. Then, we perform a Taylor expansion in the wave-vector, around the pulse central frequency. In our case we need to do this in the two central frequencies of the fundamental and second-harmonic pulses:

$$
k(\omega) \cong k\left(\omega_{i}\right)+\left|\frac{\partial k}{\partial \omega}\right|_{\omega=\omega_{i}}\left(\omega-\omega_{i}\right)+\frac{1}{2}\left|\frac{\partial^{2} k}{\partial \omega^{2}}\right|_{\omega=\omega_{i}}\left(\omega-\omega_{i}\right)^{2}+\ldots
$$

The different terms in the series represent the different parameters that characterize ultrashort pulse propagation. The first term corresponds to the value of the wave-vector at the pulse central frequency, where we can also identify the phase velocity:

$$
k\left(\omega_{i}\right)=\frac{\omega_{i}}{c} n\left(\omega_{i}\right)=\frac{\omega_{i}}{v\left(\omega_{i}\right)}
$$

The next coefficient in the expansion is the inverse of the group velocity:

$$
v_{g}\left(\omega_{i}\right)^{-1}=\left|\frac{\partial k}{\partial \omega}\right|_{\omega=\omega_{i}}
$$

One can also define the group velocity in terms of a refractive index, which is reminiscent of the definition of the phase velocity in the following way $v_{\mathrm{g}}\left(\omega_{\mathrm{i}}\right)=c / n_{\mathrm{gg}}$, where $n_{i g}=n_{i}+\omega_{i}(\partial n / \partial \omega)_{\omega=\omega_{i}}$ is called the group-velocity index.

We stop the expansion in the next term, and neglect higher dispersion orders. In the next term we find the definition of the GVD, which is the second derivative of the wave-vector with frequency:

\footnotetext{
${ }^{8}$ Note that in this chapter we are using $j$ as the imaginary unit for convenience.
} 


$$
g\left(\omega_{i}\right)=G V D=\left|\frac{\partial^{2} k}{\partial \omega^{2}}\right|_{\omega=\omega_{i}}=\left|\frac{\partial}{\partial \omega}\left(\frac{1}{v_{g}}\right)\right|_{\omega=\omega_{i}}=\left|\frac{1}{v_{g}^{2}} \frac{\partial v_{g}}{\partial \omega}\right|_{\omega=\omega_{i}}
$$

The GVD is responsible for the stretching or compression of the pulses when travelling through a dispersive medium. It gives an idea of how different the group velocities of the different frequency components of the field are. This is an important parameter in ultrashort pulses with very broad frequency bandwidths.

After the dispersion terms are included we can make a transformation back to the spatiotemporal domain and the propagation equation results:

$$
\frac{\partial E_{i}}{\partial z}-\frac{j}{2 k_{i}} \frac{\partial^{2} E_{i}}{\partial z^{2}}=-\left[\frac{1}{v_{g}} \frac{\partial}{\partial t}+\left.\frac{j}{2 k_{i} v_{g}^{2}}\left(1-k_{i} \frac{\partial v_{g}}{\partial \omega}\right)\right|_{\omega_{i}} \frac{\partial^{2}}{\partial t^{2}}+\ldots\right] E_{i}+\frac{j \mu_{0} \omega_{i}^{2}}{2 k_{i}} P_{i}^{(N L)}
$$

Thus, invoking the slowly varying envelope approximation, which neglects the second derivatives in space, see Sect. 2.5, we have:

$$
\begin{aligned}
& \frac{\partial E_{1}}{\partial z}=-\left[\frac{1}{v_{1 g}} \frac{\partial}{\partial t}+\left.\frac{j}{2 k_{1} v_{g}^{2}}\left(1-k_{1} \frac{\partial v_{g}^{2}}{\partial \omega}\right)\right|_{\omega_{1}} \frac{\partial^{2}}{\partial t^{2}}+\ldots\right] E_{1}+\frac{j \omega \chi^{(2)}}{2 n_{\omega} c} E_{1}^{*} E_{2} \\
& \frac{\partial E_{2}}{\partial z}=-\left[\frac{1}{v_{2 g}} \frac{\partial}{\partial t}+\left.\frac{j}{2 k_{2} v_{g}^{2}}\left(1-k_{2} \frac{\partial v_{g}^{2}}{\partial \omega}\right)\right|_{\omega_{2}} \frac{\partial^{2}}{\partial t^{2}}+\ldots\right] E_{2}+\frac{j \omega \chi^{(2)}}{2 n_{2 \omega} c} E_{1}^{2}
\end{aligned}
$$

Where we have included the nonlinear polarization as: $\tilde{P}_{N L}=\varepsilon_{0} \chi^{(2)} \tilde{E}^{2}$. In this case, the electric field is the sum of the fundamental and the second harmonic, with $\tilde{E}=\tilde{E}_{1}+\tilde{E}_{2}$. Then, we group the terms oscillating at frequencies $\omega_{1}$ and $2 \omega_{1}$.

The coupled wave equations in (3.8) can be solved numerically to obtain the amplitudes of the fundamental and the second-harmonic pulses at the output of the nonlinear crystal [37].

\subsection{Temporal Characteristics of the Second Harmonic Generated with Femtosecond Pulses.}

Before introducing the solutions obtained from the coupled wave equations, we shall analyze in detail some of the issues involved in the propagation of ultrashort laser pulses. The dispersion parameters, some of them defined in the previous section, help us to determine the consequences of the propagation of ultrashort pulses in dispersive media. Naturally, nonlinear crystals are dispersive media. As mentioned, the transformation of femtosecond pulses due to dispersion becomes more important as the length of the pulses decreases, because of the greater wavelength content. Moreover, some materials are more dispersive than others.

Therefore, when an ultrashort pulse travels through a medium the following spectral phase is obtained: 


$$
\varphi(\omega)=\frac{\omega}{c} n(\omega) L=k(\omega) L
$$

$L$ being the medium thickness. Thus, making use of expressions: (3.3), (3.5) and (3.6) one obtains

$$
\varphi(\omega) \cong \varphi\left(\omega_{i}\right)+\frac{1}{v_{g}\left(\omega_{i}\right)}\left(\omega-\omega_{i}\right) L+\frac{1}{2} g\left(\omega_{i}\right)\left(\omega-\omega_{i}\right)^{2} L+\ldots
$$

Let us first analyse the implications of the GVD, coefficient of the third term in the expression (3.10). We have already mentioned that this term is responsible for a stretching or a compression in time of the pulses when propagating through a dispersive medium. In particular, a high positive value of this term is responsible for the stretching while a negative one would be responsible for compression. When a pulse travels through a medium and acquires this phase factor, regardless of whether it is negative or positive, it is normally considered that we have introduced chirp, which in other words means that there is a linear variation in the instantaneous frequency of the pulse with time. The amount of chirp introduced when the pulse travels a distance $L$ inside the material is given by the Group Delay Dispersion (GDD) parameter, defined as:

$$
G D D=G V D \cdot L
$$

in units of $\mathrm{fs}^{2}$. We cannot avoid this effect; it is always present when pulses travel through a material. However, in the case of nonlinear crystals, this effect becomes important only when the pulses are very short-close to the few-cycle regime- and this is not our case. The GVD introduced by material dispersion is positive, as can be seen in Figure 3.1 where we have plotted the variation in the GVD with wavelength for the nonlinear crystal BBO. The effect is more important as the pulses get shorter because of the highest wavelength content, and if the pulses are centred in the visible or the UV, as can be seen in the figure.

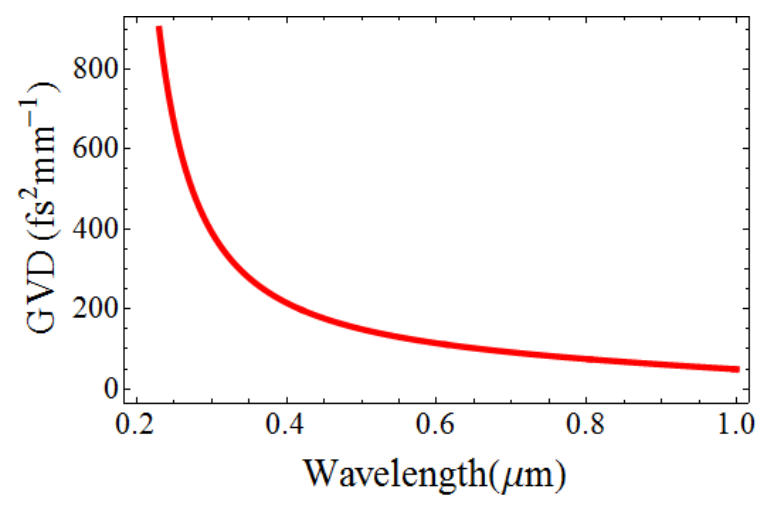

Figure 3.1. GVD as a function of wavelength for an ordinary wave in the nonlinear crystal $B B O$.

As a numerical example, Fourier-transform limited pulses of $120 \mathrm{fs}$, centred either in the IR (800 $\mathrm{nm}$ ) or the blue (400 $\mathrm{nm}$ ), will be increased in time by less than $1 \mathrm{fs}$ when travelling through $2 \mathrm{~mm}$ of BBO [38]. However, pulses with time duration of $30 \mathrm{fs}$ centred in the blue will be increased by $17 \mathrm{fs}$, and $3 \mathrm{fs}$ in the case of being centred in the IR. This shows that the effect of GVD is more important in the visible region of the spectrum, as shown in Figure 3.1. 
We shall continue analyzing the implications of the second term in Eq. (3.10); in particular, the difference in the group velocities of the two interacting pulses. Figure 3.2 shows the variation in the group velocity in terms of wavelength.

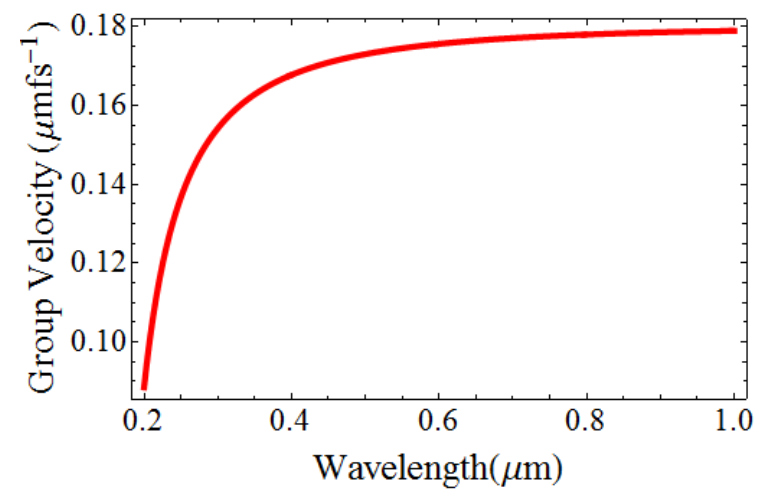

Figure 3.2. Group velocity as a function of wavelength for an ordinary wave in the nonlinear crystal $B B O$.

In regions of normal dispersion, the group velocity of the fundamental is greater than the group velocity of the second harmonic. The difference in the group velocity of the interacting pulses, the blue and the IR, has some important consequences in the generation of second harmonic, as we shall see later in the numerical examples. This difference makes them separate as they propagate through the nonlinear medium, introducing distortions in the temporal shape of the pulses. The parameter that relates the difference in group velocities of two interacting pulses is called the Group Velocity Mismatch, defined as:

$$
G V M=\left|v_{g}(2 \omega)^{-1}-v_{g}(\omega)^{-1}\right|
$$

\subsubsection{Simulations with the SNLO Software}

Theoretical calculations can be performed with a variety of crystal thicknesses, input energies, and peak intensities. However, experimentally we can only study certain situations. Therefore we have chosen the following parameters for the simulations because they are close to laboratory possibilities. The input pulses, fundamental, have a $795 \mathrm{~nm}$ central wavelength, a $120 \mathrm{fs}$ time duration, and a $2.5 \mathrm{~mm}$ pulse diameter (at intensity FWHM criteria). The input peak intensities were chosen as low, medium and high: 7, 40, and $120 \mathrm{GW} / \mathrm{cm}^{2}$ respectively. The crystal thicknesses were $0.5 \mathrm{~mm}$ and $1 \mathrm{~mm}$.

In this case, instead of the implementation of a code to solve the equations (3.8) we have used a resource called SNLO, originally developed at the Sandia laboratories, which can be downloaded from [39]. This software is very easy to use and many types of calculations related to nonlinear optics can be made: SHG, OPA, SFG, etc. The kind of information that can be obtained involves phase-matching bandwidths and refractive indices; all this for a very broad catalogue of nonlinear crystals.

The results are summarized in Figure 3.3. Each figure panel represents the fundamental -red line- and the second-harmonic -blue line- pulses after they propagate through the nonlinear crystals. 


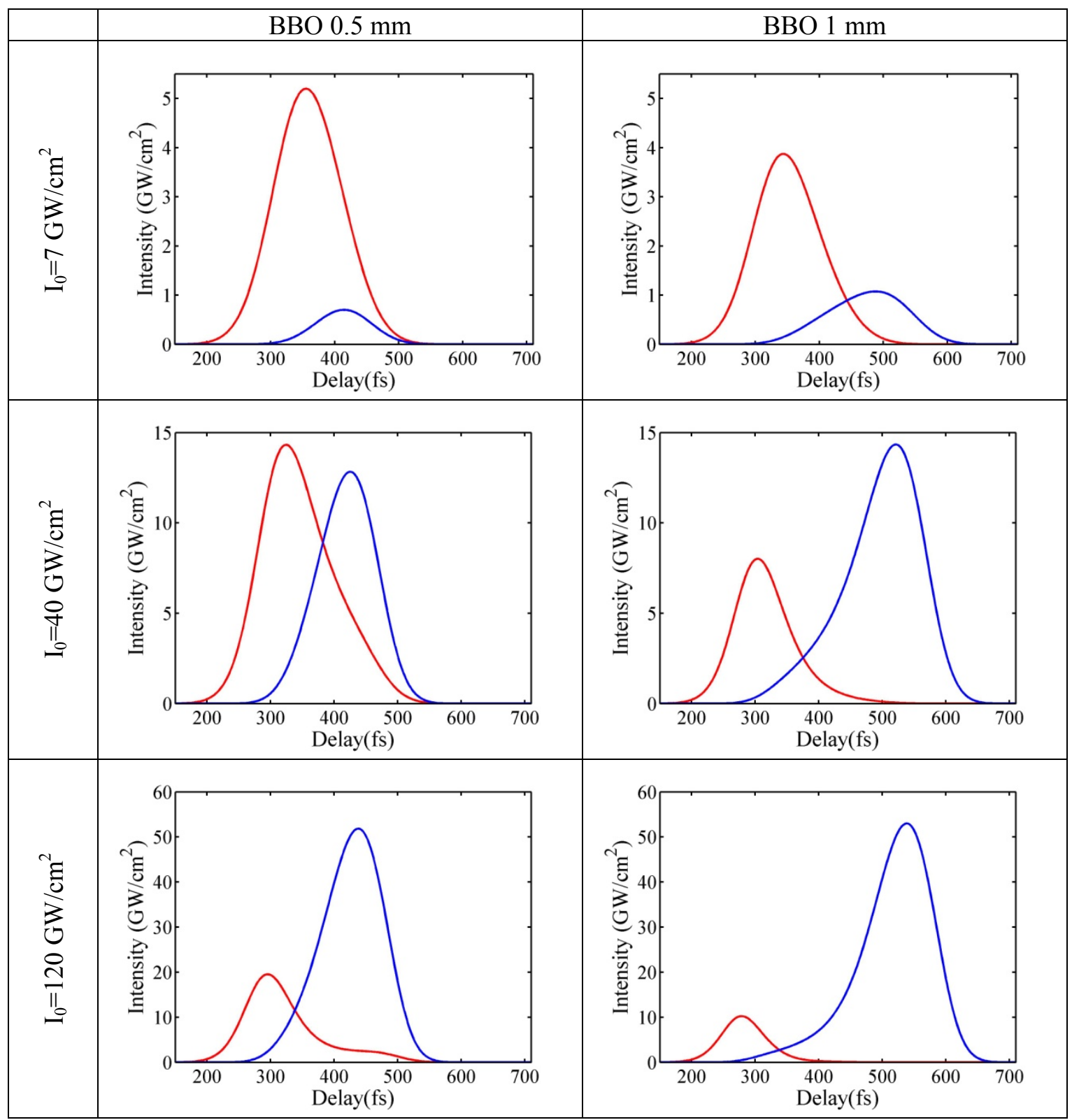

Figure 3.3. Second-harmonic -blue line-and fundamental -red line-pulses at the output of BBO nonlinear crystals. See text for further details.

In the low-intensity case, we can see how the conversion into the blue is low for the two crystals, as expected. However, the longer crystal gives the highest conversion efficiency, $14 \%$ as compared with $4 \%$ for the other one, following the trend described by Eq. $(2.61)^{9}$; i.e., proportional to the square of crystal thickness, $L$. The conversion efficiency was calculated as the energy per pulse in the second harmonic divided by the fundamental's input energy. Observing the duration of the two pulses, we see how the pulse resulting from the thicker crystal is longer in time than the other one. In fact, this pulse becomes lengthened to 155 fs (intensity FWHM), in contrast to the 103 fs time duration for the other crystal. This effect is a direct consequence of the GVM. Once the IR pulses begin to separate from the blue pulses generated, the more intense part of the IR pulse will

\footnotetext{
${ }^{9}$ Although the expression referred to was obtained for the SFG process, it can be easily particularized for the SHG process, as done later in this chapter.
} 
be spatially overlapped with the leading part of the blue ones, and the conversion into second harmonic will be more efficient in this zone, thus elongating the second-harmonic pulse. However, in the case of the thinner crystal the input and generated pulses travel together throughout the crystal.

Finally, if we look into the case with moderate intensity, we see how the temporal characteristics of both pulses are good, being better in the case of the short crystal. The efficiency is only slightly higher for the case of the thicker crystal: $29 \%$ and $15 \%$ for the other one. Again, in the temporal domain the thinner crystal gives the best performance, $110 \mathrm{fs}$ at intensity FWHM, without degradation.

We may conclude that the thin crystal provides the best performance in term of maintaining a good temporal structure and a short duration of the pulses. Nevertheless, a moderate intensity is preferable if we are to avoid temporal effects, above all when using the thicker crystal.

In the case of the highest input energy, the conversion efficiencies are relatively high in both crystals: $32 \%$ for the $1 \mathrm{~mm}$ crystal and $21 \%$ for the other one. However, the tendency of efficiency to increase with crystal thickness is not exactly what would be expected from Eq. (2.61). In terms of peak power, we see how the two crystals give the same value. This behaviour would be expected if we take into account the effect of the GVM. Once the pulses have travelled a certain distance they are separated and the energy cannot be efficiently transferred from the fundamental to the second harmonic. Moreover, a temporal effect can be observed: in the thicker crystal, we see a structure in the leading part of the second-harmonic pulse. This is also a consequence of the GVM, because the intense part of the fundamental will be overlapped with this part of the second harmonic, generating more photons there. This effect is also present in the three energy cases of this crystal, being more important in the last one.

\subsubsection{Temporal Characteristics of the Third Harmonic}

In Sect. 3.2 we introduced the wave equations governing the SHG with femtosecond pulses. Similarly, we could derive a system of coupled wave equations to account for the generation of the third harmonic by means of the SFG process; i.e., as the sum of the fundamental and the second harmonic. Henceforth, when referring to the third harmonic we shall always refer to this process, instead of the one introduced in the previous chapter (Sect. 2.4.3), where the radiation at $3 \omega$ was obtained in a medium with a dominant third-order susceptibility, $\chi^{(3)}$.

Here we present simulations, again made with the SNLO software. In this case, we have maintained fixed the input intensities and simply vary the crystal thickness. The results can be seen in Figure 3.4. For these simulations we used parameters of $795 \mathrm{~nm}$ and $397.5 \mathrm{~nm}$ fundamental and second-harmonic central wavelengths, respectively, a 120 fs time duration of both incoming pulses, and a $2.5 \mathrm{~mm}$ (at intensity FWHM criteria) pulse diameter for the three beams. The input peak intensities were chosen equal for the two input pulses at $20 \mathrm{GW} / \mathrm{cm}^{2}$. The crystal thicknesses taken were 100, 200, 400 and $600 \mu \mathrm{m}$. The results are shown in Figure 3.4.

From the simulations, the first eye-catching effect is that the intensity of the third-harmonic signal is the same in the last three crystals. This is a consequence of the GVM between the interacting pulses, in this case the blue and the IR. The length that these pulses can travel without being completely separated, namely the interaction length, can be estimated as 


$$
l_{\text {int }}=\frac{\tau_{1}+\tau_{2}}{|G V M|}
$$

where $\tau_{1}$ and $\tau_{2}$ are the intensity FWHM time durations of the fundamental and second-harmonic pulses respectively.
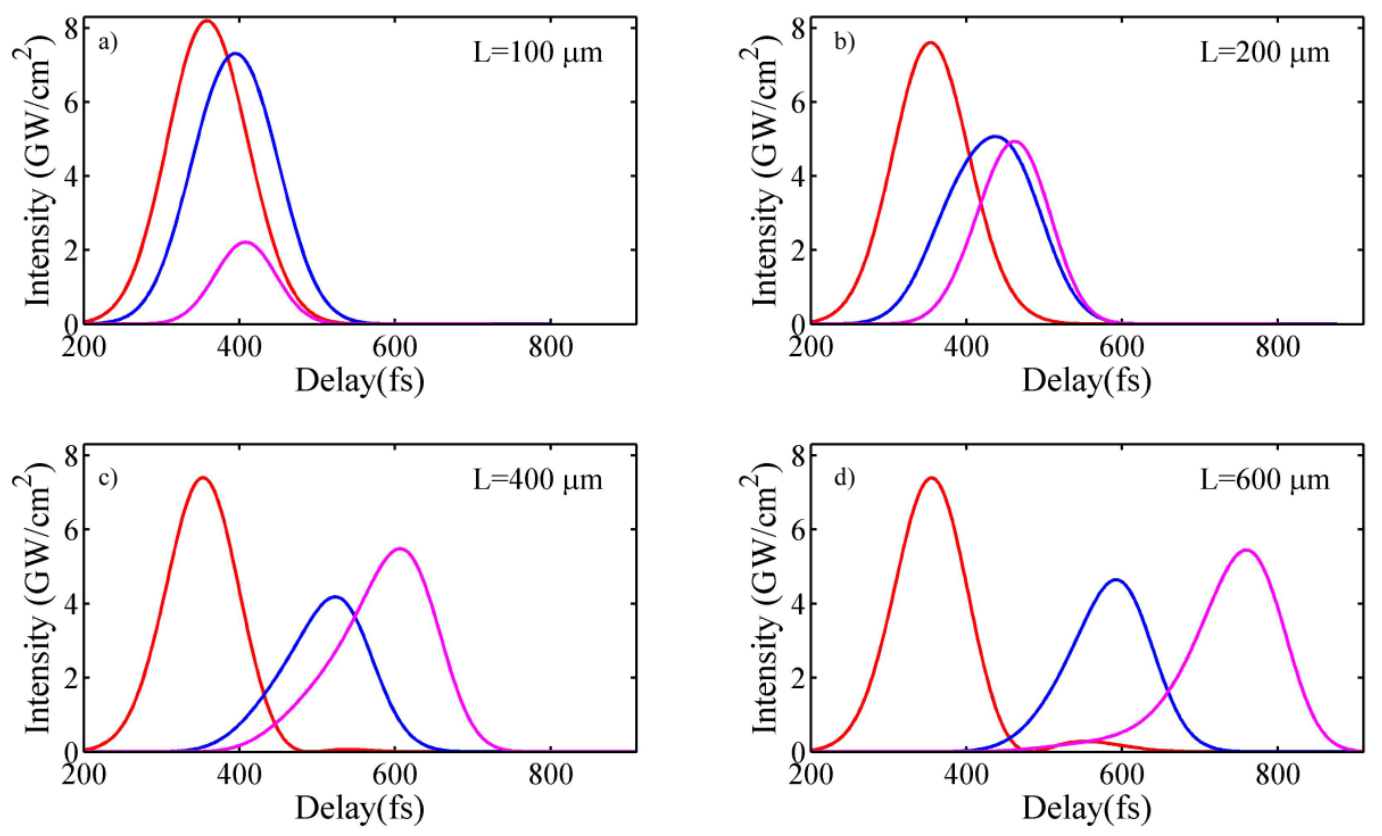

Figure 3.4. Fundamental -red line-, second-harmonic - blue line- and third-harmonicpink line-pulses at the output of the nonlinear crystals with different thicknesses, $L$.

Moreover, a lengthening in the pulse generated can be also observed. Again the GVM, in this case of the UV pulse with the other two, makes it move faster and separate from them, in a similar way to what happened in the case of the second harmonic. We can estimate the lengthening of the pulse in the following way. First, we redefine a GVM to account for the interactions of three waves as:

$$
G V M^{\prime}=\left|v_{g}(3 \omega)^{-1}-v_{g 12}\right|
$$

where $v_{\mathrm{g} 12}$, is the mean value of the group velocities of the second harmonic and fundamental pulses. If we look back to Figure 3.2, we see how the curve drops in the case of the ultraviolet waves, leading them to propagate at a velocity very different from the infrared. Consequently, the lengthening in time due to this effect can be estimated as

$$
\tau_{3}=G V M^{\prime} \cdot l_{\text {int }}
$$

Another interesting result is the energy backconversion from the ultraviolet to the infrared and blue pulses, as can be seen in Figure 3.4 d), This backconversion is more evident in the case of the IR because it occurs in a low-energy part of the pulse, but if we compare the last two panels we can see an increased UV signal with the longer crystal. This effect can be understood as the inverse of the SFG process, where the intense pulse generated now transfers photons to the original pulse. In 
the case of the UV pulses, a photon at frequency $3 \omega$ is split into two photons at frequencies $\omega$ and $2 \omega$ with equal probability.

As already stated, each case needs individual study, and this is why it is very important to make simulations of the propagation of femtosecond pulses in nonlinear media.

\subsection{Experimental Set-Up}

The experiment set-up, depicted in Figure 3.5, consists first of a 1:2 telescope to reduce beam size. As explained in the previous section, there is an optimum intensity -not too low, not too high- for obtaining good conversion efficiency from a nonlinear crystal without introducing undesirable temporal effects in the pulses generated. The reduction in beam size is necessary because with the original size of the beam the input intensities would be too low.

After the telescope, a nonlinear crystal (BBO 1 in Figure 3.5), with cut angles $\theta=29.4^{\circ}, \phi=0^{\circ}$ for Type I SHG, was placed at the output of the BBO 1 crystal. A dichroic beamsplitter (DM1) was used to separate the unconverted fundamental from the generated second harmonic. This kind of mirror has good reflectivity in the blue pulse central wavelength (around $397 \mathrm{~nm}$ ), also having good transmittance for the IR. After the two beams have been split, the red pulses travel to a delay line and after this a half-wave plate is used in order to rotate their polarization. The two beams are collected again by another dichroic mirror (DM2), and they travel collinearly until they reach the second crystal (BBO 2). In this case the crystal is cut to generate the Type I sum-frequency of the IR and the UV pulses $\left(\theta=44.7^{\circ}, \phi=0^{\circ}\right)$. We chose the Type I interaction because the nonlinear constants are higher in this case than in Type II, and therefore conversion efficiency will be higher. See Table 3.1. After the second crystal, the beams can be separated with a prism as indicated in the figure or with a special kind of mirror called a harmonic separator, which works similarly to dichroic mirrors. Using a prism to separate the beams is not valid if we wish to preserve the temporal duration of UV the pulses, which are the ones most affected by material dispersion. Additionally, the prism will introduce spatial $\operatorname{chirp}^{10}[40]$.
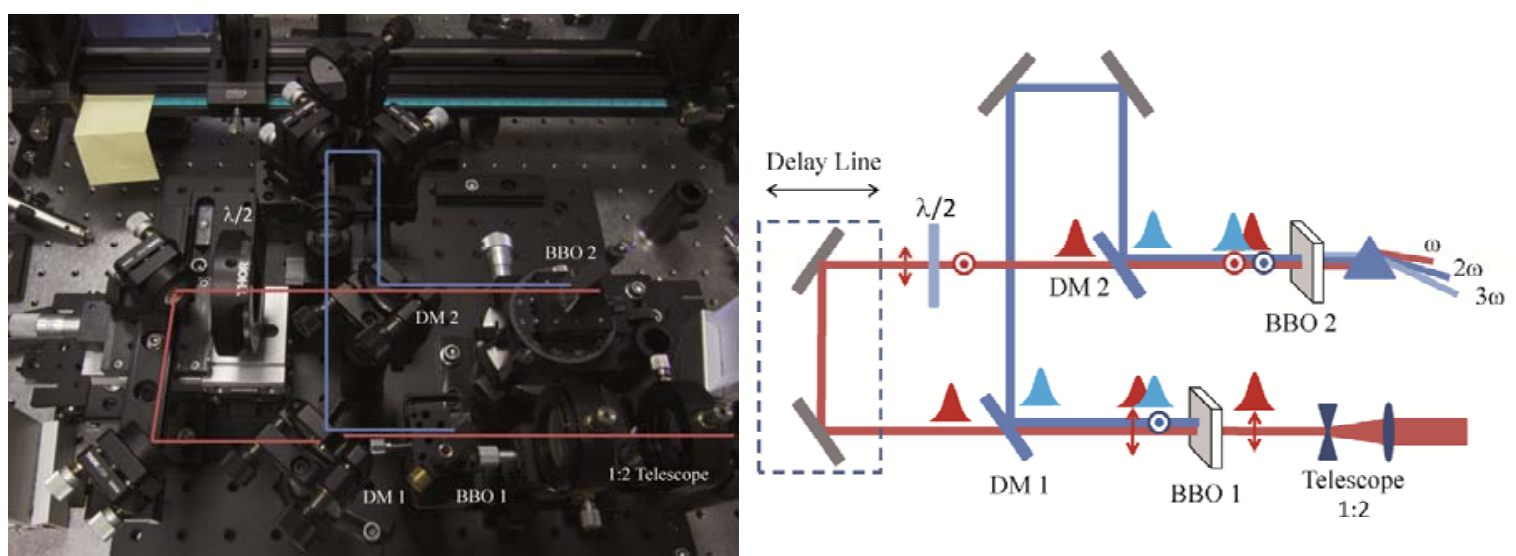

Figure 3.5. Photograph of the SHG/THG module in which the ray-tracing is shown to help in the visualization of the beams' paths (left). Sketch of the module where all the optical components are indicated (right). DM: dichroic mirror.

\footnotetext{
${ }^{10}$ A beam with spatial chirp has its different frequency components separated in space transverse to the propagation direction.
} 


\subsection{Design}

Concerning design, the first issue was to minimize the set-up in accordance with the foreseeable space. We needed it to be compact so that it could be carried easily from one optical table to another. Therefore, prior to implementation we focused our attention on the design. Several issues appeared:

- The first one was to compensate the paths that the second-harmonic and the fundamental pulses were going to travel. These two paths must have the same length so that the pulses can arrive together at the nonlinear crystal where the sum-frequency signal will be produced.

- Second, it was necessary to match the height of the different elements and to select the appropriate optical coatings. For example the mirrors must have good reflectivity in the blue, etc.

- The next issue was to choose the type of interaction geometry for the sum process. As is shown in the figure we chose a collinear interaction. This has the disadvantage that the pulses are not easily separated, but the advantages compensate the pitfalls, because in comparison with the noncollinear geometry the spatial and temporal overlapping of the pulses must be maximized, also maximizing the conversion efficiency.

- Finally, we introduced a delay line, where commercial systems use a compensation plate that compensates for the difference in the group velocities of the pulses prior to entering the sum-frequency crystal. The use of the delay line makes the module suitable for performing characterization measurements of second-harmonic pulses.

Apart from these more technical issues, it is also necessary to deal with the choice of the right crystal, in order to maximize -among other aspects- the conversion efficiency in the SHG. Accordingly, we shall analyze this matter in detail in the following section.

\subsubsection{Choosing the Proper Crystal}

There are different parameters that need to be considered in order to choose the right crystal for a given application. In the following section, we analyze the characteristics of $\mathrm{BBO}$, which is the nonlinear crystal used in these experiments, as well as in the following experiments performed in this thesis. This crystal is the one most used in this kind of application-OPA, SHG, SFG- with Ti:Sapphire lasers. Here we shall analyze the different properties that make this crystal the preferred one and at some point we shall compare its features with another popular one; namely, $\mathrm{KDP}\left(\mathrm{KH}_{2} \mathrm{PO}_{4}\right)$.

Physical robustness. On the one hand, it has a high damage threshold, a fluence of $13 \mathrm{~J} / \mathrm{cm}^{2}$

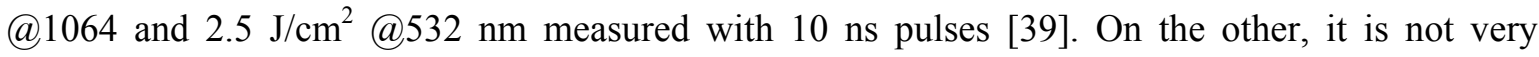
hygroscopic. This has the advantage that there is no need to work in a vacuum, without not much degradation [41] and that it can be used without a coating.

Range of transparency. This is a very important issue. Having a wide range of transparency spans the regions of the electromagnetic spectrum where a crystal can be used as a frequency doubler, provided that phase matching can be achieved. 
In Figure 3.6 we represent the transmission of both nonlinear crystals $-\mathrm{BBO}$ and KDP- after 1 $\mathrm{cm}$ of material. We see how the BBO transparency is expanded, covering the infrared region of the spectrum, which is not the case for the KDP. However, for this application in particular the SHG and THG of the Ti:Sapphire laser both crystals show the same performance. Nonetheless, the problem would appear for the optical amplification process, in which frequencies in the IR region are generated. If these frequencies are absorbed by the medium, amplification cannot occur. We shall see this in detail in Chapter 7, but we can understand it as obeying the coupled wave equations (2.74) introduced in the previous chapter, which describe how the three waves might interact at the same time.

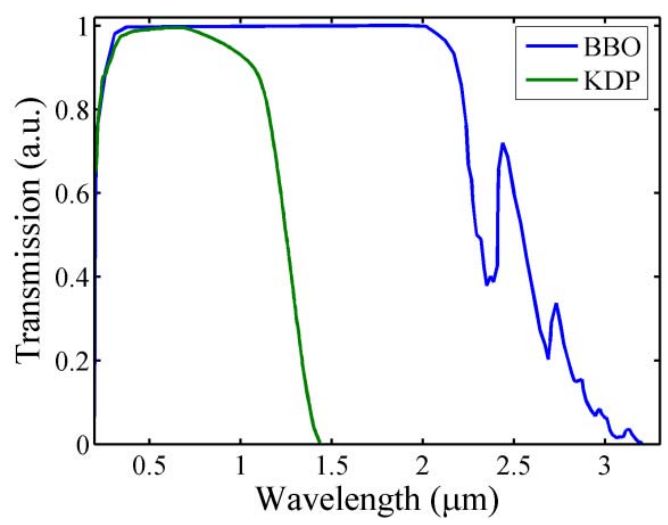

Figure 3.6. Optical transmission through $1 \mathrm{~cm}$ of material for the nonlinear crystals $B B O$ and KDP. Source SNLO [39].

Efficiency. To account for the conversion efficiency, we can refer to equation (2.61) in the previous chapter, where we obtained the efficiency of the SFG process under the undepleted pump approximation, and then particularize for the case of $\mathrm{SHG}^{11}$, obtaining:

$$
\eta=\frac{I_{2 \omega}(L)}{I_{\omega}(0)}=\frac{8 \omega^{2} d_{e f f}^{2} I_{\omega}(0)}{n_{2 \omega} n_{\omega}^{2} c^{2} \varepsilon_{0}} \mathrm{~L}^{2} \operatorname{sinc}^{2}\left(\frac{\Delta k L}{2}\right)
$$

According to this result, it would be logical to think that, when the phase-matching condition is met, efficiency would increase with increasing intensity and when the crystals are as long as possible. However, we have already explained that this is not so evident when working with femtosecond pulses. Even so, we can return to Eq. (3.16) to address the influence of the nonlinearity itself. This factor is not affected by the use of femtosecond pulses. Here, the nonlinearity is represented by $d_{\text {eff }}$, the so-called effective nonlinear optical coefficient.

In the table below we compare the values of this quantity for the BBO and the KDP, for the two nonlinear processes that we shall generate in the module, (SHG and THG by means of the SFG of the fundamental and the second harmonic). It may be seen that for both types of interaction the nonlinearity is higher ( $\sim 6$ times) in the case of BBO.

\footnotetext{
${ }^{11}$ To obtain this equation without solving a similar system (2.55)-(2.57) for SHG, one needs to consider $\mathrm{I}_{1}=\mathrm{I}_{2}=\mathrm{I}_{\omega}, \omega_{1}=\omega_{2}=\omega, n_{1}=n_{2}=n_{\omega}, n_{3}=n_{2 \omega}$ and $d_{e f f}(\mathrm{SHG})=2 d_{e f f}(\mathrm{SFG})$.
} 


\begin{tabular}{cc|ccc|}
\cline { 2 - 5 } & \multicolumn{2}{c}{$d_{\text {eff }}(\mathrm{pm} / \mathrm{V}) \mathrm{SHG}$} & \multicolumn{2}{c}{$d_{\text {eff }}(\mathrm{pm} / \mathrm{V}) \mathrm{THG}$} \\
\cline { 2 - 5 } & $\mathrm{BBO}$ & $\mathrm{KDP}$ & $\mathrm{BBO}$ & $\mathrm{KDP}$ \\
\hline Type I & 2 & 0.30 & 1.86 & 0.04 \\
Type II & 1.09 & 0.25 & 0.06 & - \\
\hline
\end{tabular}

Table 3.1. Values of the nonlinear susceptibility for the nonlinear crystals $B B O$ and KDP for two nonlinear processes: $S H G$ and THG (by $S F G$ ) of the Ti:Sapphire laser. The data were extracted from the SNLO software [39].

Phase-matching bandwidth. In the previous chapter we explained how the phase mismatch influences the efficiency of the SHG and SFG processes; see Sect. 2.5.1. In that case, perfect phase matching was achieved with the proper orientation of the optical axis of the crystal with respect to the direction of propagation of the incident light. This was valid for a monochromatic wave, i.e. only one frequency component, the phase-matching condition being:

$$
\Delta k=2 k_{1}-k_{2}
$$

Nevertheless, what happens when the input is a not monochromatic wave, such as a femtosecond pulse? Of course, the orientation of the optical axis is unique and hence only one component of the field can be perfectly phase-matched and satisfy $\Delta k=0$. Consequently, the other components will undergo a mismatch, and as a result their efficiency in the conversion will be lower. Thus, $\Delta \mathrm{k}$ proves to be a function of wavelength, as we shall now see. If we apply Type I phase-matching conditions, where the fundamental is an ordinary wave and the second harmonic generated is an extraordinary one, condition (3.17) transforms to:

$$
\Delta k=2 \pi\left(\frac{2 n_{o}\left(\lambda_{1}\right)}{\lambda_{1}}-\frac{n_{e}\left(\lambda_{2}, \theta_{P M}\right)}{\lambda_{2}}\right),
$$

where $\theta_{P M}$ also depends on wavelength. However, we shall allow it to be the phase-matching angle for the central wavelength of our pulse. The phase-matching angle can be calculated from Eq. (2.73). To conclude, we take into account the energy relation $2 \omega_{1}=\omega_{2}$ or, similarly $\lambda_{2}=\lambda_{1} / 2$, and finally the phase-matching condition is transformed into the equation:

$$
\Delta k=\frac{4 \pi}{\lambda_{1}}\left(n_{o}\left(\lambda_{1}\right)-n_{e}\left(\lambda_{1} / 2, \theta_{P M}\right)\right)
$$

which is a function of wavelength, and hence the efficiency in the generation. We can plot the phase-mismatch factor, defined in the previous chapter:

$$
\operatorname{sinc}^{2}\left(\frac{\Delta k(\lambda) L}{2}\right)
$$

for different crystals and crystal sizes, $L$, see Figure 3.7. Note that apart from the dependence on crystal size the refraction index characteristics of the crystal are included in the phase-matching function. As expected, this function has its maximum at the central wavelength of the pulse, where $\Delta k=0$; in our case $\lambda_{\Delta k}=0.795 \mathrm{~nm}$. 

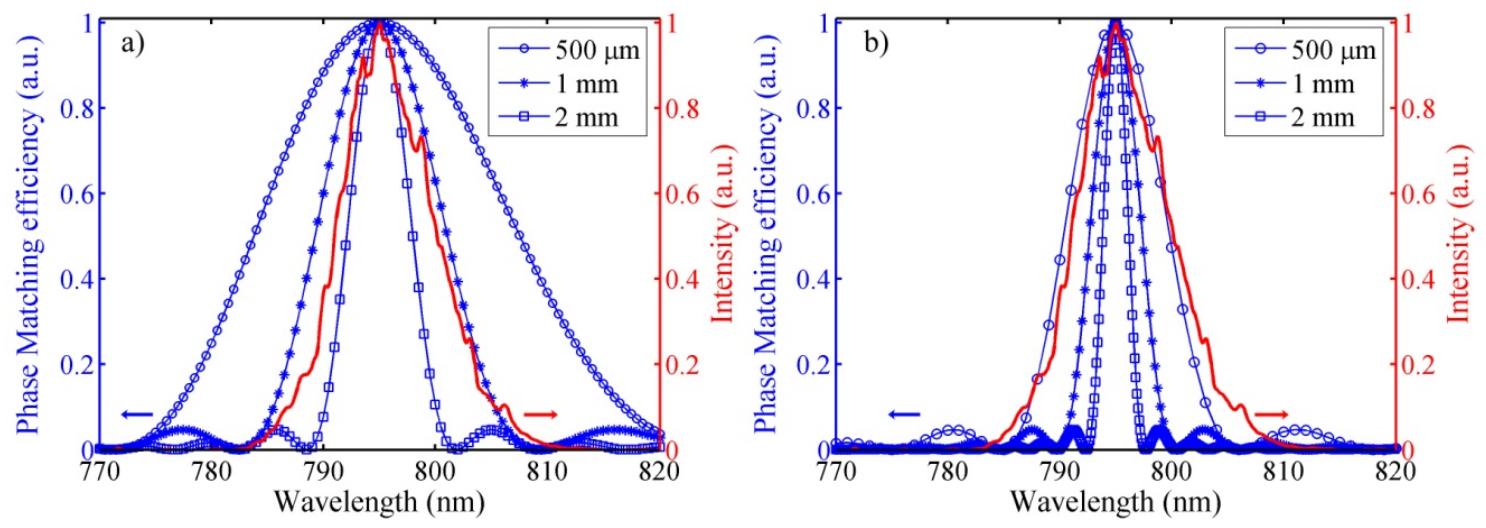

Figure 3.7. Phase-matching curves for two crystals: KDP-panel a) and BBO panel b) for different crystal thicknesses, indicated in the legend. In both cases a typical spectrum of the Ti:Sapphire laser ( $\sim 9 \mathrm{~nm}$ at intensity FWHM) is also plotted.

It may be seen how the trend is the same in both cases; as the crystal increases in thickness, the phase-matching curve becomes narrower. However, there are some differences between the two crystals. In the case of BBO, only the $0.5-\mathrm{mm}$ thick crystal matches the spectrum of the laser. In the other two cases, some of the frequencies will not be doubled with a reasonably good conversion and in some cases efficiency drops to zero. Therefore, in terms of phase-matching bandwidth KDP shows better performance than BBO. Note how with a 1-mm thick crystal we match the curve of the laser.

At this point, other aspects need to be taken into account in order to make the decision about which crystal to choose, for example the potential conversion efficiency. A good question might be: which is more efficient: a $1 \mathrm{~mm} \mathrm{KDP}$ or a $0.5 \mathrm{~mm}$ BBO crystal? The answer to this question is in the nonlinearity $\mathrm{d}_{\text {eff; }}$, and this is what makes the difference. Looking at Table 3.1, and making use of Eq. (3.16), a KDP as thick as $3 \mathrm{~mm}$ is needed to obtain the same conversion efficiency as with a $0.5-\mathrm{mm}$ BBO. Therefore, to obtain the same conversion efficiency we would have to limit the converted spectrum in the case of KDP. This is undesirable when working with femtosecond pulses because if the spectrum becomes narrowed the temporal duration of the pulses will be increased, as we are about to see.

a)

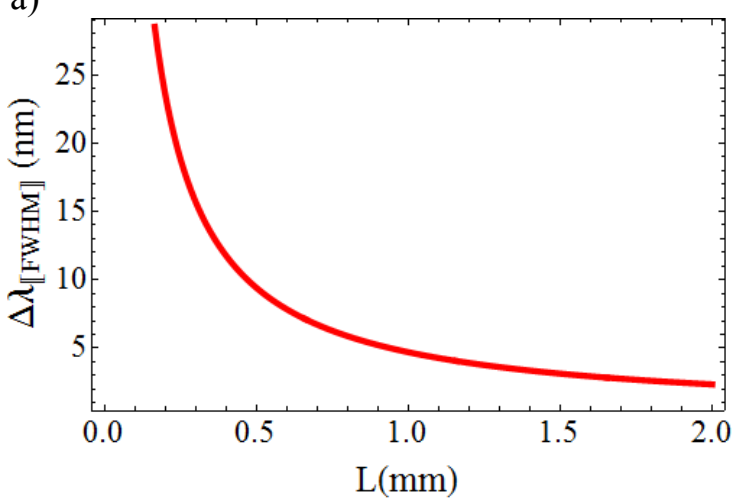

b)

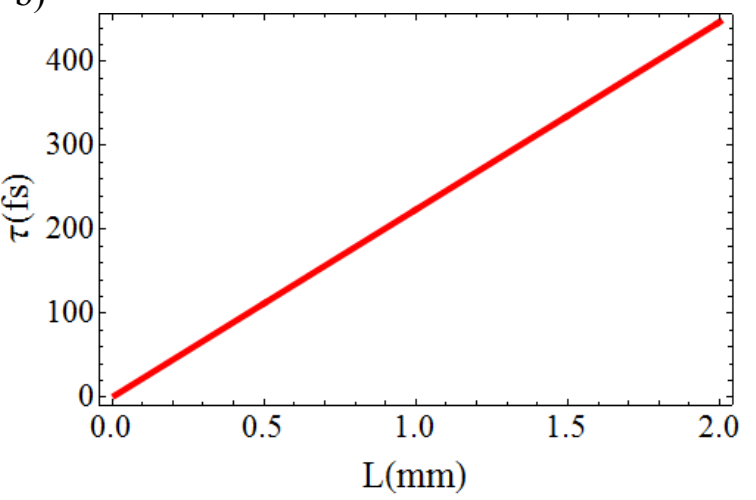

Figure 3.8. a) Phase-matching bandwidth at intensity FWHM for Type I SHG in BBO. b) Fourier-transform limit corresponding to an IR pulse $\lambda_{c}=795 \mathrm{~nm}$ with the bandwidth from a). 
By performing an expansion to first-order in (3.19), we can obtain a relation of phase-matching bandwidth at FWHM with crystal length [42]. We have plotted this in Figure 3.8. We see how the increase in crystal length decreases the phase-matching bandwidth. However, it is more practical to plot the Fourier-transform limit associated with this bandwidth, and also with crystal length. In other words, for fundamental pulses with time duration of $\tau$ we can determine the most suitable crystal size for doubling those pulses without limiting the IR spectra. For example, if we need to double $100 \mathrm{fs}$ pulses we would require a $500 \mu \mathrm{m}$ crystal

\subsection{Measurements and Results}

In this section we describe the different kinds of measurements that we have made with the module. The section is organized as follows: first we shall introduce the measurements related to the SHG, where only one nonlinear crystal is involved (BBO1 in Figure 3.5). Regarding the latter, we shall continue with the characterization of these pulses. In the last part of this section, we shall report the results obtained upon dealing with the THG by means of the SFG process.

\subsubsection{Second-Harmonic Conversion Efficiency}

Our first aim was to use the module for the simple but necessary task of characterizing the different crystals in terms of conversion efficiency. The first set of measurements was performed with a 1$\mathrm{mm}$ thick BBO sample. At the laboratory, the first step was to adjust, with the help of the spectrometer, the orientation of the crystal. When the crystal is properly oriented the signal is maximized in the spectrometer, with no modulation, and is centred at the correct wavelength. After this fine adjustment of the crystal, we started the efficiency measurements. Thus, we changed the input energy, with a half-wave plate and a linear polarizer. For each different input fundamental energy we measured the output second harmonic after two bounces in the dichroic mirrors. The energy of both pulses was measured with a commercial power meter (Spectra-Physics, Model 407A).

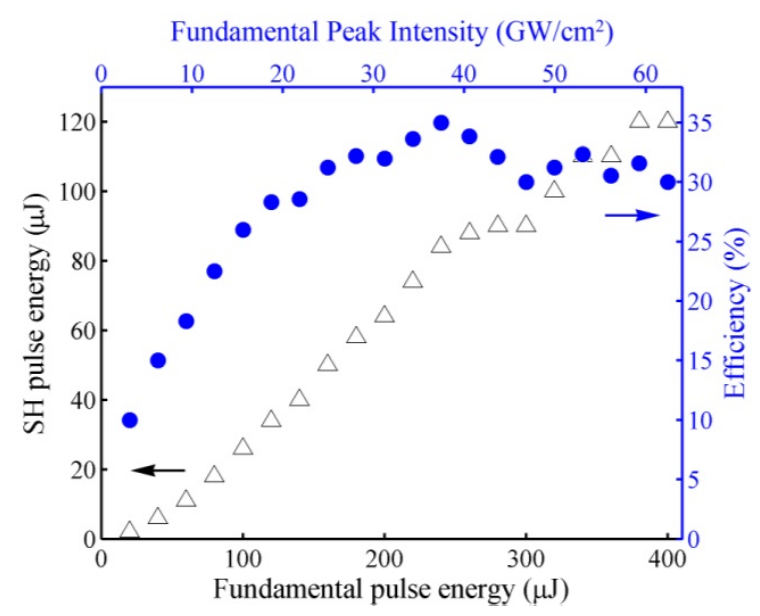

Figure 3.9. Representation of the second-harmonic pulse energy in terms of the fundamental's pulse energy and peak intensity-open triangles-along with a representation of the conversion efficiency-filled circles-for a 1-mm thick BBO crystal.

In the case of the $\mathrm{BBO}$ with a thickness of $1 \mathrm{~mm}$ the results are displayed in Figure 3.9 a), where we have represented the second-harmonic pulse energy in terms of the input energy and peak intensity, along with the conversion efficiency, defined as the second-harmonic energy divided by 
the input IR energy. Here, we are considering the external efficiency; i.e. we are not taking into account reflection losses due to the absence of antireflective coating. If we had considered the internal efficiencies, as done in the simulations, we would have expected higher values. In the lowenergy part of the curve, the trend is the expected one: the output second-harmonic pulse energy increases with the increase in the input fundamental. However, the conversion efficiency reaches a maximum, after which it decays and displays oscillatory behaviour. This fits in well with the simulations that we discussed in Sect. 3.3.1 and also with similar experiments made by the authors in reference [37].

We also measured the efficiency in another BBO sample, in this case a 0.5 -mm thick. The results are plotted in Figure 3.10 a). It may be seen that efficiency is lower at all the intensities measured. However, as the input energy is increased the difference becomes lower and does not follow the trend shown in Eq. (3.16), as occurred in the simulations.

From a practical point of view, the $1 \mathrm{~mm}$ BBO crystal is preferable, and with this we will gain more blue energy out of the crystal; in the application of materials micro-processing this is sufficient. However, in applications where it is important to maintain both the broadband spectra, and hence the time duration of the pulses, the thinner one would be preferable.

To illustrate this latter point, we also recorded the second-harmonic spectra obtained with both crystals. In the following figure it can be seen that the bandwidth is greater for the case of the thinner crystal, as expected from the phase-matching curves introduced previously. At intensity FWHM the bandwidth are $2.5 \mathrm{~nm}$ for the $0.5-\mathrm{mm}$ thick BBO and $1.9 \mathrm{~nm}$ for the other crystal. The corresponding Fourier-limited pulse time durations are 106 and 156 fs respectively.

a)

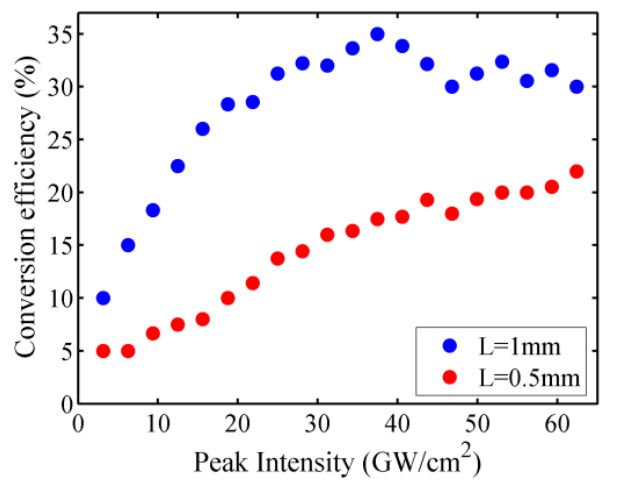

b)

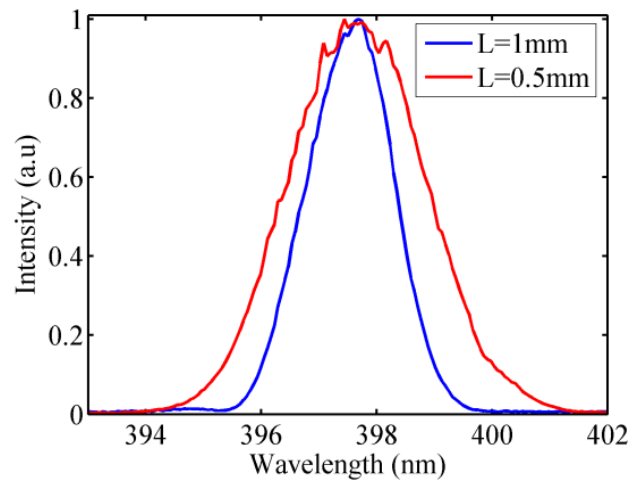

Figure 3.10. a) Optical conversion efficiency measured in two BBO samples of the thicknesses indicated in the legend. b) Spectra measured in the blue of the second harmonic generated with $\mathrm{BBO}$ with the same thicknesses.

\subsubsection{Second-Harmonic Characterization}

One problem emerged, associated with the generation of ultrashort pulses: their characterization. There are several methods for characterizing femtosecond pulses, the vast majority of them based on nonlinear optics and, in particular, many of them based on processes of frequency conversion, such as the SHG, the SFG or the DFG. In our case, with this module we are able to make both XFROG (Cross-Frequency Resolved Optical Gating) [43] and Cross-correlation measurements [44] to characterize the second-harmonic pulses generated in the first BBO. These types of characterization methods are based on having a test pulse, which have been characterized 
previously (usually with a similar device, FROG [45] or autocorrelator), after which a nonlinear process is generated involving the reference/test pulse and the one that needs to be characterized: in our case the second harmonic. Therefore, our test pulses, also called gate pulses, will be the IR fundamental pulses.

These two methods differ in the amount of information that we can obtain about the unknown pulse. While in the X-FROG, we can characterize the pulse completely, including intensity and phase, with the cross-correlation we can only estimate their time duration. Moreover, detection of the generated signal is also different: in the case of the cross-correlation, a power meter or a photodiode is sufficient to obtain the trace, but in the case of the X-FROG a spectrometer is needed to obtain the trace, which relates wavelengths with delay.

One might wonder why we used a cross-correlation method instead of using the standard autocorrelation or FROG. We did this because the traces of these violet-blue pulses $(400 \mathrm{~nm})$ cannot be obtained, owing to a lack of nonlinear crystals where SHG can be phase-matched for this wavelength range. In BBO, for instance, Type-I phase-matching for SHG is only possible down to $410 \mathrm{~nm}$. The limit in the case of Type II phase-matching is at even longer wavelengths. On top of this, in our case the problem could also stem from the detection of the resulting signal at $200 \mathrm{~nm}$, because this wavelength cannot be detected by any of the spectrometers available at the laboratory. Thus, the easiest solution for us was to record the cross-correlation and X-FROG traces between the second-harmonic and the fundamental pulses using the phenomenon of SFG as the nonlinear process.

In both methods, the two pulses arrive at the nonlinear crystal with different relative delays, which in our case could be changed manually with the delay line indicated in Figure 3.5. The thirdharmonic power is measured in each delay position, obtaining a power maximum when the maximum of the pulses overlaps in the crystal and drops to zero when the pulses are not temporally overlapped.

As mentioned in this chapter, the main idea is to have available in the laboratory new sources in the blue and the UV wavelength range. It is good that our experimental arrangement also affords the possibility characterizing the second-harmonic pulses emerging from the first BBO crystal. Here, we shall present the characterization measurements of the second harmonic generated in the two BBO crystals used in the previous section.

An important issue is to choose the appropriate crystal for SFG. On the one hand, it is necessary to calculate the phase-matching angle, which in principle will be different from the secondharmonic angle. On the other hand, the crystal chosen for the cross-correlation and the X-FROG measurements needs to be thin enough to prevent the dispersion effects described above (GVM in particular).

Regarding the cut angle, when describing the set-up we have already mentioned that the crystal cut angle required to perform the SFG was $44.7^{\circ}$. This angle can be calculated through the relation given in Table 2.1, in the previous chapter, for a Type I interaction in a uniaxial negative crystal:

$$
n_{e}\left(\omega_{3}, \theta\right) \omega_{3}=n_{o}\left(\omega_{1}\right) \omega_{1}+n_{o}\left(\omega_{2}\right) \omega_{2}
$$

This reduces to the following expression, with $\omega_{1}=\omega, \omega_{2}=2 \omega$ and $\omega_{3}=3 \omega$ 


$$
3 n_{e}(3 \omega, \theta)=n_{o}(\omega)+2 n_{o}(2 \omega)
$$

As we did for the SHG, it is possible to calculate the phase-matching angle using the phasematching condition (3.21) and Eq. (2.71).

Regarding crystal thickness, we chose a $100 \mu \mathrm{m}$-thick BBO to meet the requirements imposed mainly by the GVM. In this kind of measurement, the differences in the group velocities of the interacting pulses result in an effect called time smearing, where the cross-correlation trace is broadened artificially. Consequently, an unreal higher value of the pulse time duration is obtained.

Time smearing is the effect produced when the crystal thickness is large enough to cause a significant delay between the fundamental and second-harmonic pulses as they propagate through the crystal. In this case, the gate pulse does not probe a single "temporal" slice of the pulse to be measured, but instead a longer time interval. This leads to an artificially broadened X-Correlation curve, because the third-harmonic signal could be generated even though the fundamental and second-harmonic pulses do not overlap at the entrance of the crystal.

Therefore, if the pulses travel through the nonlinear crystal with length $L$ the product

$$
G V M \times L \ll \tau_{p}
$$

must be less than the time duration of the shorter pulse, $\tau_{p}$, to ensure that the relative delay between the incident pulses keeps nearly constant along the interaction. In this particular case, this is not very demanding because pulses with time duration of $120 \mathrm{fs}$, in $100 \mu \mathrm{m}$ (the chosen length of the crystal) will separate by only 20 fs.

We first report the measurements of the X-FROG for both second harmonic generated in a 0.5 $\mathrm{mm} \mathrm{BBO}$ and in a $1 \mathrm{~mm}$ BBO crystal. In both cases the input fundamental energy was $20 \mu \mathrm{J}$. The delay line allowed us to change the relative delay between the pulses, and with a spectrometer we recorded the third-harmonic signal centred in the UV at $265 \mathrm{~nm}$. With the spectra measured, we created the experimental traces shown below, in which the colour scale represents the normalized intensity of the third-harmonic signal. The zero delay is chosen in the maximum of both traces because it corresponds to the situation in which the peaks of the pulses arrive together at the crystal.
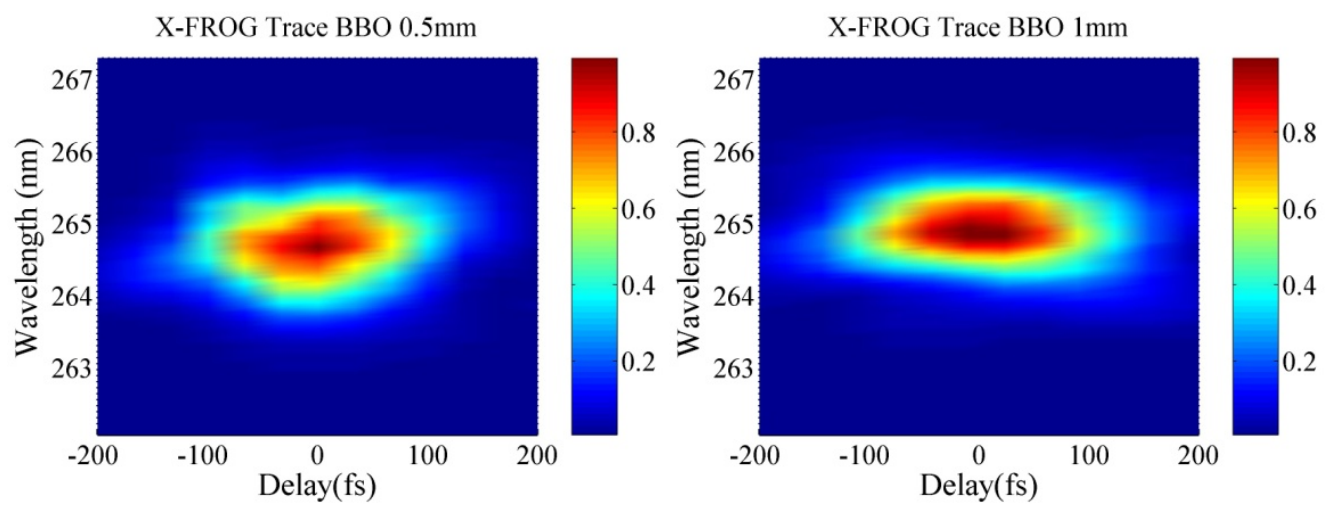

Figure 3.11. Experimental X-FROG traces from the characterization of second-harmonic pulses generated in $B B O$ with the thicknesses indicated at the top of each figure. 
Apart from the difference in the time duration of the two traces, being slightly longer in the case of the thicker crystal, as expected from the simulations made in Sect 3.2.1, it may be seen that the spectra are narrower in this case. This is a consequence of the reduction in the spectral bandwidth of the second harmonic generated with this crystal, as we show experimentally in Figure $3.10 \mathrm{~b}$ ).

With a commercial retrieval algorithm (FROG 3, Femtosoft Technologies [46]) we reconstructed the second-harmonic pulses for the case of the thin crystal. The information that the program offers is, on one hand, a retrieved trace that must look very similar to the original experimental trace recorded and, on the other, is directly related to the pulses, such as the pulse intensity and temporal phase, and the spectral intensity and its spectral phase. An example of a data set is depicted in Figure 3.12.

Prior to introducing the data into the program we needed to characterize the test pulses, (fundamental). At our laboratory this task can be undertaken by using two different methods: Grenouille -a commercial FROG [47]- and the single-shot autocorrelator. The fundamental pulses have duration of $\sim 130 \mathrm{fs}$, measured with the Grenouille.
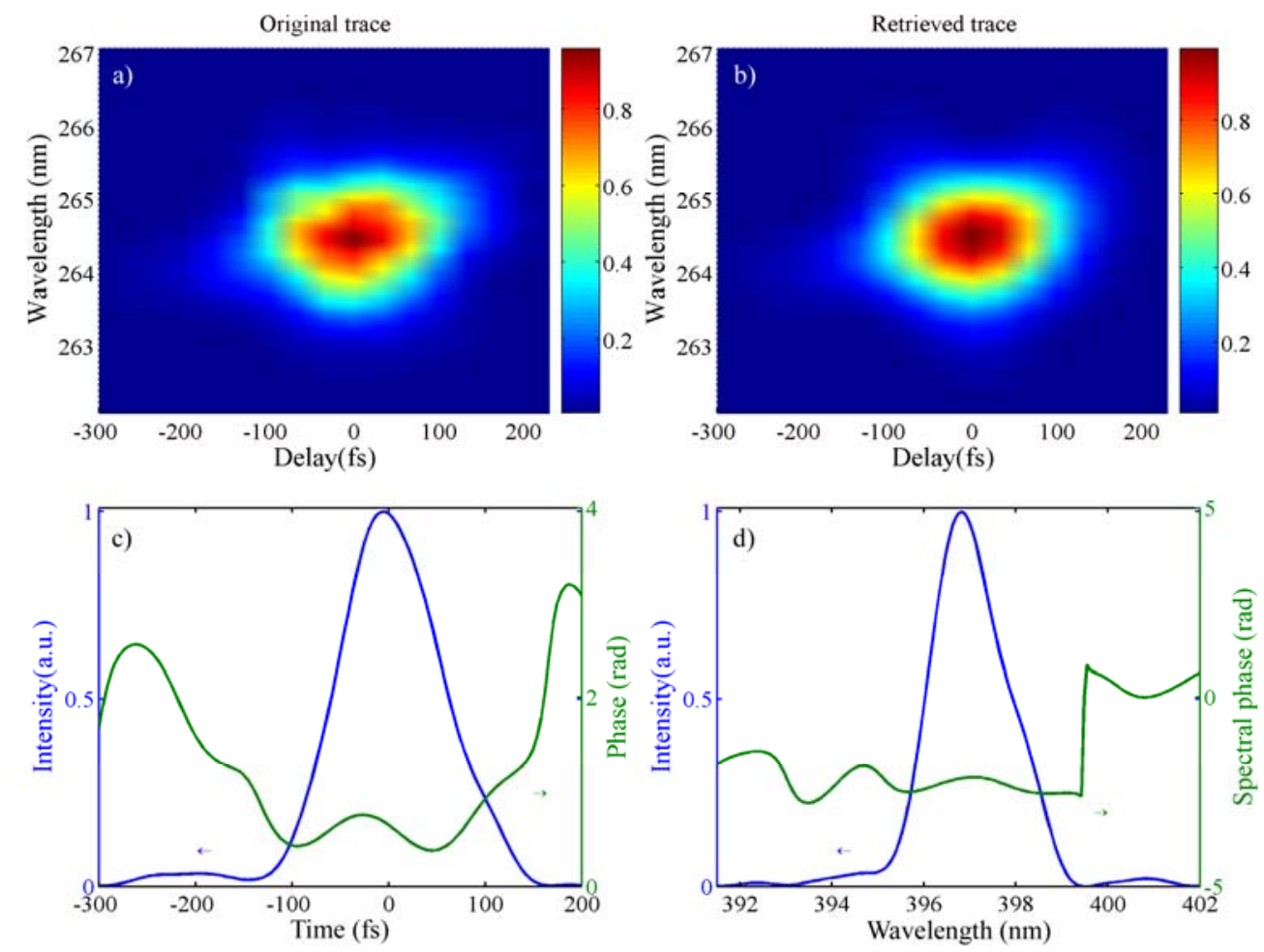

Figure 3.12. a) Experimental and b) retrieved X-FROG traces. The colour map represents intensity in normalized arbitrary units. c) Reconstructed pulse in time along with the temporal phase -green line-.d) Retrieved spectral intensity along with the spectral phase -green line-

After retrieval, the duration of the second-harmonic pulses at intensity FWHM is $\sim 125 \mathrm{fs}$, with a time-bandwidth product of 0.45 . The best error in the retrieval was of the $0.5 \%$. This is a very good result, confirming that the short duration of the fundamental pulses is preserved and that the second-harmonic pulses are not chirped. 
Obtaining the cross-correlation traces was easy once we had obtained those generated with XFROG. The cross-correlation is the integration in the wavelength of the previous traces (Figure 3.11). Experimentally, this can be achieved using a photodiode or a power meter instead of a spectrometer to record the power in the ultraviolet signal in each relative delay. The intensity of the cross-correlation trace is proportional to the convolution of the intensity of the two interacting pulses:

$$
I_{C C}(\tau) \propto \int_{-\infty}^{\infty} d t A_{b l u e}^{2} \times A_{\text {red }}^{2}(t-\tau)
$$

As mentioned, this measure provides less information than X-FROG. From the convolution, Eq. (3.24), one obtains a relation that gives, in an initial approximation, the time duration of the secondharmonic pulses [48]:

$$
\tau_{C C}=\left(\tau_{\text {red }}^{p}+\tau_{\text {blue }}^{p}\right)^{1 / p}
$$

with $\tau_{c c}, \tau_{\text {red }}$ and $\tau_{\text {blue }}$ being the time duration at intensity FWHM of the cross-correlation, the IR and the blue pulses, respectively, and with $p=2$ for Gaussian pulses and with $p=1.6151$ for $\operatorname{sech}^{2}$ pulses.

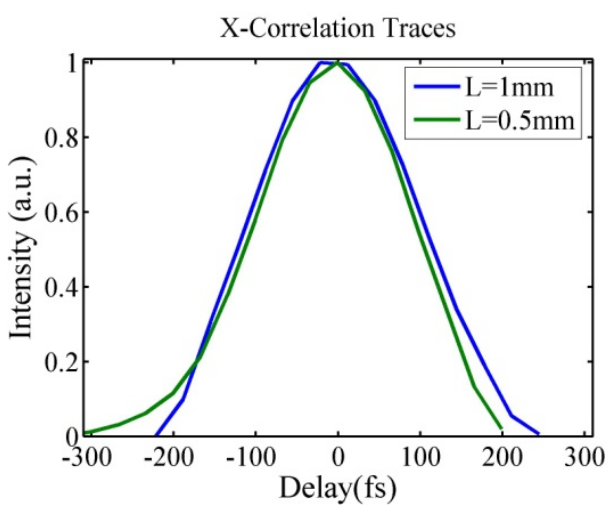

Figure 3.13. X-Correlation traces measured to characterize the second-harmonic pulses generated in two BBO crystals with the thicknesses indicated in the legend.

The results obtained upon applying equation (3.25) are summarized in the following table. We see how the pulse duration is greater in this calculation than that retrieved in the algorithm. This can be attributed to the fact that a given shape, $\left(\operatorname{sech}^{2}\right)$, of the pulse intensity is assumed in the calculation, and -as seen in the retrieved intensity Figure $3.12 \mathrm{c}$ )- the shape is not symmetric.

\begin{tabular}{c|c|c|c|c}
\cline { 2 - 5 } & \multicolumn{2}{c}{ BBO $0.5 \mathrm{~mm}$} & \multicolumn{2}{c}{ BBO $1 \mathrm{~mm}$} \\
\hline Input IR & $\tau_{\mathrm{CC}}(\mathrm{fs})$ & $\tau_{\text {blue }}(\mathrm{fs})$ & $\tau_{\mathrm{CC}}(\mathrm{fs})$ & $\tau_{\text {blue }}(\mathrm{fs})$ \\
\hline Energy $(\mu \mathrm{J})$ & 220 & 156 & 239 & 180 \\
\hline 20 & & 156 &
\end{tabular}

Table 3.2. Summary of the temporal duration of the cross-correlation and the blue pulses generated with the 0.5-mm-and the 1-mm thick BBO crystals.

As seen, the cross-correlation provides a greater temporal duration of the pulses generated in the thicker crystal. This is exactly what we expected, owing to a reduction in the converted spectra, as shown in Figure 3.10 b). Moreover, since the crystal is thick the blue pulses could also be chirped 
by dispersion. It would be interesting to perform a retrieval with the X-FROG algorithm, as we did for the other crystal. However, our experimental data are not good and the algorithm does not converge to anything reasonable. We attribute this to the lack of data at the edges of the trace when the signal drops to zero (See Figure 3.11, right panel).

\subsection{Generation of the Third Harmonic by SFG}

The last part of the present chapter will be devoted to the generation of third harmonic. In this case, to obtain more efficiency than for the case of the characterization we changed the BBO 2 for a 1$\mathrm{mm}$ thick one $\left(\theta=44.7^{\circ}, \phi=0^{\circ}, 14 \times 14 \times 1 \mathrm{~mm}^{3}\right)$. BBO 1 corresponds to the 1 -mm thick sample. See Figure $3.5 \mathrm{~b}$ ).

In Figure 3.14 a) we have plotted the third-harmonic energy as a function of the fundamental pulse energy entering BBO 1. We see the same trend in efficiency (calculated as the third-harmonic energy divided by the IR energy at the entrance of the module), which reaches a maximum and then displays oscillating behaviour.

In Figure $3.14 \mathrm{~b}$ ) we again plot the energy of third harmonic but in terms of the product of the fundamental pulse by the second-harmonic energy, both measured at the entrance of BBO 2. This representation was done to show how in the low-energy part the efficiency is linear with this product, as predicted from Eq. (2.61).

a)

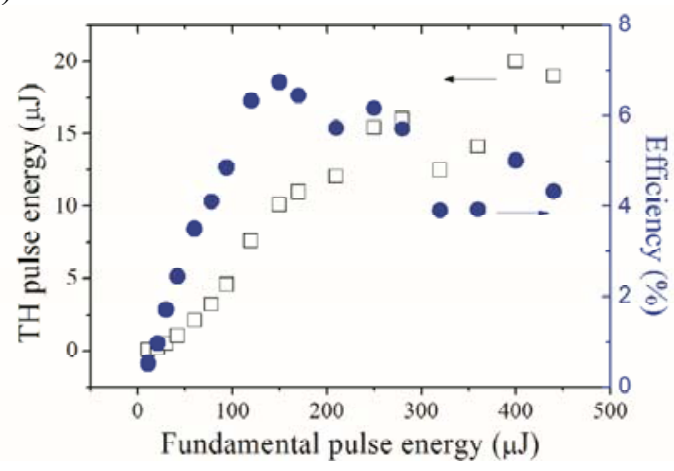

b)

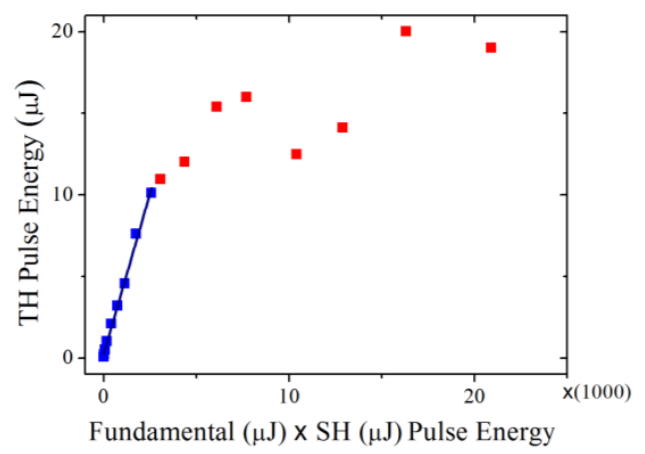

Figure 3.14. a) Third Harmonic (TH) pulse energy as a function of the fundamental pulse energy in BBO 1 along with the efficiency in the generation of the third harmonic. b) Thirdharmonic pulse energy as a function of the product of the fundamental by Second Harmonic (SH), both measured at the entrance of BBO 2. In this set of measurements a 1:3 telescope was used instead of the 1:2 telescope in Figure 3.5 b).

The oscillatory behaviour, seen above certain fundamental energy in the previous figures, is linked with the effect of efficiency saturation observed in the generated second-harmonic signal, (see Figure 3.9).

In this case the presence of other nonlinearities, (self-phase modulation, non linear absorption) due to the high intensity level is what degrades the SFG efficiency [37]. The oscillations are more evident in this case because we were reducing the beam with a 1:3 telescope, and with the smaller beam size, other spatial effects like hot-spots can also help to these higher order nonlinear effects to appear. 


\subsection{Conclusions}

We have designed and built a SHG/THG module adapted to the available laser source at the microprocessing laboratory. The availability of a customized device offers more possibilities of adaptability rather than commercial systems. For example, shorter pulses could be efficiently doubled with this module simply by changing the first nonlinear crystal.

Additionally, the set-up also offers the possibility of characterizing the second-harmonic pulses generated. For some applications, it is important to have a measure of the time duration of the interacting pulses.

We have also characterized the nonlinear crystals used in the module in terms of conversion efficiency in the second harmonic, and even in the third harmonic. In our opinion, it is important to have an estimation of the available energy in the UV wavelength range in the laboratory, above all when the pulses generated are to be applied in other experiments.

The module has been applied in material micro-processing with some interesting results which will be presented in the Chapter 8 . 


\section{Chapter 4}

\section{Third Harmonic Generation in a Micro-Structured BBO Crystal}

\subsection{Introduction}

In this chapter we shall focus on the generation of the third harmonic of the Ti:Sapphire laser in a noncollinear configuration. We shall explain how recording a 1D relief diffraction grating on the surface of a BBO crystal, by direct irradiation with femtosecond pulses, allowed us to generate the sum frequency of the fundamental and the second-harmonic pulses noncollinearly. The grating spacing was such that effective group-velocity matching, between the two incident pulses, was achieved. This opened the possibility of applying this customized device to intensity crosscorrelation measurements. As a consequence of the group-velocity compensation, the constraint of using a very thin crystal in this kind of measurement could be alleviated. Therefore, the aim of the work to be presented in this chapter was to exploit the capabilities of a customized photonic device with an application in the characterization of femtosecond pulses.

The chapter is organized as follows: in Sect. 4.2, we shall briefly introduce the materials microprocessing laboratory operating in Salamanca, where the grating was recorded. Then, in Sect. 4.3 we shall give the details of the recording procedure, and in Sect. 4.4, we shall describe the experimental set-up for the generation of third harmonic in the micro-structured crystal. The next section is devoted to describing the different processes taking place inside the crystal. In Sect. 4.5 
we conclude, reporting the application of the device built to intensity cross-correlation measurements.

\subsection{The Materials Micro-Processing Laboratory}

As already mentioned in the introduction of this thesis (Chapter 1), materials micro- and nanoprocessing with ultrashort pulses is one of the most widespread applications of the femtosecond laser systems; a field in continuous development that hopefully could soon also be implemented in some industrial procedures.

When intense femtosecond laser pulses are focused on the surface of a material (being either a dielectric, conductor or semiconductor), a process called ultrafast ablation is produced [19], which results in the ejection of material from the focal spot zone. By carefully controlling the focusing and irradiation parameters (number of pulses and fluence), the layer where the material has been removed can range from a few nanometres to several microns in thickness. Materials microprocessing can be also performed with longer laser pulses but femtosecond pulses have several advantages with respect to these [24]. For example, the material in the surroundings of the ablation zone remains barely unaltered, due to the minimization of thermal and mechanical collateral effects. Thus, the processing can be performed without significant damage to the substrate.

Moreover, femtosecond lasers can be focused inside transparent materials, such as glasses or crystals, and can modify their optical and structural properties at sub-micrometric scale. This possibility has resulted in a revolution in the design and fabrication of integrated optical circuits, since it became possible to write all types of structure inside these materials with the laser, such as waveguides [49], which confine and guide the light, as well as many kinds of three-dimensional photonic devices (diffractive elements, photonic crystals). For this kind of application, it is important to have available laser systems with a high repetition rate (1 $\mathrm{kHz}$ or more), such as the one at the laboratory in Salamanca. A high repetition rate is important because otherwise the processing time would be extremely long.

In Figure 4.1 the experimental arrangement used for making the gratings is shown. This system is part of the laboratory of Microprocesado de Materiales con Láser of the Universidad of Salamanca, where other complimentary systems are available, such as a trepanning-optics system, which allows holes to be made with trepanning and helical drilling techniques. With the latter system, it is possible to process thicker samples than with the conventional technique [50]. A set-up optimized for performing Laser Induced Breakdown Spectroscopy (LIBS) [51] is also available. This technique allows us to characterize the ablation products that can be used to control the ablation process spectroscopically, making the removal of material more selective. For all these systems, the laser beam comes from the adjacent laboratory through a window made in the wall. An electronic shutter is activated from the computer to control the number of pulses inciding on the sample. Then, different beamsplitters allow the beam to travel to one set-up or the other. We shall describe only the set-up used for recording the gratings, shown in Figure 4.1. After the shutter, a system formed by a motorized half-wave plate and a linear polarizer helps to adjust the desired pulse energy. The next step is to redirect the beam through the inverse U-shaped structure, with the help of three dielectric mirrors with good reflectivity in both the IR and UV radiation ranges. At the end of the structure, the focusing element is located, which focuses the laser directly into the sample; this can be either a microscope objective or a lens, depending on the required pattern size for each application in particular. The micro-patterning of the sample is usually made by 
transversal writing, i.e. with the incident beam focused perpendicular to the patterned surface. In the middle of the last column is the imaging system, formed by a cube beamsplitter and a CCD camera with an objective. The sample is illuminated with a conventional white-light lamp, and the image is formed in the camera. To focus the beam on the sample's surface, a fraction of the incident laser is left to propagate throughout the system and the spot at the surface can also be imaged with the camera. Then, the sample is moved along the direction of propagation ( $\mathrm{z}$ axis) to a position where the spot size in the camera is the smallest. The imaging system is essential to guarantee the correct positioning of the sample with respect to the beam and to perform fine adjustments of the focal length. A motorized XYZ positioning stage (with accuracy below $0.5 \mu \mathrm{m}$ ) and a rotation stage helps in performing the fine setting of the sample. The shutter and computer-controlled stages make it possible to generate any arbitrary pattern on the surface of the sample, such as, for example, the one displayed in Figure 4.2 a). Finally, an extractor system is used to remove all the ablation products that may be harmful to health.

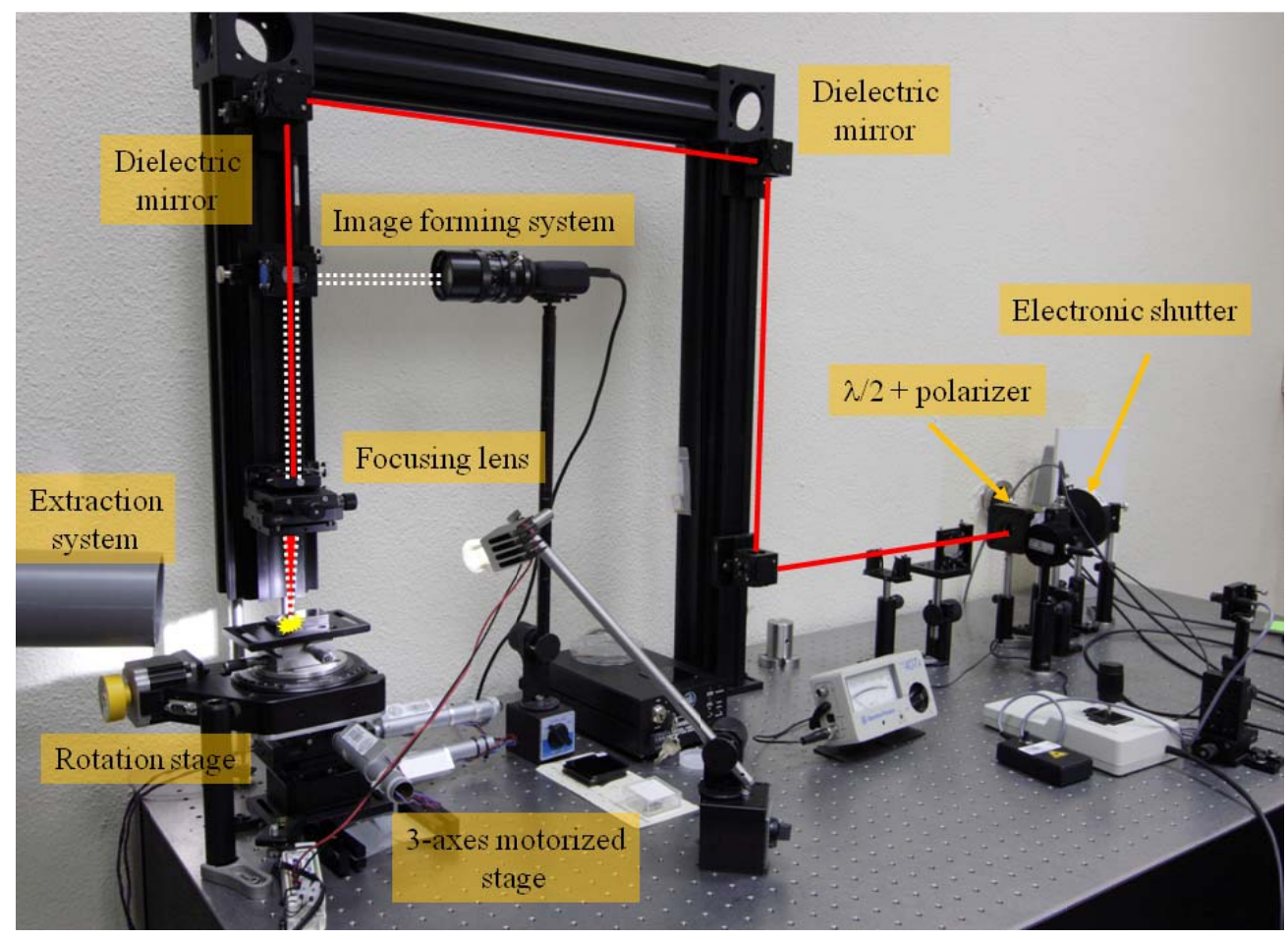

Figure 4.1. Picture of an experimental set up in the laboratory of Microprocesado de Materiales con Láser of the Universidad de Salamanca. The red lines represent the optical path for the IR or UV beams, and the white lines represent the optical path of the light collected in the camera from the sample, which helps to make the positioning. (See the text for further detail).

For the particular application of micro-structuring the BBO sample, all processing was performed in air under normal conditions of temperature and pressure. For other applications, controlled irradiation atmosphere may be required; for instance, for the generation of nano-particles in colloidal suspensions a surfactant is needed, typically deionized water [52].

As mentioned, femtosecond infrared laser pulses have been successfully used in the highprecision micro-structuring of transparent crystals and glasses. The application of this technique to the inscription of diffractive elements has been investigated exhaustively. Both internal [53] and 
relief 1D gratings [54] have been manufactured with high diffraction efficiency. In the latter case, a micro-structured nonlinear crystal was used for the generation of the noncollinear second harmonic among the diffraction orders. Also, 2D internal structures were recorded in KDP, obtaining a similar result, although greater control over the emerging beams was achieved in this case [55]. In both a 1D and a 2D structure, manufactured in Nd-doped crystals, laser action was also demonstrated [56].

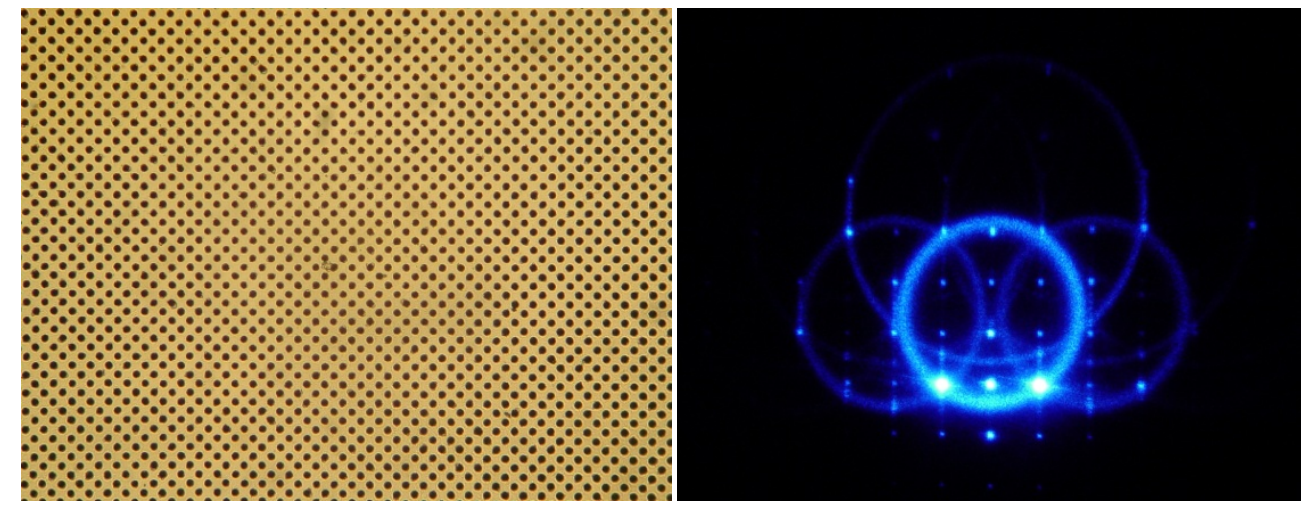

Figure 4.2. a) Optical microscope image of a $2 D$ pattern recorded on the surface of a KDP nonlinear crystal. b) Noncollinear second-harmonic beams projected on a screen. The image was taken from reference [55] with the permission of the authors.

\subsection{The Micro-Structured Crystal and the Grating Characterization}

Once again, BBO was the nonlinear crystal chosen for these experiments, for the same reasons adduced in Chapter 3 [41]. As said, for this experiment we needed to record a grating on a BBO sample to generate the third harmonic of the fundamental at normal incidence. As was done in the previous chapter, we generated the signal at $266 \mathrm{~nm}$ through the SFG process (of the fundamental $(795 \mathrm{~nm})$ and its second harmonic $(397.5 \mathrm{~nm}))$. Therefore, we used a crystal with cut angles for Type I phase matching at normal incidence: $\theta=44.7^{\circ}$ and $\phi=90^{\circ}$, with a size of $14 \times 14 \times 1 \mathrm{~mm}^{3}$. We chose a Type I interaction, where the input beams have the same polarization (ordinary) and the grating grooves were chosen to be perpendicular to the polarization of the beams.

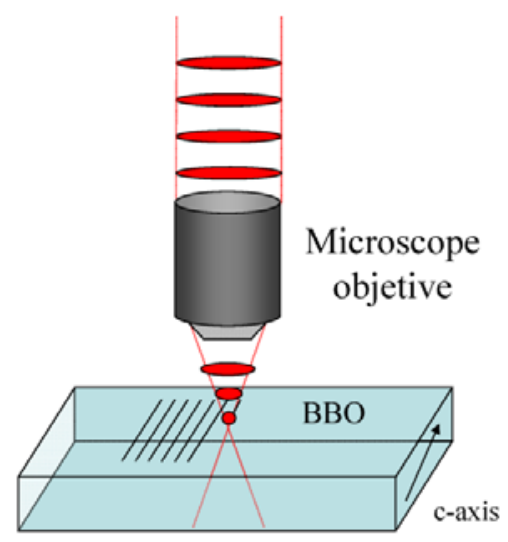

Figure 4.3. Sketch representing the writing procedure. It is important to note the relative orientation of the grating stripes, determining the diffraction plane, with respect to the optical axis (c-axis). 
In order to obtain very small grooves, a 50x microscope objective (EPI PLAN L, Leica) was used to focus the beam onto the crystal's surface. With this objective, the theoretical beam diameter at focus was $\sim 0.8 \mu \mathrm{m}$ (at $1 / \mathrm{e}^{2}$ intensity criterion). While the sample was irradiated with the laser, it was scanned along one axis at constant velocity, always keeping the focus on the surface. At this point several parameters were studied to find the minimum groove size. In particular, we tested different velocities and pulse energies. Finally, the parameters chosen were: $300 \mathrm{~nJ}$ as the energy per pulse, measured immediately in front of the objective, and $57 \mu \mathrm{m} / \mathrm{s}$ as the scanning velocity. This latter quantity was chosen as a compromise between the quality of the gratings and the processing time, which in this case was of approximately 9 hours for the desired size of the grating $\left(2.5 \times 2.5 \mathrm{~mm}^{2}\right)$. Another important parameter was the groove spacing; previous calculations were made to find the correct value, which proved to be $3.5 \mu \mathrm{m}$ (we shall see this in detail in Sect. 4.5). This value is at the limit of the long-range precision of the motorized stage.

a)

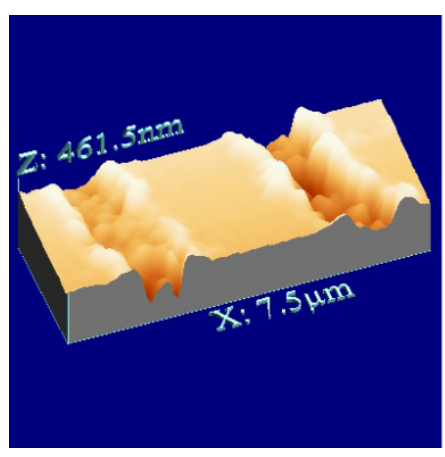

b)

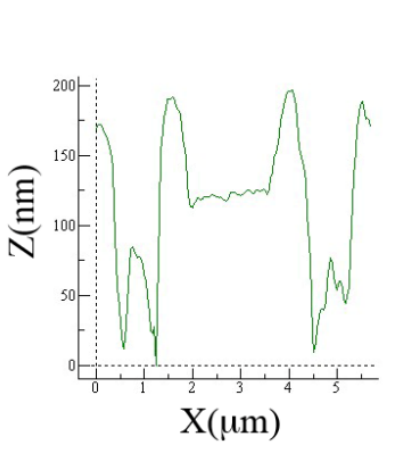

c)

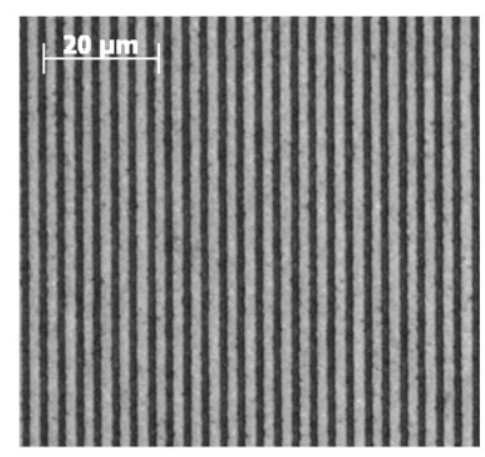

Figure 4.4. a) Topographic image of part of the grating from an AFM taken in contact mode. b) Profile from a) where the groove size and depth can be assessed. c) Optical microscope image of the grating. The excellent work done by J. A. Pérez to obtain the AFM images is acknowledged.

The grating was analysed with both a light and an Atomic Force Microscope (AFM). From the measurements in the AFM (see Figure 4.4 a) and b)) we were able extract a profile where we could approximately determine the groove depth, which turned out to be around 150-200 $\mathrm{nm}$. Prior to the AFM analysis, the light microscopy also revealed a groove width of $\sim 1 \mu \mathrm{m}$. An image of part of the grating taken in reflection mode (bright field) can be seen in Figure $4.4 \mathrm{c}$ ).

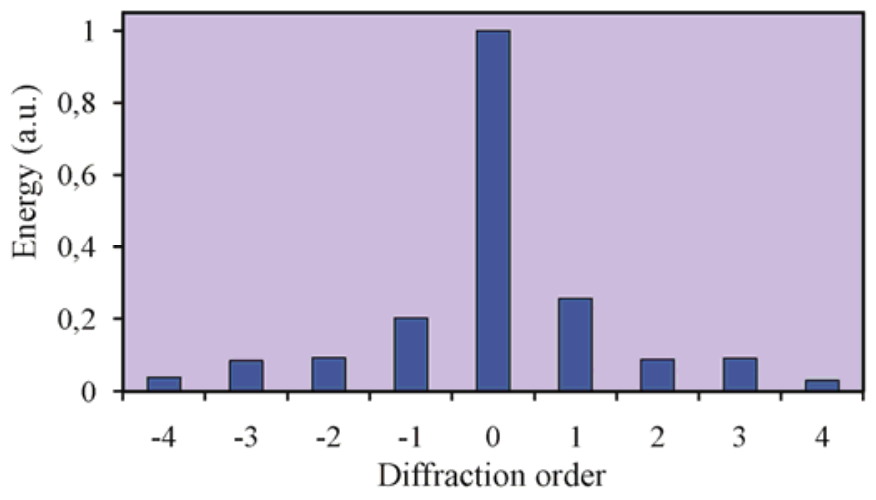

Figure 4.5. Normalized energy of the diffraction orders when irradiating the grating with an $\mathrm{He}-\mathrm{Ne}$ laser. 
Before going further, we characterized the grating in terms of diffraction efficiency (see Figure 4.5). We measured the power travelling in each diffracted order with a power meter. The efficiency was normalized to the power diffracted along the first order. We used a low-power He-Ne laser $(600 \mu \mathrm{m}$ beam size) for these measurements. The bar chart indicates that most of the energy travels along the zero-order of diffraction. The total energy travelling in the transmitted diffraction orders amounts to $49 \%$ of the incident.

\subsection{Experimental Set-Up for SFG and Results}

For the SFG we arranged a set-up (see Figure 4.6) similar to the one described in the previous chapter for the SHG/THG module. In this case the system was not portable and the beams' paths were longer than in that case. On the one hand, this hindered the system alignment and, on the other hand, it hampered the adjustment of the beams' paths so that the pulses (second harmonic and fundamental) would be temporally overlapped in the nonlinear crystal used for SFG.

Before this experimental set-up, the energy of the Ti:Sapphire laser was split with a $50 / 50$ beamsplitter (not included in the figure). One half of the energy travelled to the micro-processing laboratory described before and the other half was available for this experiment.

First, we generated the second harmonic of the fundamental pulses in a BBO crystal (BBO 1 in Figure 4.6) cut for Type I phase matching at normal incidence $\left(\theta=29.4^{\circ}, \phi=90\right)$ with a size of $14 \times 14 \times 0.5 \mathrm{~mm}^{3}$. As explained in the previous chapter, this crystal thickness is the most suitable for the SHG of our laser system, because, on the one hand, its phase-matching efficiency matched the bandwidth of the fundamental pulses and, on the other, it ensured no temporal lengthening of the second-harmonic pulses generated.

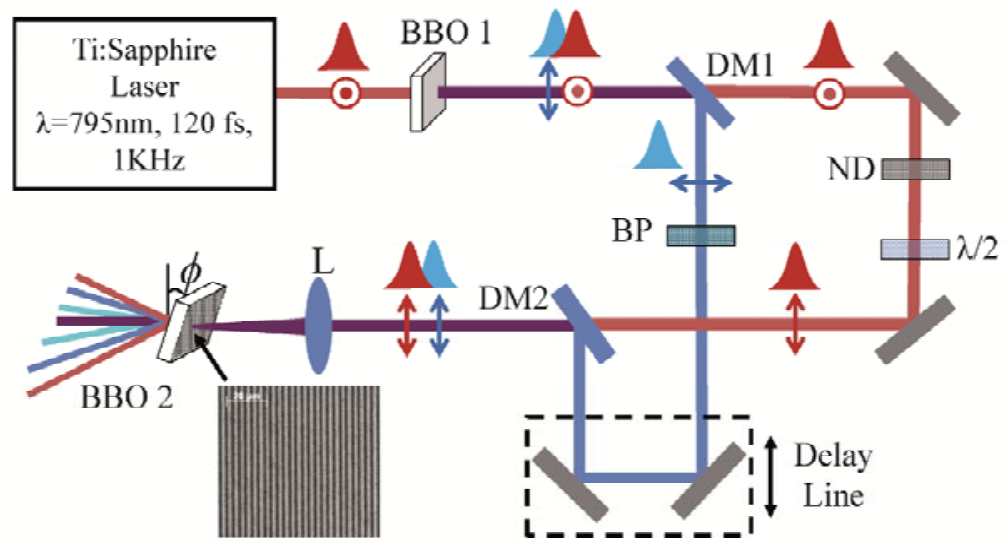

Figure 4.6. Experimental arrangement for THG in a micro-structured BBO crystal. DM: dichroic mirror, L: lens, ND: neutral density filter, BP: band-pass filter.

As can be seen in the diagram, the same elements as in the previous chapter are needed: a delay line and a half-wave plate to rotate the polarization of one of the beams. Again, we rotated the fundamental; there is no particular reason for this, it was simply because this was the optical element available in the laboratory.

Two dichroic mirrors, with $95 \%$ reflection in the blue and $98 \%$ transmission in the infrared, were used in order to separate the fundamental from the second harmonic after BBO 1 (DM1 in 
Figure 4.6), and to make the beams collinear (DM2) before entering the second nonlinear crystal (BBO 2).

The input energy was reduced to $70 \mu \mathrm{J}$, with a half-wave plate and a linear polarizer, before entering BBO 1 . The measured conversion efficiency in this crystal was $2 \%$. This result is very low but is not surprising because the input intensity was also low $\left(1.2 \mathrm{GW} / \mathrm{cm}^{2}\right)$ and very distant from the intensity required for efficiency saturation (see Figure 3.10 a)). The central wavelength $(397.5$ $\mathrm{nm})$ and the spectral width of the second harmonic pulses $(\sim 2.4 \mathrm{~nm})$ were measured with the same spectrometer used in the previous chapter (HR4000, Ocean Optics).

The nonlinear crystal BBO 2 was placed on a positioning mount that allowed us to change rotation and tilt. The influence of the tilt angle in phase matching will be explained later, but the use of the rotation is mandatory to adjust the phase-matching angle at normal incidence. As we explained in the previous chapter, a fine adjustment with the spectrometer was necessary to adjust the maximum phase-matching efficiency at the central wavelength of the laser. In Figure 4.6 the polarization of the beams is also shown. In the Type I interaction the two beams, fundamental and second harmonic, are ordinary waves; in this case they were p-polarized waves, with their polarization perpendicular to the grating grooves, as mentioned before.

Since the grating dimensions were much smaller than the total surface of the crystal, and therefore smaller than the diameter of the laser beam $\left(9 \mathrm{~mm}\right.$ at $1 / \mathrm{e}^{2}$ criterion), a lens was used to reduce the beam size. We used an achromatic doublet with a focal length of $40 \mathrm{~cm}$; $\mathrm{L}$ in Figure 4.6. With a CCD camera we measured the size of both beams at the entrance of the crystal to be sure that both fitted inside the grating. We avoided the focus of the lens just to make sure that we were not damaging the crystal. At the grating plane, the diameter size of the biggest beam (the second harmonic) was $500 \mu \mathrm{m}$.

\subsubsection{First Experimental Observations}

As the two co-propagating beams enter the micro-structured nonlinear crystal, the diffraction grating produces the off-axis propagation of multiple beams (diffraction orders). We have represented this experimental situation in Figure 4.6. The angles of each of these beams with the incidence direction, at the exit of the crystal, were measured for both the fundamental and the second harmonic by intercepting them on a screen.

\begin{tabular}{|c|c|c|c|c|}
\hline \multirow[b]{2}{*}{ Angle } & \multicolumn{2}{|l|}{ Fundamental } & \multicolumn{2}{|c|}{ Second harmonic } \\
\hline & Order $1 \tilde{\alpha}_{1}^{\omega_{1}}$ & Order $2 \tilde{\alpha}_{2}^{\omega_{1}}$ & Order $1 \tilde{\alpha}_{1}^{\omega_{2}}$ & Order $2 \tilde{\alpha}_{2}^{\omega_{2}}$ \\
\hline Measured & $14.9^{\circ}$ & $34.1^{\circ}$ & $7.4^{\circ}$ & $14.9^{\circ}$ \\
\hline Calculated & $13.1^{\circ}$ & $27.0^{\circ}$ & $6.5^{\circ}$ & $13.1^{\circ}$ \\
\hline
\end{tabular}

The results are summarized in Table 4.1, where we have also included the theoretical values calculated following the well known relation:

$$
\sin \tilde{\alpha}=m \frac{\lambda}{d}
$$


where $\tilde{\alpha}$ is the external angle of diffraction, $m$ is the diffraction order, and $d$ is the grating spacing. Note that we shall have a different angle for each frequency and diffraction order. However, owing to the wavelength relation, the first order of diffraction for the fundamental $(\omega)$ will share the same $\tilde{\alpha}$ with the second order for the second harmonic $(2 \omega)$.

Up to now we had considered only external angles, but for phase-matching calculations we needed to use the internal diffraction angles. Therefore, at this point it is also good to replace the wavelength by the frequency in the equations since this will make the theoretical treatment easier to understand. Thus, applying the wavelength-frequency relation and also introducing the frequencydependent refractive index, we obtained the following relation for the internal angles of diffraction:

$$
\sin \alpha_{m}^{\omega_{i}}=m \frac{2 \pi c}{\omega_{i} n_{o}^{\omega_{i}} d}
$$

The tilt of the crystal, $\phi$, was changed finely while we observed the beams projected on the screen. The value $\phi_{0}=0$ corresponds to the normal incidence position. In the zero-order of diffraction, where the second harmonic and the fundamental travel together, collinear SFG occurs, providing that the temporal overlap is achieved. Recall that the cut-angle of the crystal was such to phase-match this process: $\mathrm{O}_{795}+\mathrm{O}_{397.5}=e_{265}$ at normal incidence. The most convenient way of detecting this signal would be to have a band-pass filter centred in the $266 \mathrm{~nm}$ wavelength range, which removes the second harmonic and the fundamental. However, at the time that filter was not available in the laboratory so we used a UV-grade fused silica prism to separate the beams. With the spectrometer we recorded a spectrum of the UV radiation, displayed in Figure 4.8.

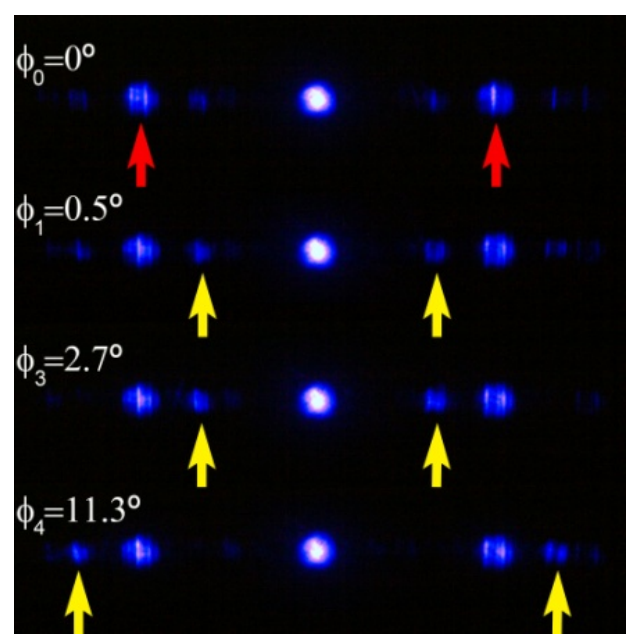

Figure 4.7. Emerging beams projected on a screen for different tilt angles $\phi_{i}$. Red arrows point to the second harmonic and yellow arrows point to the noncollinearly generated third harmonic, with its intensity maximized for the given tilt angle. The bright spot in the middle of each frame is the zero order of diffraction for both beams.

The use of the prism has some disadvantages, as argued in the previous chapter. However, these do not affect the spectrum of the pulses. The beams can be seen in Figure 4.7. The red arrows point to the first diffraction order for the second harmonic. Note that other weak beams appear between the orders 0 and \pm 1 : these are noncollinearly generated third-harmonic beams. They form external angles with the 0 order of $\pm 4^{\circ}$. 
The next step was to finely change the tilt angle from the normal incidence position, in the direction indicated in Figure 4.9. Close to this position, at $\phi_{1}=0.5^{\circ}$, the intensity of these other thirdharmonic beams was maximized. While we continued increasing the tilt, these beams vanished but another two appeared, propagating at external angles of $\pm 9.2^{\circ}$. The maximum of these signals was obtained for a tilt angle of $\phi_{2}=2^{\circ}$. We increased the tilt again up to $\phi_{3}=2.7^{\circ}$ and another two beams emerging at the position $\pm 4^{\circ}$ were optimized. At first glance they appear to be more intense than the previous ones. Again, we carefully adjusted the delay position, and the energy per pulse of the beams increased up to a maximum of $35 \mathrm{~nJ}$ in each of them. A spectrum recorded at one of these positions is shown in Figure 4.8.

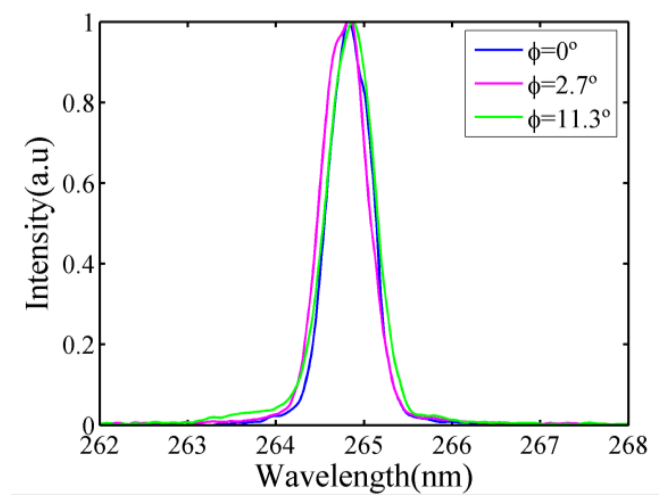

Figure 4.8. Third harmonic spectra recorded at the different tilt angles indicated in the legend.

A further increase in the crystal tilt $\left(\phi_{4}=11.3^{\circ}\right)$ led to the generation of another two third harmonic beams propagating at angles of $\pm 9.2^{\circ}$. These two beams were very sensitive as regards the delay position, and it was necessary to finely adjust it in order to obtain the maximum pulse energy: $10 \mathrm{~nJ}$. The spectrum of one of these beams was also analysed, and is also shown in Figure 4.8. In this case, some spatial chirp appeared, which could be attributed to the angular dispersion induced by the grating in the noncollinear beams.

As can be seen in Figure 4.7 the spatial profile of the diffracted beams is not very good, revealing some structure. This is due to possible errors in the movement of the motors during the grating recording, because they do not have a closed-loop (i.e. a feedback of their position after each movement). As a consequence of these positioning mistakes, the grating spacing is not perfectly uniform. In gratings with a greater groove spacing, this effect is reduced, but in our case the writing parameters, defined by the nonlinear interaction, were quite demanding for this kind of motor.

\subsubsection{Theoretical Description}

In this section we offer a detailed description of the beams inside the micro-structured crystal in order to clarify the noncollinear process that leads to the generation of each of the third harmonic signals observed. We shall start by introducing the phase-matching condition for the process of SFG:

$$
\mathbf{k}_{m}^{\omega_{1}}+\mathbf{k}_{n}^{\omega_{2}}=\mathbf{k}_{(m, n)}^{\omega_{3}}
$$


where $\mathbf{k}_{m}^{\omega_{1}}$ and $\mathbf{k}_{n}^{\omega_{2}}$ are the wave vectors for the fundamental and the second harmonic at the corresponding diffraction orders $m$ and $n$ respectively (inside the crystal), and $\mathbf{k}_{(m, n)}^{\omega_{3}}$ is the wave vector for the third harmonic generated. We can write these equations in terms of the indices of refraction, taking into account the energy conservation law: $\omega_{3}=\omega_{1}+\omega_{2}$, where the second harmonic frequency is twice the frequency of the fundamental, $\omega_{2}=2 \omega_{1}$, and where: $\omega_{3}=3 \omega_{1}$. We have that:

$$
\mathbf{k}_{m}^{\omega_{1}}=\frac{\omega}{c} n_{o}^{\omega_{1}} \mathbf{u}_{1} \mathbf{k}_{n}^{\omega_{2}}=\frac{2 \omega}{c} n_{o}^{\omega_{2}} \mathbf{u}_{2} \quad \mathbf{k}_{(m, n)}^{\omega_{3}}=\frac{3 \omega}{c} n_{e}^{\omega_{3}}(\theta(\phi, \chi)) \mathbf{u}_{3}
$$

In the case of perfect phase matching, and for Type I SFG, the vector equation in (4.3) can be expressed in terms of the following scalar equations, projecting $\mathbf{k}_{m}^{\omega_{1}}$ and $\mathbf{k}_{n}^{\omega_{2}}$ over $\mathbf{k}_{(m, n)}^{\omega_{3}}$ :

$$
\left\{\begin{array}{l}
3 n_{e}^{\omega_{3}}(\theta(\phi, \chi))=n_{o}^{\omega_{1}} \cos \left(\alpha_{m}^{\omega_{1}}-\chi\right)+2 n_{o}^{\omega_{2}} \cos \left(\chi-\alpha_{n}^{\omega_{2}}\right) \\
n_{o}^{\omega_{1}} \sin \left(\alpha_{m}^{\omega_{1}}-\chi\right)+2 n_{o}^{\omega_{2}} \sin \left(\alpha_{n}^{\omega_{2}}-\chi\right)=0
\end{array}\right.
$$

Figure 4.9 represents the exact definition of the angles inside the crystal. As the two beams enter the crystal, they will be diffracted by the grating along the directions defined by Eq. (4.2). However, if the crystal is tilted with respect to the normal position of incidence, the beams will also undergo refraction, and the diffraction plane (the plane where the diffracted beams for a given wavelength are contained) will be tilted by some angle, $\beta(\phi)$, with respect to the initial plane, $\beta(\phi)=\arcsin \left(\sin (\phi) / n_{0}^{\omega_{i}}\right)$. Note that the c-axis of the crystal is in the $\mathrm{y}-\mathrm{z}$ plane, and that the tilt rotations are made with respect to the $\mathrm{x}$-axis.

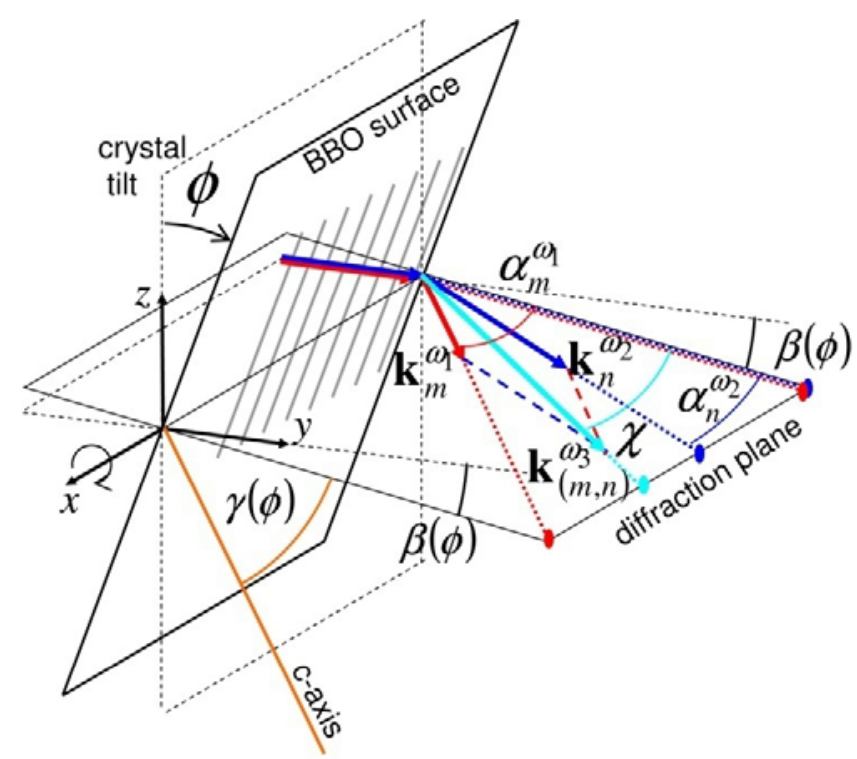

Figure 4.9. Diagram representing the wave-vectors and the angles definition inside the crystal. For the sake of clarity, only half of the diffraction plane is shown; positive diffraction orders. 
For simplicity, we shall assume that the diffraction plane for the two incident wavelengths will be the same. This means that we are neglecting the dependence of the index of refraction on the wavelength at this point, considering $n_{0}^{\omega_{1}} \cong n_{0}^{\omega_{2}} \cong 1.7$.

Evidently, the angle forming the c-axis with the diffraction plane will change accordingly: $\gamma(\phi)=\gamma_{0}+\beta(\phi)$, where $\gamma_{0}$ corresponds to the case $\phi=0$ and equals the crystal's cut-angle, which was $44.7^{\circ}$. Finally, the angle $\theta(\phi, \chi)$, which is the angle formed by the third harmonic beam and the c-axis, not represented in the Figure 4.9, depends on both $\gamma(\phi)$ and $\chi$, through the equation

$$
\cos \theta(\phi, \chi)=\cos \gamma(\phi) \cos \chi
$$

It is important to recall that by changing the tilt angle, $\phi$, we change the phase-matching conditions (see Eq. (4.5)), benefiting one diffraction plane with respect to the other.

The most intense noncollinear third-harmonic beams will be generated when in the SFG process the zero-order of diffraction for the fundamental or for the second harmonic is involved simply because of the larger fraction of energy that travels along this direction, owing to the efficiency of the grating (see Figure 4.5).

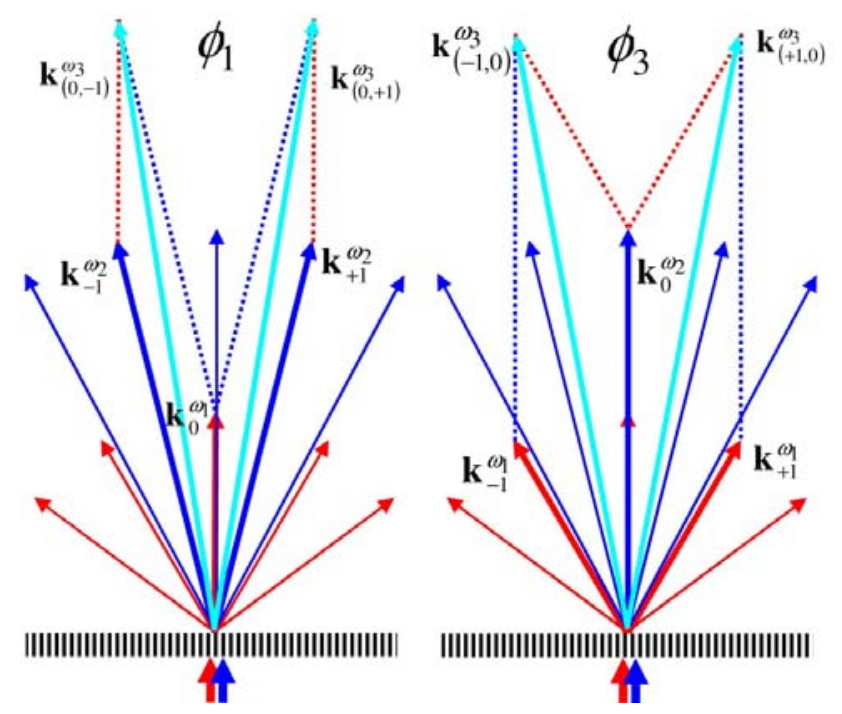

Figure 4.10. Sketch of the beams inside the crystal, indicating two different noncollinear processes involving the zero order of diffraction of the fundamental/second harmonic and the first order of the second harmonic/fundamental, respectively.

We used the scalar phase-matching conditions to calculate the tilt angles of the crystal, for which phase matching can occur between the zero order of the fundamental/second harmonic and the first order of the second harmonic/fundamental, respectively. We also calculated the external angle at which the beams emerged from the crystal. Both values can be compared with the angles at which we observed the generation of intense third harmonic beams experimentally, as described above; this helps to identify the mechanisms leading to the third harmonic beams observed in the previous section. 
In Table 4.2 we see that the processes involving the zero order for one wavelength and the order $m$ for the other generate the sum of both at approximately the same emerging angle, but for a different crystal tilt.

\begin{tabular}{ccccc} 
& $\tilde{\chi}_{(m, n)}$ th. & $\tilde{\chi}_{(m, n)}$ exp. & $\phi_{i}$ th. & $\phi_{i}$ exp. \\
\hline $\mathrm{k}_{0}^{\omega_{1}}+\mathrm{k}_{1}^{\omega_{2}}$ & $4.3^{\mathrm{o}}$ & $4.4^{\mathrm{o}}$ & $0.5^{\mathrm{o}}$ & $0.5^{\mathrm{o}}$ \\
$\mathrm{k}_{0}^{\omega_{1}}+\mathrm{k}_{2}^{\omega_{2}}$ & & & & \\
$\mathrm{k}_{0}^{\omega_{2}}+\mathrm{k}_{1}^{\omega_{1}}$ & $4.7^{\circ}$ & $9.2^{\mathrm{o}}$ & $1.9^{\mathrm{o}}$ & $2^{\mathrm{o}}$ \\
$\mathrm{k}_{0}^{\omega_{2}}+\mathrm{k}_{2}^{\omega_{1}}$ & & $4.3^{\mathrm{o}}$ & $2.3^{\mathrm{o}}$ & $2.7^{\mathrm{o}}$ \\
& $8.8^{\mathrm{o}}$ & $9.2^{\mathrm{o}}$ & $9.4^{\mathrm{o}}$ & $11.3^{\mathrm{o}}$
\end{tabular}

Table 4.2. Calculated (th.) and experimentally measured (exp.) crystal tilts ( $\left.\phi_{i}\right)$ and emergence angles of the third harmonic pulses $\left(\tilde{\chi}_{(m, n)}\right)$.

Figure 4.11 shows the angles between the fundamental and the second-harmonic beams with which phase matching is achieved as a function of crystal tilt, calculated by applying the phasematching conditions, Eq. (4.5). The blue curve corresponds to the processes involving the zero order for the fundamental, $\alpha_{0}^{\omega_{1}}=0$, and the $n$ order for the second harmonic, $\alpha_{n}^{\omega_{2}}$, while the red curve is the opposite. Filled circles are the experimental measurements and match the calculated ones fairly well.

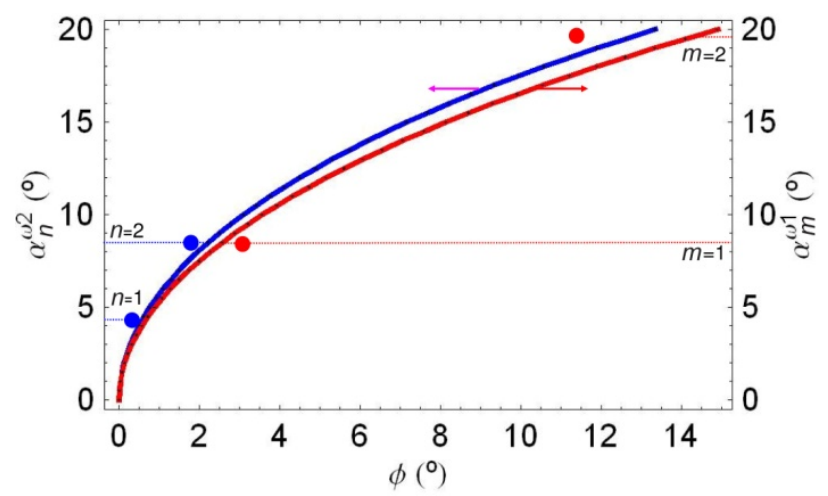

Figure 4.11. Noncollinear phase-matching curves. See the text for further details.

\subsection{Application of the Micro-Structured BBO to Intensity Cross- Correlation Measurements}

As explained in the previous chapter, in addition to the phase-matching conditions in the SHG and SFG processes the GVM between the interacting pulses must also be taken into account. When the group velocity is matched it can be ensured that the pulses will travel together during their propagation inside the nonlinear crystal. However, it is not possible to achieve this in a collinear configuration, as we have also explained. 
Nonetheless, there are experimental approaches to compensate for the GVM inside the crystal, based on noncollinear phase matching; in particular for SHG see references [57, 58]. Furthermore, the noncollinear phase-matching technique allows an increase to be made in the amplification bandwidth in optical parametric amplifiers. Moreover, the efficiency of parametric processes can be improved by combining noncollinear phase matching with pulse-front tilting [59]. However, in comparison with the approaches used in the latter references our device has the advantage of being compact, since the element that allows the nonlinear propagation is integrated in the nonlinear crystal itself, and hence no complicated beam alignment procedure is required.

Returning to the disturbing effects in the SFG pulses, the GVM, apart from reducing the efficiency of the process, has its own implications when the SFG process is used as a means to characterize the ultrashort pulses through cross-correlation measurements. The time-smearing effect, already introduced in Chapter 3 (Sect. 3.6.2), artificially elongates the cross-correlation trace. Therefore, if we wish to avoid any distortion in the trace the crystal used must have a maximum thickness determined by the condition (3.23). However the micro-structured crystal offers the possibility of avoiding, or at least alleviating, the disturbing effect of the GVM.

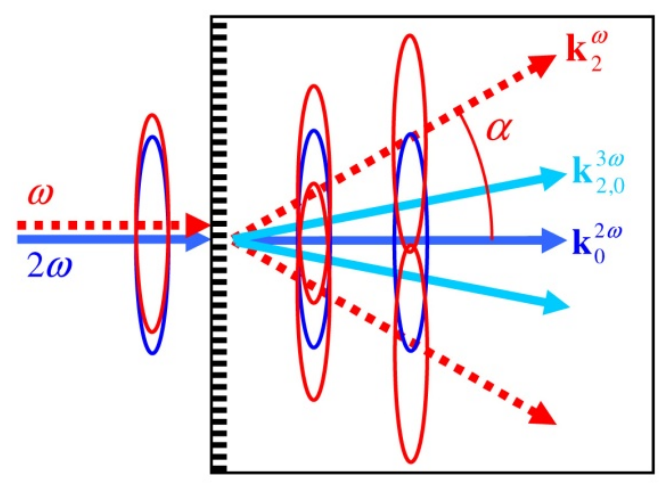

Figure 4.12. Diagram representing the noncollinear SFG process induced by the grating, showing pulse tilting and effective group-velocity matching.

After propagating through an angularly dispersing element, such as a diffraction grating, ultrashort laser pulses undergo a pulse-front tilt that depends on the grating parameters, the conditions of incidence, and the distance to the grating [60]. For normal incidence on the grating and just after this element, the tilt angle approximately equals the diffraction angle. This means that inside the micro-structured crystal the pulse-fronts of all the diffraction orders will be nearly parallel to the pulse-fronts of the incident pulses, see Figure 4.12.

Owing to this particular way of propagation, the effective group velocity of the pulses travelling in noncollinear directions becomes reduced by a factor $\cos \alpha$ when they combine with other pulses travelling along the 0 order (undiffracted), $\alpha$ being the angle between the interacting pulses. Thus, if the pulse that has the largest group velocity (the infrared, $v_{g}=0.178 \mu \mathrm{m} / \mathrm{fs}$ ) travels in an out-ofaxis direction, its effective group velocity can be equal to that of the second harmonic travelling along the axis $\left(v_{g}=0.168 \mu \mathrm{m} / \mathrm{fs}\right)$. The $\mathrm{SFG}$ process will be effectively matched for $\alpha=19.52^{\circ}$. In the case of the grating recorded in the $\mathrm{BBO}$, this condition is fulfilled between the zero order of the second harmonic and the second order of the fundamental:

$$
v_{g}^{\omega_{2}}=v_{g}^{\omega_{1}} \cos \alpha_{m}^{\omega_{1}}
$$


In fact, the second order of diffraction for the fundamental propagates inside the nonlinear crystal at an angle slightly lower than that desired to fulfil condition (4.7) (the measured external angle was $\tilde{\alpha}_{2}^{\omega_{1}}=34.1^{\circ}$ (see Table 4.1), which corresponds to an internal angle of $19.25^{\circ}$ ). In sum, for the $\mathbf{k}_{2}^{\omega_{1}}+\mathbf{k}_{0}^{\omega_{2}}=\mathbf{k}_{(2,0)}^{\omega_{3}}$ process the GVM is expected to be reduced.

We were therefore prompted to apply the micro-structured crystal to intensity cross-correlation measurements and thereby obtain a temporal characterization of the second harmonic pulses. It is important to note that no other element needs to be introduced except for a detection system for the third harmonic signal. On this occasion, we used a GaP photodiode to measure the power of the third harmonic signal instead of the spectrometer. The photodiode was placed in such a way that only the emergent sum frequency generated at the external angle $\tilde{\chi}=9.2^{\circ}$ would be detected.

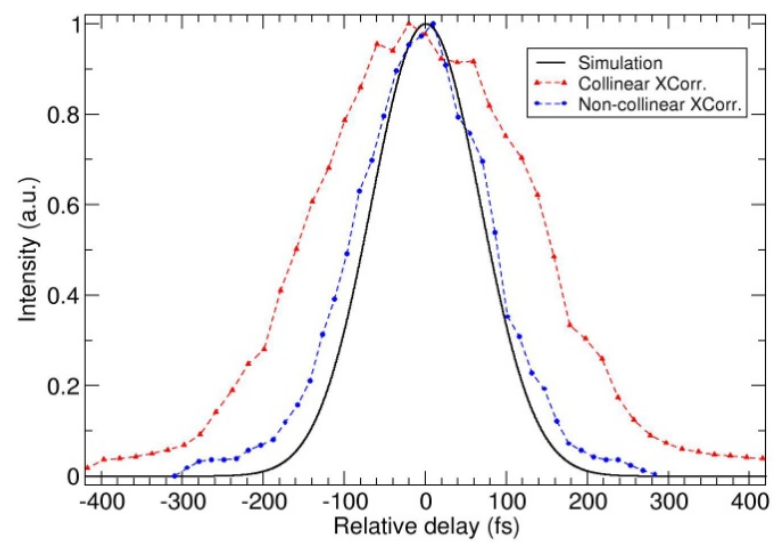

Figure 4.13. Cross-correlation traces obtained with the micro-structured nonlinear crystal for collinear — red line- and noncollinear - blue line-SFG processes. The solid black line represents a theoretical cross-correlation trace computed assuming GVM=0.

We proceeded in a similar way as in the previous chapter, recording the power in the third harmonic signal for different delay positions. The results obtained are summarized in Figure 4.13, where we show two cross-correlation traces for the second harmonic generated in the $0.5 \mathrm{~mm}$ BBO performed in the mircro-structured BBO: 1-mm thick. The red line corresponds to the trace obtained in a standard collinear configuration, (the tilt angle was $\phi=0^{\circ}$ : normal incidence). The GVM is not compensated and the trace is broad due to the time-smearing effect. The trace in blue was obtained in the effective group-velocity compensation configuration described above (corresponding to a tilt angle of $\phi=11.3^{\circ}$ ). A theoretical curve was included for comparison; in this case the GVM is equal to 0 . To generate this curve, we used the one-dimensional numerical model described in the previous chapter [37] to compute the second harmonic and the fundamental pulses emerging from the first crystal (BBO 1). Then, a cross-correlation trace was simulated, assuming $\mathrm{GVM}=0$. The agreement of this trace with the one generated in the noncollinear configuration was fairly good thus demonstrating an effective GVM compensation.

\subsection{Conclusions}

In this part of the work, we combined the use of femtosecond pulses both for high-precision microfabrication in crystals and for their application in nonlinear optics. 
First, we used ultrafast micro-structuring techniques to integrate a $1 \mathrm{D}$ relief grating on the surface of a BBO nonlinear crystal. The excellent control of the laser parameters, energy and scanning velocity made it possible to minimize the groove size, which afforded us the possibility of reducing the grating spacing to $3.5 \mu \mathrm{m}$. Following its characterization (with a light and an AFM microscope), the grating was used to generate the sum frequency between the second harmonic and the fundamental Ti:Sapphire laser femtosecond pulses noncollinearly. Correct choice of the grating parameter, $d$, allowed the propagation angle between the fundamental and the second harmonic to be such that the GVM could be effectively matched for certain noncollinear configurations. Moreover, the application to intensity cross-correlation is also demonstrated.

Apart from the above-mentioned application of this device, although efficiency is low, it also has some applicability as a compact beam splitter or as a signal multiplexer, generating multiple waves at the fundamental, second and third harmonic frequencies.

Furthermore, the proposed technique offers some adaptability because the GVM of different noncollinear phenomena may be adjusted by changing the writing parameters. In particular, this idea could be applied to generate optical parametric amplification noncollinearly in the visible part of the spectrum. In this process, as a consequence of the tilting of the beams thanks to the grating the interaction length could be increased, thus benefiting the transfer of energy from the pump to the signal wave.

The main drawback is the low efficiency of the grating due to scattering losses in the ablation grooves. However, we anticipate that such losses could be reduced to a considerable extent by recording the grating at some depth inside the crystal by optical breakdown. In this way, the area affected can be smaller while the index of refraction is significantly modified.

To our knowledge, the integration of diffractive elements in periodically poled materials (crystals with a periodic inversion of the ferroelectric domains $[33,61]$ ) has not yet been explored. Even the micro-structuring of random quadratic media could be of great interest provided that phase-matching conditions in this case are less restrictive than making use of birefringence [62, 63]. Noncollinear interaction between femtosecond pulses in random quadratic media has been applied to the temporal characterization of ultra-short pulses [64]. As example, the integration of a grating in one of these crystals could be of interest to self-induce the noncollinear interaction inside the crystal. 



\section{Chapter 5}

\section{Second- and Third-Harmonic Generation with Diffractive Lenses}

\subsection{Introduction}

This chapter focuses on the generation of the second and third harmonics of femtosecond laser pulses. These phenomena were introduced theoretically in Chapter 2, and generated experimentally in Chapters 3 and 4. In Chapter 3 we analyzed the second-harmonic signal for its optimization according to the characteristics of our laser system, while in Chapter 4, a home-made diffractive element helped us, on the one hand, to tailor the harmonics generated and, on the other, to find an application in intensity cross-correlation measurements. In this chapter we shall also combine a diffractive element with these two nonlinear processes: in this case, our aim was to generate radiation in the same blue and UV regions but with new features. The main idea is to demonstrate that a diffractive lens (an optical element that will be introduced in the next section) can be a useful tool for obtaining tunability in the second- and third-harmonic signals generated in nonlinear crystals. As mentioned in previous chapters, the possibility of extending the available sources to new regions in the electromagnetic spectrum is always of great interest within the laser community [65-67]. 
The experiments reported in the present chapter, as well as those described in the next chapter, were developed in collaboration with the Group de Recerca d'Optica at the Universitat Jaume I in Castelló, although all the experimental set-ups were implemented at our laboratory.

As far as we are aware, very little work has been carried out in relation to the application of diffractive optics to these nonlinear processes. In experiments with nanosecond pulses a Fresnel Zone Plate (as we shall explain later, this optical element can be considered a poor diffraction efficiency diffractive lens) was applied to enhance the efficiency of SHG up to a factor of two and, more surprisingly, up to a factor of 16 in the third harmonic [68]. Moreover, at the time when we were performing our experiments other researchers demonstrated an application in femtosecond second-harmonic microscopy [69].

The chapter is organized as follows: first we shall briefly introduce the diffractive lens. Then, we shall describe the different experiments carried out at the laboratory and discuss the results obtained. To start, we initially demonstrate the tunability in the second-harmonic signal generated in nonlinear crystals. Then we study the dependence of the phenomenon on crystal thicknesses, and after this we characterize the signal thus generated. To conclude, we confirm the tunability in the third harmonic generated as the sum of the second harmonic and the fundamental. We finish with the conclusions of the chapter.

\subsection{Diffractive Lenses}

Essentially, a Diffractive Lens (DL) is a focusing element that focuses light based on diffraction. This DL has some similarities with a Fresnel Zone Plate (FZP) but instead of the most common versions of a FZP [70], with several foci, the DL that we used was optimized to concentrate all the energy with high efficiency (theoretically 100\%) in only one focus when using monochromatic illumination. Other designs, for example, allow one to obtain a bifocal lens, also with high efficiency [71]. Let us look at the characteristics of the one we used in detail.
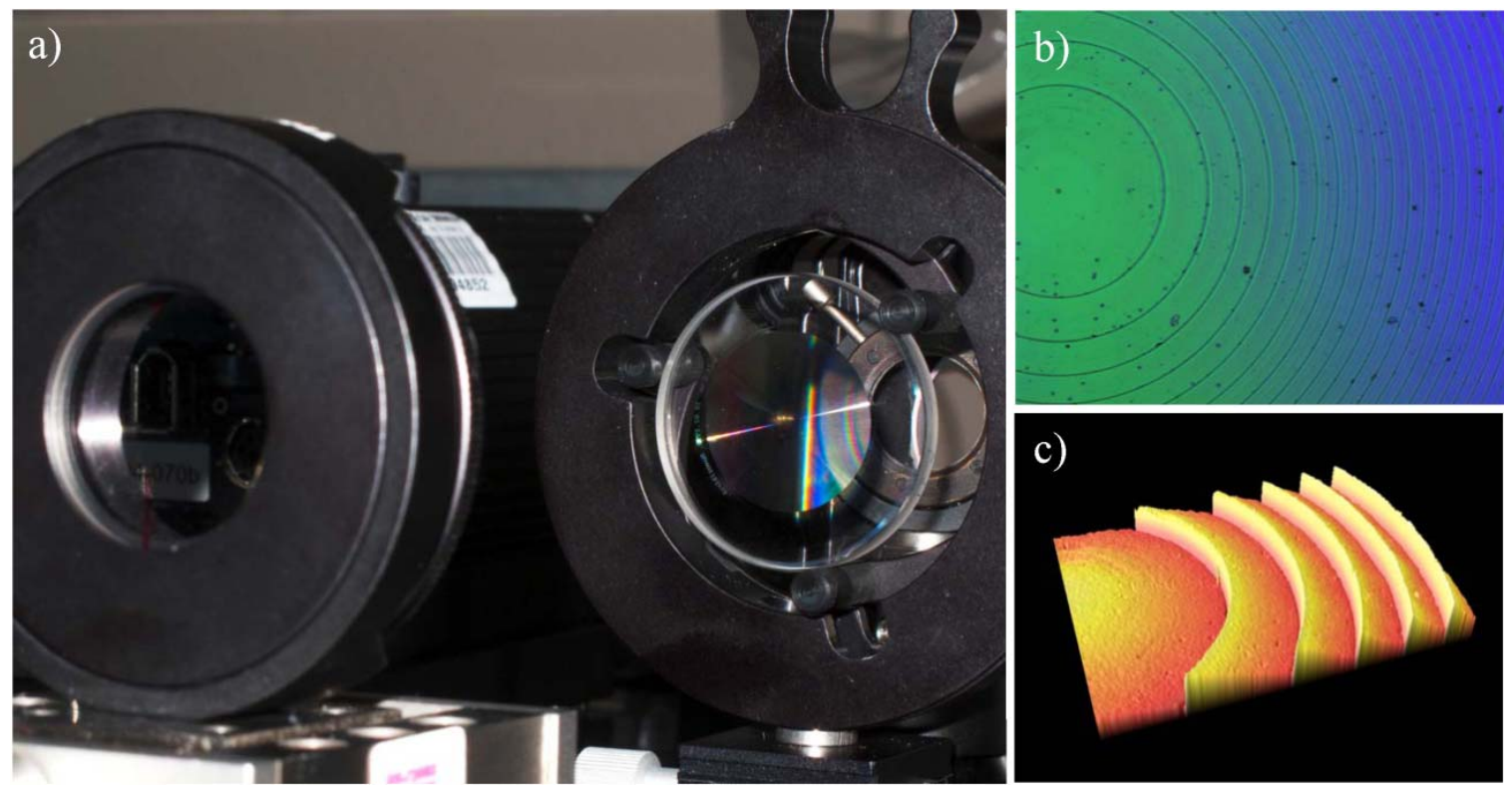

Figure 5.1. Different figure captions of the lens: a) Photograph of the lens mounted. b) Optical microscope image. c) Three-dimensional image of a detail of the lens from a SEM, taken from reference [72] with the permission of the authors. 
The simplest case of an FZP consists of a series of opaque and transparent rings with the same area. The interference among the transmitted parts of the beam leads to the focusing of the same, having a principal focal length of:

$$
f=\frac{R^{2}}{\lambda_{p}}
$$

$R$ being the radius of the inner ring and $\lambda_{p}$ the wavelength of the incident light. However, unlike a conventional refractive lens the FZP exhibits secondary focal lengths, following the relation $f_{m}=f / m$ (where $m$ is an odd integer), and with a maximum intensity decreasing with order $m$.

The kinoform, in contrast, is a continuous relief diffractive element, without opaque zones. In particular, the one we used was constructed with halftone mask technology (Institute of Automatics and Electrometry, Russia). As mentioned, the kinoform is a phase DL that provides the highest diffraction efficiency with continuous-wave illumination [71, 73]. Three different images of the lens can be seen in Figure 5.1. In panel a) we see a photograph where it is clear that there are no opaque and light zones. However, a rainbow-like pattern can be perceived, which suggests that some structure must be engraved on its surface. In b) an image taken with an optical microscope is shown, where a ring pattern appears. The radius $(R)$ of the rings is governed by expression (5.1). Moreover, some structure seems to be present inside the rings, but the microscope resolution is not high enough to resolve this pattern. Finally in panel c) a SEM picture shows a 3D image of the DL. This image clearly reveals one distinctive feature of the lens compared with the FZP: instead of light and dark zones, the lens is structured in zones with a $2 \pi$-radian phase shift.

Regarding the focusing properties of the lens, within the paraxial approximation, the generation of nonlinear effects by focusing a continuous-wave laser beam with a refractive lens or a DL with the same Numerical Aperture (NA) is expected to show a similar behaviour. However, when working with a non-monochromatic source, such as the ultrafast laser, strong differences appear due to the large spectral bandwidth of the pulsed radiation. In this case, the intensity distribution in the focal region of a DL is very different from that of a refractive lens. The chromatic aberrations of the kinoform are responsible for the difference, as we are about to see. In particular, for a refractive lens, chromatic aberrations are caused by the dispersion of the material [74] whereas for a DL they are due to the diffraction phenomena [75].

The focal length of the DL, $f$, is related to the wavelength by the expression:

$$
f_{\lambda}=f_{0} \frac{\lambda_{0}}{\lambda}
$$

where the subscript 0 denotes the reference wavelength. In our case the designed focal length was $f_{0}=150 \mathrm{~mm}$ for $\lambda_{0}=565 \mathrm{~nm}$. Therefore, for the central wavelength of the laser, $\lambda=795 \mathrm{~nm}$, the focal length would be $f_{\lambda}=106.6 \mathrm{~mm}$. Similarly, each wavelength of the incoming femtosecond laser pulse will be focused at a different position on the optical axis, generating as a consequence a continuum of foci. In fact, we can define a focal region for this lens as the difference between the focal length for the two wavelengths delimiting the laser spectral bandwidth $(\Delta \lambda)$. In the case of a refractive lens, the focal region is coincident with the Rayleigh length [25]. However, in this case, due to the above-mentioned strong chromatic aberration, we must define it as the region in the optical axis 
that contains the foci for all the wavelengths present in the laser bandwidth. The extension of this region can be calculated approximately as in [76]:

$$
\Delta f=f_{0} \frac{\Delta \lambda}{\lambda_{0}}
$$

for a DL manufactured in a material with low dispersion. For our $\sim 9 \mathrm{~nm}$ (at intensity FWHM) bandwidth pulses centred at $795 \mathrm{~nm}$, this results in $\Delta f \sim 1.3 \mathrm{~mm}$. This is several orders of magnitude larger than the focal region achieved with a singlet lens with refractive index, $n$, due to chromatic aberration [76]:

$$
\Delta f=-\frac{f_{0} \Delta \lambda\left(\frac{d n}{d \lambda}\right)_{\lambda_{0}}}{n-1}
$$

In the case of an achromatic doublet this axial extension is considerably reduced, because these types of lens are corrected to be free of chromatic aberrations.

To clarify this point in Figure 5.2 we plot the on-axis intensity distribution ${ }^{12}$ in the focal region as a function of wavelength for three different cases. Panel a) corresponds to the case of the refractive focusing of a laser pulses with a 9-nm intensity FWHM bandwidth. In panel b) we represent the case of focusing the same pulses but with a DL, with the same focal length for the central wavelength of the pulses. Finally, in panel c) we show the intensity distribution for a broadband laser ( $30 \mathrm{~nm}$ at intensity FWHM bandwidth) focused with the DL. In the maps we have also included the summation over wavelengths to clarify the effect of the extended focal region. In the representations it can be clearly seen how in the case of the DL the wavelengths are focused on different spatial points along the propagation axis, $z$. It is also clear that the extension of the focal region depends on the laser bandwidth.
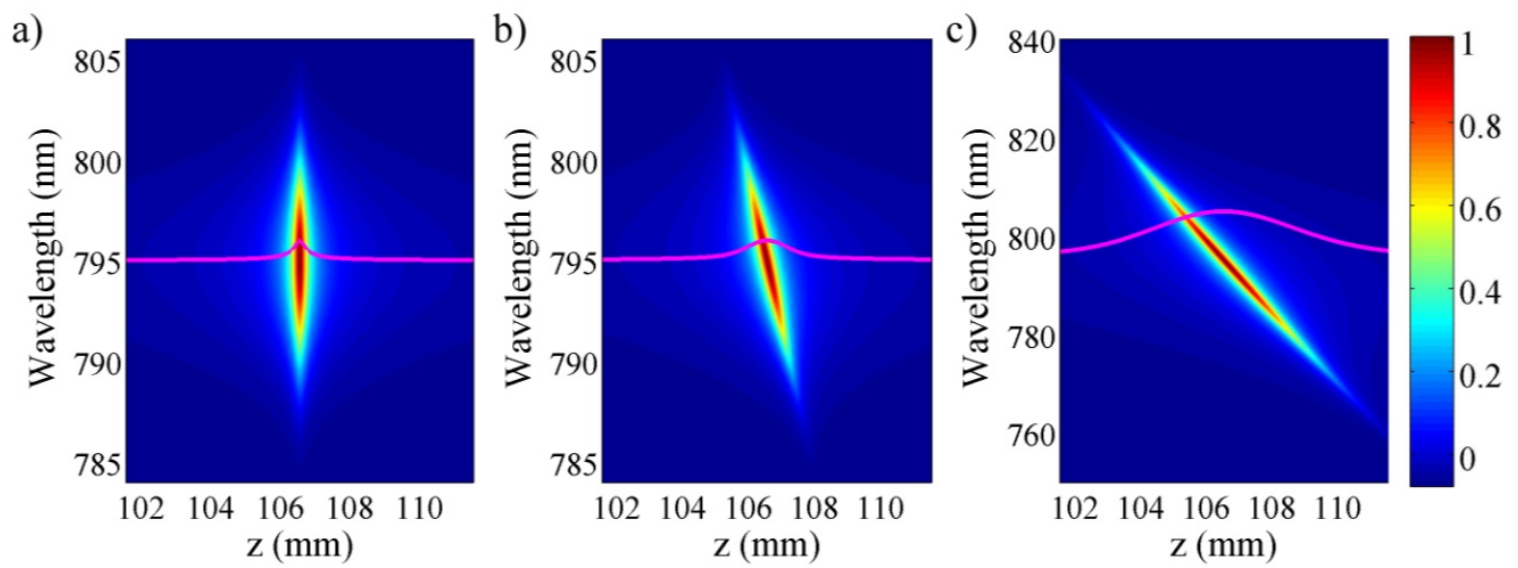

Figure 5.2. On-axis spectral intensity distribution. Panels a) and b) correspond to focusing 9-nm bandwidth pulses with a refractive lens and a DL respectively, with the same focal length. Panel c) corresponds to focusing a laser pulse of $30 \mathrm{~nm}$ bandwidth with the same DL. It should be noted that the wavelength scale has been increased in this latter case.

\footnotetext{
${ }^{12}$ We used the intensity definition given in Sect 5.3.2, Eq. (5.5), adapted for the case of the diffractive and the refractive focusing lens.
} 


\subsection{Wavelength Tunability in the Second-Harmonic Signal. Study of the Influence of Crystal Thickness}

The focal region of the lens is very important to see the effect of tunability, as well as other parameters of the nonlinear crystal that we shall describe briefly before passing on to describe the experiments. In particular, the extension of the focal region is important in the sense that it must be large in comparison with the nonlinear crystal thickness. In other words, the crystal must be thin enough to map all the wavelengths independently in order that only portions of the whole bandwidth will be focused inside the crystal at the same time. However, if the crystal is too thin the conversion efficiency will be very low, as we have already pointed out in previous chapters.

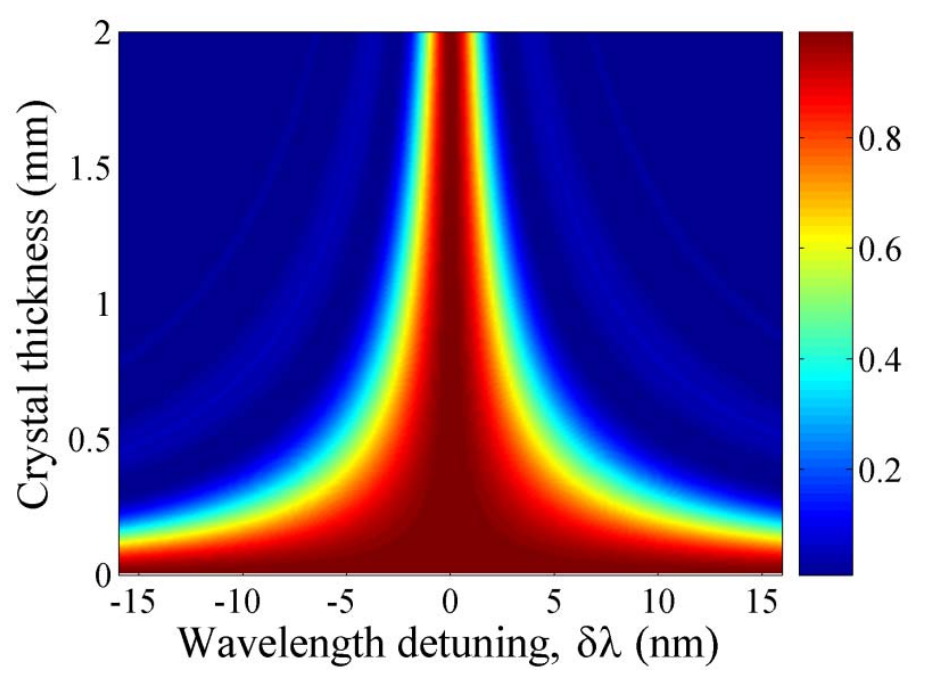

Figure 5.3. Colourmap representantion of the phase-mismatch factor as a function of the $B B O$ thickness and the laser bandwidth. The reference wavelength corresponds to the central wavelength of the laser $\lambda=795 \mathrm{~nm}$. The colourbar represents the efficiency in arbitrary linear units.

Another important issue to observe tunability is the bandwidth acceptance of the crystal, which has to cover the input laser spectra as much as possible so that the wavelengths of the fundamental can be efficiently doubled.

In this experiment, three different samples of BBO with thicknesses $40 \mu \mathrm{m}, 500 \mu \mathrm{m}$ and $2 \mathrm{~mm}$ were investigated. In Chapter 3 we depicted the phase-matching spectral acceptance at normal incidence for two of the three crystals, the $2-\mathrm{mm}$ and the $0.5-\mathrm{mm}$ thick ones. Here, in Figure 5.3 we have represented the phase-mismatch factor (Eq. (3.20)) as a function of the BBO crystal thickness and the laser bandwidth, as a detuning from the central wavelength. We can clearly see how the phase-matching bandwidth decreases with increasing crystal thickness. It can also be seen that only in the case of the 2-mm length crystal would a strong limitation in the up-converted spectrum be expected for the bandwidth of our laser ( $\sim 9 \mathrm{~nm}$ FWHM), and the spectral acceptance of the $500-\mu \mathrm{m}$ thick sample, approximately $10 \mathrm{~nm}$ at FWHM, fits the spectral width of the incident pulses. To conclude, note how in the case of the $40-\mu \mathrm{m}$ thick crystal the efficiency is nearly constant throughout the laser bandwidth. 
As can be extracted from Eq. (3.19) the phase-matching efficiency also depends on the angle detuning from the exact phase-matching one. Therefore, apart from dependence of the phasematching efficiency on wavelength, we also have to take into account the so-called angular acceptance of the crystal. The angular acceptance is defined as the angle interval that affords phasemismatching values higher than the intensity FWHM. When the fundamental beam is focused, a reduction in efficiency is produced as the focusing introduces different propagation directions (kvectors) from the original plane wave. This angular dependence of the phase-mismatching factor leads to the generation of asymmetric beams since one direction will be more affected by the angle detuning than the other. Photographs of the beams generated are shown in Sect. 5.4.3 and perhaps clarify this issue. In our case, the optical axis is contained in the horizontal plane and hence the limitation would appear in this direction. In Figure 5.4 we have plotted the phase-mismatch factor as a function of the NA instead of angle detuning. With the NA as reference, we can easily see the effect of focusing with the lenses that we used, which have NA $\sim 0.04$. If we look at the figure, even with the $0.5 \mathrm{~mm}$ crystal the efficiency decays significantly even with a lower NA.

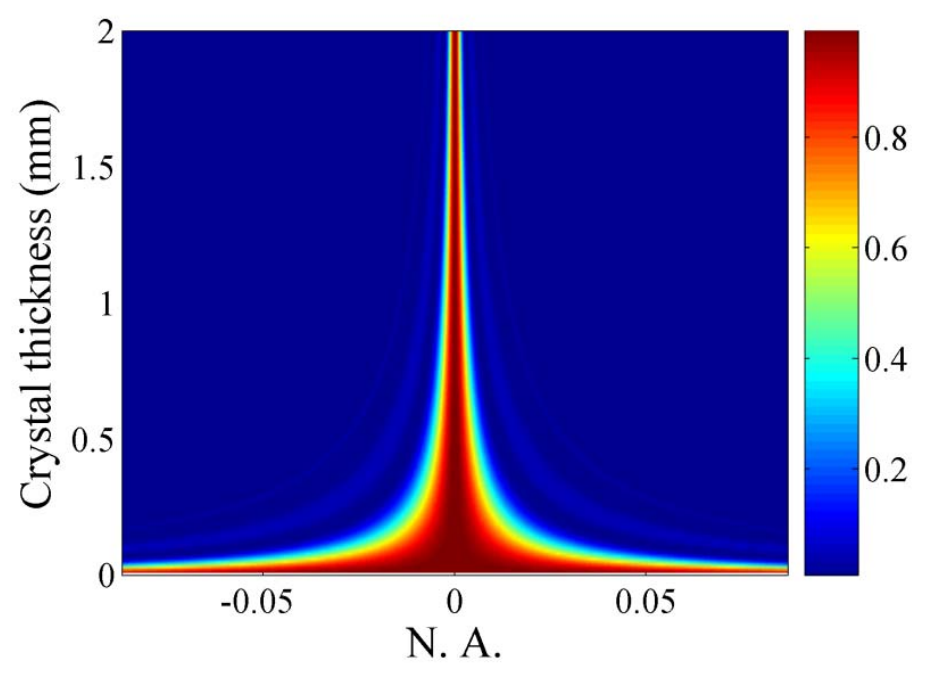

Figure 5.4. Colourmap representing the variation in the phase-mismatch factor with the NA of the incident beam in the nonlinear crystal as a function of crystal thickness. The colourbar represents the efficiency in arbitrary linear units.

\subsubsection{Experimental Set-Up and Analysis of the Results}

A diagram of the experimental set-up used for SHG with a DL is shown in Figure 5.5. After the energy-control system, we placed the DL coupled to a computer-controlled motorized linear stage that moved the lens along the propagation axis. The IR pulses were focused on BBO samples of different thicknesses in the way indicated in the figure. All the samples were cut for Type I SHG of the laser pulses at normal incidence $\left(\theta=29.4^{\circ}, \phi=90^{\circ}\right)$. Then, the beam was directed to two dichroic mirrors with $99.5 \%$ reflectance for $397 \mathrm{~nm}$. This is needed to separate the unconverted fundamental from the blue signal prior to being measured in the spectrometer and analysed. The DL was replaced by a conventional achromatic refractive lens with a focal length of $100 \mathrm{~mm}$ (Linos G063144525) when necessary for comparative purposes. The focal length of this lens was chosen in order to obtain the same NA as for the kinoform. The effect of the chromatic aberration of the DL is also shown in the diagram to help readers form an image in their minds of how this lens actually acts. 
We took a spectrum in each relative position of the crystal and the lens. The results are depicted in Figure 5.6, where the zero value corresponds to the position where the central wavelength of the laser is being focused in the middle of the nonlinear crystal, and the positive values correspond to the position when the lens is in the forward direction, so the lens-crystal distance is decreased.

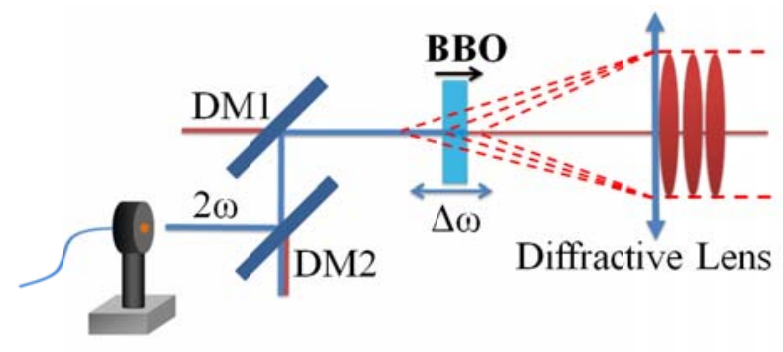

Figure 5.5. Depiction of the experimental set-up for SHG in nonlinear crystals with femtosecond pulses and a kinoform DL. DM1,2: Dichroic mirrors.

Each map in Figure 5.6 represents in the vertical direction the spectra of the second harmonic, and in the horizontal direction the relative position between the crystal and the lens. The figure is organized as follows: each row corresponds to a different crystal thickness, increasing from top to bottom: $40 \mu \mathrm{m}, 0.5 \mathrm{~mm}$ and $2 \mathrm{~mm}$. The first column corresponds to the maps recorded when the DL was replaced by the refractive lens, and they are presented here for comparative purposes. The column in the middle corresponds to the focusing with the DL, and the right column corresponds to the theoretical calculations, also for the DL. See Section 5.3.2 for further details in the calculations.

If we compare columns 1 and 2, we see how in the case of focusing with the DL the central wavelength changes when changing the relative distance, $z$, except in the case of the $2 \mathrm{~mm}$ crystal. This confirms that the central wavelength of the second harmonic signal can be tuned, when changing the lens-crystal distance, provided that the thickness of the crystal is adequate. In the case of the thick crystal, we can attribute the lack of tunability to the fact that its thickness is greater than the focal region of the lens $(\sim 1.3 \mathrm{~mm}$, defined in Sect. 5.2), and for any given position all the wavelengths are focused inside the crystal.

We also see how the measurements performed with the $40 \mu \mathrm{m}$ and $0.5 \mathrm{~mm}$ crystal, when focusing with the DL, are quite similar. In these two cases - maps b) and e) - the effect of tunability obtained when changing the lens-crystal distance can clearly be seen. Note that in both cases the crystal thickness is smaller than the focal region of the DL. Thus, for each position of the crystal the second-harmonic spectrum is determined by the wavelengths from the fundamental pulses that are being focused inside the nonlinear crystal. The range of tunability is $\sim 4 \mathrm{~nm}$, corresponding to the total available spectrum of the fundamental pulse ( $\sim 9 \mathrm{~nm}$ at intensity FWHM). We also would like to remark that no additional tilting or rotation of the crystal was performed when recording the maps. As the relative lens-crystal distance is increased, the second harmonic central wavelength decreases, as follows from Eq. (5.2).

As expected the effect of tunability does not appear when focusing with the refractive lens, panels a) d) and g). The spectral content of the second harmonic signal is the same in all the positions, and we assume the focus of this lens to be the same for all the wavelengths in the spectrum. 

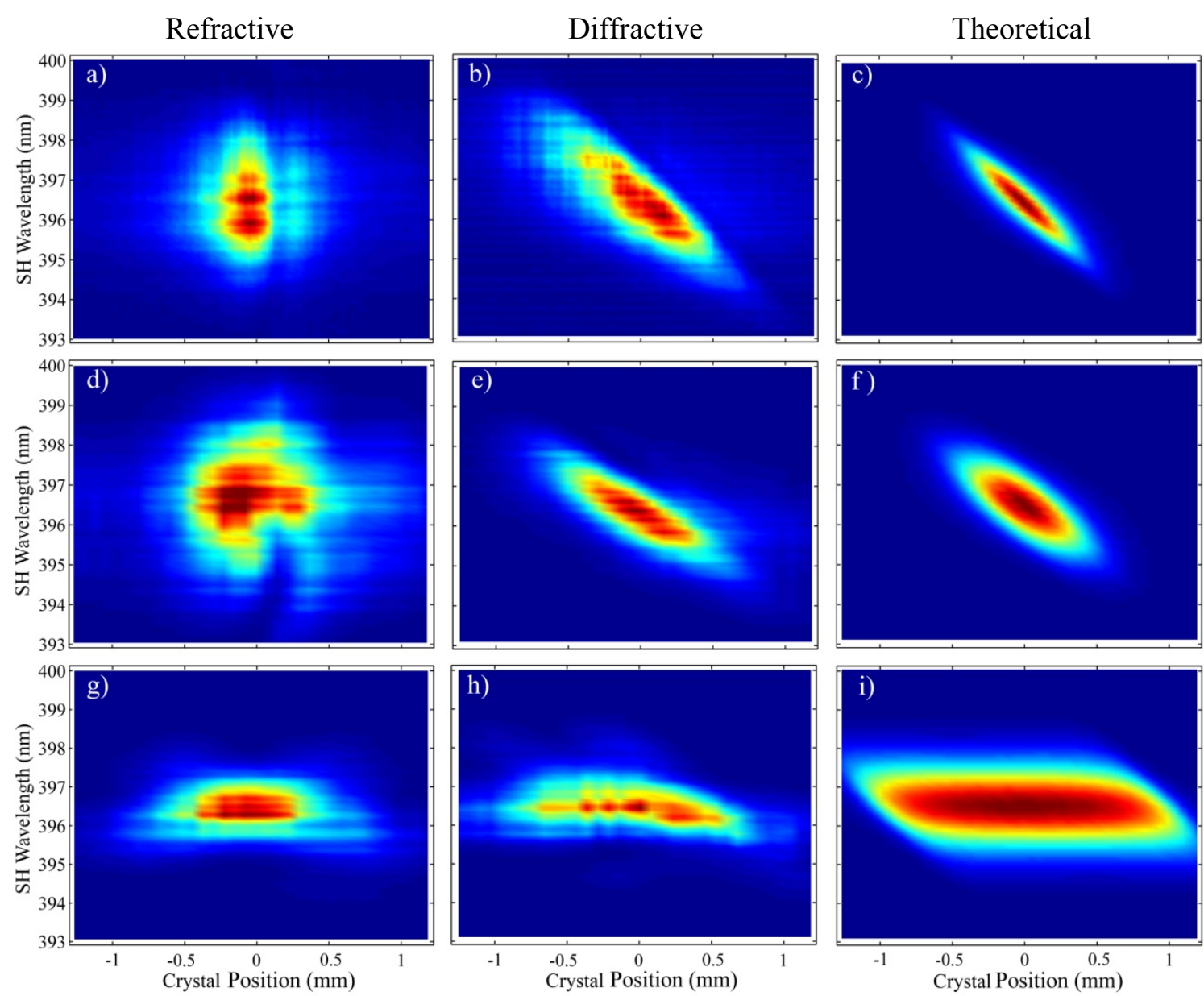

Figure 5.6. Experimental maps of SHG spectra taken when changing the lens-crystal distance in a), d) and g) focusing with a refractive lens and in b) e) and h) with the DL. The crystal thickness used were a) and b) a 40- $\mu \mathrm{m}$ thick crystal, d) and e) $0.5 \mathrm{~mm}$ and g) and h) $2 \mathrm{~mm}$. A theoretical calculation, when focusing with a DL, is shown in panels c), f) and i) for a $40-\mu m, 0.5-m m$ and 2-mm thickness crystal respectively.

If we look at maps a) and b), d) and e), another effect of the DL is remarkable; the combination of the strong chromatic effect of the lens and the limited size of the crystal make the system act as a band-pass optical filter for the wavelengths in the UV region. The second-harmonic bandwidth is clipped due to the chromatic aberration of the lens, and the effect of the distributed foci. In particular, for the $0.5-\mathrm{mm}$ thick BBO, the spectral width is $1.5 \mathrm{~nm}$ for the pulses generated with the $\mathrm{DL}$, and $2.4 \mathrm{~nm}$ in the case of the refractive focusing. Therefore, an enlargement of the pulse duration is expected due to this reduction in bandwidth. Assuming a transform-limited pulse, the temporal duration of second harmonic pulses, with a $1.5-\mathrm{nm}$ bandwidth, is $145 \mathrm{fs}$. For this case in particular, the temporal duration of the pulses was measured experimentally and these results are presented in Sect.5.4.1.

\subsubsection{Theoretical Model}

The wavelength dependence of the focal length for the kinoform lens can be written as Eq. (5.2), where $f_{0}$ is the focal length for the wavelength $\lambda_{0}$. In a rough approximation, the laser intensity in the focal region can be written, assuming a Gaussian shape for the incident beam [6], as: 


$$
I(r, z, \lambda)=\frac{2 P(\lambda)}{\pi w(z, \lambda)} \exp \left[-\frac{2 r^{2}}{w^{2}(z, \lambda)}\right]
$$

where $P(\lambda)$ is the spectral power for the wavelength $\lambda, r$ is the radial coordinate and $z$ is the coordinate along the lens axis (propagation axis), and $w(z, \lambda)$ is the beam radius, in which the dependence of the focal length on $\lambda$ is included as:

$$
w(z, \lambda)=w_{0}(\lambda)\left[1+\left(\frac{\lambda(z-f(\lambda))}{\pi w_{0}^{2}(\lambda)}\right)^{2}\right]^{1 / 2}
$$

Taking into account Eq. (5.2), the beam radius at the focus (beam waist), $w_{0}(\lambda)$, proves to be:

$$
w_{0}(\lambda)=\frac{\lambda f(\lambda)}{\pi w_{b}}=\frac{\lambda_{0} f_{0}}{\pi w_{b}}
$$

$w_{b}$ being the beam radius at the entrance of the DL.

The wave equations ${ }^{13}$ for the fundamental $\left(A_{F}\right)$ and the second harmonic $\left(A_{S H}\right)$ fields, assuming monochromatic plane waves propagating along the $\mathrm{z}$ axis (in the slowly varying amplitude approximation) [25], are:

$$
\begin{aligned}
& \frac{d A_{F}(z, \lambda)}{d z}=\frac{4 \pi i d_{e f f}}{\lambda n_{\lambda}} A_{S H}(z, \lambda) A_{F}^{*}(z, \lambda) e^{-i \Delta k z} \\
& \frac{d A_{S H}(z, \lambda)}{d z}=\frac{4 \pi i d_{e f f}}{\lambda n_{\lambda / 2}} A_{F}^{2}(z, \lambda) e^{i \Delta k z}
\end{aligned}
$$

where $\Delta k=2 k_{\lambda}-k_{\lambda / 2}$ is the wave vector mismatch, $n_{\lambda}$ the index of refraction, and $d_{\text {eff }}$ the effective nonlinear susceptibility.

If we assume the undepleted pump approximation, we can use Eqs. (5.8) to compute the on-axis SHG for the intensity distribution of Eq. (5.5) by taking $A_{F}(z, \lambda) \cong \sqrt{I(r=0, z, \lambda)}$. In this case, the second equation of (5.8) can be integrated for each wavelength and the given crystal size $L$, and placing the crystal at different $z_{0}$ positions along the propagation axis we have:

$$
A_{S H}(L, \lambda)=\frac{4 \pi i d_{e f f}}{\lambda n_{\lambda / 2}} \int_{z_{0}-L / 2}^{z_{0}+L / 2} A_{F}^{2}(z, \lambda) e^{i \Delta k z} d z
$$

This simple formulation does not take into account the fact that all the wavelengths of the incident beam overlap in space and time, and therefore other wavelength mixes (SFG) could appear. That is, for a given wavelength $\lambda$ of the generated beam we need to take into account not

\footnotetext{
${ }^{13}$ This system of coupled wave equations can be obtained from those derived in Chapter 2 for SFG, (2.55)(2.57), assuming $A_{\mathrm{SH}}=A_{3}, A_{\mathrm{F}}=A_{1}=A_{2}$, and taking into account that $d_{e f f}(\mathrm{SFG})=d_{e f f}(\mathrm{SHG}) / 2$ and with $\omega_{3}=2 \omega$ and $\omega_{1}=\omega_{2}=\omega$.
} 
only the process $\frac{1}{\lambda}=\frac{1}{2 \lambda}+\frac{1}{2 \lambda}$ (SHG), but all the SFG processes, mixing wavelengths $\left(\lambda^{\prime}, \lambda^{\prime \prime}\right)$ such that $\frac{1}{\lambda}=\frac{1}{\lambda^{\prime}}+\frac{1}{\lambda^{\prime \prime}}$. Of course, the significance of each wavelength combination will be determined by the phase-matching conditions. These effects can be easily included in the calculations. Instead of Eq. (5.9), we have to integrate:

$$
A_{S F G}(L, \lambda)=\frac{4 \pi i d_{e f f}}{\lambda n_{\lambda}} \int_{z_{0}-L / 2}^{z_{0}+L / 2} A_{F}\left(z, \lambda^{\prime}\right) A_{F}\left(z, \lambda^{\prime \prime}\right) e^{i \Delta k z} d z
$$

for each pair $\left(\lambda^{\prime}, \lambda^{\prime \prime}\right)$ where now $\Delta k=k_{\lambda}-k_{\lambda^{\prime}}-k_{\lambda^{\prime \prime}}$.

The spectral maps depicted in Figure 5.6 represent the intensity of this field resolved spectrally:

$$
I_{S H}\left(z_{0}, \lambda\right)=\frac{1}{2} \sqrt{\frac{\varepsilon_{0}}{\mu_{0}}}\left|A_{S F G}(L, \lambda)\right|^{2}
$$

In order to compare the data with those obtained experimentally, we have taken a fundamental beam with central frequency $\lambda_{0}=795 \mathrm{~nm}$, and a Gaussian spectral shape with $9 \mathrm{~nm}$ at intensity FWHM.

If we look back at Figure 5.6 we see how the theoretical calculated maps match fairly well in the case of the 2-mm and $0.5-\mathrm{mm}$ thick crystals; i.e., the shape and the slope fits the calculations. However, they are not very accurate in the case of the thinner one. The experimental map is actually thicker than that calculated theoretically and this discrepancy can be explained in terms of the fact that in the model we did not take into account noncollinear interactions, either SFG or SHG. These are phenomena that may occur due to the use of tightly focused beams and the large angular acceptance of the thin crystal (See Figure 5.3).

\subsubsection{Efficiency of the Process}

Efficiency (external) was calculated as the measured energy of the second harmonic pulses divided by the energy of the incoming fundamental which was $44 \mathrm{~nJ}$. This value is a trade between having enough second-harmonic signal and avoiding damage to the crystal. The results are presented in Table 5.1.

\begin{tabular}{cccc}
\hline Lens & $\mathrm{E}_{\mathrm{SH}}(\mathrm{nJ})$ & Efficiency $(\%)$ & Crystal Thickness $(\mathrm{mm})$ \\
\hline Diffractive & 8 & $18(26)$ & 2 \\
Refractive & 13 & 29 & 2 \\
Diffractive & 4.5 & $10(14.8)$ & 0.5 \\
Refractive & 11 & 25 & 0.5
\end{tabular}

Table 5.1. Conversion efficiency measured with the refractive and the DL. In brackets, efficiency, taking into account the diffraction efficiency of the DL itself.

The conversion efficiency proves to be higher when focusing with the refractive lens for both crystals studied. The limited diffraction efficiency of the DL itself $(69 \%$ for the lens we used and calculated for the central wavelength of the pulse [76]) is the first phenomenon responsible for this result. If we calculate the conversion efficiency taking this factor into account, (the resulting values 
are in brackets in Table 5.1) the conversion efficiency approaches the values obtained with the refractive lens, mostly for the 2-mm thick crystal. However, for the $0.5-\mathrm{mm}$ thick crystal there still exists some discrepancy. This can be attributed to the strong chromatic aberration of the DL, which leads the energy of the input pulse to be distributed by diffraction along the optical axis. Therefore, it is not possible to convert all the spectral bandwidth of the fundamental into second harmonic for the same position of the crystal.

However, when working with focused beams we must make some new considerations in terms of efficiency. Back in 1968, Boyd and Kleinman [77] studied how to adjust the focus of the incident laser beam to optimize the efficiency of SHG. They found that the highest efficiency was obtained when the incident laser beam was focused such that the beam waist was located at the centre of the crystal and the ratio of the crystal length $L$ divided by the confocal parameter $b$ (also known as the Rayleigh length) $L / b$ was equal to 2.84 .

Under this assumption, the confocal parameter of the refractive lens (calculated for the central wavelength of the pulse and with a pulse radious of $4.5 \mathrm{~mm}$, at $1 / \mathrm{e}^{2}$ intensity criterion) would require a larger crystal thickness to optimize the efficiency $(\sim 700 \mu \mathrm{m})$ and, as stated, this would limit the converted second-harmonic spectra, with the consequent drawbacks. Therefore, in the case of the refractive lens the solution passes through choosing an adequate focusing length, (in this case it should be approximately $80 \mathrm{~mm}$ ) or increasing the input beam size.

In the case of the DL the option would be a new design of the lens. With this we would obtain two benefits: on the one hand, the diffraction efficiency could be optimized for the central wavelength of the laser and, on the other, for the central wavelength of the pulse we could choose the focal length that matched the condition before.

\subsection{Characterization of the Second Harmonic Generated with a DL}

Once we had demonstrated that it is possible to obtain tunability in the second harmonic generated with the DL we wanted to characterize it in order to propose our set-up as a robust and easy-tooperate system for tuning the second harmonic of the femtosecond laser pulses. This is the aim of this section, in which we shall describe the different measurements carried out to this end. We shall start with a temporal and spectral characterization of the pulses thus generated by using the XFROG technique. Then, we shall measure the beams' spatial chirp, making transversal scans with the spectrometer. And to conclude, we shall also measure the wave-front, with the help of a commercial wave-front sensor.

\subsubsection{Temporal and Spectral Characterization: X-FROG Measurements}

The X-FROG technique was already introduced in Chapter 3. Here we shall proceed in the same way and generate the third harmonic by means of the SFG process. The important issue is to incorporate a delay line in order to temporarily overlap the second harmonic and the fundamental pulses. Figure 5.7 shows a photograph of the experimental set-up, where the beams' paths have been drawn for clearness. From the laser output we incorporated a $(90 / 10)$ beam sampler (BSa). The sample, in reflection, is used for the SHG, passing through the lens and entering the first BBO $\left(\theta=29.4^{\circ}, \phi=90^{\circ}, 12 \times 12 \times 0.5 \mathrm{~mm}^{3}\right)$ where the second harmonic is generated (labelled "BBO-SHG" in the figure). We used the sample because since we were focusing on the crystal we did not need much energy. The other $90 \%$ is directed to the delay line, and its polarization is rotated. The two 
beams were again collected in a BBO sample $\left(6 \times 6 \times 0.1 \mathrm{~mm}^{3}\right)$ "BBO-SFG" to generate Type I SFG, $\left(\theta=44.3^{\circ}, \phi=90^{\circ}\right)$.

As on previous occasions, two dichroic mirrors separate the unconverted infrared from the blue signals generated. The position of these mirrors is related to the fact that we had to ensure that the second harmonic and fundamental beam sizes would be equal or at least similar before entering the BBO for SFG. Moreover the "BBO-SFG" sample was introduced in the place where the emerging divergent second-harmonic beam had the same size as the unfocused fundamental. Since the focal lengths used in both the diffractive and in the refractive cases were small, the beams showed considerable divergence, and this is why the distance between the mirrors had to be small in comparison with the rest of the set-up.

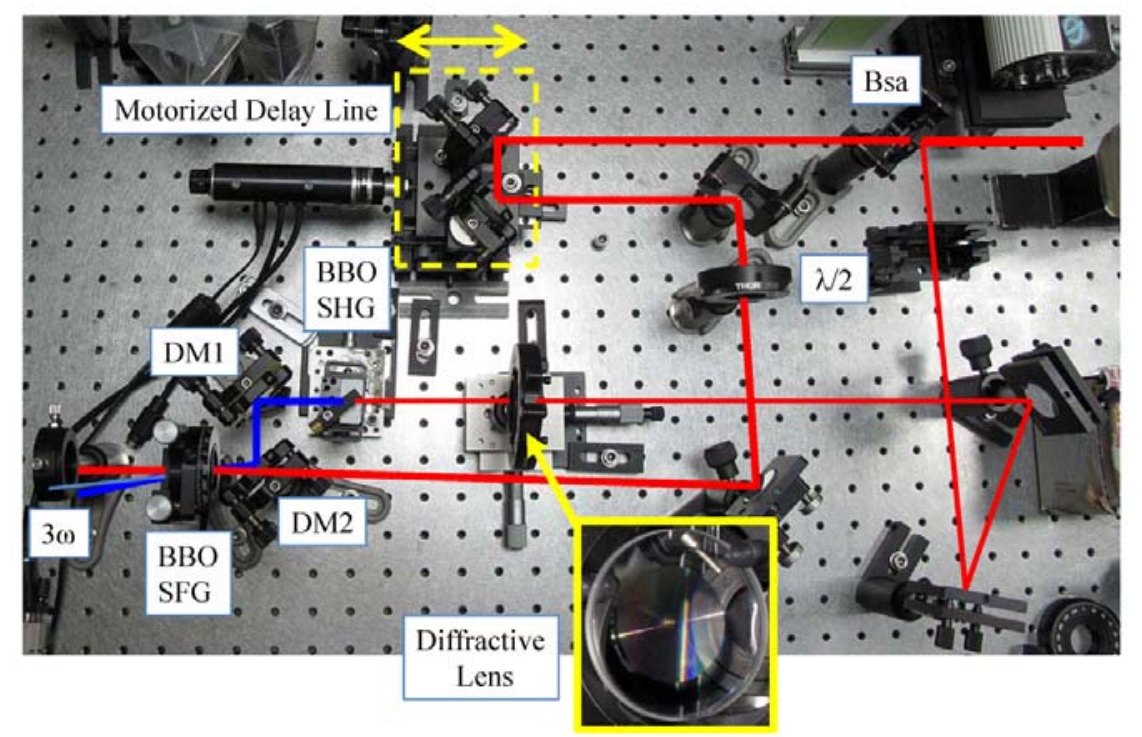

Figure 5.7. Picture of the experimental set-up for the characterization of the secondharmonic pulses generated with a DL in a BBO nonlinear crystal. BSa: 90/10 beam sampler. DM: Dichroic mirror.

In the following figure we show a sample trace obtained with the described set-up. We see that prior to the reconstruction of the pulse trace no additional chirp is introduced due to the focusing with the DL, because the trace is not tilted.

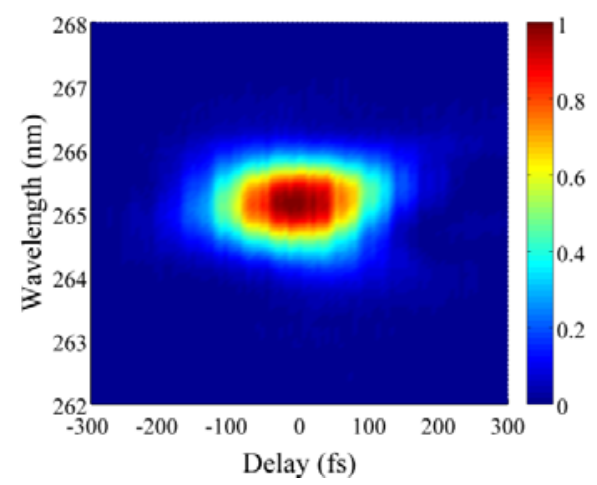

Figure 5.8. Experimental SFG-X-FROG trace for the characterization of second harmonicpulses generated in a 0.5-mm thickness BBO focusing the fundamental with a $D L$. 
As in the case of Chapter 3, we used the FROG algorithm (Frog 3, Femtosoft technologies [46]) in order to retrieve the temporal and spectral characteristics of the second-harmonic pulses. The results from the program are shown in the figure below.
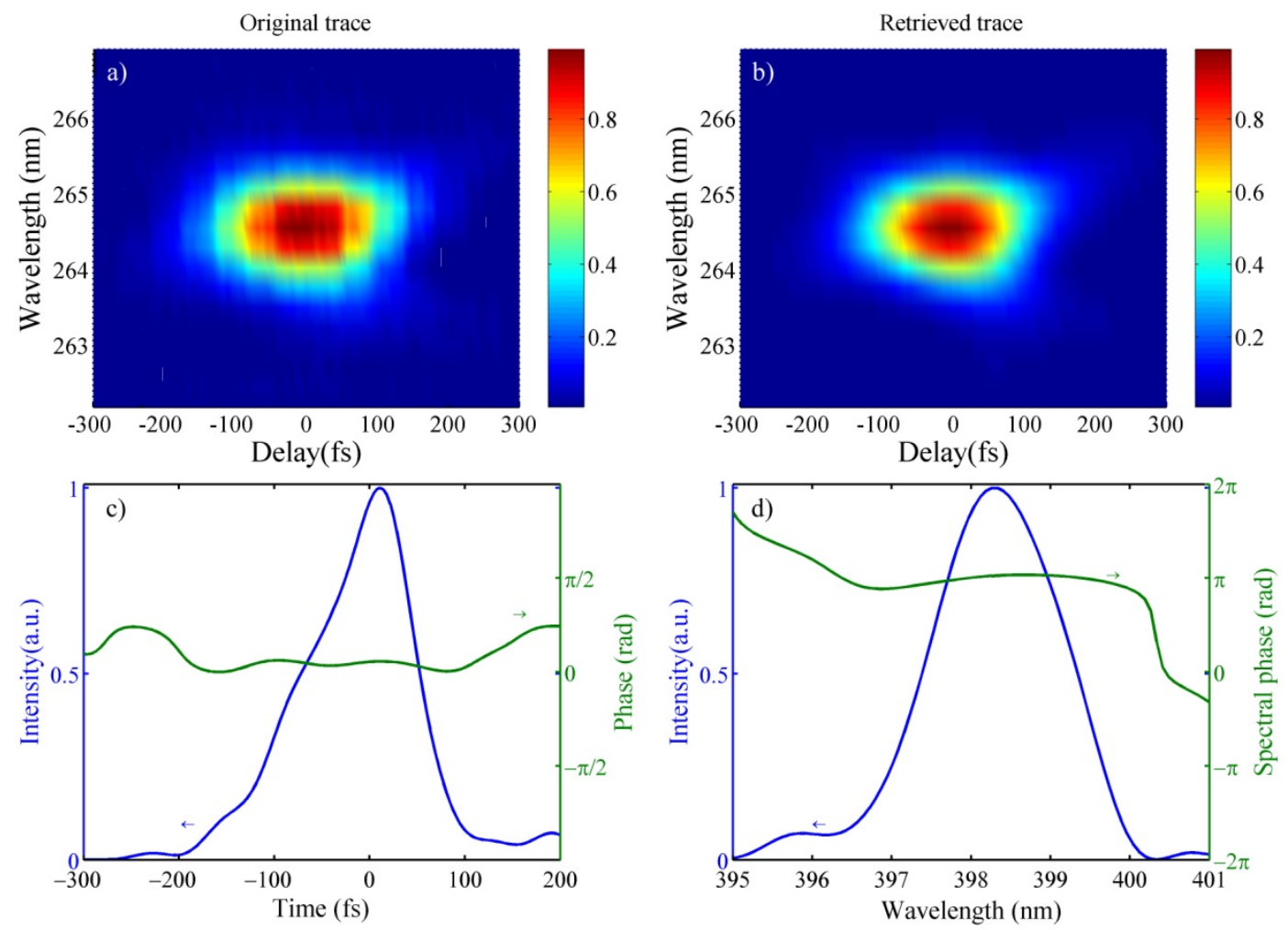

Figure 5.9. a) Experimental and b) retrieved X-FROG traces. The colourmap represents intensity in normalized arbitrary linear units. c) Reconstructed pulse intensity along with the temporal phase -green line-. d) Retrieved spectral intensity along with the spectral phase-green line-.

In this case, the results from the Frog suggest a pulse temporal duration of 125 fs (at intensity FWHM), with a time-bandwidth product of 0.46 . The best error in the retrieval was of the $0.7 \%$. However, although these results seem very good they should be taken with caution. In fact, there is a discrepancy in the spectrum reconstructed, which is broader than the measured one; $2 \mathrm{~nm}$ as compared with the $1.5 \mathrm{~nm}$ measured. Therefore, as a result the reconstruction gives much shorter pulses than expected. Since the temporal and spectral measurements (Sect. 5.3) were not taken on the same day, a small difference in the converted spectra is compatible with possible changes in the laser spectrum, or a miss-alignment of the nonlinear crystal.

As we did in Chapter 3, we can compare the Frog results with the data obtained from the crosscorrelation trace in Figure 5.10. This trace was obtained directly from the X-FROG trace. By simply integrating through all the wavelengths in each delay position, we can estimate the temporal duration of the SHG pulses [44]. Once we assume that we know the temporal duration of the fundamental pulse (130 fs, measured with the Grenouille as in the previous chapter) and with a duration of the trace of $200 \mathrm{fs}$, we can assess a duration of the second-harmonic pulses of approximately $150 \mathrm{fs}$ (considering Gaussian pulses). This result is in fairly good agreement with the transform-limited pulse duration calculated from the spectra in Section 5.3, although it is greater 
than the one given by the FROG algorithm. In our opinion, the real duration of the pulses might be somewhere in between these values: $125-150 \mathrm{fs}$.

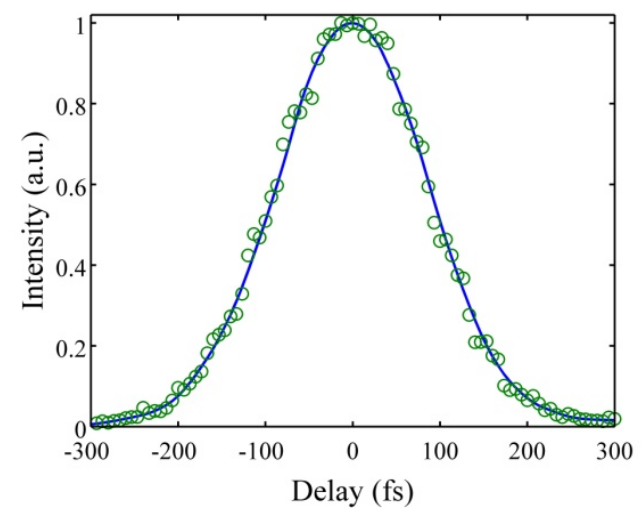

Figure 5.10. Cross-Correlation trace obtained from the time integration of the X-FROG trace.

\subsubsection{Spatial Chirp Characterization}

In this section we shall analyze the spatial distribution of wavelengths (namely spatial chirp) in the second-harmonic beams, generated with both the refractive lens and the DL, when focusing the IR pulses in the nonlinear crystal. Once again we used the $0.5-\mathrm{mm}$ thick BBO sample to generate the blue pulses. In order to make these measurements, we placed the optical fibre as indicated in Figure 5.11 and we recorded the spectra in different transversal positions of the second-harmonic beam, as indicated in the figure. We used the same spectrometer for the blue as before. The crystal was placed in the focus of both lenses, where the second harmonic signal was maximized. What can be expected is a spatial chirp, arising from the fact of the fundamental beam being focused.

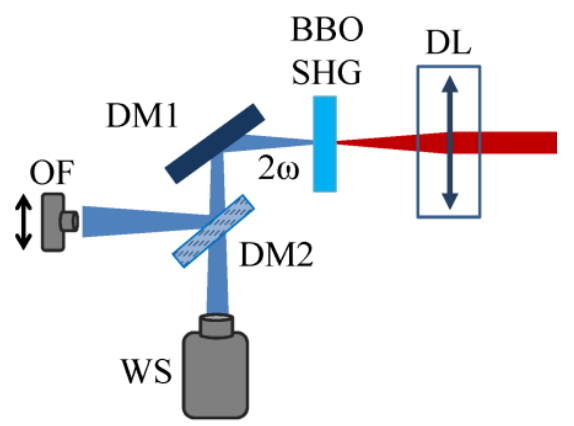

Figure 5.11. Diagram of the experimental set-up used to measure the spatial distribution of wavelengths and the wave-front of the second harmonic generated with a DL. DL: Diffractive lens, DM1,2: Dichroic mirrors, OF: Optical fibre, WS: Wave-front sensor.

In Figure 5.12 we plot the experimental $\lambda-\mathrm{x}$ maps taken with the refractive lens and the DL, at a distance $\mathrm{D}=13 \mathrm{~cm}$ from the crystal. In the vertical direction we plot the second harmonic spectra while the horizontal axis represents the fibre positions, $x$. If we compare the two maps, the most surprising effect is that the spatial chirp is reduced when focusing with the DL; in other words, the spectral content is almost constant in the transversal direction. In the figure we also include the integration over wavelengths in each position, $\mathrm{x}$. This helps us to see how the energy is distributed along the transversal direction. 
a)

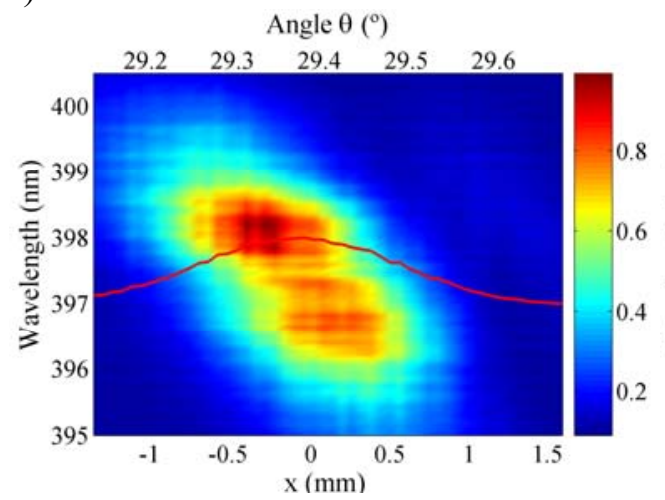

b)

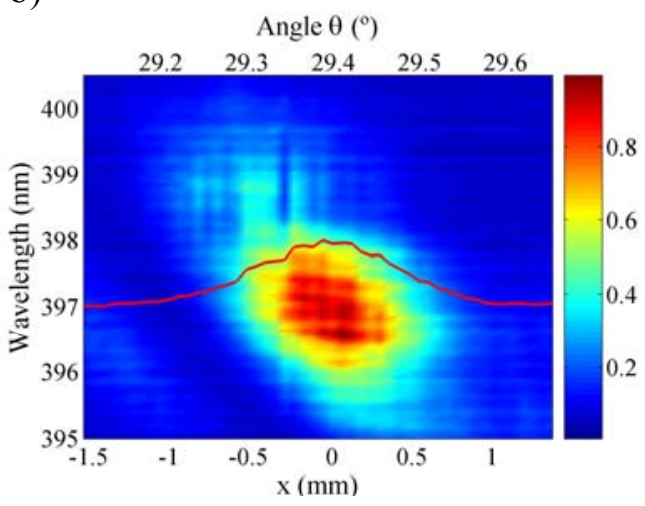

Figure 5.12. Experimental $\lambda-x$ maps for the second-harmonic beams generated with a) the refractive lens and $b$ ) the $D L$, focusing the IR pulses in a 0.5-mm thick BBO. The red line represents the integration in wavelengths over each position $x$. The colourbar represents normalized intensity in arbitrary linear units.

With this kind of measurement (the transversal maps) we should be able to extract information regarding the wave-front of the incoming beam (fundamental). This technique was developed by Borrego-Varillas [78]. However, in our experimental set-up that technique was not possible to apply because an imaging system should have been installed in order to image both the fundamental and the second harmonic beams at the output of the nonlinear crystal.

In a simpler approach we calculated the external angles (provided that we knew the distance and the increment in the transversal direction, $\mathrm{x}$ ) and consequently the internal angle, $\theta$, forming the direction of propagation with respect to the optical axis. These angles are plotted on the top axis of Figure 5.12, thus obtaining a $\lambda-\theta$ map.

The next step was to theoretically simulate these maps (see Figure 5.13). First, we calculated the phase-mismatch factor, Eq. (3.20), but taking into account that each wavelength was focused and therefore propagated at different angles, $\theta$, with respect to the c-axis inside the crystal. Then, we multiplied each map by the spectral intensity of the input beam to take into account the different amounts of energy travelling in each wavelength.

a)

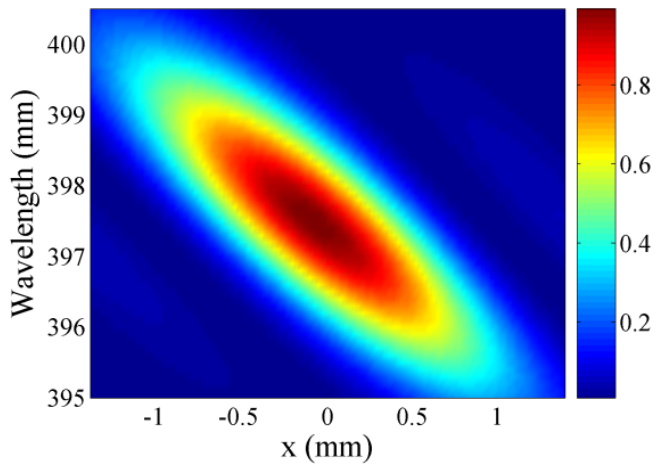

b)

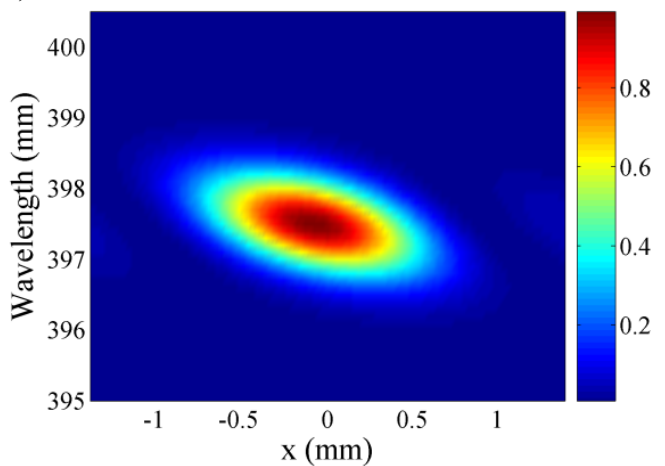

Figure 5.13. Theoretically calculated $\lambda$-x map in panel a) using the refractive lens to focus the beam on the BBO and in b) using the DL. The colourbar represents the efficiency in linear units. 
We see that the experimental maps for the two lenses match the theoretical calculation fairly well, at least in the tilting; typical of the focusing. However, the intensity distribution is slightly different. This is probably because we used a Gaussian pulse for the simulations, and to perform a more accurate calculation we should have measured the fundamental's spectral intensity reaching the sample and should have used this instead of the theoretical function.

In the case of the diffractive focusing, we see how the spatial chirp is reduced. This can be attributed to a narrower spectral intensity of the fundamental.

\subsubsection{Wave-Front Measurements}

In this section we used a wave-front sensor (SID4-HR, Phasics), which is a high-resolution multiwave shearing interferometer [79], to characterize the above beams. Placed at the same distance from the crystal as the fibre in the previous experiment, as shown in the Figure 5.11. Before explaining the wave-front measurements, we show images of the beams taken with the CCD camera of the sensor.
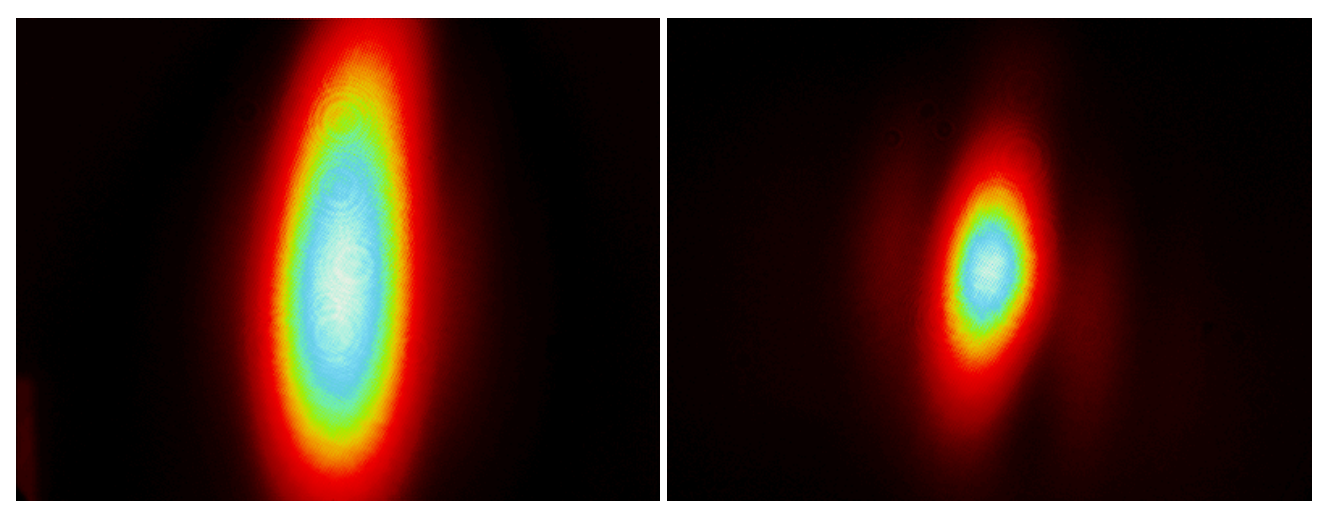

Figure 5.14. CCD camera image of the second harmonic generated with the achromat refractive doublet (left) and the $D L$ (right).

As can be seen in Figure 5.14 the signal no longer has a Gaussian spatial shape, and it exhibits astigmatism, generated by the angular acceptance of the crystal, which limits the efficiency, mainly in the horizontal direction, in the plane containing the optical axis, as argued in Sect 5.3. Note, however, that the effect is more important in the case of the refractive lens; since all the wavelengths are focused at the same time the beam is bigger and more intense and hence the difference in the horizontal and vertical size of the beam is more noticeable. In the case of the DL, the second-order maxima can be also seen at both sides of the more intense part.

\begin{tabular}{cccc}
\hline & Ellipticity & $\begin{array}{c}\text { Beam diameter X } \\
\text { a } 1 / \mathrm{e}^{2}\end{array}$ & $\begin{array}{c}\text { Beam diameter Y } \\
\text { @ } 1 / \mathrm{e}^{2}\end{array}$ \\
\hline Diffractive & 0.473 & 1.979 & 3.807 \\
Refractive & 0.319 & 3.035 & 9.343 \\
\hline & Table 5.2. Beam profile data given by the wave-front sensor.
\end{tabular}

Regarding the wave-front measurements (results can be seen in the Figure 5.15) we do not observe any difference in the wave-front of the second harmonic generated when the fundamental is focused with the DL or the refractive lens. However we must take this result carefully, owing to the retrieval method used for the wave-front sensor, which averages the wave-front of the different 
frequency components. It may happen that the sensor is "blind" to possible changes in the wavefront owing the strong chromatic aberration of the DL.

a)

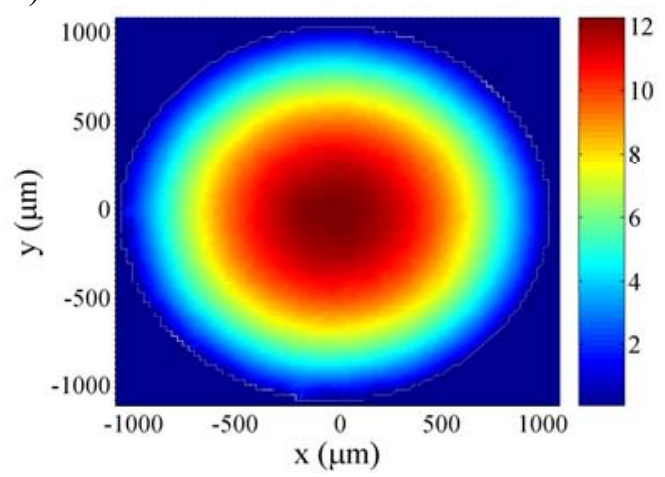

b)

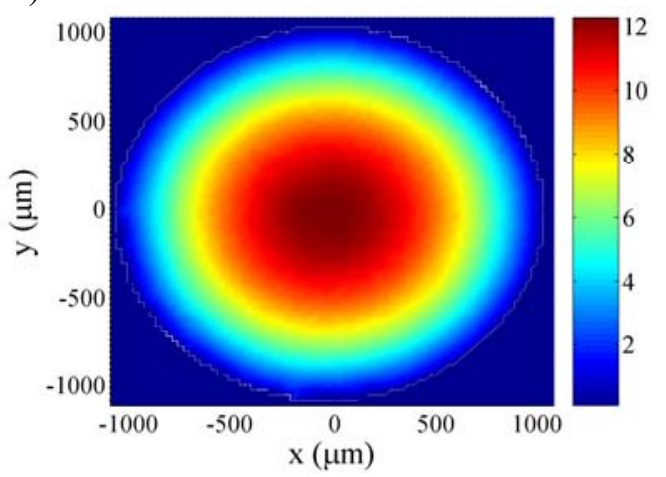

Figure 5.15. Wave-front measurements of the second harmonic generated with a) a refractive lens, b) a DL. The colour scale represents the wave-front variation over $\lambda$ in linear units $(\lambda=397.5 \mathrm{~nm})$.

\subsection{Wavelength Tunability in the Third-Harmonic Signal}

To conclude the chapter, in this section we shall show how the tunability obtained in the second harmonic can also be translated into the third-harmonic signal. The procedure bears some similarities with the characterization process because, once again, we shall obtain the third harmonic by means of the SFG process. Thus, the experimental set-up will be the same as for the X-FROG measurements (see Figure 5.7), although, with a difference in the location of the delay line. In this case, the computer-controlled motorized stage was coupled to the DL and a manual delay line was used to overlap the pulses temporally.

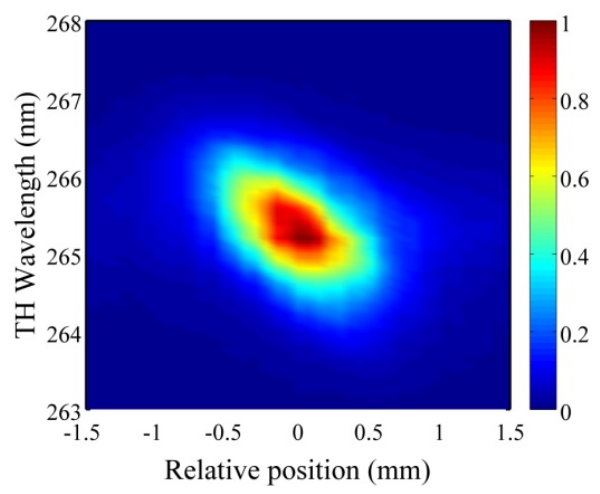

Figure 5.16. Third harmonic signal as a function of the relative lens-crystal position, $z$ when focusing the fundamental beam with a DL in BBO-SHG.

For this set of measurements we generated the second harmonic in a BBO of $0.5-\mathrm{mm}$ thickness (designated as "BBO SHG" in Figure 5.7), because this is the most suitable crystal length to observe tunability, as discussed before. The procedure for the measurement was as follows: first we chose a position for the DL in which we obtained enough signal in the second harmonic, (this must correspond to the doubling of the central wavelength of the laser), and then we changed the position of the delay line, for the second harmonic and fundamental pulses to be overlapped in the "BBOSFG" crystal, which was finely adjusted in order to maximize the third-harmonic signal. Following 
this, we changed the position of the DL that focuses the fundamental in "BBO-SHG", in the same way as we did for the measurements in Sect. 5.3.1; i.e., we recorded a spectrum in each lens-crystal position. A spectral map representative of these measurements is shown in the Figure 5.16.

As can be seen in the figure, the wavelength tunability is also observed, as for the SHG. In fact, the third harmonic signal can be tuned thanks to the wavelength selection induced in the second harmonic by means of the DL. This spans the tunability range of the device, although it is true that is not as easy to implement in the laboratory as for the second harmonic. However, it could be useful in spectroscopy to have a source in the near UV with some tunability.

In the case of the third-harmonic signal, the characterization is more challenging, mainly because of the low energy available. In addition, the autocorrelation is not possible, at least in BBO, because the phase-matching limit is in $410 \mathrm{~nm}$. Below this wavelength, SHG in BBO is not possible. One option could be cross-correlation with the fundamental, through the processes of DFG or SFG. It is possible to phase match the latter process and indeed the fourth harmonic of the Ti:Sapphire laser was obtained by this way in [80], although the resulting signal, $\sim 199 \mathrm{~nm}$, is at the limit of the transmission band of the BBO.

\subsection{Conclusions}

Here, we have demonstrated experimentally that a DL can be applied in the generation of secondorder nonlinear processes (SHG and THG) with femtosecond pulses, providing a compact and robust technique to achieve wavelength tunability in the signal thus generated by simply changing the lens-crystal distance. A complete study aimed at finding the best crystal size with respect to the lens used and the laser characteristics was also performed. An alternative technique to produce a similar tunability could be achieved by changing the orientation of a thick nonlinear crystal where the nonlinear process is taking place. However, this choice is not suitable for ultrashort pulses because the shifting of the phase-matching curve in relation to the pulse spectrum will lead to the generation of a modulated spectrum in the second-harmonic pulse and hence a degraded temporal shape.

Moreover, the second-harmonic pulses generated with the diffractive focusing were characterized, including temporal and spatial measurements, and revealed very good features. The temporal duration of the second-harmonic pulses is compatible with the clipped spectra that arise with the diffractive focusing, and the duration of the second-harmonic pulses remains in the fs regime, like the original (fundamental) ones. Moreover, the spatial shape of the beam reveals a decrease in the spatial chirp that arises when the fundamental beam is being focused. These favourable results open the possibility of applying these beams in other experiments: for example, in the field of spectroscopy. 


\section{Chapter 6}

\section{Supercontinuum Generation in Bulk Media with Diffractive Lenses}

\subsection{Introduction}

In this chapter, the strong chromatic aberration of the DL will be exploited to control the process of Supercontinuum (SC) generation. The latter is the spectral broadening that an ultraintense pulse undergoes when it propagates in a medium in which the dominant lowest order of the nonlinear susceptibility is $\chi^{(3)}$. The generation of new frequencies is in fact the result of the interplay of different phenomena, some of them non-parametric processes. In contrast with the second and third harmonic generation processes discussed in previous chapters, in the non-parametric case the initial and final quantum-mechanical states of the system are different. A typical SC signal covers the visible region of the electromagnetic spectrum from the near IR to the near UV. Our aim was not only to generate radiation in this region, but also to obtain improved features using the DL as a focusing element. Satisfactorily, new and unexpected effects appeared in the SC, which led us to perform a thorough investigation.

The chapter is organized as follows: first, we give a brief introduction of the SC generation process. Then we focus on SC generation in bulk media, in particular sapphire plates, and describe our experimental set-up. Concerning the experimental results, we first analyse the spectral characteristics of the continuum generated with the DL, including an analysis of the stability of the signal thus generated in terms of energy and wavelength fluctuations. Then we study the dependence on the parameters of the input beam, such as the NA for example. To conclude, the last 
set of experiments was directed to extending our results to other material thicknesses and other types of material. We end the chapter with our conclusions.

\subsection{SC Generation with Femtosecond Pulses}

As mentioned, SC generation occurs when a high-intensity (high-power) ultrashort pulse is focused into a transparent medium, as a result of the interplay of the different nonlinear effects that take place. The SC signal usually covers the whole visible range, as well as regions in the UV and in the near IR, thus appearing white to the eye. This is why the phenomenon is also termed white-light generation. SC generation was first discovered in 1970 by Alfano and Shapiro [81, 82] and since then it has been demonstrated in all kind of media: gases [83], transparent dielectrics (solids) [84] and liquids [85]. One surprising aspect of SC generation is that even though it was discovered more than forty years ago it is currently still not well understood. Particularly intriguing is the mechanism determining its spectral width. It is generally believed that several processes (both parametric and non-parametric) give rise to this exceptional broadband pulse; among others, self-phase modulation, self-focusing, self-steepening, plasma formation, four-wave mixing and filamentation. Although self-phase modulation is considered to be the dominant and triggering mechanism that leads to the above-mentioned spectral broadening, on its own it cannot explain certain complicated spectral characteristics. However, this uncertainty has not prevented the use of SC in a vast range of scientific disciplines. Among others, the applications of SC pulses are: femtosecond time-resolved spectroscopy [86], spectral interferometry [87], optical pulse compression for the generation of ultrashort pulses [88], and seed pulses for optical parametric amplifiers [89].

In our case the idea was to use the DL to generate SC in solids with the aim of exploiting it to seed an OPA (which will be described in the next chapter), but in the search for a stable continuum with improved qualities we found new interesting features that deserved detailed analysis [90]. Accordingly, we consider that a complete review of the processes involved in SC generation is necessary in order to give a hint as to the complexity of the process, and to help the reader to understand our experimental results and conclusions.

\subsubsection{The Optical Kerr Effect}

What is usually referred to as a Kerr medium is one in which the dominant lowest order of the nonlinear susceptibility is $\chi^{(3)}$. This is the case of the non-centrosymmetric media, such as gases, liquids or amorphous solids (glasses). In these cases, the index of refraction not only depends on wavelength but is also dependent on the intensity of the input beam in the following way:

$$
n=n_{0}+n_{2} I(r, t)
$$

where an instantaneous nonlinear response of the medium has been assumed. In the latter equation $n_{0}$ is the linear index of refraction, $I(r, t)$ is the external field intensity and $n_{2}$ is the so-called nonlinear index of diffraction, which must be determined experimentally [91, 92]:

$$
n_{2}=\frac{3}{2 n_{0}^{2} \varepsilon_{0} c} \chi^{(3)}
$$

This nonlinear index is a parameter that characterizes the strength of the optical nonlinearity. However, it is worth mentioning that this parameter $n_{2}$ is not the only important aspect to appreciate 
the nonlinear character of a medium. Note that the intensity of the pulse is also very important, because even in a medium with a low $n_{2}$ it would be possible to see the nonlinear effects, i.e., an intensity-dependent refractive index, if the pulse intensity $I(r, t)$ were sufficiently high. Currently, with the available high-power lasers it is not difficult to obtain the required intensities to see the nonlinear effects in a medium with a not very high $n_{2}$. Let us consider air, for example. With an $n_{2}$ $\left(5.57 \times 10^{-19} \mathrm{~cm}^{2} / \mathrm{W},[93]\right)$ three orders of magnitude lower than the typical values measured for condensed media $\left(3.25 \times 10^{-16} \mathrm{~cm}^{2} / \mathrm{W}\right.$ [94] in the case of fused silica, and $3.110^{-16} \mathrm{~cm}^{2} / \mathrm{W}$ [92] for sapphire), the effects of a nonlinear propagation can be seen when pulses of $0.2 \mathrm{TW}$ propagate freely in air. Thus, the output of such a powerful source must be conducted in a vacuum all along the way to the interaction chamber [95].

Self-focusing is a phenomenon that arises in the propagation of powerful laser beams in a Kerr medium. It is indeed the spatial manifestation of the optical Kerr effect and can be explained in the following way. Consider a Gaussian beam entering a medium of this type. Since the intensity of the beam is higher on the axis than at the borders, the index of refraction undergone by the beam will increase from the external parts to the centre (if $n_{2}$ is positive). This modulation of the index of refraction produces a modulation in the beam's wave-front similar to that of a convergent lens, with the difference that here the effect is cumulative and can lead, in the absence of other saturating effects, to a catastrophic collapse of the beam itself. This effect is represented diagrammatically in Figure 6.1.

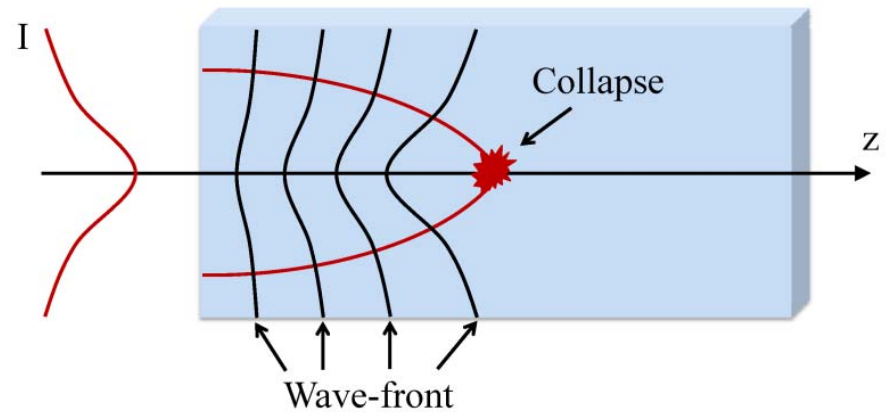

Figure 6.1. Diagram representing the process of self-focusing. When an ultraintense pulse enters a Kerr medium the wave-front is bent as if it the same pulse were entering a positive lens.

Self-focusing occurs in cases when the optical Kerr effect induced in the wave-front of the beam is large enough to overcome the linear diffraction of the beam. It can be demonstrated [9] that such a situation imposes a condition for the power of the beam: self-focusing (or catastrophic collapse) occurs when the beam power exceeds the so-called critical power, which proves to be:

$$
P_{\text {crit }}=\frac{3.77 \lambda_{0}^{2}}{8 \pi n_{0} n_{2}}
$$

where $\lambda_{0}$ is the laser wavelength.

After entering the medium, the pulse will collapse at a distance known as the self-focusing distance, which can be approximated fairly well by a semi-empirical formula [96]: 


$$
z_{f}=\frac{0.367 k a_{0}^{2}}{\left[\left(\sqrt{P / P_{c r i t}}-0.852\right)^{2}-0.0219\right]^{1 / 2}}
$$

where $P$ is the beam power, $k$ is the wave number, and $a_{0}$ is the input beam's radius at the $1 / e^{2}$ level of intensity. This distance is measured from the waist of the beam to the point where the selffocusing occurs. Although in the majority of cases the beams are focused with lenses, one must calculate the equivalent $z_{f}^{\prime}$ as:

$$
z_{f}^{\prime}=\frac{z_{f} f}{z_{f}+f}
$$

This means that with a positive lens, $z_{f}^{\prime} \leq f$, self-focusing occurs before the geometrical focus of the lens.

In condensed media, the critical power for self-focusing is in the range of MW, while in the case of gases it is in the range of GW. This implies that with only $1 \mu \mathrm{J}$ of energy and for a laser pulse of $100 \mathrm{fs}$ we can obtain self-focusing in a solid. Therefore, to study this phenomenon there is no need to have a very powerful laser, such as the one we mentioned before, and a regeneratively amplified Ti:Sapphire system is sufficient.

\subsubsection{Self-Phase Modulation}

Self-phase modulation is the temporal manifestation of the optical Kerr effect. The temporal variation of the laser intensity for a pulse involves a temporal variation of the refraction index as reflected in Eq. (6.1), which in turns corresponds to the appearance of new frequencies in the spectrum of the laser pulse. The simplest model accounting for the evolution of the temporal phase links the instantaneous frequencies to the pulse intensity as:

$$
\omega(t)=-\frac{\partial \phi}{\partial t} \approx \omega_{0}-\frac{n_{2} \omega_{0}}{c} z \frac{\partial I(r, t)}{\partial t}
$$

The generation of new frequencies thus depends on the slope of the pulse, $\partial I(r, t) / \partial t$, the propagation distance $\mathrm{z}$ in the medium, and its nonlinear index coefficient, $n_{2}$. It is clear from this equation that for the same pulse intensity the shorter the pulse the greater the frequency modulation. In a purely Kerr medium, the front part of the pulse generates redder frequencies i.e. lower frequencies that the central wavelength of the pulse (positive slope), and the back part generates bluer frequencies (negative slope). If the pulse shape is initially Gaussian or nearly Gaussian, the spectrum of the pulse after undergoing self-phase modulation becomes symmetrically broadened. A typical spectrum broadened by self-phase modulation can be seen in Figure 6.4 -light green line-. This effect plays a significant role in SC generation with femtosecond pulses.

\subsubsection{Self-Steepening}

Laser pulse self-steepening occurs because the group velocity of the peak intense part of the pulse is smaller than that of the trailing edge, due to the intensity-dependent change of the refractive index $\Delta n=n_{2} I(r, t)$. For $\Delta n$ positive and instantaneous, the light velocity decreases with increasing 
laser intensity. Therefore, in the propagation of a Gaussian pulse the peak will be slowed down with respect to the pulse group velocity, while the trailing part will catch up with the peak. This effect leads to the formation of a steep edge in the trailing part of the pulse. In fact, the energy is shifted to longer times. In other words, the input pulse will undergo an asymmetric breakup into a weak leading part and a strong self-focused trailing pulse. Also, a shock wave is formed in its trailing edge. This effect leads to a faster self-focusing of the trailing part of the pulse (in comparison with the leading part), while the leading part is suppressed, forming only a pedestal. Moreover, the steep trailing part leads to an increase in the bluer frequencies because of a higher slope: $\partial I(r, t) / \partial t[97]$.

\subsubsection{Ionization}

All the processes described above are originated by the third-order susceptibility of a medium. They correspond to the group of parametric processes provided that the initial and final quantummechanical states of matter are the same. However, as the intensity of a laser pulse increases, the ionization of the medium (a non-parametric process) will also play a role. Ionization, or plasma generation, consists of the generation of free electrons that are released from the bounding potentials that keep them linked to their "parent" ions. Intense ultrashort pulses can generate free electrons through several mechanisms that are generically termed as Strong Field Ionization. Depending on the laser parameters (mainly the intensity), the ionization can be produced by Multiphoton Ionization, Tunnel Ionization, or Barrier Suppression. Once some electrons have been released by one or several of these mechanisms, the acceleration of the electrons and recollision against other neutral atoms leads to the generation of more released electrons, in a process that is called Avalanche Ionization [98].

a)

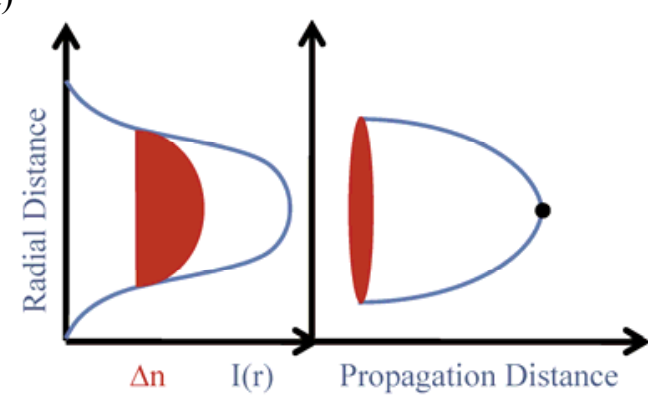

b)

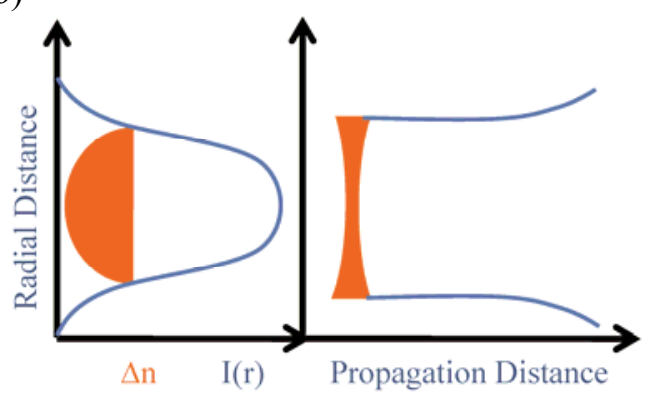

Figure 6.2. a) Self-focusing of a beam due to the optical Kerr effect. The refractive index of the medium depends on the intensity of the laser and acts as a positive lens by making an initially collimated beam convergent. b) Defocusing of the beam in the presence of plasma. The creation of an under-dense plasma decreases the local index of the medium, which causes beam defocusing.

The generation of plasma by the laser pulse has several consequences as regards its propagation. On the one hand there will be absorption due to the photons absorbed by the electrons to be ionized. On the other hand, the plasma itself will modify the index of refraction. If we assume that the plasma generated has low density, the refractive index is modified as follows:

$$
n=n_{0}-\frac{\rho(r, t)}{2 \rho_{c}}
$$


where $\rho(r, t)$ is the density of free electrons and $\rho_{\mathrm{c}} \equiv \varepsilon_{0} m_{0} \omega_{0}{ }^{2} / e^{2}$ is the critical plasma density, above which the plasma becomes opaque. The low-density condition means that $\rho \ll \rho_{\mathrm{c}}$. As can be seen from this equation, a low plasma density decreases the index of refraction. This effect is opposed to that created by the optical Kerr effect, and it tends to defocus the beam. The ionized medium acts as a divergent lens because the plasma density will be larger at the centre of the beam than at the borders. See Figure 6.2 b).

\subsubsection{Filamentation}

Filamentation is the creation of a light channel (filament), with negligible divergence, over a distance much larger than that expected by the limits of diffraction. The filament is created due to the interplay of the different nonlinear processes described in this section. When a laser pulse (with power above $P_{\text {crit }}$ ) propagates in a Kerr medium, it will undergo self-focusing. As the pulse converges towards the nonlinear focus, the intensity at the centre of the beam will increase enormously. When the intensity is large enough to ionize the medium, a free-electron plasma will be created with larger density close to the axis. This plasma will produce a decrease in the refractive index that will tend to defocus the beam. Under certain conditions, the self-focusing effect of $\chi^{(3)}$ can be compensated with the defocusing effect of the plasma (and diffraction), leading to a situation of equilibrium where the light propagates with no appreciable change in beam size. The collapse predicted by the optical Kerr effect is thus prevented by both linear refraction and plasma divergence.

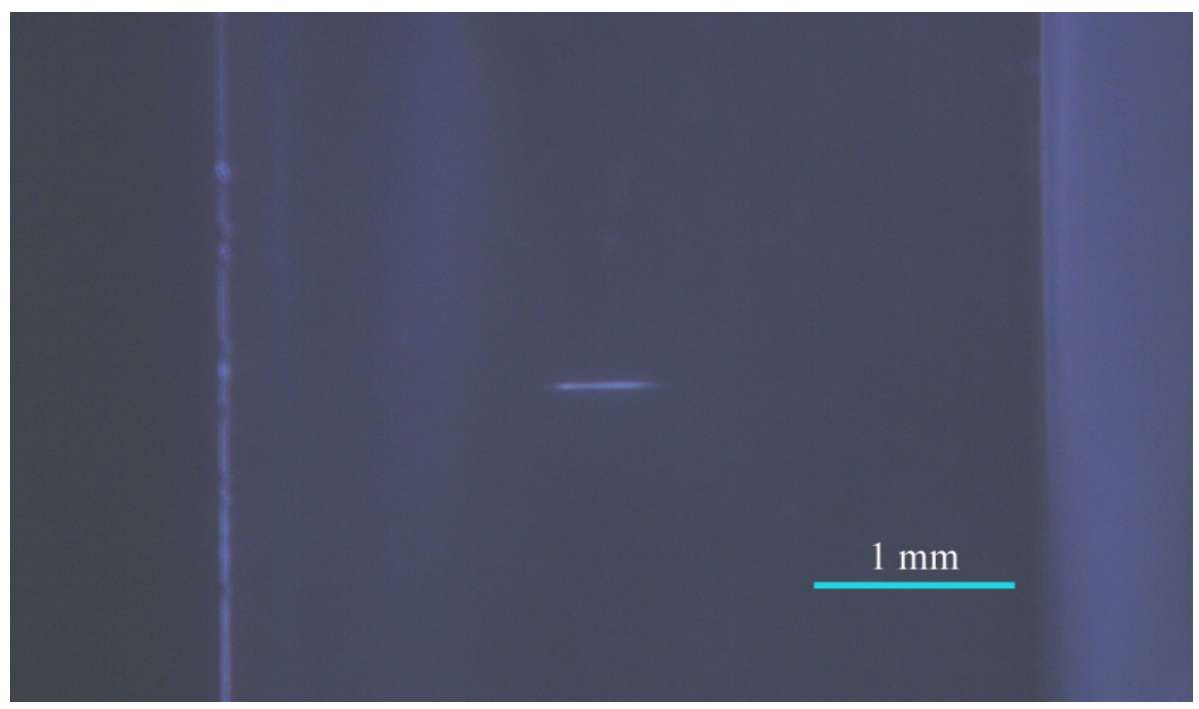

Figure 6.3. Image of a filament inside a 4-mm thick fused silica block. What we actually see is the relaxation of the plasma (inverse Bremsstrahlung), corresponding to the emission of photons with wavelengths that fall in the visible part of the spectrum.

Finally, therefore, filament formation is a compromise between the self-focusing induced by the intensity of the pulse and the divergence of the pulse due to the above-mentioned effects of diffraction and plasma defocusing. A very complete review of the process of filamentation and other complex phenomena related to SC generation is offered in reference [99]. An example of a filament inside a fused silica block is shown in Figure 6.3. Approximately $2 \mu \mathrm{J}$ of the laser energy was focused, with a $100 \mathrm{~mm}$ focal length lens inside a 4-mm thick block to generate this filament. 


\subsubsection{Spectral Broadening and SC Generation}

SC generation results from an intense ultrashort pulse undergoing the nonlinear processes described above. Most authors agree that self-focusing is the triggering process that gives rise to the appearance of the SC [84]. In fact, it was found experimentally that the calculated critical power for self-focusing turns out to be the same as the power threshold for white-light generation, supporting the hypothesis that SC generation is triggered by self-focusing. (See [100] and references therein). It is considered that the onset of catastrophic self-focusing leads to a drastic increase in intensity, which further enhances self-phase modulation. Catastrophic self-focusing is stopped by the plasma generated due to the high intensity reached near the nonlinear focus. The plasma is also essential for $\mathrm{SC}$ generation because it contributes to anti-Stokes (blue shifted) spectral broadening. Additionally, other nonlinear effects such as four-wave mixing or conical emission also contribute to the spectral reshaping of the pulse. Consequently, an initial pulse with its spectrum centred in $800 \mathrm{~nm}$ (near infrared) would be partially converted into a continuum, covering the entire visible part of the electromagnetic spectrum when it propagates inside a nonlinear media, particularly in solids. As a result of the broad spectral content, the beam appears white to the naked eye when it is projected onto a screen. In Figure 6.4 a typical SC spectrum can be seen: the brown line. In this case, it was generated in 3-mm thick sapphire plate and is shown along with a spectrum of the Ti:Sapphire laser: the dark green line. Typical features of the continuum that can be recognized in the figure are a modulation near the laser wavelength; smoothness in the wings, and a long tail in the blue part of the spectrum. In general, the SC generated in bulk media exhibits asymmetry, in the sense that the new wavelengths are generated in the visible rather than in the infrared part of the spectrum; i.e. the $\mathrm{SC}$ is not symmetric with respect to the wavelength of the initial pulse.

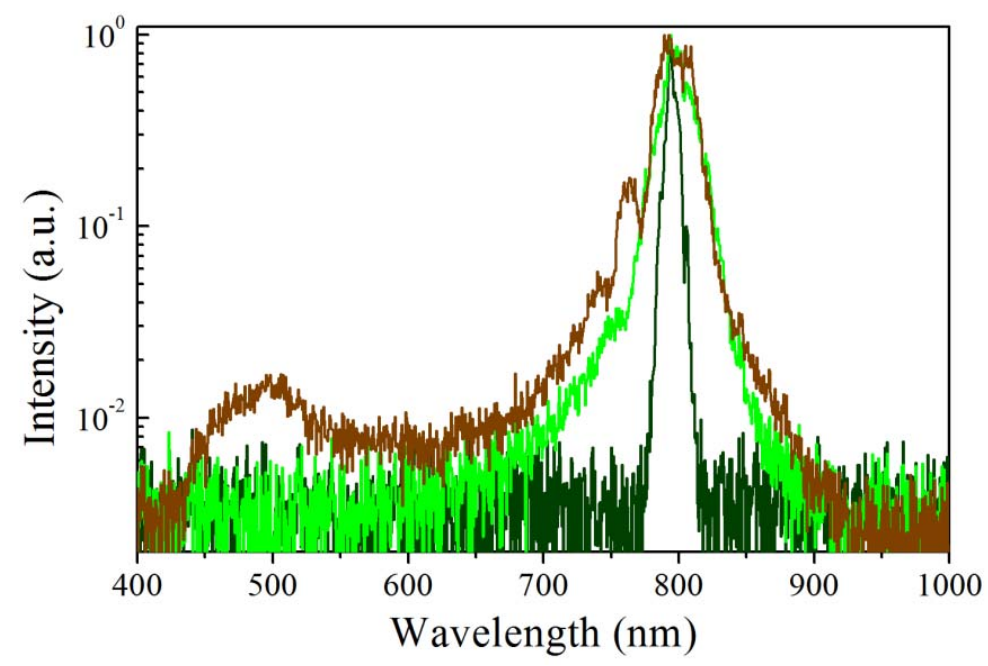

Figure 6.4. SC spectrum generated in a 3-mm thick sapphire sample -brown line-, spectrum of self phase modulation -green line- and spectrum of the initial laser pulse, -dark green line-.

It should be noted that the fibre position was not the same to record the spectra shown in Figure 6.4 because we had to move it in order to avoid the intense IR peak when recording the SC. Therefore, we take as a reference only the spectral content and not the relative intensity of one spectrum with respect to the other. 
In our case the extent of the spectral broadening is one of the most important features; this can help us to select one material from another. Several effects combine to cause spectral broadening: self-phase modulation, self-steepening and ionization of the medium, which mainly add new frequencies on the high-energy side of the spectrum (blue edge). One of the determining factors for the extension of spectral broadening is the band-gap of the medium [101]. It was found that bluebroadening scales with band-gap, i.e. the anti-Stokes broadening increases with increasing $\mathrm{E}_{\mathrm{gap}}$ and there is also a threshold for $\mathrm{SC}$ generation that requires an $\mathrm{E}_{\text {gap }}$ of the material greater than $4.7 \mathrm{eV}$. If we compare the two materials that we used for $\mathrm{SC}$ generation -fused silica with $\mathrm{E}_{\text {gap }}=7.5$ and sapphire, with $\mathrm{E}_{\mathrm{gap}}=6.5$ [102]- we shall see later in the experiments how the extension of the blue edge in fused silica is lower than in the sapphire.

In the previous chapter we applied a DL to the parametric processes of SHG and THG. Here we shall use it to focus the femtosecond laser on sapphire or fused silica plates. As stated in the previous chapter, the kinoform lens (with a focal length $f_{0}=106.6 \mathrm{~mm}$ for $\lambda_{0}=795 \mathrm{~nm}$ ) had a strong chromatic aberration Eq. (5.2), which led to an extended focal region of $1.3 \mathrm{~cm}$ when $9 \mathrm{~nm}$ intensity FWHM bandwidth pulses were focused on it. Previously, diffraction had been applied for the generation of SC [103], and in that case an iris was used to redistribute the intensity along the propagation axis to produce a stable SC. However, to our knowledge a DL has not been applied before.

\subsection{Experimental Arrangement for the Generation of SC with a DL}

In the case presented here, we generated $\mathrm{SC}$ in two transparent dielectric materials, fused silica $\left(\mathrm{SiO}_{2}\right)$ and sapphire $\left(\mathrm{Al}_{2} \mathrm{O}_{3}\right)$, although the majority of our measurements were performed in sapphire. The main consideration related to the preference of one material over the other was robustness in the operation time. From our previous experience in $\mathrm{SC}$ generation with refractive lenses, we were aware that the SC generated in sapphire was stable for longer than the one generated with fused silica under the same experimental conditions. This observation has already been reported in other works $[89,90]$.

The experimental set-up for the SC generation consisted of four key elements: the lens, the sample, an energy control system and the detection system. See Figure $6.5 \mathrm{~b}$ ). As mentioned before, in this set of experiments we used two different materials in which we generated the continuum. One was a glass (fused silica) and the other a crystal (sapphire). In fact, the work was mainly focused on sapphire and the glass was only used in order to test our results in other material.

In the case of sapphire, because it is a birefringent crystal we have to take into account that it can act as a retarder wave plate for the input pulse, changing its polarization state. This is something that in principle does not occur during the process of the $\mathrm{SC}$ formation. In principle, if the input pulse is linearly polarized the SC signal will maintain the same polarization state [104]. Furthermore, bearing in mind that our intention was to use this continuum as a seeding of the OPA, we needed it to be linearly polarized in order to maintain certain phase-matching conditions. In the particular case of parametric amplification in the visible, the signal must be polarized orthogonal to the pump. Therefore, if part of the signal (the SC) has a change in polarization it will not be amplified, with the consequent loss in efficiency.

We used sapphire plates with their optical axis content at the entrance plane of the sample, called the z-cut, but with random orientation. This meant that we had to orientate the sapphire to set 
the optical axis either orthogonal or parallel to the laser polarization. The way to do this is to place the sample between two linear polarizers with their transmission axes crossed. Since our samples were round, this can be seen in Figure 6.5. We placed them in a rotating mount, so we could rotate them until we did not see any light at the output. We used an He-Ne laser for this experiment because it is safer and easier to use than the ultraintense one.

Moreover, it is very important to have precise control over the incident pulse energy for the experiment. As we shall see in detail later, different effects appear around energies per pulse of 1 to $2 \mu \mathrm{J}$. Usually, the resolution of the power meter is around $1 \mu \mathrm{J}$, and hence it is very important to find a way to make more precise measurements. Our solution was to interpose a set of neutral density filters, previously calibrated, in front of the lens, and measure the energy reaching the filters, which was in the order of 10-20 $\mu \mathrm{J}$. Then we applied the corresponding transmission factor to calculate the energy reaching the sample. With this we reduced the absolute error in the power meter measurement. To finely control the input energy we used a half-wave plate and a linear polarizer, as done in previous experiments.

a)

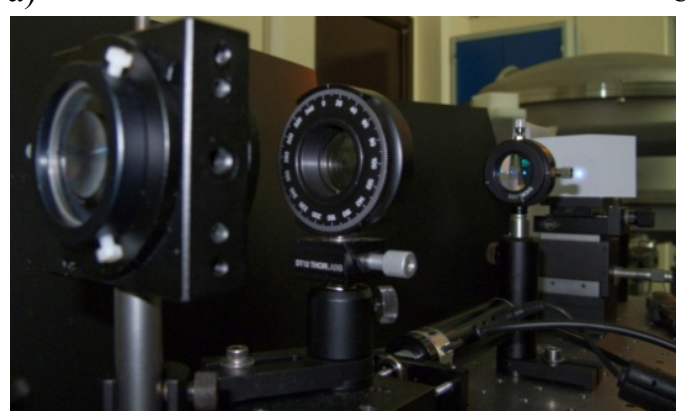

b)

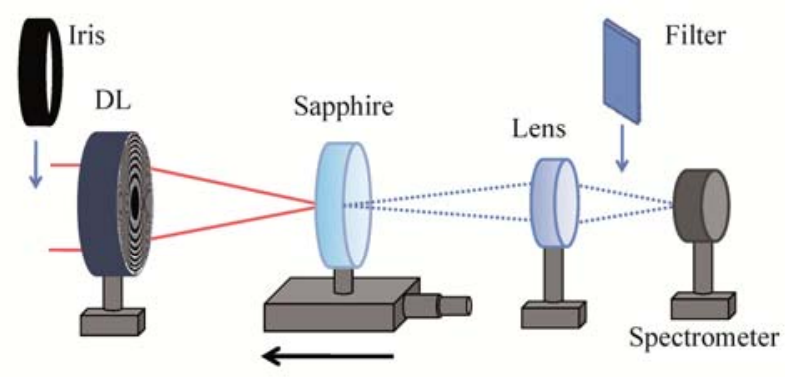

Figure 6.5. a) Photograph and b) sketch of the experimental set-up for SC generation with a kinoform $D L$.

As can be seen in the diagram of Figure $6.5 \mathrm{~b}$ ), an iris can be interposed before the lens in some sets of experiments in order to reduce the NA by reducing the beam size. After the lens, we placed the sample, which was mounted on a motorized delay line, allowing us to control the lens-crystal distance precisely. Although this was not mandatory to perform the experiments, it was really convenient because it allowed the possibility of making systematic measurements in a very short time. After the sapphire we collected the light with a fused silica biconvex lens, to help to couple it into the optical fibre and reduce possible transverse spatial dependence of the SC spectra. The optical fibre was placed some millimetres away from the focus of the biconvex lens. This position was chosen as a solution to collect most of the SC signal and in an attempt to minimize the effect of the chromatic aberration introduced by this lens.

In order to prevent the fibre from saturation, a band-pass filter was interposed before the fibre to block the unconverted IR light (FGB37s, Thorlabs). The spectra recorded were corrected with the optical transmittance of the filter. See Appendix B for further details. Spectral analysis of the SC pulses in the visible region was done with a spectrometer (Avantes, AvaSpec-2048) coupled to a 50 $\mu \mathrm{m}$-core optical fibre (Ocean Optics, P50-2-UV/VIS). 


\subsection{SC Generation in Sapphire. Experimental Measurements and Analysis of the Results}

SC generation is a phenomenon that depends on several parameters of the input beam that are somehow connected. For example, it depends on the NA, which depends on the beam's diameter and the focal length of the focusing lens. The SC also depends on the intensity and on the central wavelength of the pulses. Therefore, in order to study experimental situations that were comparable we calculated the thresholds for SC generation for the different materials and thicknesses used in the experiments when focusing the IR pulses with the DL. We started from a very low energy value, after which we increased it progressively until we saw the white light projected on a white screen. The incident pulse energy was measured before the neutral density filter. Then, to determine the pulse energy reaching the sample, the transmittance of the filter and the energy losses due to the diffraction efficiency of the DL were taken into account, see Sect. 5.3.3. Our results are summarized in Table 6.1. The values marked with an asterisk represent the thresholds when we focused the beam with a refractive lens, with a focal length of $100 \mathrm{~mm}$ (Linos, G063144525). As in the previous chapter, this lens was chosen to maintain the same NA of the DL.

\begin{tabular}{|c|c|c|c|c|}
\hline & \multicolumn{3}{|c|}{ Sapphire } & Fused silica \\
\hline Thickness & $3 \mathrm{~mm}$ & $2 \mathrm{~mm}$ & $1 \mathrm{~mm}$ & $3 \mathrm{~mm}$ \\
\hline Pulce & $0.97 \mu \mathrm{J}$ & $0.97 \mu \mathrm{J}$ & $1.66 \mu \mathrm{J}$ & $1.24 \mu \mathrm{J}$ \\
\hline energy & $0.46 \mu \mathrm{J}^{*}$ & - & - & $0.50 \mu \mathrm{J}^{*}$ \\
\hline
\end{tabular}

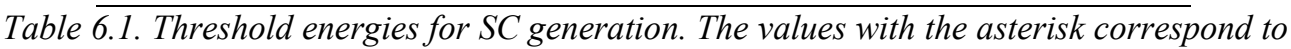
focusing the IR with the refractive lens.

The energy threshold is larger (around two times) for the DL lens than that measured for the refractive lens. This can be explained in terms of the diffraction introduced by the DL, producing a readjustment of the energy in the focal region and thus decreasing the intensity at the focus. See Figure 5.2.

Note that the $1 \mathrm{~mm}$ sapphire plate provided the largest threshold. We explain this effect in terms of the notion that the focal region of the DL $(1.3 \mathrm{~mm})$ is larger than the crystal thickness. Thus, in this case the whole focal region does not fit completely inside the crystal and consequently part of the pulse energy cannot be converted into SC light.

It is well known that the NA of the incident pulse plays a fundamental role in SC generation [100]. As reported in [102], there exists an optimum NA that significantly improves the stability and the spectral extension of the pulses generated and it can be set by using an iris placed in front of the focusing lens. Following this idea, we used an iris as a control element. Then, we considered that it was appropriate to determine the thresholds for SC generation in terms of the iris diameter. To this end, for each iris size and pulse energy tandem a scan of the 3-mm thick sapphire sample around the focal region of the lens was performed, simply to ensure that no SC emission would appear at any position. The incident pulse energy was measured before the neutral density filter for the reasons given before. To determine the pulse energy reaching the sample, a previous calibration was done, measuring the energy transmitted through the different iris diameters and the filter. The losses in the DL (mainly due to the diffraction efficiency, as we stated before) were also taken into account. The results are shown in Figure 6.6, where we also show the thresholds measured when the DL was replaced by the achromatic doublet for comparative purposes. As expected from the 
longer focal volume of the DL, the threshold for all the iris sizes was larger (around two times) than that of the achromatic lens. As stated in the introduction of the chapter, we needed to ionize the medium since the SC is an effect in which intensity is a very important parameter and is reduced if the focus of the lens is spatially distributed. Therefore, it is not surprising that more energy is needed when focusing with the DL to generate the SC.

Whenever SC light was generated, a bright white spot was visible inside the sample, suggesting the formation of a filament. A minimum value was observed at an iris diameter of $4 \mathrm{~mm}$ that appeared for both lenses. We have no conclusive explanation for this but do suggest that it could arise from a better fitting of the energy distribution at the focus, due to diffraction, with the selfguided mode in the filament.

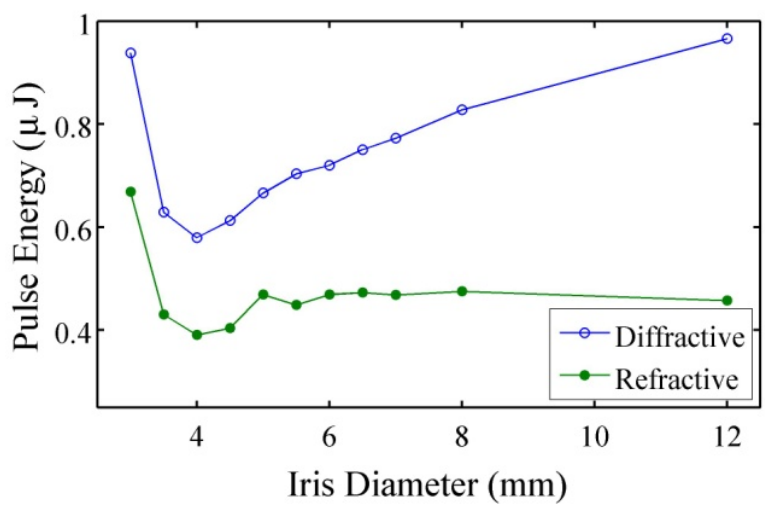

Figure 6.6. Threshold energies for SC generation in sapphire when focusing with the DL in terms of the iris diameter. Threshold measurements for a refractive lens are also given for comparison.

With this is mind, we now address the experimental measurements and results obtained with the 3 -mm thick sample. First, we set the energy of the input pulses to $1.38 \mu \mathrm{J}$ (this value is approximately 1.4 times the generation threshold for these conditions, which involved focusing with the DL without an iris). The crystal was moved in direction towards the lens in steps of $50 \mu \mathrm{m}$, as indicated with an arrow in Figure $6.5 \mathrm{~b}$ ), thereby reducing the lens-sample distance. The motorized stage (see Figure $6.5 \mathrm{~b}$ )) was connected to the spectrometer through a Labview program, which allowed us to take a spectrum at each position of the motor. Then, the spectra were corrected with the transmittance of the filter, as already mentioned (included in Appendix B).

The results are shown in Figure 6.7, where the wavelengths are represented on the vertical axis, and the relative positions, $\mathrm{z}$, are shown on the horizontal one. The value $\mathrm{z}=0$, corresponds to the largest distance between the lens and the crystal, also corresponding approximately to the position where the geometric focus of the lens is at the entrance face of the sapphire plate and, as $z$ is increased, the focus is moved to the rear (output) face of the sample. This is considered to be detrimental to the formation of the filament and at these points the stability of the continuum was poorer. For the sake of comparison, we present the same map, taken under equivalent experimental conditions but with the refractive lens. 

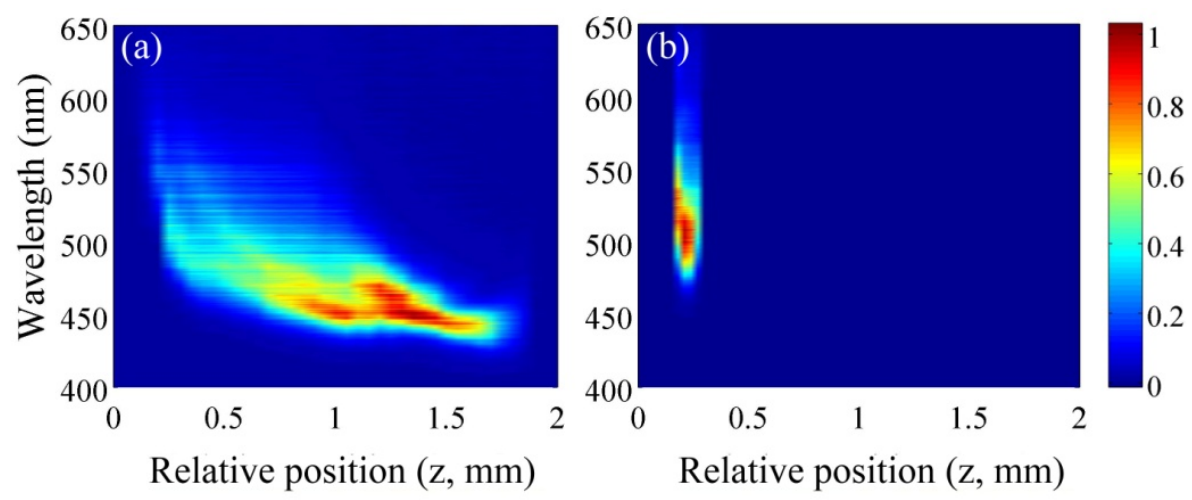

Figure 6.7. Spectral maps taken when focusing the fundamental a) with the $D L$ and b) with a conventional refractive lens with the same focal length. The colour scale represents normalized intensity in arbitrary linear units.

The differences between the two maps are clear. First of all, the extension of the region where the SC is generated is larger in the case of the DL, and secondly, the peaked structure observed in the case of the DL is not present in the map taken with the refractive lens. To clarify this point, in Figure 6.8 we show some spectra extracted from the map in Figure 6.7 a). In this figure, a new characteristic of the SC can be observed: the central wavelength of the peak changes when changing the relative distance between the lens and the crystal, z. It can be seen that as the sample approaches the lens (a higher value of $\mathrm{z}$ ), the central wavelength of the spectra is shifted down in wavelengths. Therefore, by changing the relative distance between the crystal and the DL the spectral features of the SC can be controlled easily.

These are two new characteristics of the continuum generated with this lens, which are not observed when focusing with a conventional refractive lens. Moreover, the greater extension of the spectral blue border as the focus is moved to the rear face of the crystal (decreasing the lens-crystal distance) is opposite to that previously described in several works in which an achromatic focusing element was used. In those cases, the maximum extension of SC was achieved when the focus was near the front face (see for instance reference [105]).

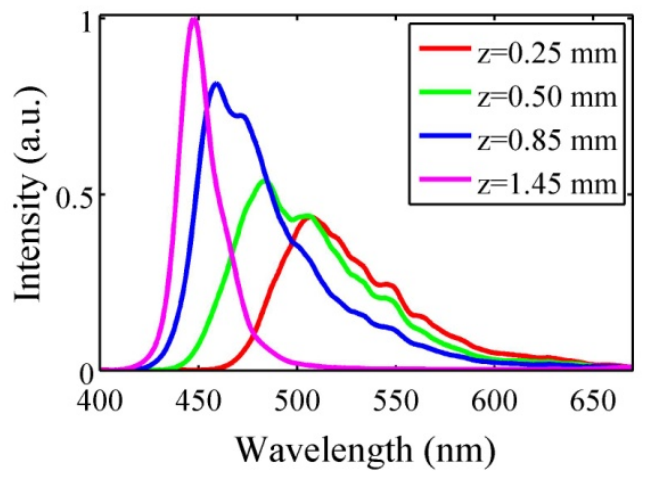

Figure 6.8. SC spectra extracted from the map in Figure 6.7 a) at the z positions indicated in the legend.

In addition to the tuning of the central wavelength of the peak, its width becomes smaller as the focal region moves towards the rear face of the crystal (increasing $z$ ). In particular, for the highest value of $z$, the spectral width of the peak is about $21 \mathrm{~nm}$, supporting about $14 \mathrm{fs}$ Fourier transform- 
limited pulses. In this case in particular, after removing the IR part of the spectra with the band-pass filter (FGB37s, Thorlabs) and taking into account its transmittance the energy of the pulses was approximately $4 \mathrm{~nJ}$.

Once the continuum had been generated, we allowed the beam to propagate and projected it on a screen (with no filter); we took several pictures with a CMOS-based digital camera (Canon, Powershot G10). As we proceeded in the recording of the spectral maps, for each different picture we changed the relative distance $(\mathrm{z})$ between the crystal and the lens. The set of measurements in Figure 6.9 was taken with a fundamental energy of 1.4 times the threshold $(E=1.38 \mu \mathrm{J})$ with no iris; i.e., in the same experimental conditions as before. The weak annular pattern that appears near the centre of each frame is unconverted IR light (above all in pictures corresponding to relative positions $\mathrm{z}=0 \mathrm{~mm}$ and $\mathrm{z}=-0.5 \mathrm{~mm}$ ). The spatial profile of the SC light is of fairly good quality. As expected the spectral features of this continuum can be envisaged in the snapshot.
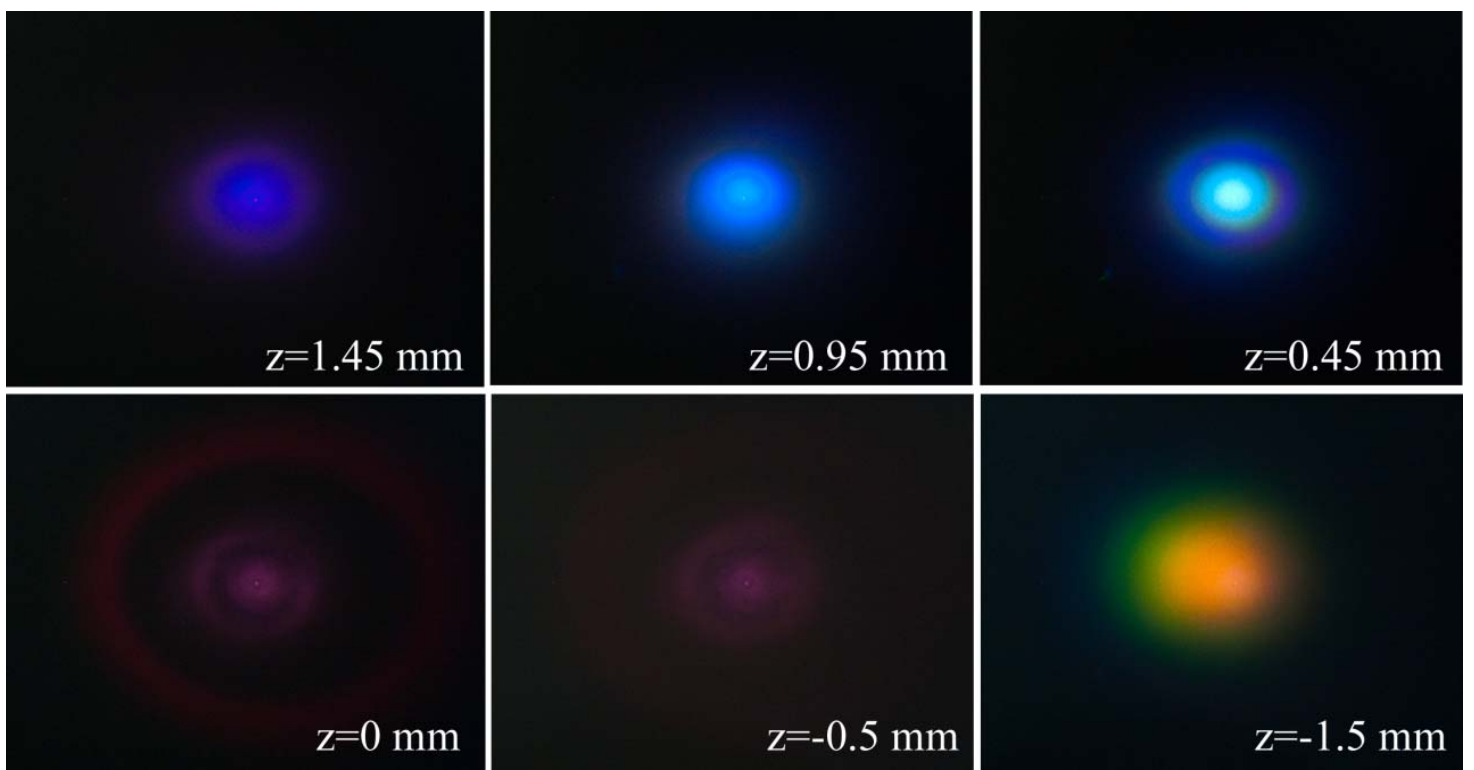

Figure 6.9. Pictures of the SC generated in a 3-mm thick sapphire plate after being projected onto a screen. $E=1.38 \mu \mathrm{J}$, no iris.

The movement of the sample along the propagation axis, $\mathrm{z}$, was larger than in the previous measurements. This is the reason for the negative $\mathrm{z}$ values indicated in the figure. The sequence was done by increasing the lens-sample relative distance (decreasing $\mathrm{z}$ ), starting from the position where the focus of the lens is at the rear part of the sapphire $(\mathrm{z} \cong 1.5 \mathrm{~mm})$. In the first three panels of this set of pictures we see how the SC is generated with dominant blue wavelengths. In this case, the spectral content should be similar to the one shown in Figure 6.8. Surprisingly in the next two panels there is no SC generation. We verified this fact with the spectrometer and only a modulation in the spectra due to self-phase modulation appeared. To conclude, in the last panel there is a continuum that is more similar to the one generated with a conventional focusing element. A typical associated spectrum could be the one presented in Figure 6.4. In our opinion, this situation appears when the sample is distant from the lens and the focal region is completely out of the same, and therefore the beam is divergent at the entrance of the sapphire plate.

In an ensuing set of measurements we raised the energy to $3 \mu \mathrm{J}$, and the iris was closed to a diameter of $4 \mathrm{~mm}$. This corresponds to an energy of 1.1-fold the threshold for these focusing 
conditions. The set of photographs associated with these measurements is shown in Figure 6.10. In this second set of measurements, the effect of the absence of the continuum disappeared, so it may be assumed that the effect was caused by the tighter focusing, although we do not have any feasible explanation for this. Nevertheless, the spectral content seems to change from one image to the other, proving that the effect of tunability persists. In Sect. 6.4.2, we shall see the spectra corresponding to this experimental situation.

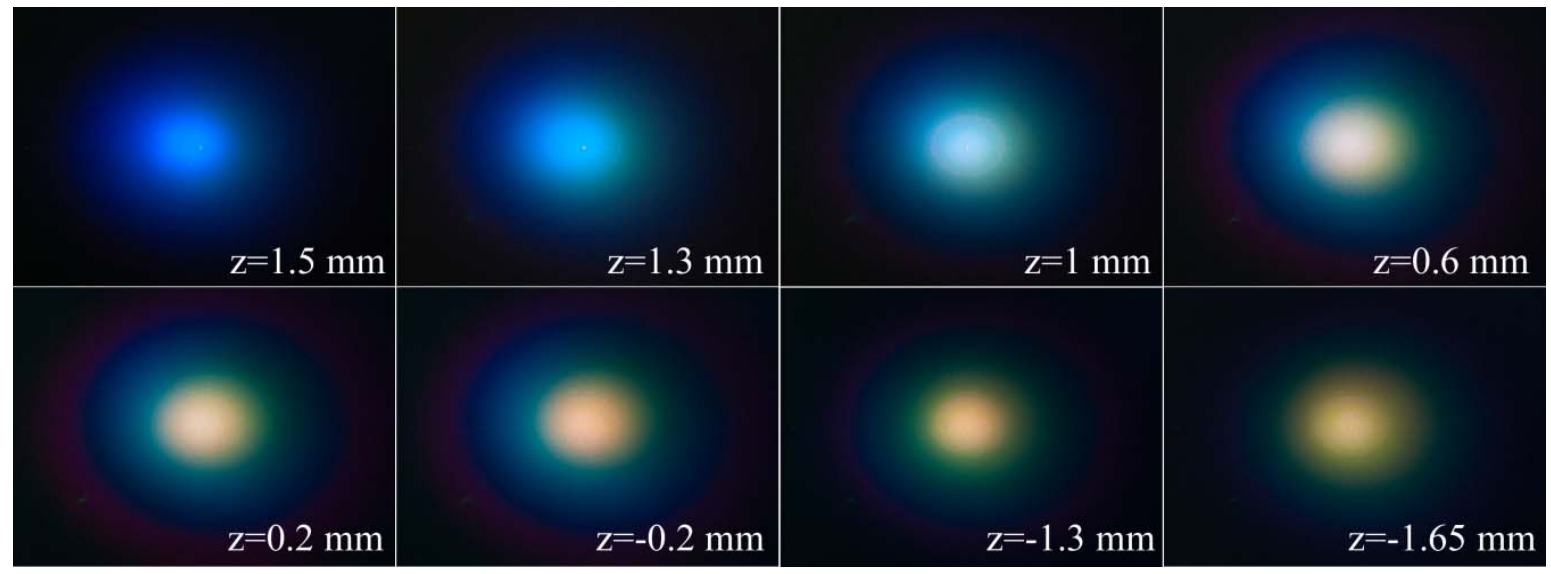

Figure 6.10. Images of the SC generated in a 3-mm sapphire plate after being projected onto a screen. $E=3 \mu \mathrm{J}$ and iris diameter $D=4 \mathrm{~mm}$.

\subsubsection{Energy and Wavelength Stability Measurements}

Below we present the measurements performed in order to confirm the validity of the SC pulses thus generated for use in other experiments; in particular, we tested the stability of both the wavelength and energy.

For this set of experiments we adjusted the energy of the incident pulse to $1.38 \mu \mathrm{J}$ and for a fixed position of the sample: the same as in the previous section the 3-mm thick one. We recorded a spectrum of SC every 5 seconds, with an integration time in the spectrometer of $40 \mathrm{~ms}$ over a time interval of $500 \mathrm{~s}$. We repeated this measurement for two different relative positions of the lens and the plate, $\mathrm{z}=1.4 \mathrm{~mm}$ and $\mathrm{z}=1.6 \mathrm{~mm}$.

Concerning wavelength stability, we calculated the average wavelength for each spectrum measured following the expression:

$$
<\lambda>_{t}=\frac{\sum_{\lambda} S(\lambda, t) \lambda S(\lambda, t)}{\sum_{\lambda} S(\lambda, t) S(\lambda, t)}
$$

where $S(\lambda, t)$ represents the spectral intensity at each time $t$. Then, we calculated the standard deviation, $\sigma_{<\lambda\rangle_{t}}$, as follows

$$
\sigma_{<\lambda\rangle_{t}}=\sqrt{\frac{\left.\sum_{i=1}^{N}(<\lambda\rangle_{t}-\overline{<\lambda\rangle_{t}}\right)^{2}}{N}}
$$


where $N$ is the total number of measurements. The results obtained are depicted in Figure 6.11. The blue line corresponds to $\mathrm{z}=1.4 \mathrm{~mm}$, and the green one to $\mathrm{z}=1.6 \mathrm{~mm}$. The horizontal lines are the arithmetic means (463.4 nm -blue- and $444.5 \mathrm{~nm}$-green-) and the standard deviation ( $0.97 \mathrm{~nm}$ and $3.02 \mathrm{~nm}$ respectively).

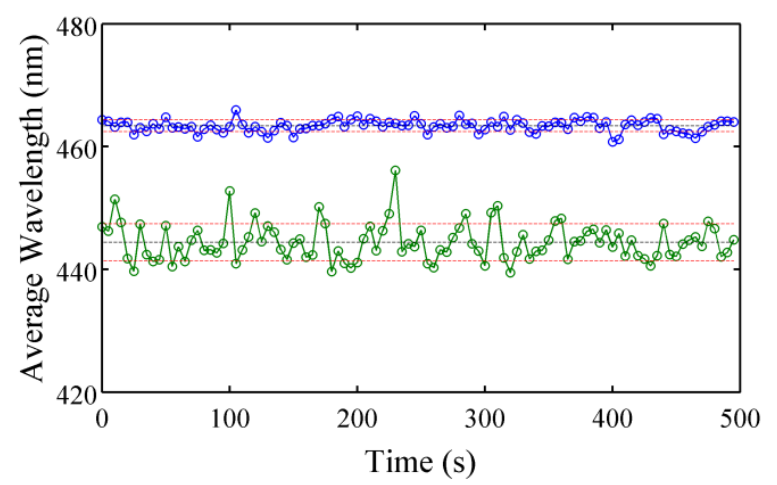

Figure 6.11. Stability of average wavelength of the SC generated in a 3-mm thick sapphire for two $z$ positions, $z=1.4 \mathrm{~mm}$-blue line - and $z=1.6 \mathrm{~mm}$-green line- .

We also wished to assess how energy-stable the pulses thus generated were, and hence we calculated the energy fluctuations using the same set of data. We considered the energy of each measurement, i.e., for a time $t$, as the integral over each spectrum, $S(\lambda, t)$, and then we calculated the mean of these energy values, $\bar{E}$. Finally, to gain a idea of the relative energy fluctuations we calculated an error for each value, $\delta E_{t}=\left|E_{t}-\bar{E}\right|$, and we represent $\frac{\delta E_{t}}{\bar{E}}(\%)$ in Figure 6.12. As before, the blue line represents the value $\mathrm{z}=1.4 \mathrm{~mm}$, and the green one $\mathrm{z}=1.6 \mathrm{~mm}$.

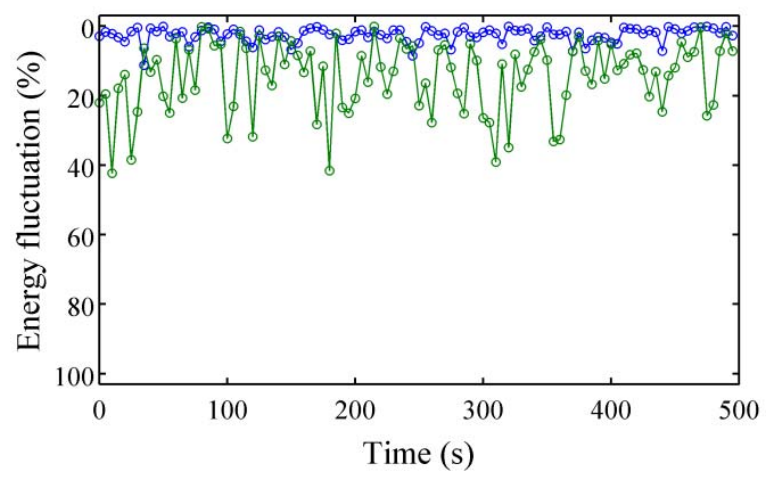

Figure 6.12. Representation of the energy fluctuations for the SC generated in a sapphire sample of 3-mm thickness and for two relative positions $z$, of the lens and the sample $z=1.4$ $\mathrm{mm}$-blue line- and $\mathrm{z}=1.6 \mathrm{~mm}$-green line-.

It is clear from the results that both the spectral and energy stability are quite good but they become worse as the peak is shifted to the blue border, (a larger value of $\mathrm{z}$ ). This can be understood since the focal region reaches the output face of the crystal, and it probably does not fit inside it completely. This is detrimental for the proper formation of the filament, thus reducing the possibility of SC generation, as argued in the previous section. However, when the lens-crystal 
distance is slightly higher (a lower value of $z$ ) stability is very good, with a total variation of less than $1 \mathrm{~nm}$ in wavelength and less than $1 \%$ in energy.

\subsubsection{Dependence of the SC on Parameters of the Input Beam: NA and Energy}

Regarding the role of the NA in the generation of the SC, we can refer to the literature, where it is generally believed that for the same energy conditions SC occurs for weakly focused beams (low NA) rather for strongly focused ones, where damage to the material is more likely to happen. However, there is also another characteristic in the continuum related to the NA. This is the extension of the blue edge, or blue-broadening [100]. The edge of the blue broadening is defined as the wavelength where the intensity has dropped to $10 \%$ of the average intensity of the flat pedestal. In this respect it was reported that when focusing with a conventional refractive lens, the blue broadening decreases upon increasing the NA.

For the next experiment we decreased the numerical aperture by limiting the input beam size, interposing an iris with a diameter of $5.5 \mathrm{~mm}$ in front of the DL (see Figure $6.5 \mathrm{~b}$ ), corresponding to a NA=0.026. However, we observed that upon decreasing NA (the previous value of NA was 0.04), the blue broadening also decreased. This behaviour can be observed in the spectral map depicted in Figure 6.13 a). This map was taken in the same way as in Figure 6.7 a), moving the sample towards the lens. The horizontal axes were set the same in the two figures to clarify that the extension of the blue edge has decreased. Moreover, the spectral content is different in the sense that there is some wavelength generation in the red part of the spectrum. This can be seen more clearly in the Figure $6.13 \mathrm{~b}$ ), where we present cuts from the map in a). Note, however, that the spectra were extracted from the right side of the figure, corresponding to situations in which the sample was closer to the lens. This figure must be compared with Figure 6.8; in that case, the spectra showed a negligible content in red wavelengths while in this case (Figure $6.13 \mathrm{~b}$ ) they did not. However, the peak was moved to the red as $\mathrm{z}$ was increased, although tunability was still present.

If we take a look back at the shots of Figure 6.10 we see the difference in colour with respect to the other case with no iris. Moreover, the shots are consistent with the spectra shown in Figure 6.13.
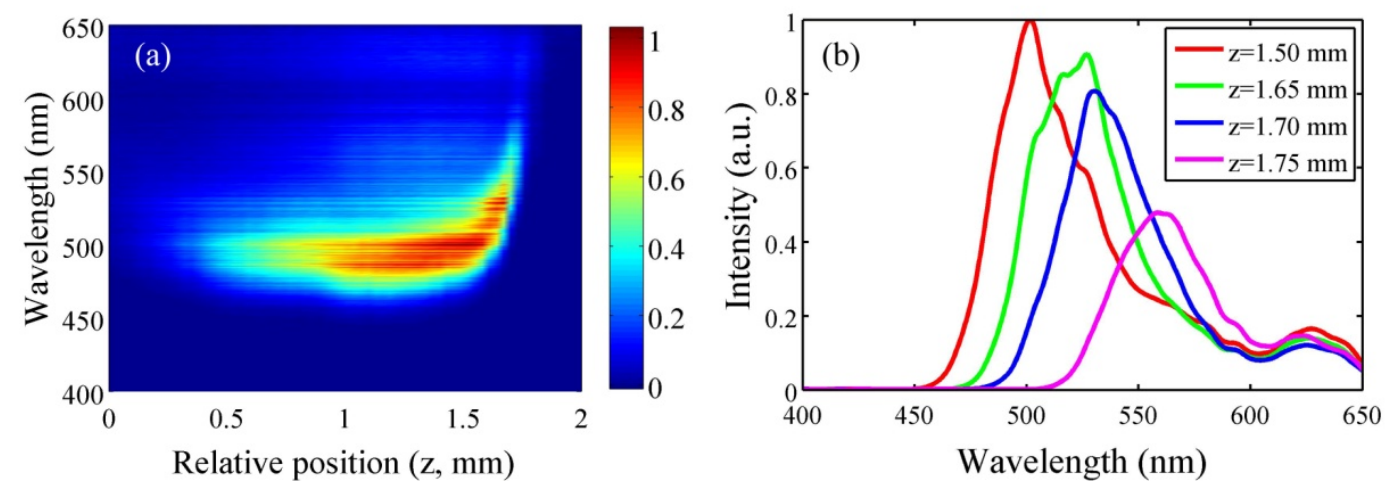

Figure 6.13. a) Spectra of SC generated in sapphire with the DL and an iris diameter of 5.5 $m m, N A=0.026$. The pulse energy was set to $0.98 \mu \mathrm{J}$. The colour scale represents normalized intensity in arbitrary linear units. b) Different spectra taken from the map on the left in the $z$ positions indicated in the legend.

The second part of this section was devoted to studying how the input energy affects the SC generated with a DL. To do so, we fixed a $\mathrm{z}$ position, specifically $\mathrm{z}=1.4 \mathrm{~mm}$, we removed the iris, 
and the rest of the experimental conditions were the same as in previous experiments. Then, we recorded the spectra while changing the input energy. The results are shown in the figure below.

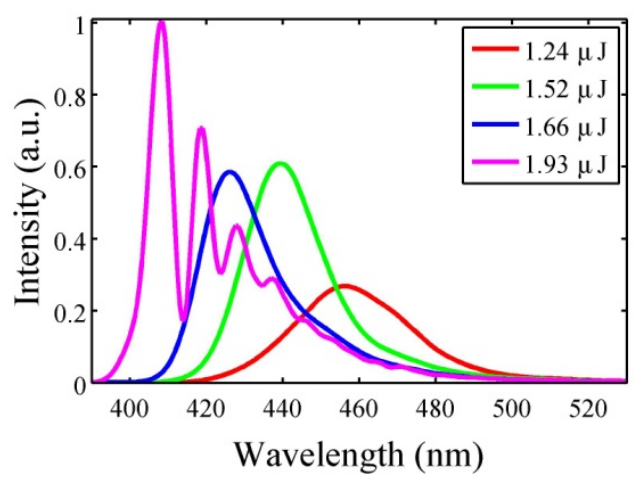

Figure 6.14. Study of the energy dependence of the SC generated in a sapphire with a DL.

We observed that as the energy was increased, the central wavelength of the peak moved to shorter wavelengths. In the literature [102] there are examples of how the energy of the input pulse affects the spectral content, but not as clear as seen in our case, so this new phenomena can be related to the focusing with the DL. Note how when the energy surpasses a value of 2 times the threshold, input energy $1.93 \mu \mathrm{J}$, the border drops to $400 \mathrm{~nm}$. However, the spectrum becomes strongly modulated and stability is poor. This can be attributed to the appearance of multifilamentation [106].

\subsubsection{Testing Another Sample Thickness and Material}

At this point, having studied the 3-mm thick sapphire plate in detail we considered that it was also important to study the effects, or the dependence, on sample thickness, on the one hand, and test the behaviour of other materials, on the other.

a)

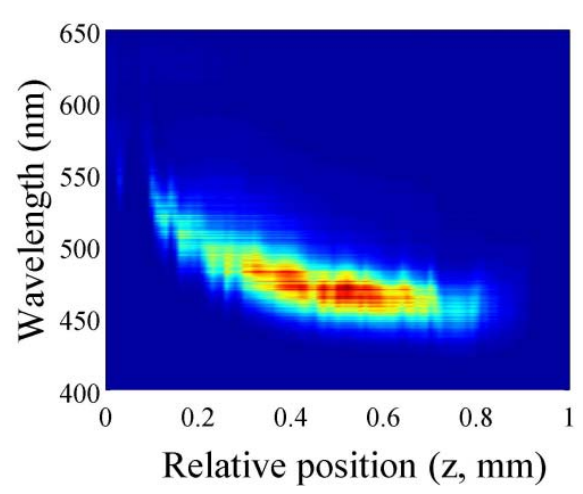

b)

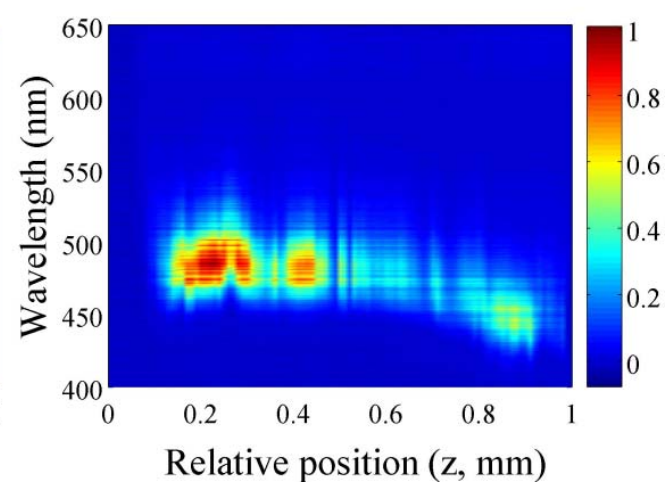

Figure 6.15. Spectral maps taken in sapphire samples of a) 2-mm and b) 1-mm thickness. The colour scale represents normalized intensity in arbitrary linear units.

We chose two sapphire plates of 1- and 2-mm thickness that were available at the laboratory, and we recorded the spectra of the SC generated with the DL. In this case, with no iris, we used an incident pulse energy of 1.4-fold the threshold, in agreement with the measurements in Table 6.1. The data were plotted in the same way as before and we preserved the same wavelength axis to 
make them easier to compare with previous maps. The results are depicted in Figure 6.15. a) and b) for the 2-mm and 1-mm samples respectively.

In both cases the tunability with $\mathrm{z}$ is present in a similar way as with the $3-\mathrm{mm}$ thick sample. The spectra have a negligible content in red wavelength, something that also happens with the thicker sample, with no iris. However, when the 1-mm crystal was used, the shape of the map was different and the stability of the SC was considerably worse than in the other two cases. As previously discussed, the 1-mm thickness is smaller than the focal region of the DL lens, which is clearly detrimental for the proper formation and propagation of the filament.

To complete the study we performed some measurements in fused silica. The most relevant was the one corresponding to a 3-mm thick fused silica sample. In this case, the input energy was 1.4fold the threshold for this material and these experimental conditions, which involved focusing with a DL and without an iris. A representative map of wavelength-distance (z) can be seen in Figure 6.16 .

The structure is similar to that previously reported: a negligible spectral content in the red and a prominent peak in the blue border of the SC. As mentioned before, SC generation in fused silica is less stable than the SC in sapphire. This is shown in the picture lacking spectra at some positions of $\mathrm{z}$, and also with a reduction in the intensity. The smallest stability of the SC generated in fused silica in comparison to that of sapphire can be associated with the long-term degradation of the material. In sapphire, the absence of degradation can be attributed to a high thermal conductivity and low absorption in the UV [89]. Additionally, the same kind of tunability is found when varying $\mathrm{z}$ : the central wavelength of the peak shifts towards the blue as $\mathrm{z}$ is increased. However, the region from which it is possible to obtain a stable continuum extends over $\sim 0.4 \mathrm{~mm}$, a value much lower than the $1.5 \mathrm{~mm}$ attained with the sapphire crystal with the same thickness; see Figure 6.7 a).

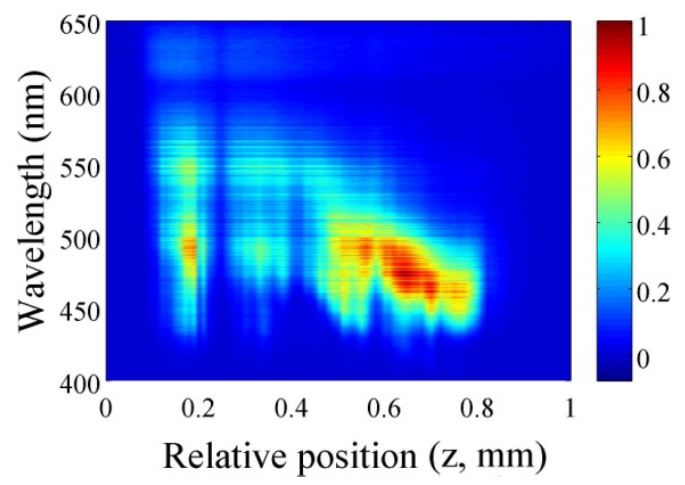

Figure 6.16. Spectral map taken for a 3-mm fused silica sample with the DL and an input energy of 1.4 the threshold for SC generation $(1.74 \mu \mathrm{J})$. The colour scale represents normalized intensity in arbitrary linear units.

\subsection{Conclusions}

In conclusion, we have experimentally studied the spectral behaviour of visible SC generated by focusing near IR femtosecond pulses with a kinoform DL into sapphire. To avoid damage to the crystal and multi-filamentation, in all the experiments the energy of the pump pulses was set between 1 and 2 times the measured generation threshold. The spectra of the continuum thus generated exhibit a strongly peaked structure at the blue border, with a residual content of red 
wavelengths. For a given pulse energy, the central wavelength of this peak can be accurately shifted from over a range greater than $50 \mathrm{~nm}$ by simply changing the relative distance between the DL and crystal. In our opinion, these are two observations that differ from the behaviour of the SC when focusing with refractive lenses and that may be highly suitable for some applications. Similar results were found in different sample thicknesses and in another commonly used material; fused silica.

The observable effects can be attributed to the long focal region of the DL, due to the strong chromatic aberrations characteristic of this lens. Moreover, on comparing these results with those obtained using an achromatic refractive lens, under analogous conditions (NA and pulse energy), we found that the maximum extension (blue border) of the spectral power was significantly larger with the DL.

According to our results, the use of a DL for SC generation in bulk exhibits very interesting features for the spectral control of the light generated. We also tested the stability both in wavelength and energy of the SC pulses generated, obtaining very good results. This suggests that this could be a useful source applicable in other experiments.

We believe that the SC pulses obtained this way may have wide applicability, e.g. for seeding ultrafast optical parametric amplifiers or for spectroscopic measurements. Therefore, a complete spatial and temporal characterization of these pulses is necessary. This work is now in progress [107]. 



\section{Chapter 7}

\section{Design and Implementation of a Noncollinear OPA in the Visible for fs Pulses}

\subsection{Introduction}

In this chapter we shall describe the design and implementation of a Noncollinear Optical Parametric Amplifier (NOPA) in the visible, which has been the first tunable source at the laboratory in Salamanca. The field of OPAs, from low energy OPOs to OPCPA systems, has evolved rapidly and is of great relevance in many scientific disciplines. Here we shall focus only on femtosecond OPAs, but even within this division the designs may be very different, although they do share some key elements. In this part, we shall address some basic principles underlying the different implementations.

In particular, femtosecond visible OPAs are important for a variety of spectroscopic applications, because many systems of interest in physics, chemistry and biology have absorption bands in this wavelengths range. Therefore, it is very interesting to have a tunable source in this region, and this is indeed the main motivation of having available a visible OPA developed at the laboratory in Salamanca. Our aim was to expand the number of secondary sources available in the laboratory so that they could be applied to materials micro-processing, while at the same time gaining experience in a technology for which there was no previous background at the Universidad de Salamanca. 
The chapter is organized as follows. First, we shall discuss the state of the art in the OPA field. Then, we shall explain two important issues in the design of an OPA: choice of the pump and signal beams. Following this, some theoretical considerations will be given in order to support some effects seen with femtosecond OPAs. In particular, the basis of broadband parametric amplification will be described along with an experimental example of the OPG process. We shall then describe the NOPA developed and installed in Salamanca. The first amplification stage of this system is used in the characterization of SC pulses generated in fused silica and sapphire plates. Next, we shall describe a NOPA system that was installed in Bordeaux during part of the thesis period; the system had some characteristics in common with the one in Salamanca, but also some improvements, which in our opinion deserve mention. To conclude the chapter we shall comment on our future intentions in relation to possible new OPAs to be developed in Salamanca. A discussion about the two amplification schemes, OPCPA and conventional CPA, applied to the generation of high peak power laser systems is included at the end of this chapter.

\subsection{Femtosecond OPAs}

With the processes described until now, second- and third-harmonic generation, we have seen that it is possible to expand the range of emission of Ti:Sapphire laser systems. However, as was shown with these processes we can only generate fixed-frequency bands. We could have gone further and generated even the fourth harmonic [80] and gone down in wavelengths to approximately $200 \mathrm{~nm}$, but we would not have been able to go much further than that, because we would have found problems of absorption in the nonlinear crystals. In any case, with such processes it is not possible to generate frequencies in the middle: for example, between the second harmonic and the fundamental. Here is where OPAs appear to solve the problem. In contrast, the processes of OPA, OPG and OPOs allow us to expand the spectral range continuously, as we shall see later in the chapter. Moreover, in the case of our experimental arrangement they allow us to obtain a tunable source in almost all the visible range, and in other approaches the same tunability is obtained in the infrared region. Although we shall focus our study on the visible spectrum, some references to the IR case will be made at some point, because while they have some similarities, they are also different.

Femtosecond OPAs have become the most widespread source of tunable femtosecond pulses and have reached a high-level of stability and reliability, becoming true workhorses in disciplines such as ultrafast spectroscopy [66]. These OPAs have demonstrated tunability from the UV [108] to the mid-IR, in the range of 5 to $9 \mu \mathrm{m}$ [109]. In addition, they have the ability to generate pulses significantly shorter than the pump pulses, exploiting the broad gain bandwidths available in the parametric interaction. In fact ultra-broadband pulses with durations down to approximately $5 \mathrm{fs}$ in the visible [110] and 15 fs [111] in the IR have been obtained, containing only a few optical cycles of the carrier frequency. The record to date is $4 \mathrm{fs}$ in the visible-near-IR region [112], obtained from a noncollinear blue-pumped system with the help of an adaptive pulse compressor. Moreover, the OPA process offers the possibility of generating pulses with the carrier-envelope phase locked [113] in a passive all-optical stabilization process, which is a very interesting feature when working with few-cycle pulses.

These systems based on OPA are, however, limited in terms of generating high-power few-cycle pulses, and other approaches have been demonstrated to solve this problem, based on the coherent superposition of a multiband octave spanning spectrum obtained by nondegenerated four-wave 
mixing $[114,115]$, or on the temporal compression of the broadband SC generated in gas filled hollow fibres [116]. Also self-compression by filamentation in gases holds promises to be a mean of generate these high-power pulses with carrier envelope phase stabilization [117, 118].

In comparison with OPOs, OPA systems have two drawbacks: on the one hand they require high pump intensities, provided only by an amplified system, and on the other hand they operate at low repetition rates, typically from 1 to $100 \mathrm{kHz}$, while OPOs can operate in the $\mathrm{MHz}$ regime. However, in our particular case, we cannot consider these issues as problems because we have an ultraintense source available, with an adequate repetition rate for OPA operation. Moreover, OPAs have some advantages. For instance, they provide high output energies in one pass (travelling wave); they offer broadband frequency tunability in noncollinear geometry and they are much simpler to operate than OPOs since they do not require any cavity-length stabilization.

Regarding OPAs, several schemes exist. In our opinion an excellent review made by G. Cerrullo and S. De Silvestri is a good paper to gain a general idea of this active field of research [89]. This article summarizes the progress made in the development of ultrafast OPAs, giving the basic design principles for the generation of radiation in each of the different frequency ranges where the OPA was demonstrated. We start here by revising some of the key points concerning their design.

\subsection{Design of an OPA in the Visible}

There are three key elements in an OPA: the pump beam, the seed (i.e. the signal to be amplified) and the nonlinear crystal where the interaction takes place. Other complementary elements may include a chirp compensation scheme, beam modulators, etc. Another important issue is the type of interaction, i.e. collinear or noncollinear, as well as the type of phase matching (Type I or Type II). The combination of all these elements is what determines the features of the amplified signal at the output of the system. In this section we shall focus on two of the three key elements: the signal and the pump beams.

In the case of femtosecond OPAs, there are two main pump beams: the fundamental $(\sim 800 \mathrm{~nm}$, central wavelength) and second-harmonic pulses $(\sim 400 \mathrm{~nm})$ from regeneratively amplified modelocked Ti:Sapphire laser systems. As we shall see with the fundamental pumping, usually called IR pumping, we can generate new frequencies in the IR, and with the visible/blue pumping we can generate frequencies in the visible region. In the case of more energetic OPCPA systems, the pumping is the second harmonic of Nd:YAG lasers (532 nm) [119].

It should be recalled that the nonlinear process underlying an OPA is the DFG process. Note that we have introduced this process in Chapter 2: two monochromatic waves with frequencies $\omega_{1}$ and $\omega_{3}$ were incident in the nonlinear medium and the $\omega_{2}=\omega_{3}-\omega_{1}$ frequency was generated. Additionally, when the wave at $\omega_{3}$ was intense, the amplification of $\omega_{1}$ occurred as a result of the interaction. Heceforth the wave at frequency, $\omega_{3}$, will be called the pump wave, $\omega_{p} ; \omega_{1}$ will be the signal or seed wave, $\omega_{s}$; and $\omega_{2}$ will be the idler, $\omega_{i}$. Therefore, the energy conservation law simply transforms to:

$$
\omega_{p}=\omega_{s}+\omega_{i}
$$

Similarly, in terms of wavelengths we have: 


$$
\frac{1}{\lambda_{p}}=\frac{1}{\lambda_{s}}+\frac{1}{\lambda_{i}}
$$

The plots in Figure 7.1, represent the energy conservation law, and show us the idler wave that is generated with each corresponding signal. In order to calculate them, we have fixed the pump wavelength and have allowed the signal and idler wavelengths to vary as follows from Eq. (7.2).

a)

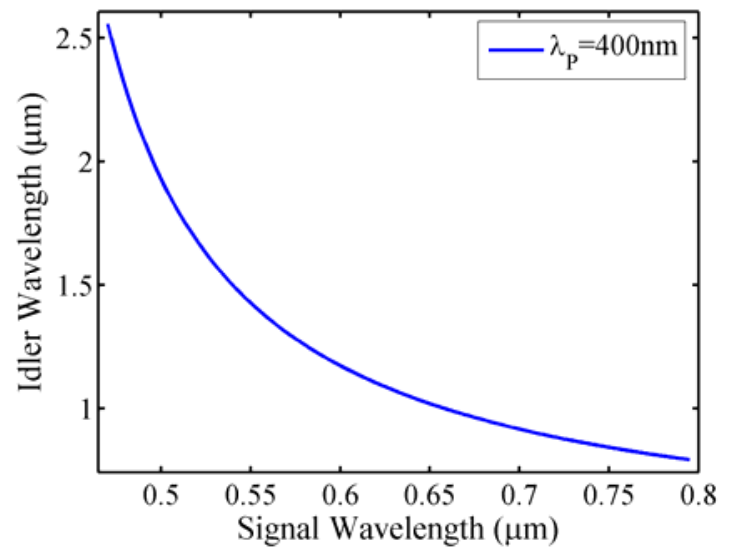

b)

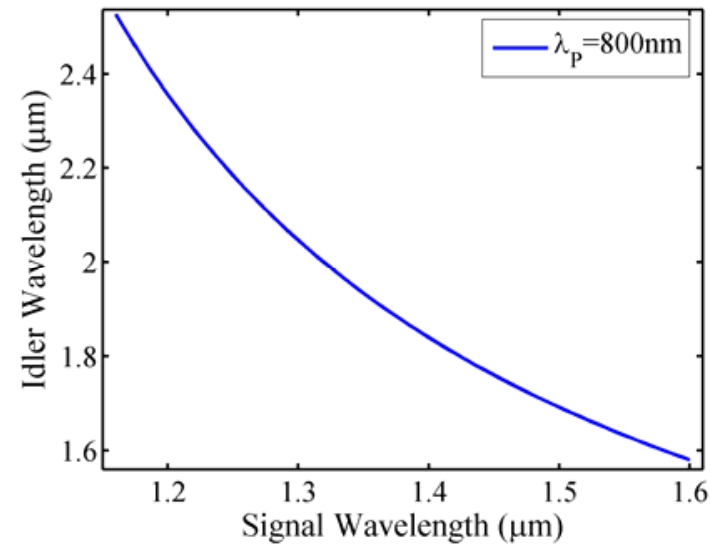

Figure 7.1. Plots of the energy conservation law under the OPA process, Eq. (7.2), which links each signal wavelength with the corresponding idler, when the OPA is pumped by a) the second harmonic and b) the fundamental of the Ti:Sapphire laser.

In principle, the signal frequency to be amplified can vary from $\omega_{\mathrm{p}} / 2$, which is the so-called degeneracy condition (when $\omega_{s}=\omega_{i}$ ), to $\omega_{\mathrm{p}}$. Correspondingly, the idler can vary from $\omega_{\mathrm{p}} / 2$ to zero (when $\omega_{s}=\omega_{p}$ ). Therefore, in terms of wavelengths, the signal could vary from $800 \mathrm{~nm}$ to $400 \mathrm{~nm}$ in the case of blue pumping, and from $1.6 \mu \mathrm{m}$ to $800 \mathrm{~nm}$ in case of the IR pumping. However, in practice not all the wavelengths that satisfy the energy conservation law can be generated. The absorption of the infrared wavelengths in the crystal is responsible for this behaviour; i.e., when the signal wavelength is close to that of the pump the corresponding idler falls in the IR beyond 2.5 $\mu \mathrm{m}$, which is the limit of IR absorption of the BBO nonlinear crystal ${ }^{14}$. Therefore, these signals are not shown in the plots of Figure 7.1. The consequence of idler absorption is more evident in the case of IR pumping, because signals lower than the $1.2 \mu \mathrm{m}$ wavelength cannot be amplified, leaving a larger gap with the pump at $800 \mathrm{~nm}$ than in the blue pumping case, where the lowest signal wavelength that can be amplified is $\sim 450 \mathrm{~nm}$.

Since the infrared limit in the case of KDP is much lower than in the case of the BBO, the latter is preferable to KDP in some OPA designs. However KDP is used in high peak-power OPCPA schemes because it can be grown to huge sizes [4]. For OPAs in the IR region other crystals with a higher transparency range must be used: for example, $\mathrm{LiIO}_{3}$ [120], $\mathrm{AgGaS}_{2}$ or $\mathrm{HgGaS}_{4}$ [109].

In our case, since we were interested in generating frequencies in the visible spectrum, we needed to chose the second harmonic of the Ti:Sapphire laser system as the pump. As in the previous chapters $\mathrm{BBO}$ was be the crystal chosen.

\footnotetext{
${ }^{14}$ We extracted this value from the transmission curve (Figure 3.6) plotted in the Chapter 3, and considered this limit when the transmission decreases to $50 \%$.
} 


\subsubsection{Generation of an Appropriate Signal}

In the previous section we have been talking about the possibilities in the choice of the pump beam. In this section we shall address the generation of an appropriate signal. As in the case of pumping, several possibilities appear; what makes one approach preferable to others has to do with the energy, the bandwidth extension, and the wavelength content.

The signal beam must be a broadband pulse, which is usually obtained by making use of the processes described in the previous chapter; i.e., processes involving the nonlinear propagation of femtosecond pulses, such as self-focusing and filamentation, which result in the generation of new frequencies. There are different ways to obtain the signal beam: for example, filamentation in a gasfilled hollow fiber [111, 121], or SC generation either in photonic-crystal fibres [122] or in solids such as sapphire [123], fused silica [124] or calcium fluoride [125].

The idea is to have a very broad spectrum covering the whole visible range in the case of the blue-pumped OPA, or a spectrum that spans the infrared in the case of IR-OPAs, because, as expected, the signal must have a wavelength content that matches the range in which we wish to generate the amplification. For example in the case of SC generation in sapphire, in order to have a signal in the IR it is preferable to use a $3-\mathrm{mm}$ thick plate, while for generating frequencies in the visible region a 1-mm thick sample is more adequate. Apart from the fact that it is easier to generate the IR frequencies when the sample is thicker ${ }^{15}$, the dispersion introduced is reduced if, for the visible $\mathrm{SC}$, we use a sample as thin as possible.

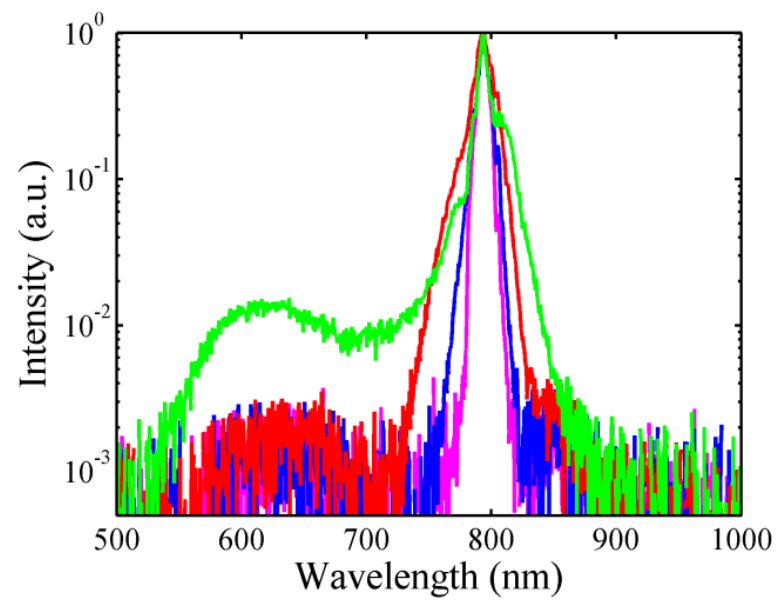

Figure 7.2. Spectra of the SC pulses generated in a fused silica plate of 6-mm thickness. The different curves correspond to different relative positions between the focusing lens and the plate. The pink line corresponds to the spectrum of the laser.

In the case of the best SC signal adapted to our experimental conditions, we decided to start working with fused silica plates, testing samples of different thickness, and changing the focusing conditions, (i.e lens focal length and iris diameter) in order to obtain the configuration that would provide the best performance in terms of stability, wavelength extension, and spatial shape. Figure 7.2 shows a typical SC spectrum - green line- generated in fused silica. In this plot we have represented the spectra recorded in different relative positions between the plate and the focusing

\footnotetext{
${ }^{15} \mathrm{We}$ do not have a reference for this, but Dr. D. Brida provided us with this information after a discussion addressing the issue.
} 
lens $(\mathrm{f}=30 \mathrm{~cm})$, in a similar way to what was done in the previous chapter. The pink line corresponds to the spectrum of the laser, and in the blue and red lines the symmetric broadening characteristic of self-phase modulation can be seen clearly; in such cases the SC had not set in.

Following the work done by other researchers we replaced fused silica by sapphire [123]. From a practical point of view, and based on our own experimental observations, we can state that sapphire is more resistant to degradation than fused silica. The SC in sapphire remains stable along more time of operation. As mentioned in the previous chapter, this can be attributed to its high thermal conductivity and low absorption in the UV [89].

We carried out the same kind of measurement as in the fused silica case and the results are displayed in Figure 7.3 (for a 3-mm thick sample). Again the spectrum of the incident pulse is included for comparison. We see how the blue edge in the case of the sapphire spans lower wavelengths in comparison with the fused silica. As mentioned in the previous chapter the higher $E_{\text {gap }}$ of the sapphire is responsible for the greater extension in the blue part of the spectrum [101].

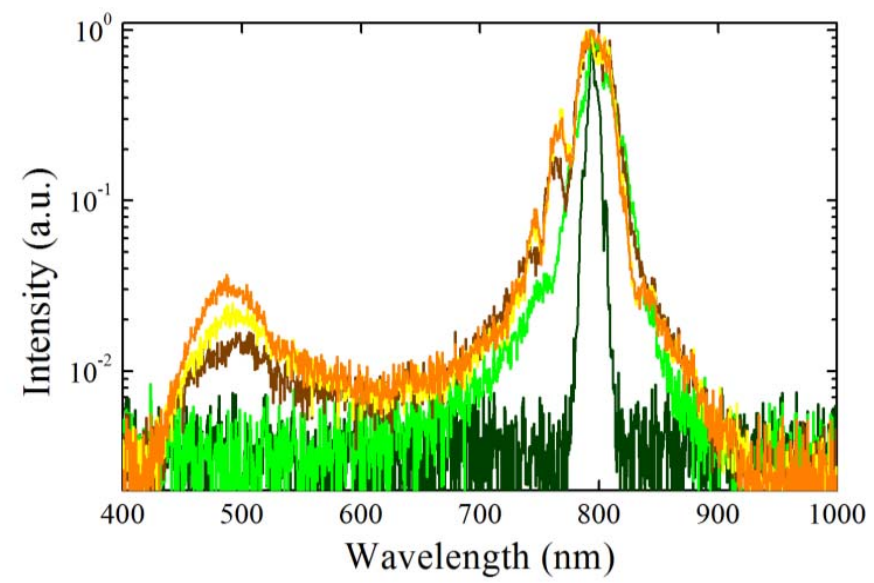

Figure 7.3. Spectra of SC pulses generated in a 3-mm thick sapphire plate. The different curves correspond to different relative positions between the focusing lens and the plate. The dark green line corresponds to the spectrum of the laser.

However, the SC generated in bulk also has some drawbacks. For example, an intense peak in the fundamental wavelength is always present and hence the OPA nonlinear crystal may be damaged, even when using low energy, because sometimes the focal spot lies inside the crystal. Another drawback is the introduction of temporal chirp due to dispersion in the material. As mentioned there are other possibilities in the generation of the seed, as is the case of the SC generated in photonic crystal fibres, where the chirp of SC pulses can be controlled simply by changing the fibre size. Nevertheless the output energy is lower [122].

\subsection{Optical Parametric Amplification with Femtosecond Pulses: Theoretical Model}

Similarly to what we saw in the case of the second harmonic, in Chapter 2 we described the phenomenon of parametric amplification when monochromatic plane waves were involved. However, since we shall be working with femtosecond pulses new considerations must be made, again, having to do with the effects of materials dispersion. 
In the slowly varying amplitude approximation, and neglecting pulse lengthening due to second or higher-order dispersion effects (which as was also discussed in Chapter 3 is a good approximation when working with $100 \mathrm{fs}$ pulses and not very thick crystals: $\sim 1 \mathrm{~mm}$, as is our case), we obtain the following system of coupled wave equations which describe the OPA process [126]:

$$
\begin{aligned}
& \frac{\partial A_{s}}{\partial z}+\frac{1}{v_{g s}} \frac{\partial A_{s}}{\partial t}=-j \frac{\omega_{s} d_{e f f}}{n_{s} c} A_{i}^{*} A_{p} \exp (-j \Delta k z) \\
& \frac{\partial A_{i}}{\partial z}+\frac{1}{v_{g i}} \frac{\partial A_{i}}{\partial t}=-j \frac{\omega_{i} d_{e f f}}{n_{i} c} A_{s}^{*} A_{p} \exp (-j \Delta k z) \\
& \frac{\partial A_{p}}{\partial z}+\frac{1}{v_{g p}} \frac{\partial A_{p}}{\partial t}=-j \frac{\omega_{p} d_{e f f}}{n_{p} c} A_{s} A_{i} \exp (j \Delta k z)
\end{aligned}
$$

where

$$
\Delta k=k_{p}-k_{s}-k_{i}
$$

is the phase-matching condition and $A_{\mathrm{p}}, A_{\mathrm{s}}$ and $A_{\mathrm{i}}$ are the pulse envelopes of the pump, signal and idler respectively. Note that equations (7.3) also neglect third-order nonlinear effects (self- and cross-phase modulation).

Then, by transforming to a frame of reference that moves with the group velocity of the pump pulse $\tau=\left(t-z / v_{g p}\right)$, we obtain the equations:

$$
\begin{aligned}
& \frac{\partial A_{s}}{\partial z}+\left(\frac{1}{v_{g s}}-\frac{1}{v_{g p}}\right) \frac{\partial A_{s}}{\partial \tau}=-j \frac{\omega_{s} d_{e f f}}{n_{s} c} A_{i}^{*} A_{p} \exp (-j \Delta k z) \\
& \frac{\partial A_{i}}{\partial z}+\left(\frac{1}{v_{g i}}-\frac{1}{v_{g p}}\right) \frac{\partial A_{i}}{\partial \tau}=-j \frac{\omega_{i} d_{e f f}}{n_{i} c} A_{s}^{*} A_{p} \exp (-j \Delta k z) \\
& \frac{\partial A_{p}}{\partial z}=-j \frac{\omega_{p} d_{e f f}}{n_{p} c} A_{s} A_{i} \exp (j \Delta k z)
\end{aligned}
$$

Despite the many simplifications made to obtain the system of Eqs. (7.5), these equations do capture the main issues of parametric amplification with ultrashort pulses, which are related to the GVM between the interacting pulses, as happened in the case of the processes of second- and thirdharmonic generation described in previous chapters. In particular, the GVM between the pump and the amplified and generated beams (signal and idler, respectively) limits the interaction length over which parametric amplification takes place, while the GVM between the signal and idler beams limits the phase-matching bandwidth, as we are about to see.

We shall start by considering a collinear configuration. For this situation let us assume that perfect phase matching, $\Delta k=0$, is achieved for a given signal frequency $\omega_{\mathrm{s}}$ and for the corresponding idler frequency, $\omega_{\mathrm{i}}\left(\omega_{\mathrm{i}}=\omega_{\mathrm{p}}-\omega_{\mathrm{s}}\right)$. If the signal increases to a certain value, $\Delta \omega$, to $\omega_{\mathrm{s}}+\Delta \omega$, by energy conservation (7.1), the idler frequency decreases to $\omega_{\mathrm{i}}-\Delta \omega$. We can thus make a Taylor expansion to first order in the wave-vector mismatch: 


$$
\Delta k=\Delta k_{0}-\frac{\partial k_{s}}{\partial \omega_{s}} \Delta \omega-\frac{\partial k_{i}}{\partial \omega_{s}} \Delta \omega
$$

Since $\partial \omega_{s}=-\partial \omega_{i}$, as we have discussed, and $\Delta k_{0}=0$, we obtain the following expression:

$$
\Delta k=-\frac{\partial k_{s}}{\partial \omega_{s}} \Delta \omega+\frac{\partial k_{i}}{\partial \omega_{i}} \Delta \omega=\left(\frac{1}{v_{g i}}-\frac{1}{v_{g s}}\right) \Delta \omega
$$

We can also calculate the gain bandwidth, defined at the points for which the gain, $G(\lambda)$, decreases to $50 \%$ of the maximum value, $G_{0}$ (given by Eq. $(2.82)$ ), within the large-gain approximation, $\Gamma L \gg 1$, the gain bandwidth results $[89,127]$ :

$$
\Delta \lambda \cong \frac{2(\ln 2)^{1 / 2} \lambda^{2}}{c \pi}\left(\frac{\Gamma}{L}\right)^{1 / 2} \frac{1}{\left|\frac{1}{v_{g s}}-\frac{1}{v_{g i}}\right|}
$$

As can be deduced from Eq. (7.8), the large GVM between the signal and idler waves dramatically decreases the gain bandwidth. However, a high value of this quantity is expected when the OPA approaches the degeneracy point, $\omega_{s} \rightarrow \omega_{i}$, in Type I interactions. Recall that in such a case the signal and the idler are both ordinary waves [122, 128]. Evidently, in this case Eq. (7.8) loses validity and the phase-mismatch factor, $\Delta k$, must be expanded to the second order.
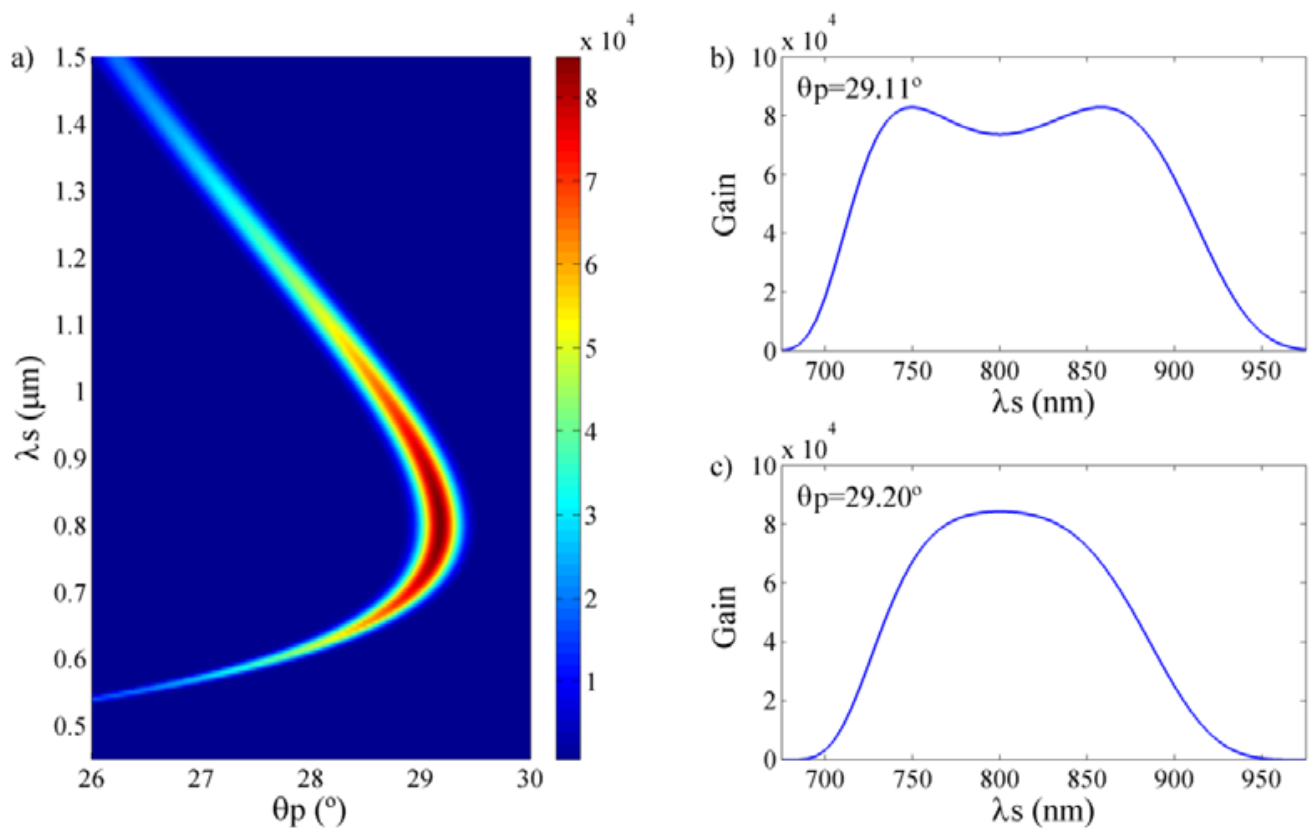

Figure 7.4. a) Gain in a collinear blue-pumped OPA $\left(I p=100 \mathrm{GW} / \mathrm{cm}^{2}\right)$ in $B B O$ as a function of signal wavelength and phase-matching angle. The colourbar represents gain factor in arbitrary linear units. b) and c) represent the gain bandwidth for two different phase-matching angles indicated in the figure. 
In Figure 7.4 we have plotted the gain, $I_{\mathrm{s}}(L) / I_{\mathrm{s}}(0)$, calculated as follows from Eq. (2.79) in the case of a Type I collinear OPA in BBO, pumped by the second harmonic of the Ti:Sapphire laser $\left(\lambda_{\mathrm{p}}=400 \mathrm{~nm}\right)$, as a function of the signal wavelength and the pump angle with respect to the optical axis of the crystal (the phase-matching angle, $\theta_{\mathrm{p}}$ ) at a pump intensity of $\mathrm{I}_{\mathrm{p}}=100 \mathrm{GW} / \mathrm{cm}^{2}$.

Note, on one hand, the high amplification factor that can be obtained, $\sim 10^{4}$, and on the other, the increase in gain bandwidth at the degeneracy point, $\theta_{\mathrm{p}}=29.2^{\circ}$ (Figure 7.4 panel $\mathrm{c}$ )), as we have anticipated from Eq. (7.8). We can see by comparing panels b) and c) that, with a slightly different $\theta_{\mathrm{p}}$ angle, the bandwidth increases at the expense of a modulation in the amplified spectrum.

\subsubsection{Noncollinear Phase Matching: Broadband Amplification}

So far we have only considered a collinear interaction in which once the phase-matching condition $(\Delta k=0)$ has been achieved, the group velocities of the signal and idler, and hence the phasematching bandwidth, are set. In this section we shall see that there is an additional degree of freedom in the noncollinear interaction: the pump-signal angle, $\alpha$ (see Figure 7.5). By suitably selecting this angle, it is often possible to achieve phase and group-velocity matching between signal and idler simultaneously, thus obtaining very broad gain bandwidths.

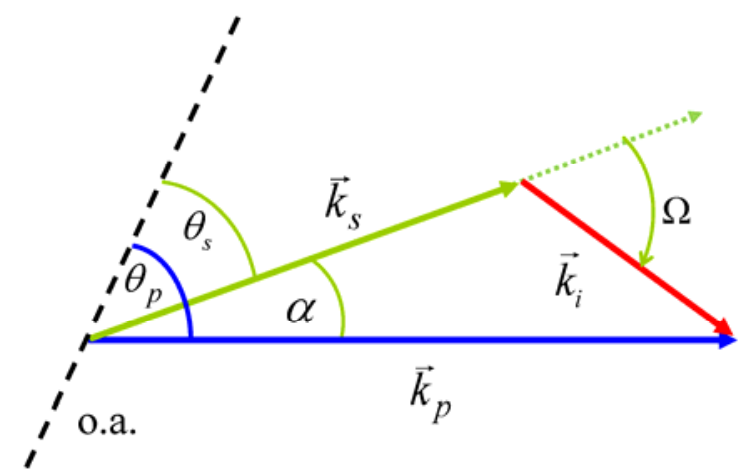

Figure 7.5. Noncollinear phase-matching angles and wave-vector distribution inside the nonlinear crystal. o.a.: optical axis.

In the case of a noncollinear configuration, such as for example that represented in Figure 7.5, the phase-matching condition becomes a vector equation, which, projected along directions parallel and perpendicular to the signal wave-vector, becomes:

$$
\begin{gathered}
\Delta k_{\|}=k_{p} \cos \alpha-k_{s}-k_{i} \cos \Omega=0 \\
\Delta k_{\perp}=k_{p} \sin \alpha-k_{i} \sin \Omega=0
\end{gathered}
$$

Note that the angle $\Omega$ is not fixed, but depends on the signal wavelength. We can consider, as we did before, that if the signal increases by $\Delta \omega$ the idler frequency decreases by the same quantity: $\Delta \omega$. We can make an expansion of the wave-vector mismatch in the two directions and obtain [127]:

$$
\Delta k_{\|} \cong-\frac{\partial k_{s}}{\partial \omega_{s}} \Delta \omega+\frac{\partial k_{i}}{\partial \omega_{i}} \cos \Omega \Delta \omega-k_{i} \sin \Omega \frac{\partial \Omega}{\partial \omega_{i}} \Delta \omega
$$




$$
\Delta k_{\perp} \cong \frac{\partial k_{i}}{\partial \omega_{i}} \sin \Omega \Delta \omega+k_{i} \cos \Omega \frac{\partial \Omega}{\partial \omega_{i}} \Delta \omega
$$

To achieve broadband phase matching, both $\Delta k_{\|}$and $\Delta k_{\perp}$ must vanish. Upon multiplying Eq. (7.11) by $\cos \Omega$ and Eq. (7.12) by $\sin \Omega$ and adding the results, we obtain the following equation:

$$
\frac{\partial k_{i}}{\partial \omega_{i}}-\cos \Omega \frac{\partial k_{s}}{\partial \omega_{s}}=0
$$

which is equivalent to:

$$
v_{g s}=v_{g i} \cos \Omega
$$

Equation (7.14) shows that broadband phase matching can be achieved for a signal-idler angle, $\Omega$, such that the signal group velocity equals the projection of the idler group velocity along the signal direction. Note that Eq. (7.14) can be satisfied only if $v_{g i}>v_{g s}$, which is the case in the commonly used Type I phase matching in negative uniaxial crystals, where both the signal and idler are under the influence of the ordinary refractive index.

Recall that from a more practical point of view it is more useful to know the pump-signal angle, $\alpha$, rather than $\Omega$, because it is in fact the angle that we can control experimentally.

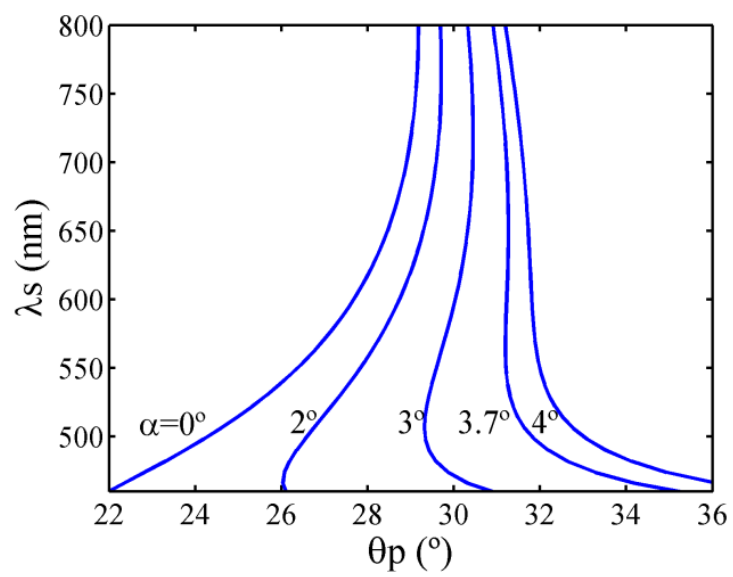

Figure 7.6. Phase-matching curves for a noncollinear Type I BBO OPA pumped at $400 \mathrm{~nm}$, for different pump-signal angles, $\alpha$.

This is the key to broadband parametric amplification; when the phase-matching bandwidth is maximized, the angle that the pump and signal waves form, which equals $3.7^{\circ}$ in the case of the $\mathrm{BBO}$, is called the magic angle. In the Figure 7.6 we have plotted the noncollinear phase-matching curves as a function of the phase-matching angle, $\theta_{\mathrm{p}}$, and the signal wavelength for different pumpsignal angles, $\alpha$. In the case of $\alpha=3.7^{\circ}$, note how for a fixed value of $\theta_{\mathrm{p}}$ most of the visible wavelengths in the signal (from 500-700 nm) are phase-matched at the same time. In other words, there is no need to rotate or tilt the crystal (angle tuning) to amplify one frequency or the other. Note the difference with the collinear case, $\alpha=0^{\circ}$, in which each pump angle corresponds to only one signal wavelength. 
In the next figure, we have represented the OPA gain for the particular case of $\alpha$ being equal to the magic angle in $\mathrm{BBO}$, while in panels $\mathrm{b}$ ) and c) we plot the gain at two different phase matchingangles, $\theta_{\mathrm{p}}$. Note how slight changes in the phase-matching angle significantly change the gain bandwidth.
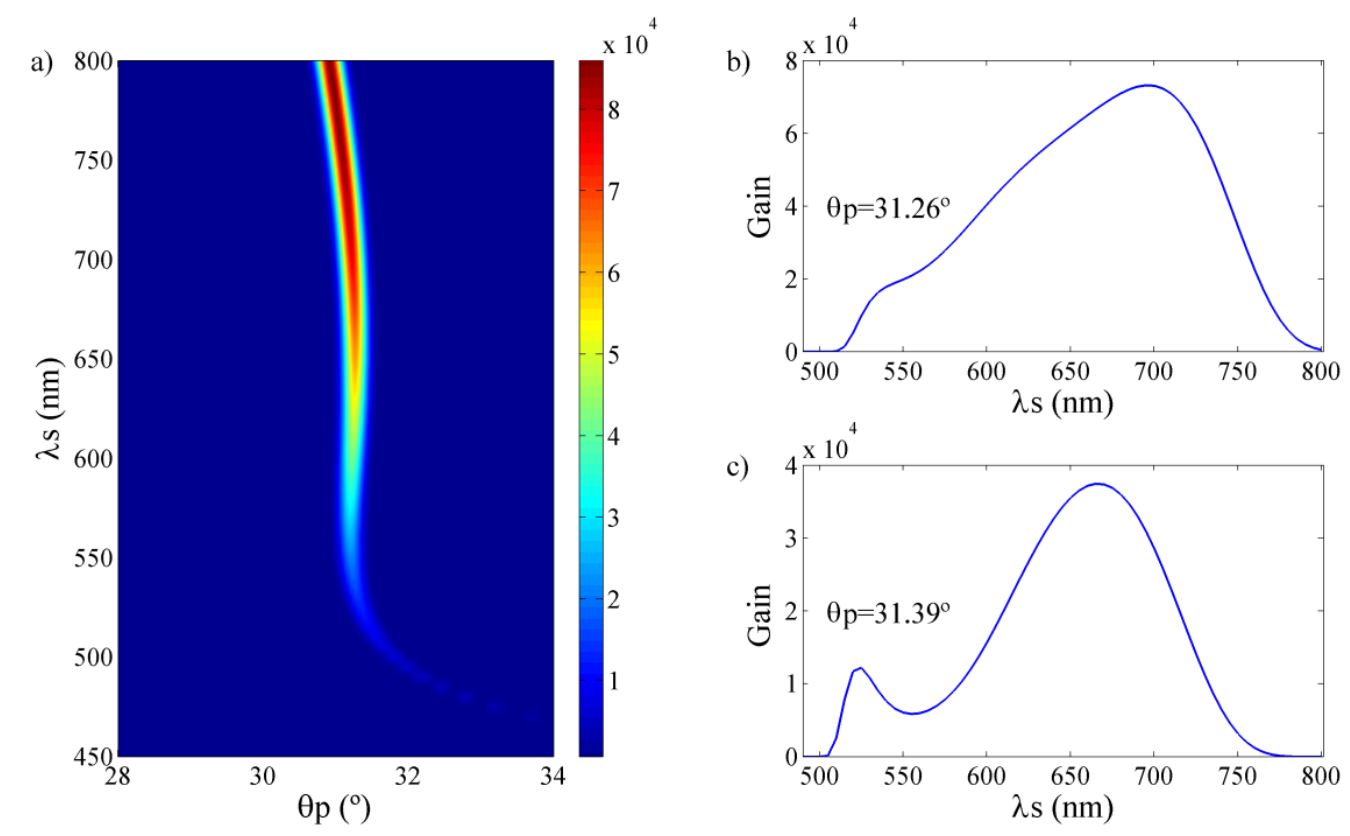

Figure 7.7. OPA gain calculated for a noncollinear Type I interaction in BBO, pumped by the second harmonic of the Ti:Sapphire laser. The internal pump-signal angle equals the magic angle, $\alpha=3.7^{\circ}$, for this crystal. The colourbar represents the gain factor in arbitrary linear units. b) and c) gain bandwidth at certain values of the phase matching angle $\theta_{p}$ indicated in each figure.

The next figure represents the normalized OPA gain for different crystal lengths in the case of phase-matching angle: $\theta_{\mathrm{p}}=31.26^{\circ}$. Note how the gain bandwidth decreases with increasing crystal length, $L$, as follows from Eq. (7.8). This behaviour is similar to that seen in the one seen in Sect. 5.3 for the phase-matching bandwidth of SHG.

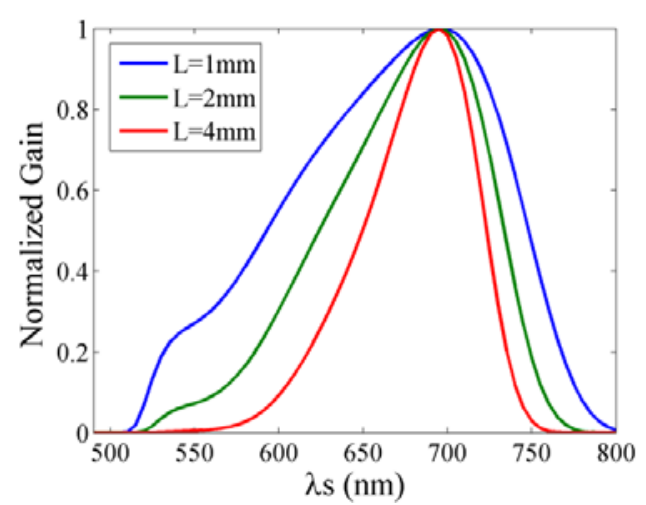

Figure 7.8. OPA gain bandwidth calculated for a noncollinear Type I interaction in BBO samples of different thickness, pumped by the second harmonic of the Ti:Sapphire laser. The internal pump-signal angle was equal to the magic angle $\alpha=3.7^{\circ}$ for this crystal and the phase matching angle was $\theta_{\mathrm{p}}=31.26^{\circ}$. 
This favourable property of noncollinear geometry for broadband parametric amplification was first recognized by Gale et. al. [129], although that case it was applied to OPOs. Wilhelm et al. were the first to demonstrate a noncollinearly phase-matched OPA pumped by blue pulses at a $1 \mathrm{kHz}$ repetition rate, which can readily yield sub-20 fs pulses, tunable throughout the visible region [130]. More examples of noncollinear visible OPAs can be found in [123, 130-132].

\subsubsection{OPG: the Route to Ultra-broad Gain Bandwidth}

The OPG process, also known as parametric superfluorescence, can be used to generate the signal that will be then amplified in an OPA stage [133]. In fact, this is the scheme used by some commercial OPA devices [134]. OPG is the parametric amplification of the spontaneous signal that is generated when only the intense pump pulse enters the nonlinear medium [135]. This process cannot be predicted by classical treatment of the interaction, because two monochromatic waves are needed as input to induce the nonlinear polarization oscillating at the frequency difference: see Sect. 2.4.2. Quantum mechanically, this process is described as a pump photon incident on a crystal that spontaneously splits into a signal and idler photons [136]. The signal-idler pair generated is determined by phase-matching conditions; therefore, the OPG bandwidth is the same as the phasematching bandwidth; for example the one in Figure 7.7 a). Precisely due to the phase-matching geometry, the emission is in form of a light cone. In other words, the signal and idler waves are propagating on conical surfaces having a common axis that coincides with the direction of propagation of the pump beam. The geometry of the beams inside the crystal is the one described in Figure 7.5, and in Figure $7.10 \mathrm{~b}$ ) a photograph of a projection of the superfluorescence cone on a paper screen is shown. In this case the emission is visible because the pumping was the second harmonic of the Ti:Sapphire laser.

a)

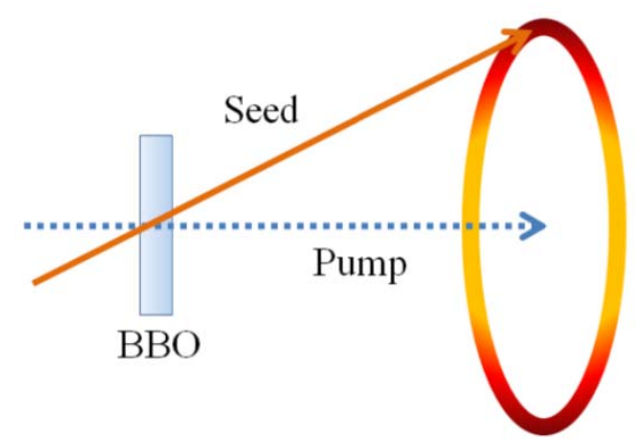

b)

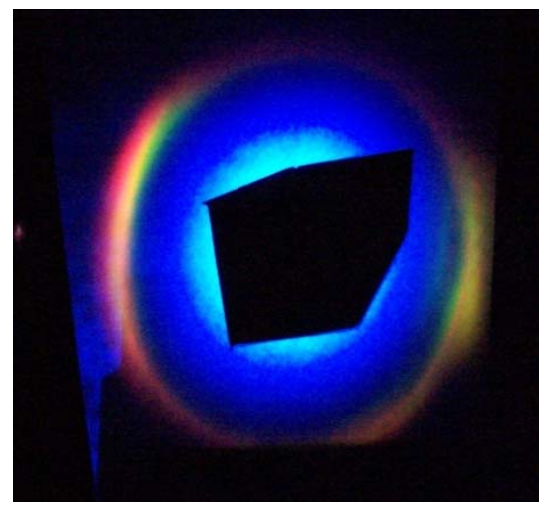

Figure 7.9. a) Sketch of the OPG process, indicating the injection of the seed beam. b) Image of the cone of superfluorescence projected on a white-paper screen. In the middle there is a hole to allow the intense blue pump to pass through the paper in order to avoid saturation of the CCD.

In order to generate this process experimentally, as there is no seed it is necessary to use a more intense pump than for OPA. In fact, the intensity needed $\left(80 \mathrm{GW} / \mathrm{cm}^{2}\right.$ in $\mathrm{BBO}$, for a $1.5-\mathrm{mm}$ thick sample [135]) is close to the damage threshold of the nonlinear crystals. This phenomenon is important in noncollinear OPAs because it is used to adjust the pump-signal angle and to obtain broadband phase matching. In practice, the pump-signal angle is made to coincide with the apex angle of the superfluorescense cone, as indicated in Figure 7.9 a). This is the direction for which 
group-velocity matching between signal and idler is guaranteed; i.e. condition (7.14) is fulfilled. Under these conditions an ultra-broadband gain bandwidth extending over most of the visible region was observed [123].

\subsection{Experimental Set-Up: the NOPA in Salamanca}

In this section we shall describe the experimental set-up of the NOPA that was developed in Salamanca. The system had some similarities to the ones described in $[124,137]$. A sketch of the set-up is shown in the Figure 7.10. As already mentioned in Chapter 3, half of the energy from the regeneratively amplified Ti:Sapphire laser is divided between the micro-processing laboratory and our experimental table where the OPA set-up was built. Therefore, the total energy available was $\sim 0.5 \mathrm{~mJ}$, from which we took a sample with a beam sampler (90/10). The low-energy part went into the seed arm, i.e. to the SC generation stage, even though the energy was still too high to be focused on the sapphire or fused silica plate. As mentioned in the previous chapter, the energy suitable for SC generation is in the few $\mu \mathrm{J}$ s regime. Therefore, we reduced it with an energy attenuator (a half-wave plate and a linear polarizer). Also, an iris was interposed in front of the focusing lens ( $\mathrm{L} 1, \mathrm{f}=30 \mathrm{~cm}$ ) to increase stability when necessary. After being focused in the plate, the SC beam, (indicated with the multicolour line in the Figure 7.10) was collimated with another lens $(\mathrm{L} 2, \mathrm{f}=15 \mathrm{~cm})$, and was finally focused $(\mathrm{L} 3, \mathrm{f}=40 \mathrm{~cm})$ in the first BBO crystal, labelled OPA 1 , which was the first amplification stage.

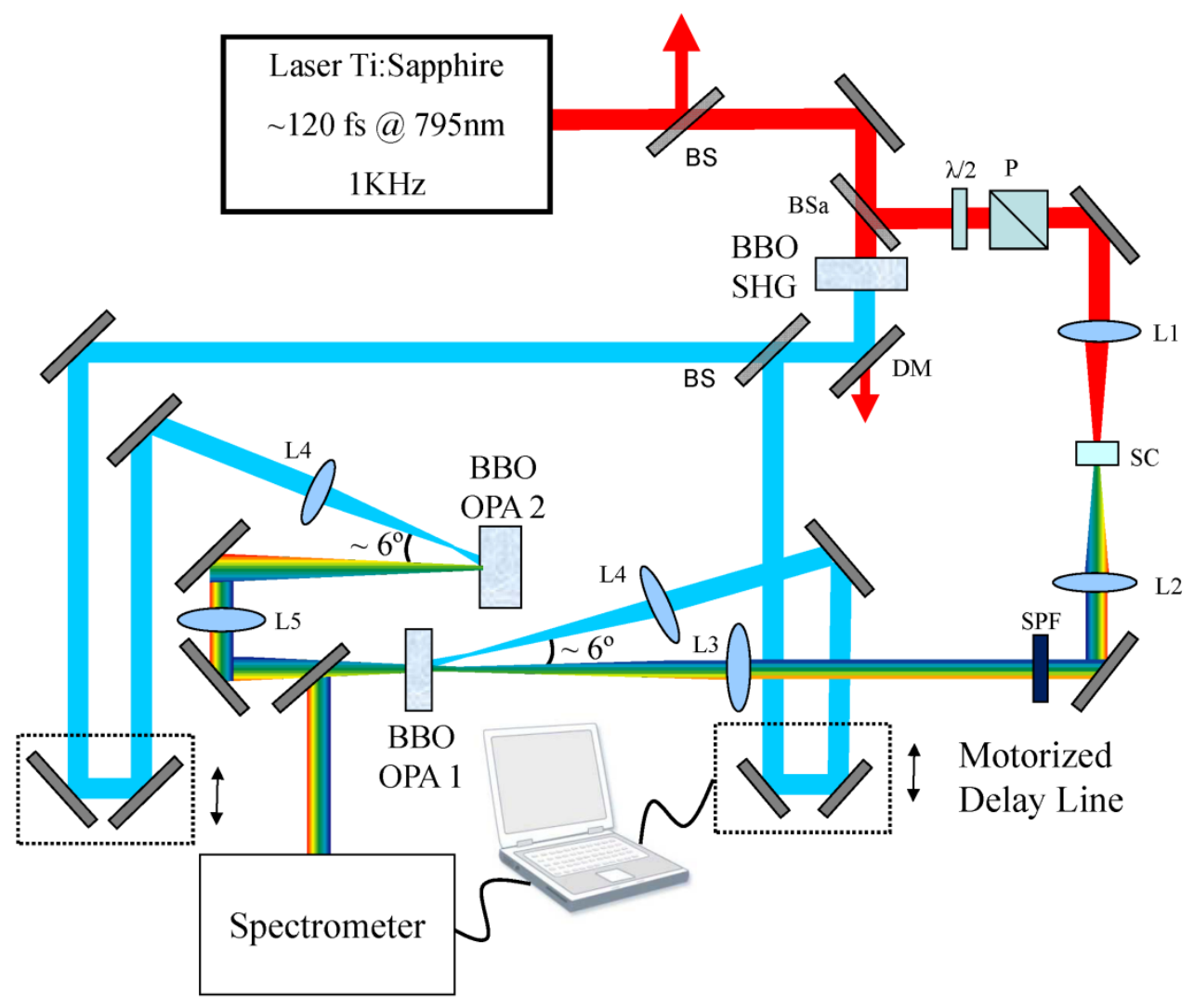

Figure 7.10. Sketch of the experimental set-up of the two NOPA stages in the visible. BS: Beam-Splitter, BSa: beam sampler, P: polarizer, SPF: short-pass filter, DM: dichroic mirror, L: lens, SC: sapphire or fused silica plate for SC generation. 
The remaining part of the fundamental beam (the $90 \%$ ) was frequency-doubled in a $14 \times 14 \times 0.5 \mathrm{~mm}^{3}$ BBO crystal: "BBO SHG" in the figure $\left(\theta=29.4^{\circ}, \phi=90^{\circ}\right)$. The pulses generated served as a pump for the two NOPA amplification stages. Therefore, after the nonlinear crystal a 50/50 beamsplitter was used to split the energy between the two beams equally. Prior to being sent to the beamsplitter we used a dichroic mirror to separate the second harmonic from the unconverted infrared. Also, a band-pass filter (FGB37s, Thorlabs), not included in the figure, was used to block the remaining IR signal. Both second-harmonic beams travelled to a delay line and were focused (L4, $\mathrm{f}=20 \mathrm{~cm}$ ) into each OPA crystal. The delay lines are required to temporally overlap the pulses in time, and compensate the differences in the beams' paths. Recall that optical amplification can only occur if the pump and seed light pulses overlap in both space and time.

The amplified signal obtained in the first amplification stage was then focused (L5, $\mathrm{f}=20 \mathrm{~cm}$ ) in the second amplification stage. Also, after the first amplification stage we interposed a spectrometer for the characterization process, as described in Sect. 7.6.

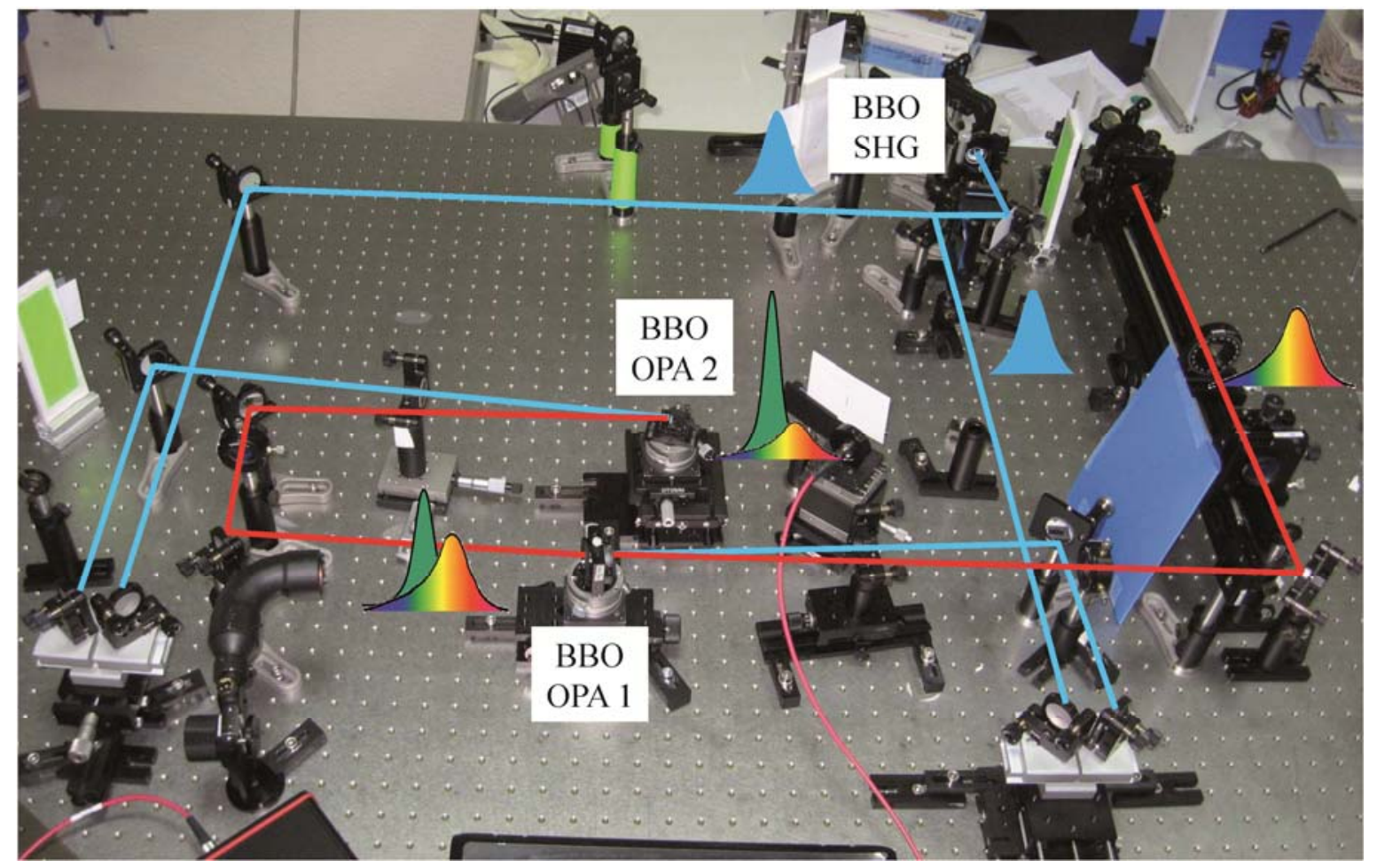

Figure 7.11. Photograph of the two-stage NOPA in the visible.

We chose a noncollinear interaction, because as commented this offers ultra-broadband amplification. The internal magic angle $\alpha=3.7^{\circ}$ becomes an external angle of $\sim 6.4^{\circ}$, also indicated in Figure 7.10. The OPA 1 and OPA 2 are BBO crystals cut for SHG of the fundamental $\left(\theta=29.4^{\circ}\right.$ and $\phi=90^{\circ}$ ), with sizes of $6 \times 6 \times 1 \mathrm{~mm}^{3}$ and $6 \times 6 \times 2 \mathrm{~mm}^{3}$ respectively. As can be seen in Figure 7.6 the phase-matching angle $\theta_{\mathrm{p}} \sim 31^{\circ}$ that corresponds to the pump-signal magic angle is close to the phase-matching angle for SHG of the fundamental at normal incidence $\left(\theta=29.4^{\circ}\right)$. Therefore, a slight tilting of the crystal $\sim 2^{\circ}$ was needed in order to phase match the OPA interaction properly. This is detrimental to the efficiency of the process, because part of the pump and signal pulse energy will be reflected in the nonlinear crystal. 
Regarding the size of the OPA crystals, in Chapter 2 we discussed how the parametric gain increased with crystal length. However, in Figure 7.8 we show how the gain bandwidth decreases with increasing crystal length. Therefore, a compromise between the amplification factor and the amplified bandwidth must be found. The solution taken in most OPA systems is to use multiple amplification stages instead of a single thick crystal [89]. This decision has a dual basis: on one hand it is possible to vary the pump intensity separately, and as a result adjust the gain in each stage. On the other the GVM between the pump and signal pulses in the first nonlinear crystal can be compensated by the delay line in the second crystal.

\subsubsection{Experimental Results}

Regarding the experimental results, we shall discuss the spectra of the signal pulses obtained after two amplification stages in the NOPA (see Figure 7.12). From the arguments given above, one may expect a signal spectrum covering almost all the visible region. In contrast, the image shows not very broad spectra centred at different wavelengths in the visible region. Note that in the discussion above we have not taken into account how the temporal duration of the two pulses might affect the bandwidth of the amplified pulse. If the pump and the signal pulses have the same temporal duration the full bandwidth predicted in Sect. 7.4.1 could be amplified, although this was not the case in our optical set-up.

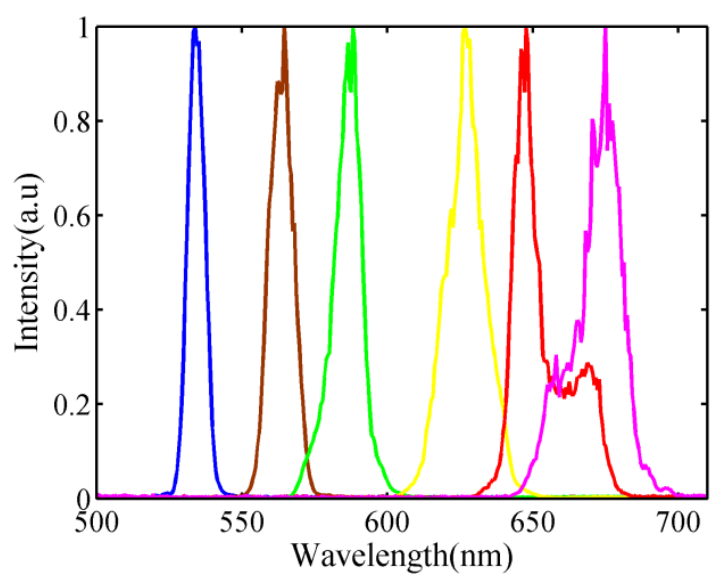

Figure 7.12. Amplified spectra, after two amplification stages in a blue-pumped NOPA. The different spectra were obtained by changing the relative delay between seed and pump pulses for a fixed position of the crystal. The spectra have been normalized.

We expected that the SC pulses might have been lengthened due to dispersion, after travelling through the different materials in the set up, from the sapphire or fused silica plate itself, to the focusing lenses. Therefore, the pump pulses were expected to be much shorter than the SC; owing to their lower wavelength content, they should have been less affected by dispersion. We expected a pulse duration of $\sim 130 \mathrm{fs}$, compatible with the X-FROG measurements in Chapter 3. The consequence of this difference in the temporal duration of the two pulses is that only portions of the SC will be temporarily overlapped with the pump, and therefore only those parts can be amplified each time. Thus, since the phase-matching bandwidth was broad, tunability was achieved by changing the relative delay between the two pulses; in other words, the pump pulse was overlapped with different "slices" of the SC, and the central wavelength was selected without the need to tilt or rotate the crystal. 
Although in our case the latter described effect is precisely what afforded us the possibility of easy wavelength tuning, simply by changing the pulse relative delay, this is detrimental when OPAs serve to generate pulses of much lower temporal duration than the original ones. This is because for a fixed delay it is not possible to amplify the whole bandwidth available. Moreover, the high dispersion introduced in the signal should hinder the chirp compensation in a subsequent compression stage, which was not included in our set-up.

The NOPA pulse bandwidth can be optimized by controlling the chirp of the white-light seed to fit its temporal width with that of the pump pulses under an optimal alignment (note that this condition was achieved by adjusting the pump-signal angle to match the apex angle of the superfluorescence cone). Under these optimized conditions, a typical amplified spectrum extends over an intensity FWHM bandwidth of $(\sim 250 \mathrm{~nm})$ and is hardly tunable at all since it covers the maximum available gain bandwidth [123].
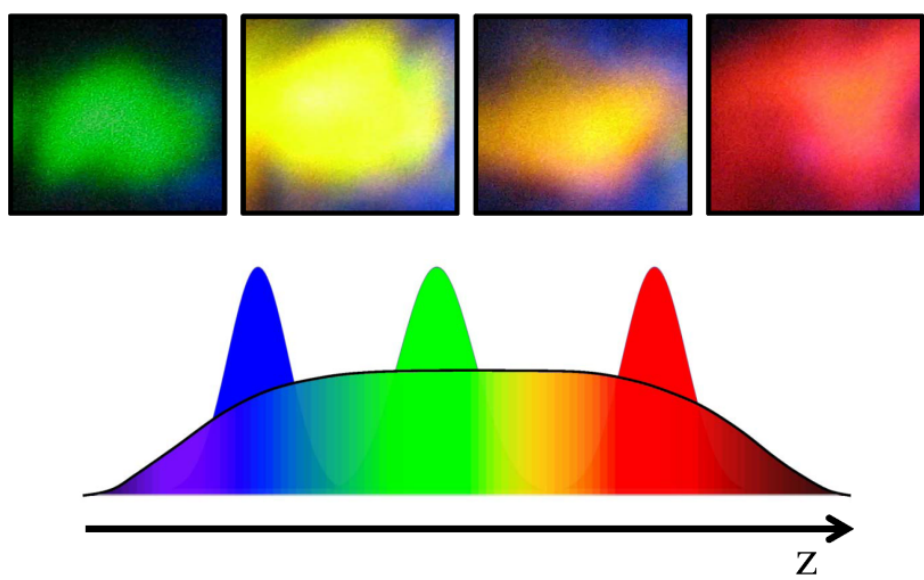

Figure 7.13. Photographs of the amplified signal beams projected on a screen (top). Sketch representing the SC pulse and the different portions amplified for each delay.

In Table 7.1 we include the value of the bandwidth ( $\Delta \lambda$, FWHM in intensity) along with the corresponding Fourier-transform limit of the pulses from Figure 7.12. Note that even though the system is not optimized for compensation of dispersion, the amplified pulses could be compressed to a duration much shorter than the fundamental pulses.

\begin{tabular}{c|cccccc}
\hline & Blue & Brown & Green & Yellow & Red & Purple \\
\hline$\lambda_{\mathbf{c}}(\mathbf{n m})$ & 534 & 564 & 588 & 626 & 647 & 675 \\
$\Delta \lambda(\mathbf{n m})$ & 7 & 9.8 & 9.7 & 12 & 8.6 & 11.9 \\
FL (fs) & 60 & 48 & 52 & 48 & 72 & 55 \\
\hline
\end{tabular}

Table 7.1. Corresponding central wavelength, intensity FWHM spectral bandwidth, and temporal Fourier Limit (FL) of the pulses shown in Figure 7.12.

In addition we took snapshots of the beams projected on a white screen (Figure 7.3). These measurements corresponded to the preliminary results and the shape of the beams was not very good. In this case the size of the seed and pump beams were not so similar, the pump being smaller, such that a good transfer of energy from the pump to the signal was not possible. 
A measure of the energy in the different amplified pulses in Figure 7.12 is included in the next figure. The gain factor was calculated as the output energy after the second amplification stage divided by the energy in the first amplification stage.

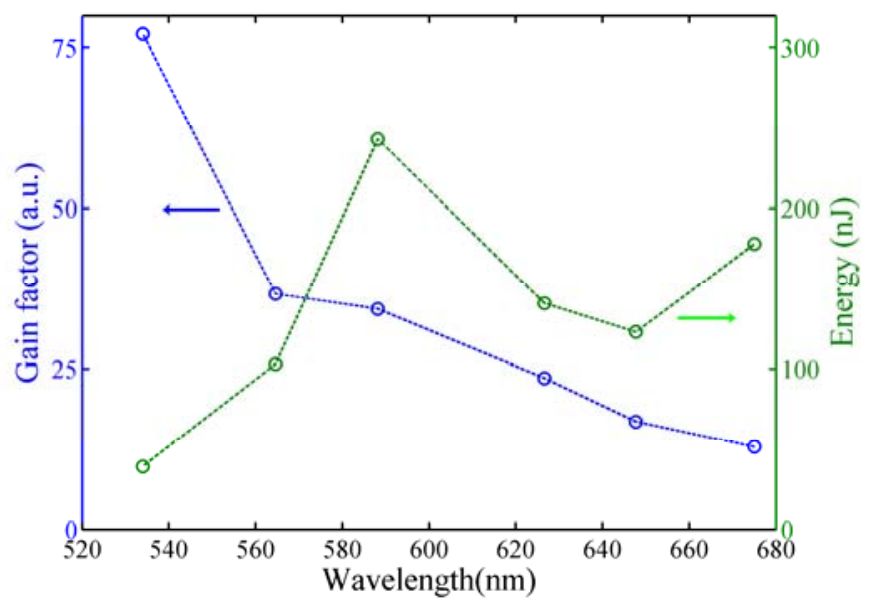

Figure 7.14. Representation of the gain factor in the second NOPA stage for the OPA signals whose spectrum is included in Figure 7.12. The pulse energy is included on the right axis.

Even after two amplification stages, the energy is low and therefore our next logical step should be to improve the gain factor of the NOPA (we plan to do this in future implementations). In this last part of the section we shall comment on the issues regarding the pump energy which we consider to be responsible for this low energy output. As we have explained at the beginning of Sect. 7.4, of the $\sim 400 \mu \mathrm{J}$ energy accessible to this experiment $90 \%$ is dedicated to the pumping, and it is first doubled in the $0.5-\mathrm{mm}$ thick crystal. However with the unfocused beam the peak intensity in the fundamental $\left(\sim 6 \mathrm{GW} / \mathrm{cm}^{2}\right)$ is low and the conversion efficiency in the SHG crystal is consequently very low: approximately 7\% (see Figure 3.9 a)). The measured second harmonic pulse-energy available in each amplification stage was $12 \mu \mathrm{J}$. This energy is not sufficient for pumping the OPA with the beam unfocused, since the corresponding peak intensity is 0.17 $\mathrm{GW} / \mathrm{cm}^{2}$. We decided to focus the pump beams in order to achieve an adequate level of intensity; another option would have been to reduce the beam size by using a telescope, but the extreme reduction rate needed makes this inadvisable.

One of the problems with focusing the pump is that the focal spot should not be inside the nonlinear crystal because damage may occur easily. In fact, the beam size with the lens used ( $\mathrm{f}=20$ $\mathrm{cm}$ ) had a theoretical radius of $5 \mu \mathrm{m}$ (at $1 / \mathrm{e}^{2}$ intensity criterion), which results in a peak intensity of $\mathrm{I}_{0}=1.6 \times 10^{14} \mathrm{~W} / \mathrm{cm}^{2}$ (fluence of $30 \mathrm{~J} / \mathrm{cm}^{2}$ ). This intensity is large enough to produce strong-field ionization in the crystal. As a reference, typical ablation fluences are $1 \mathrm{~J} / \mathrm{cm}^{2}$ at this wavelength in many crystals [138].

The solution we took was to work at a certain distance behind the focus of the lens. In the next figure we plot the relative decrease in pump intensity $\mathrm{I}(\mathrm{z}) / \mathrm{I}_{0}$ as a function of the distance to the focus, $\mathrm{z}_{\mathrm{f}}$. In the lens that focused the pump we included a linear stage so that we could finely vary the distance between the lens and crystal. Note that at the same time we were changing the size of the beam, as indicated in Figure 7.15 b). The latter also has an implication in the efficiency of the 
process, because the ideal situation would be for the signal and the pump to have the same size. Therefore the signal beam size should be changed at the same time.

a)

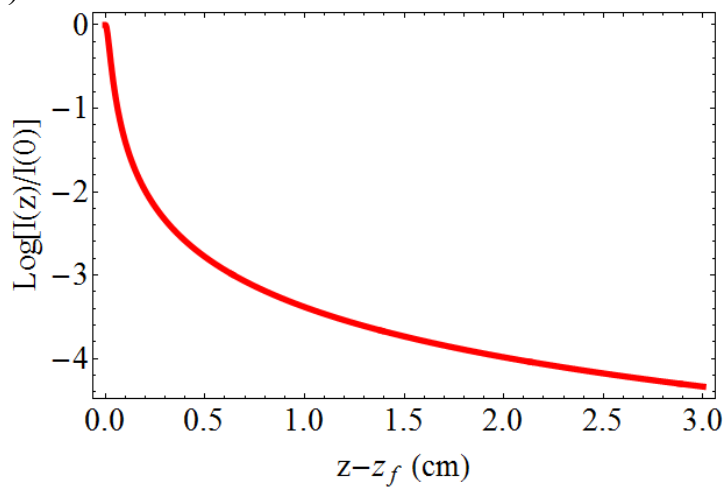

b)

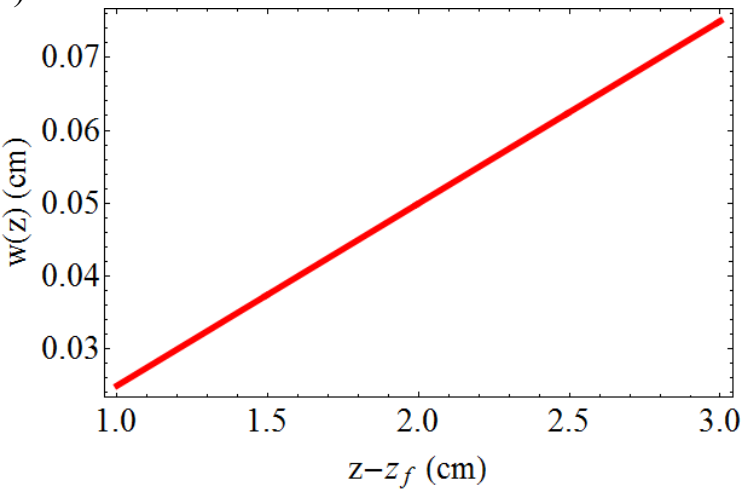

Figure 7.15. Variation in a) the pumping intensity and b) pump-beam radius with the distance to the lens focal point.

In order to operate safely we worked at a large distance from the focus, between $\mathrm{z} \sim 2 \mathrm{~cm}$ and $\mathrm{z} \sim$ $1.2 \mathrm{~cm}$, with corresponding theoretical peak intensity in the range of $\sim 15-30 \mathrm{GW} / \mathrm{cm}^{2}$. Our intention was to perform new measurements of the gain factor, trying to improve the results of Figure 7.14. If we remember the calculation of the Figure 2.12, in case of the $2 \mathrm{~mm}$ crystal the parametric gain corresponding with this intensity range was $\sim 10^{5}$. However this value is "huge", and we did not expect to obtain such high gain factor. Several issues prevent us to achieve the theoretical value. To start with, the input intensities are theoretical calculations, the real intensity is expected to be lower, owing for example to an irregular beam shape. Moreover, we are not taking into account the losses by reflexion in the crystal. In addition the pump beam is divergent; therefore the intensity is not constant all along the crystal, as is supposed in the theoretical model. Regarding the OPA process itself, the fact of working with pulses instead of a continuous-wave beam, will also decrease the gain value, as the temporal superposition of the pulses cannot be maintained along the crystal. Moreover, and perhaps more importantly, we are working in a noncollinear configuration, which is detrimental for the spatial overlap between the interacting pulses.

\subsubsection{Measurement of Idler Spectra}

We measured the spectra of some of the idler beams generated in the NOPA. As can be seen in Figure $7.1 \mathrm{a}$ ), the idler wavelengths that will be generated correspondingly with the amplified signal in the visible region, are mostly in the IR, and the detection of the spectrometer available in the laboratory scarcely reaches $1.1 \mu \mathrm{m}$. However, lower wavelength idler signals have been measured, and are shown in Figure 7.16 a). The idler has the same frequency bandwidth as the signal and can therefore potentially be very short. However, the idler signals have the problem of being spatially chirped, i.e. due to the geometry of the interaction. The emission angle of the idler beam, $\Omega$, is wavelength dependent as follows from Eq. (7.10). Each idler wavelength emerges at a different angle from the nonlinear crystal, and hence the beam is subject to large angular dispersion [139].

However, more surprising for us was the discovery of wavelengths in the visible region, which due to the location of the fiber could not belong to the seed beam. Actually, what we had measured was the second harmonic of the idler beam. Figure $7.16 \mathrm{~b}$ ) represents the measured spectra of the second harmonic of the idler centred at $850 \mathrm{~nm}$, which is represented in blue in Figure $7.16 \mathrm{a}$ ). The 
central wavelength of the second-harmonic spectrum is centred at $425 \mathrm{~nm}$ : exactly half of the central wavelength of the idler. Also, the intensity FWHM bandwidth of the second harmonic (8 $\mathrm{nm})$ is half that of the IR pulse. The phase-matching angle to double this idler $\left(27.6^{\circ}\right)$ proved to be the angle (with respect to the optical axis) of emission of the idler beam. In fact, since the signal wavelengths were the same as the second harmonic of the idler wavelengths, the interference of these two beams (the signal and the second harmonic of idler) proved to be a means to measure the pulse to pulse carrier-envelope phase drift of the signal beam [139], as is done in a typical $f-2 f$ interferometer.

a)

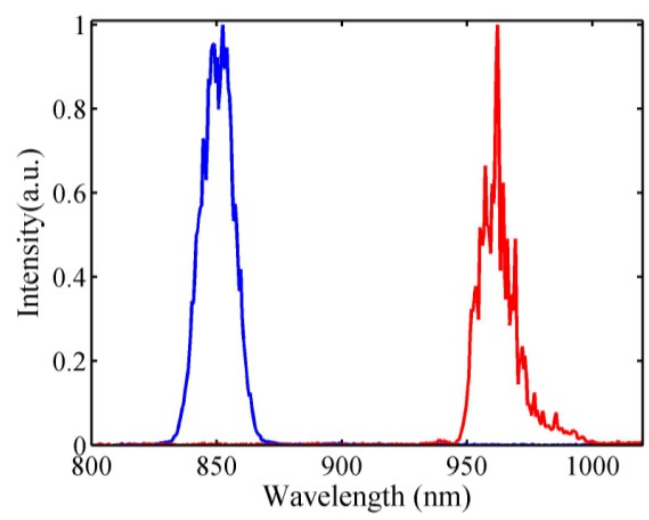

b)

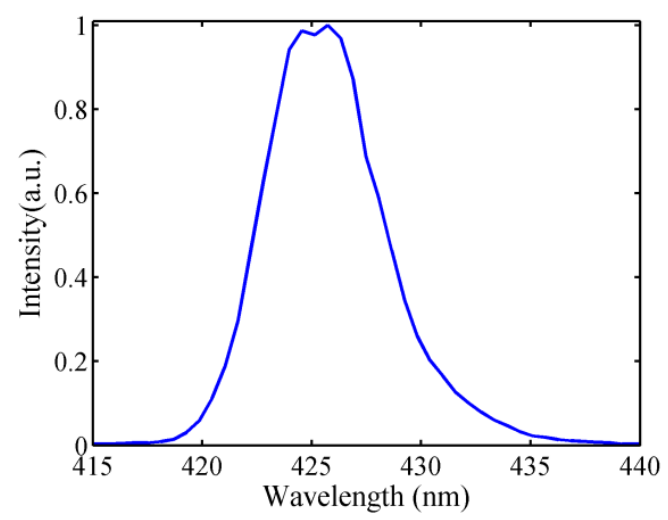

Figure 7.16. a) Spectra of idler beams generated after the first amplification stage. b) Spectra of the second harmonic of the idler.

\subsection{Characterization of SC Pulses}

In a similar way to the case of the SHG/THG module in Chapter 2, the first amplification stage of the NOPA set-up served us to characterize the SC pulses, generated either in sapphire or fused silica plates. For this purpose we again chose a cross-correlation technique. In this case, the reasons why it is not possible to characterize the SC pulses by a self-referred technique, such as autocorrelation, are mainly two: on the one hand the SC signal is very weak, so the efficiency of the SHG process might be very low. On the other hand, it is very difficult to find crystals that can phase-match the broadband spectra and if they exist, they should be very thin, so efficiency would prove to be even lower. Therefore, as we shall show, the OPA process is perfect for characterizing them. However, it should be recalled that there are two differences with the process described in Chapter 3. One has to do with the nonlinear process that generates the signal to be analyzed: in this case the one involved is the DFG process: more precisely the OPA. Secondly, the signal that will be detected is the amplified seed, instead of the idler, which is the one generated in the process. Recall that in the case of the SFG-based X-FROG we detected the UV signal, which was the resultant sum of the two interacting pulses: the second harmonic and the fundamental. Moreover, in this case the test/gate pulses will be the second-harmonic pulses generated in the first BBO, and not the fundamental ones as in the above case.

Figure 7.17 a) shows a typical X-FROG trace obtained for the characterization of the SC generated in a 1-mm thick sapphire sample. In fact we are characterizing only the part of the SC that can be amplified in the visible NOPA, and with the broad phase matching bandwidth (see Figure 7.6 with $\alpha=3.7^{\circ}$ ). The remaining IR part of the SC pulses is removed before the BBO crystal with the short-pass filter (FES0750, Thorlabs). The same reason why it is possible to tune the 
central wavelength of the amplified signal, changing the delay position, is what allows these measurements to be made. In order to obtain the trace we have recorded a spectrum in each different delay position, where the delay zero is considered to be when the blue/pump pulses are overlapped with the leading part of the SC pulses, in particular it corresponds with the cut-off wavelength of the filter $(750 \mathrm{~nm})$. Recall that there is no frequency conversion in this case and only the selective amplification of subsequent parts of the SC pulse.

The same kind of trace was recorded using a fused silica plate (6-mm thick) to generate the SC pulses. Figure 7.17 b) shows an example of one of them. If we compare the two traces, we can see how the seed pulses proved to be much longer in the case of the fused silica.
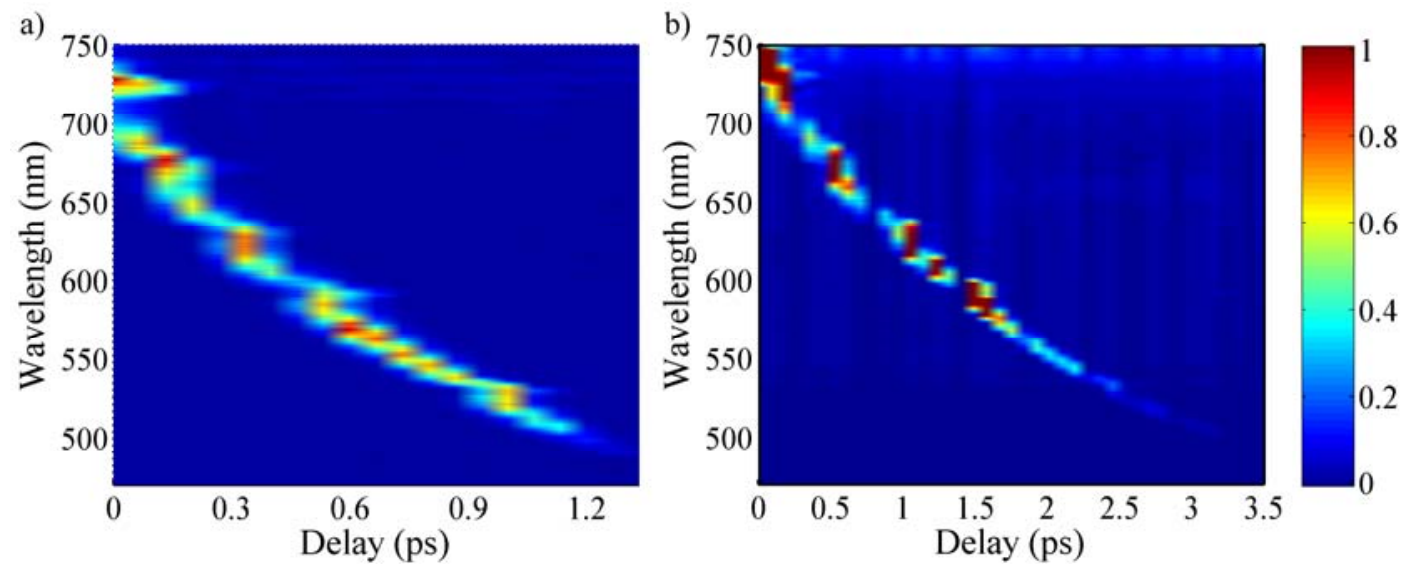

Figure 7.17. a) Experimental OPA X-FROG traces obtained to characterize the SC generated in a) a 1-mm thick sapphire sample and b) a 6-mm thick fused silica plate.

The difference in these traces with those obtained in previous chapters is evident. The fact that the SC pulses are much longer in time than the gate/pump pulses means that it is not necessary to use a retrieval algorithm in order to obtain information about the SC pulses, such as the instantaneous frequency. In this case, the pump pulses act as a narrow gate directly providing the variation in frequency with time within the pulse. This characteristic can be seen more clearly in Figure 7.18 a) where we have plotted the peak wavelength of each spectrum obtained from the trace, in front of the delay. Having information about the instantaneous frequency is unusual in many X-FROG measurements [140] where the unknown and gate pulses have a similar temporal durations.

The vertical lines in Figure 7.18 a) show the expected bandwidths of the pulses generated for three different values of the relative delay. We can see how the bandwidth increases with increasing peak wavelength. This effect is again a consequence of dispersion; it is simply the result of superposing the pump pulses with different portions of the SC. Note how the slope of the instantaneous frequency increases in the red part of the spectrum. Moreover in order to calculate this bandwidth we have also taken into account the effect of GVM, leads the blue pulse to become overlapped to slightly different regions of the $\mathrm{SC}$ pulse during its propagation inside the $\mathrm{BBO}$, for a given delay. This effect is analogous to the time-smearing effect described in Sect. 4.5, which artificially elongates the cross-correlation traces. This effect of the GVM is also more important when the delay is such that the pump is overlapped with the redder frequencies in the SC owing to a more difference in their group velocities. In this case, the delay between the pump and signal along the crystal is $\sim 160 \mathrm{fs}$, slightly higher than the temporal duration of the pump. 
a)

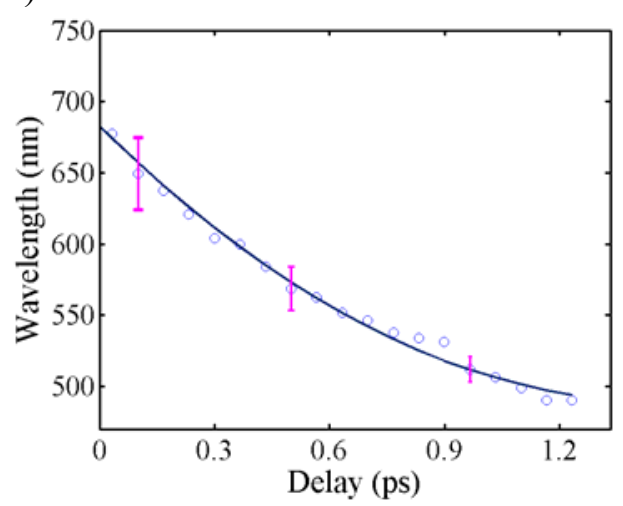

b)

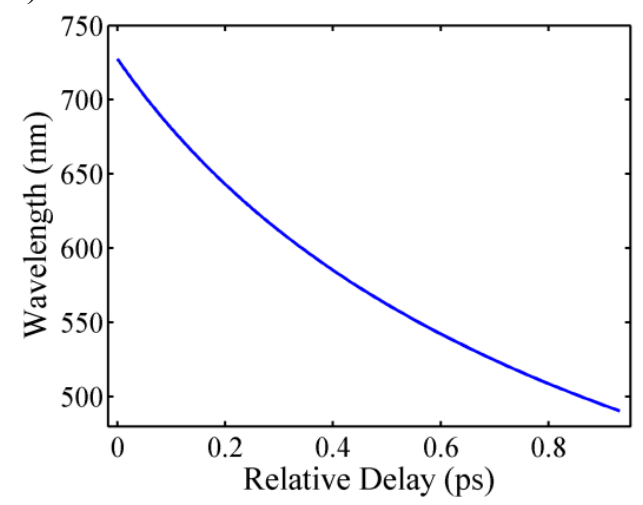

Figure 7.18. a) Peak wavelengths of the spectra in Figure 7.17 a) as function of the delay -open circles-. The vertical pink lines delimit the expected bandwidth and the blue line represents the adjustment to a second order polynomial. See the text for a detailed explanation. b) Relative delay between the different wavelength components of the SC pulses, generated in a sapphire plate of 1-mm thickness (see Eq.(7.17)).

As mentioned, given the special shape of the trace we can obtain useful information about the SC pulses. We start by taking the definition of the $\omega_{\text {inst }}(t)$, i.e. the temporal derivative of the pulse phase: $\omega_{0} t-\phi(t)$, where $\omega_{0}$ is the carrier frequency [42] and $\phi(t)$ is an unknown time-dependent phase. In order to make a an estimation of this phase $\phi(t)$ we can make an adjustment of the data of the $\omega_{\text {inst }}(t)$ to a second-order polynomial, -blue line in the Figure 7.18 a)-as:

$$
\omega_{i n s t}(t)=\omega_{o}-\frac{d \phi(t)}{d t} \approx \omega_{o}+a_{1} t+a_{2} t^{2}
$$

The coefficients from the adjustment give:

$$
\omega_{o}=2.56 \mathrm{rad} \cdot f s^{-1}, \quad a_{1}=0.001 \mathrm{rad} \cdot f \mathrm{~s}^{-2}, \quad a_{2}=-2.3 \times 10^{-7} \mathrm{rad} \cdot f \mathrm{~s}^{-3}
$$

The previous equation (7.15), can easily be integrated to obtain the temporal phase of the pulse:

$$
\phi(t)=-\frac{a_{1}}{2} t^{2}-\frac{a_{2}}{3} t^{3}=-5 \times 10^{-4} \frac{\mathrm{rad}}{\mathrm{fs}^{2}} t^{2}+7.6 \times 10^{-8} \frac{\mathrm{rad}}{\mathrm{fs}^{3}} t^{3}
$$

Concerning the temporal envelope of the SC pulse, it can be obtained in a rough estimation from the spectrogram (Figure 7.17 a)) by integrating it along the wavelengths dimension. To this end, it must be assumed that the phase matching is perfect for all the spectral bandwidth of the SC pulse and the gain factor is independent on the wavelength. Under these approaches, the retrieved intensity envelope and temporal phase is depicted in Figure 7.19.

As it can be seen in the retrieved pulse, the intensity drops from the leading to the trailing part, that is, from the IR to the UV wavelengths. It should be noted that the unconverted part of the original $795-\mathrm{nm}$ pulse is expected to travel in the leading part of the pulse but it is removed by the short-pass filter. The temporal phase along the pulse is huge, as is expected from the strongly chirped trace of Fig. 7.17. 


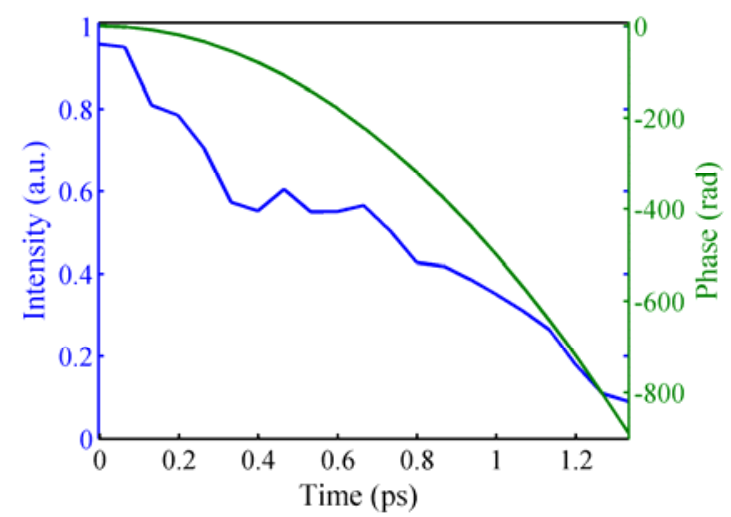

Figure 7.19. Retrieved intensity and phase of the SC pulse generated in a 1-mm thick sapphire sample.

The origin of the strong chirp is expected to be caused by dispersion in the different materials that the pulse propagates through. In order to confirm that, we compute the delay accumulated between each wavelength of the spectrum and the maximum value $(730 \mathrm{~nm})$. Thus, we evaluate:

$$
\tau(\lambda)=G V M \cdot L=\left(\frac{1}{v_{g}(\lambda)}-\frac{1}{v_{g}\left(\lambda_{\max }\right)}\right) \cdot L
$$

for all the optical materials of a given thickness $L$. We assume that all the wavelength components of the SC pulses are created at the same time just at the entrance of the sapphire plate, and from this point on, the propagation is linear. The materials that are taken into account are the $1 \mathrm{~mm}$ of sapphire, the focusing lens and the short-pass filter. The dispersion of the filter is unknown but we consider it as fused silica because the substrate is a silica-based glass. The delays are summarized in Table 7.2 and the final curve is shown in Figure $7.18 \mathrm{~b}$ ). The delay and its spectral dependence is consistent with the duration of the SC estimated in the X-FROG measurements.

\begin{tabular}{ccccc}
\hline Sapphire $(1 \mathrm{~mm})$ & BK7 $(4 \mathrm{~mm})$ & SF2 $(2 \mathrm{~mm})$ & $\begin{array}{c}\text { Fused silica } \\
(3 \mathrm{~mm})\end{array}$ & $\begin{array}{c}\text { Total delay } \\
\tau(\mathrm{fs})\end{array}$ \\
\hline $115 \mathrm{fs}$ & $464 \mathrm{fs}$ & $355 \mathrm{fs}$ & $199 \mathrm{fs}$ & $1133 \mathrm{fs}$ \\
\hline
\end{tabular}

Table 7.2. Delay acquired between two wavelength components in the SC pulses $(728 \mathrm{~nm}$ and 490) when travelling through the different materials in the set-up.

In summary, with our measurements, on the one hand, we can have a good approximation of the time duration of the SC pulses and on the other, we can attribute the lengthening of the SC to the dispersion acquired just by travelling through the materials in the set-up, as our estimations seem to be compatible with this.

\subsection{Second Experimental Set-Up: the NOPA in Bordeaux}

A similar set-up to the one previously described but with some improved features was implemented at the Centre Lasers Intenses et Applications (CELIA) laboratory in Bordeaux [141] during a research stay in that institution. In fact, the set-up was very similar to the one reported by [123], but without the post-compression stage. The main objective of building this OPA was to use it to amplify SC pulses but with the peculiarity of using a monochromatic source as pump. This unconventional source should be developed following a scheme similar to those described in [142, 
143]. The expected benefit of using a monochromatic source was that, in principle, it should be possible to amplify the whole SC, thus obtaining a broad bandwidth that would enable very short amplified pulses to be achieved. However, the implementation for the monochromatic source was difficult to obtain in the laboratory, mainly for technical issues, and the research objectives were changed accordingly. However, it is interesting to offer a description of the OPA built, as we shall see.

The laser system was different from the one in Salamanca, although it was also a Ti:Sapphirebased laser with CPA. The duration of the pulses was $\sim 40 \mathrm{fs},(\sim 20-\mathrm{nm}$ bandwidth, at intensity FWHM) with an energy per pulse of $5 \mathrm{~mJ}$, at a repetition rate of $100 \mathrm{~Hz}$. A sketch of the NOPA setup is shown in the Figure 7.20. It consists of only one amplification stage. From the sketch, it would be expected one can expect that the system would be more compact than the one in Salamanca, as indeed was the case.

A 90/10 beam sampler, was used to split the energy between the second harmonic and the SC generation stage. In this case the $90 \%$ was taken in reflection, in order to avoid the introduction of dispersion prior to the SHG stage. In this case the IR pulses were much shorter than those in Salamanca and any amount of material would be sufficient to observe the effects of dispersion. Moreover, the beamsplitter available was not adequate for working with ultrashort laser pulses since it was $\sim 1$-cm-thick.

In this case the BBO for SHG $\left(\theta=29.4^{\circ}, \phi=0^{\circ}\right)$ was $300-\mu$ m-thick in order to match the bandwidth of the laser (see Figure $3.8 \mathrm{~b}$ )). The blue pulses were then focused with a lens (L2, $\mathrm{f}=20$ $\mathrm{cm}$ ) in the BBO OPA. In the SC generation stage, we used a lens with a focal length of $15 \mathrm{~cm}$; then, two spherical mirrors, M1 and M2 with respective focal lengths of 15 and $30 \mathrm{~cm}$, were used to focus the $\mathrm{SC}$ in the BBO OPA, which in that case was a 1 -mm-thick sample $\left(\theta=23.4^{\circ}, \phi=0^{\circ}\right)$. It should be noted that the phase-matching angle was very different from the desired one, which was the same as in the laser in Salamanca: $\sim 31^{\circ}$ (for $\lambda_{\mathrm{p}}=397.5 \mathrm{~nm}$ ). The only reason for using this crystal was that it was the best one available at the laboratory at the time.

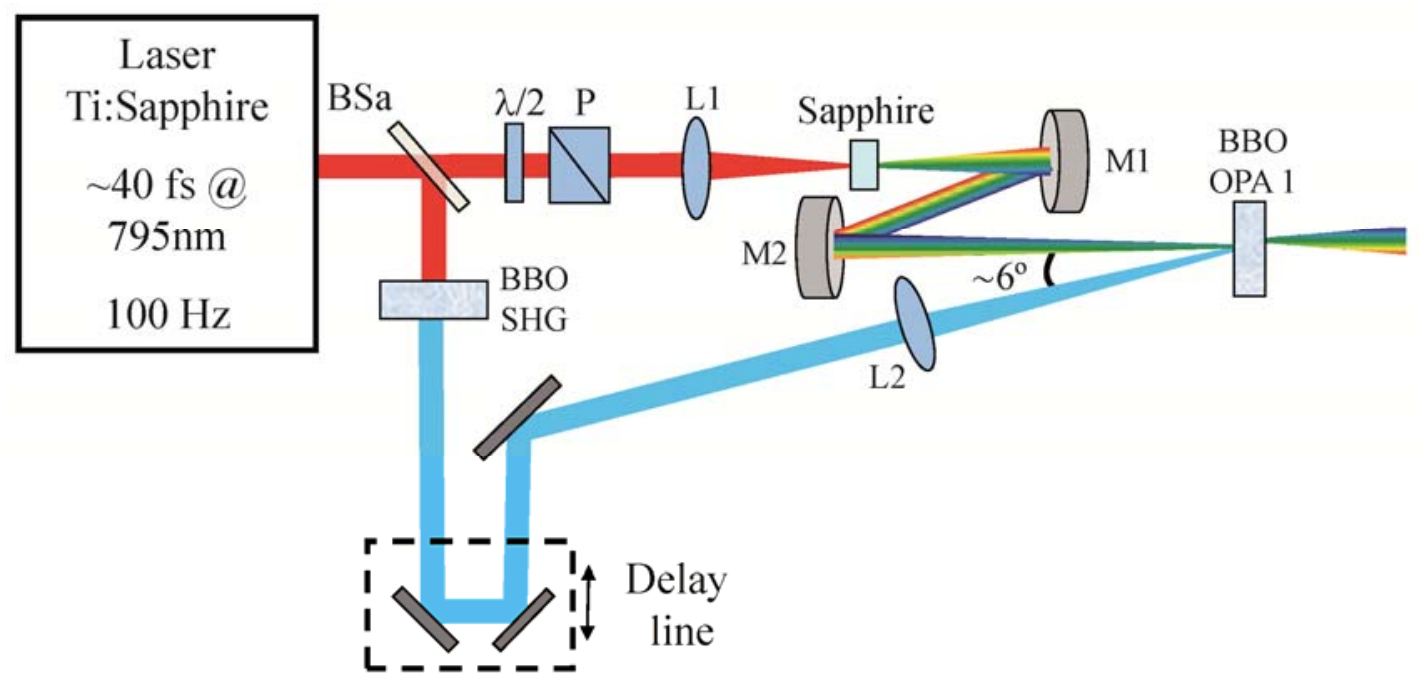

Figure 7.20. Sketch of the Vis-NOPA set-up that was developed in the CELIA laboratory. BSa: beam sampler, L1,2: lenses, M1,2: spherical mirrors, $P$ : polarizer. 
The main difference with the set-up in Salamanca (See Figure 7.10) was that in this case allreflective optics was used to guide the seed pulses. This greatly reduced the amount of chirp introduced into the $\mathrm{SC}$, which as previously discussed arises from the propagation of the broadband source in the refractive elements. As indicated in the figure, the SC was also generated in sapphire: in particular, we used a 3-mm-thick sample.

In the case of this NOPA it was difficult to make measurements of the energy of the pump beam, because it was not possible to separate the blue from the unconverted fundamental since the mirrors used to conduct the beam were merely mirrors with poor reflectivity in the IR region but not dichroic mirrors, as in the case in Salamanca. Moreover, no colour filter was available. Despite this, we estimated a blue energy of $21 \mu \mathrm{J}$. In this case, we also worked at a distance behind the focus of this lens.

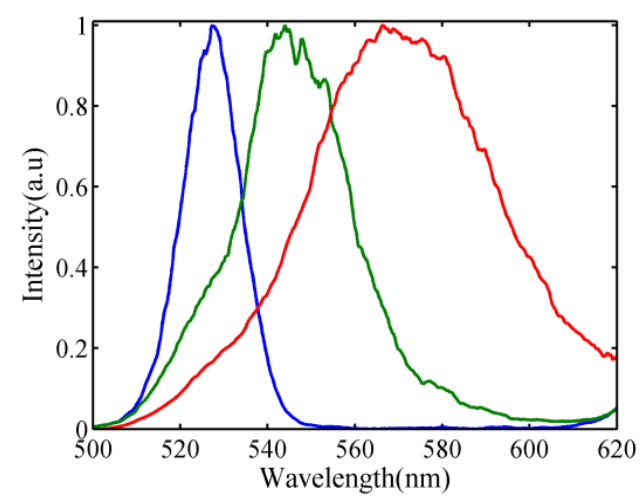

Figure 7.21. Spectra of amplified pulses measured after the NOPA amplification stage. Each spectrum was obtained changing the relative delay between the SC pulses (signal) and the blue ones (pump).

In the Figure 7.21 we see different amplified spectra, and in the table below the central wavelength, the intensity FWHM bandwidth, and the calculated Fourier-Transform limit. It can be seen how we reduced the Fourier limit in duration with respect to the measurements in Salamanca, (see Table 7.1), as expected from the broader amplified spectra obtained. The reason for this is that in this case the temporal duration of the pump and seed pulses were more similar, due to the use of all-reflective optics for focusing the SC.

It may be seen that how the spectra of the amplified pulses are narrower in the case of the lower wavelengths. We have already explained this issue in the case of OPA in Salamanca, although here the effect was more evident. However, in this case we were unable to make the same calculation as we did before because we did not have any measurements of the instantaneous frequency of the SC pulses.

\begin{tabular}{c|ccc}
\hline & Blue & Green & Red \\
\hline$\lambda_{\mathbf{c}}(\mathbf{n m})$ & 527 & 544 & 570 \\
$\Delta \lambda(\mathbf{n m})$ & 16 & 28 & 53 \\
FL (fs) & 30 & 18 & 10 \\
\hline
\end{tabular}

Table 7.3.Corresponding central wavelength, intensity FWHM spectral bandwidth, and temporal Fourier Limit (FL) of the pulses shown in Figure 7.21. 


\subsection{Future Directions}

Concerning future directions, in the case of OPA in Salamanca there are several improvements that can be made. The first would be to use reflective optics to focus the continuum; with this, the bandwidth of the amplified pulses would be larger, as was demonstrated in the NOPA in Bordeaux. The next logical step would be to compensate for the chirp introduced in the SC stage and attempt to generate shorter pulses. Also, optimization and characterization in terms of efficiency would be needed. In order to increase the gain factor it would be mandatory to improve the beam quality and match the sizes of both the SC and pump beams, as argued before.

Once we have gained more experience in working with OPA in the visible spectrum, it would be interesting to build an OPA in the near IR region, pumped directly with the $800 \mathrm{~nm}$ fundamental pulses. For this, however, new equipment would be needed, such as for example an IR spectrometer, which is not yet available at the laboratory. The IR-OPA will broaden the range of wavelengths accessible for application in other experiments even more.

\subsection{OPCPA: Extending OPAs to the Petawatt Level}

So far we have discussed how, with OPAs, we could obtain broadly tunable sources, although with moderate energy. However, some favourable properties of OPAs suggest that they could $[15,119]$ be applied in large-scale high-peak-power amplification systems. Currently, this possibility is known [17]. In fact, OPCPA has been proposed to replace conventional CPA-based systems for the generation of high peak-power and intensities. Now that we have introduced both the CPA and the OPA, we feel it a good idea to conclude this chapter with the following discussion.

In general, the term OPCPA, which stands for Optical Parametric Chirped Pulse Amplification, is used to describe the more energetic OPA schemes, which use stretching and compression stages, whereas OPA is reserved for the kind of system such as those described in this chapter. However, if we only look at the meaning of the acronym OPCPA, the OPA described here also involves the optical parametric amplification of a chirped signal. Another distinctive feature is that in OPA the signal and the pump are synchronized automatically, because they come from the same laser system, while in most OPCPA schemes they must be electronically synchronized, by an external system, because they are generated by two independent lasers. In the OPCPA scheme parametric gain is achieved by coupling a quasi-monochromatic high-energy pump field, such as a nanosecond pulse generated by a Nd:YAG laser, to a chirped, low-energy broadband seed field in a nonlinear crystal. If the seed pulse is sufficiently stretched, good energy extraction from the pump field can be achieved, and as in conventional CPA, subsequent recompression makes it possible to reach very high peak powers.

We can start the discussion by revising the problems or limitations in conventional CPA:

- The high-energy levels achieved are close to the damage threshold in the gratings of the compressor. Therefore to increase peak power the pulse duration must be reduced, which requires higher pulse bandwidths.

- The temporal contrast of the pulses is degraded by pre- and post-pulses arising through the amplification of spontaneous emission.

- Linear and nonlinear phases are accumulated in long paths, which prevents Fourier transform limited pulses from being generated and from focusing at the limit of diffraction. 
Before going into the details of the benefits of the OPCPA we shall explain the differences in the type of the amplification process itself.

In laser amplification, i.e. amplification in a gain medium such as Ti:Sapphire, the energy of the pump is stored in the material in terms of population inversion. In other words, the highly energetic pump is used to excite the electrons in a medium to an upper energy level with a long-term life. Later, the input seed pulse stimulates the decay of the electrons, with the consequent laser emission, and relaxation to the lower laser level.

In many laser amplifiers gain occurs over a four-level structure. Therefore the optical pumping is followed by non-radiative decay to the upper lasing lever. Then, the stimulated emission to the lower lasing level occurs and finally another non-radiative decay to the ground-state occurs. It is due to these non-radiative processes that a large amount of the pump energy is transferred to the gain medium itself, and consequently the medium heats, which can lead to a distortion in the phase and shape of the amplified pulses, and also limits the repetition rates.

Unlike OPA, we can consider the energy transfer as a photon being transiently absorbed by the medium, at the pump wavelength $\omega_{3}$. See Figure 2.10. This photon causes the atom to jump to a virtual excited state from where stimulated emission via the seed photon at $\omega_{1}$, causes the decay, first to a virtual state, and then to the ground state, with the emission of a pair of photons at $\omega_{1}$ and $\omega_{2}$ respectively. Recall that these so-called virtual states are not energy eigenlevels of the free atom, and that they only represent the combined energy of one of the energy eigenstates of the atom and one or more photons of the radiation field.

Moreover, a laser amplification medium can only amplify frequencies corresponding to the allowed transitions between the states of the medium. In crystals such as Ti:Sapphire the lasing transition occurs between the upper state and a large number of vibrational levels of the lower states, corresponding to a large spread of photon energies. However, the bandwidths available from this type of process are still exceeded by the available bandwidths in OPA amplifiers, in particular in the noncollinear interaction case, as we have seen in this chapter.

Nevertheless, on one level both processes are similar, since they both involve the absorption of a high-energy pump and the stimulated emission of a lower frequency photon in the presence of a second frequency field. However the OPA process is a parametric process, in which the atom ends up in the same ground state as it started. This means that the OPA process cannot transfer any energy to the crystal, and the heating of the amplifier medium due to pump absorption does not occur. One implication of this is that OPA amplifiers can in principle run at extremely high repetition rates.

Therefore, the benefits of OPCPA with respect to conventional CPA can be summarized as:

- High gain over ultrabroad bandwidths, mainly in noncollinear geometry, which provides gain bandwidths that are well in excess of those of conventional amplifiers. For example the gain bandwidth could sustain pulse spectra corresponding to a transform-limited duration of approximately 5 fs $[110,112]$.

- High energies are possible by using large nonlinear crystals, such as KDP which can be grown to sizes of tens of centimetres. These crystals should be capable of withstanding pump energies of hundreds of Joules [5]. 
- High-gain in a single pass, which on the one hand avoids the use of complicated multipasss set-ups and on the other reduces the number of passes inside the material and also reduces the extension of the amplifier.

- Low amplified spontaneous emission, which enhances the contrast ratio [144].

- An Almost complete absence of thermal loading, in the amplification process, for the reasons commented above. This eliminates spatial aberration effects on the beams and diminishes the linear and nonlinear phase distortions.

However CPA technology has been subject to more testing over more than 20 years of operation, while OPCPA is just beginning to be assayed. As expected, OPCPA also has its limitations, which can be summarized as: thermal loading due to residual absorption of the pump from the nonlinear crystals; possible optical damage in the same, and high alignment sensitivity and low pulse-to-pulse stability. The latter two are related to the fact that the seed and pump pulses must be perfectly overlapped in time for and space for amplification to occurs efficiently.

As commented in Chapter 1, one possible option for PW peak power lasers are hybrid systems [18], in which the initial amplification stages (front-end) are based on OPCPA technique and the power amplifiers are conventional CPA. 



\section{Chapter 8}

\section{Materials Micro-Processing with Vis- UV Femtosecond Pulses: Applications}

\subsection{Introduction. Ultrafast Ablation}

Materials micro-processing with femtosecond pulses has attracted huge interest since the development of the CPA technique. In recent years, the scope of practical applications where the suitability of these ultrashort laser sources has been demonstrated has become increasingly important. This is mainly due to the special properties of the laser-matter interaction with intense femtosecond pulses.

Above $10^{10} \mathrm{~W} / \mathrm{cm}^{2}$, the standard Einstein's photoelectric effect gives rise to multiphoton effects. Multiphoton ionization -the ionization of atoms and molecules by the simultaneous absorption of several photons - is the dominant effect in laser-matter interactions up to $10^{14}-10^{15} \mathrm{~W} / \mathrm{cm}^{2}$. Beyond these intensities, other ionization mechanisms as tunneling or barrier suppression become more relevant. In the particular case of dielectrics or semiconductors, the multiphoton ionization rate depends on the intensity as $I^{m}, m$ being the minimum number of photons needed to overcome the bandgap of the material. This strong dependence of ionization on the laser intensity, highly nonlinear, allows very localized damage to the target once the pulses are tightly focused on it. Such nonlinearity allows structuring even below the diffraction limit, thus allowing ultra-high precision applications where the use of other lasers is inadvisable.

Additionally, during the interaction of the pulse with the solid target, the ultrashort temporal duration of femtosecond pulses prevents the direct coupling of the electromagnetic radiation with 
the ionic lattice. Therefore, thermal effects are minimized with this kind of interaction resulting in very clean boundaries between the processed and unprocessed parts of the sample.

When the laser pulse is focused on the surface of a solid target, the ionization leads to a modification of the physical and/or chemical properties of the matter. If the ionization is strong enough, ultrafast ablation, a very complex mechanism, takes place. This leads to the removal of material in localized areas, where the laser fluence exceeds some threshold (dependent on the target but typically in the range of a few $\mathrm{J} / \mathrm{cm}^{2}$ ). These are the fundamentals of micro-crater or microchannel fabrication.

One of the most impressive capabilities of infrared femtosecond lasers is the possibility of performing the three-dimensional processing of transparent dielectrics. Since the central wavelength of Ti:Sapphire-based femtosecond laser systems (around $800 \mathrm{~nm}$ ) lies in the transparency range of most crystals and optical glasses, the pulses can be focused at any point inside the dielectric, leading to the localized modification of certain physical properties of the material. By accurately setting the irradiation conditions, the nature and size of the modifications induced can be controlled to some extent. This property has been applied to high-precision microfabrication of a number of optical devices: optical waveguides, photonic crystals, three-dimensional optical storage systems, or diffractive elements embedded in glasses or crystals, among many others.

\subsection{Effect of Wavelength in Femtosecond Micro-Processing}

In laser-matter interactions with long laser pulses (nanosecond), several "linear" effects cause a different behaviour of the material with the wavelength. In particular, the linear absorption of the material plays a fundamental role in governing the dynamics of such interactions, and owing to the dispersive properties of all materials the absorption/transparency of the material will change with wavelength.

In the case of the interaction with ultrashort laser pulses, in addition to linear effects nonlinear processes must also be taken into account. In fact, processes such as ultrafast ablation are mainly governed by nonlinear effects. The dependence of the ablation threshold on different laser parameters has been investigated in depth in the literature, and in particular, the role of the laser wavelength on ultrafast laser ablation has been shown to be important. As stated before, the main mechanism leading to electron plasma formation is multiphoton ionization. In all multiphoton processes, the transition probability strongly decreases as the number of absorbed photons required to reach the upper level increases: in other words, if the multiphoton order is very high, transitions through many virtual states are needed, and the final transition probability drops dramatically. Evidently, if the laser wavelength that drives the process is decreased, the multiphoton order will also decrease; the number of intermediate virtual states decreases and thus, in general, the transition probability will increase. As a consequence, it is expected that the ablation or damage thresholds will decrease as the wavelength decreases because less fluence is required to produce the plasma.

This effect has been investigated in a few works. In [145], both the effect of wavelength and the pulse duration on the damage of fused silica and calcium fluoride were studied by comparing the fundamental and the second harmonic of a Ti:Sapphire femtosecond laser. For all the pulse durations, a factor of more than 2 was found between the damage threshold for the fundamental (larger) and the second harmonic (smaller). A more exhaustive study of this dependence is reported 
in [138]. In that work, the authors used an OPA that allowed them to continuously tune the laser wavelength from 250 to $2000 \mathrm{~nm}$. The results concerning the damage to the same materials (fused silica and calcium fluoride) revealed a continuous increase in the damage threshold with the wavelength from the UV to a wavelength around $800 \mathrm{~nm}$. Above this wavelength the threshold remained nearly constant within the error bars of the experiment. Similar results have been found for other dielectric materials and even biological tissues such as like corneal stroma [146].

\subsection{Applications}

In this section we shall briefly present some of the most interesting experiments that have been carried out at the laboratory of Micro-procesado de materiales con láser at the Universidad de Salamanca concerning the micro-processing of materials with femtosecond pulses in the visible or UV wavelength range. The beams used for these experiments were all designed and characterized within the framework of this thesis.

\subsubsection{Silicon Nano/Micro-Structuring}

The surface structuring of silicon with femtosecond pulses [147] has attracted much attention due to the many different fields where this material is essential. The generation of a controlled patterning on the surface is very interesting because it allows the physical properties of the bare silicon to be changed. For example, it has found applications ranging from studies on hydrophobicity [148] to the development of alternative energies [149].

Another research field where nano/micro-structured silicon has been seen to be beneficial is the MALDI-TOF (Matrix Assisted Laser Desorption/Ionization-Time Of Flight) mass spectrometry. This spectroscopic technique is one of the most common tools for the analysis of biological samples, and in particular for the detection of low concentrations of drugs in these samples. However, it is necessary to use a matrix in the desorption/ionization process, which frequently masks the peaks corresponding to the drug to be measured. Several techniques aimed at eliminating the use of a matrix have been developed, among which the employment of ablated silicon wafers is one of the most promising ones [150] since this allows a virtually background-free desorption in the low mass region.

In collaboration with the Departamento de Química-Física (Universidad del País Vasco), we have investigated different strategies to produce femtosecond-structured silicon samples for their application in the detection of drugs with MALDI-TOF spectroscopy. Among the different experimental set-ups found in literature, we adopted that described in [151]: in sum, this consists of placing the sample in a glass container under a water column of a given thickness, and irradiating the sample with the second harmonic of the Ti:Sapphire laser. To this end, we used the SHG system described in Chapter 3. The main parameters to be controlled are the pulse energy, the scanning velocity and the focusing conditions. A systematic study of the morphologies produced with many irradiation conditions was performed with AFM and SEM. Finally, certain parameters were selected with which it was possible to produce nano-structures, with a very large degree of homogeneity along the irradiated surface. In Figure 8.1 a) we show some results of the samples processed.

The main mechanism that produces the structuring in the silicon samples is local field enhancement at the surface. When the laser pulse impinges on the surface at high-fluence, some gas 
bubbles are produced at the silicon-water interface due to the increase in temperature. If the bubble survives for a time longer than $1 \mathrm{~ms}$ (mean time between two pulses at $1 \mathrm{kHz}$ ), the subsequent incident pulse will be scattered by the bubble (or bubbles), leading to a inhomogeneous local field distribution at the surface: diffraction and refraction will modify the incident energy distribution. In Figure $8.1 \mathrm{a}$ ), this effect can be seen clearly as the circular ring patterns that appear. The cumulative effect of many incident pulses interacting with many bubbles creates the initial surface patterning that will eventually produce the surface structuring. The dependence of the structuring on the pulse wavelength is clear since the local field distribution, which is an undulatory effect, strongly depends on wavelength: the shorter the wavelength, the smaller the surface structuring.

Silicon samples structured with both wavelengths (795 and $398 \mathrm{~nm}$ ) were tested for MALDITOF experiments, and the best results were obtained with the smallest surface structures, corresponding to the shortest wavelength. This technique offers as a very promising tool for improving MALDI-TOF detection capabilities.
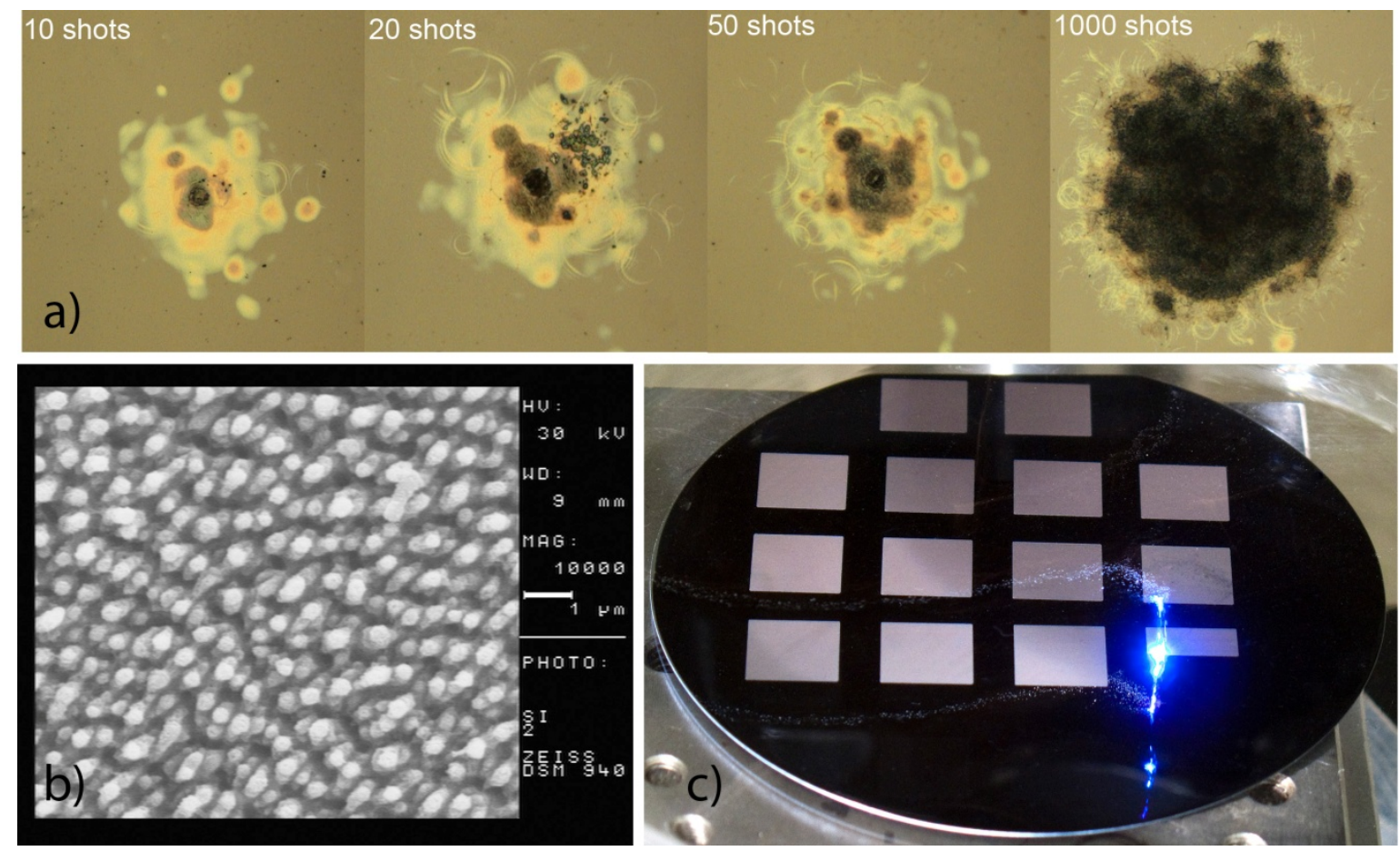

Figure 8.1. a) Evolution of the surface of a silicon sample with the number of incident laser pulses (as indicated in each frame). b) SEM image of the surface after the surface processing. Spikes of around $300 \mathrm{~nm}$ width can be seen. c) Picture of the silicon sample during irradiation.

\subsubsection{Selective Elimination of Varnish Layers in Tempera Paints}

The laser cleaning of paints and works of art has been addressed in of many studies, in which the possibilities and limitations of the technique are discussed. The laser systems that have been most frequently used for this purpose are typically pulsed lasers in the nanosecond regime. For this pulse duration, one of the most relevant parameters to ensure a successful (and harmless) cleaning is the laser wavelength, since this determines the efficient or inefficient coupling of the laser energy to the unwanted layer. If the laser wavelength falls within the high absorbance spectral region of the material to be removed, this coupling is ensured. For instance, the removal of polymerized outer 
varnish layers and over-paintings has been demonstrated with a $\mathrm{KrF}$ excimer laser operating at 248 $\mathrm{nm}$ with a nanosecond pulse duration [152]. More recently, some studies have been conducted using picosecond or femtosecond laser pulses for the same purpose. The prospects of ultrashort pulses for the removal by laser of unwanted superficial layers and overpaints relies on the minimization of photo-thermal, photo-mechanical, and photo-chemical phenomena, on the possibility of processing even nominally transparent substrates, and on optimizing morphological aspects to avoid melting, bubbling, crack formation, etc.
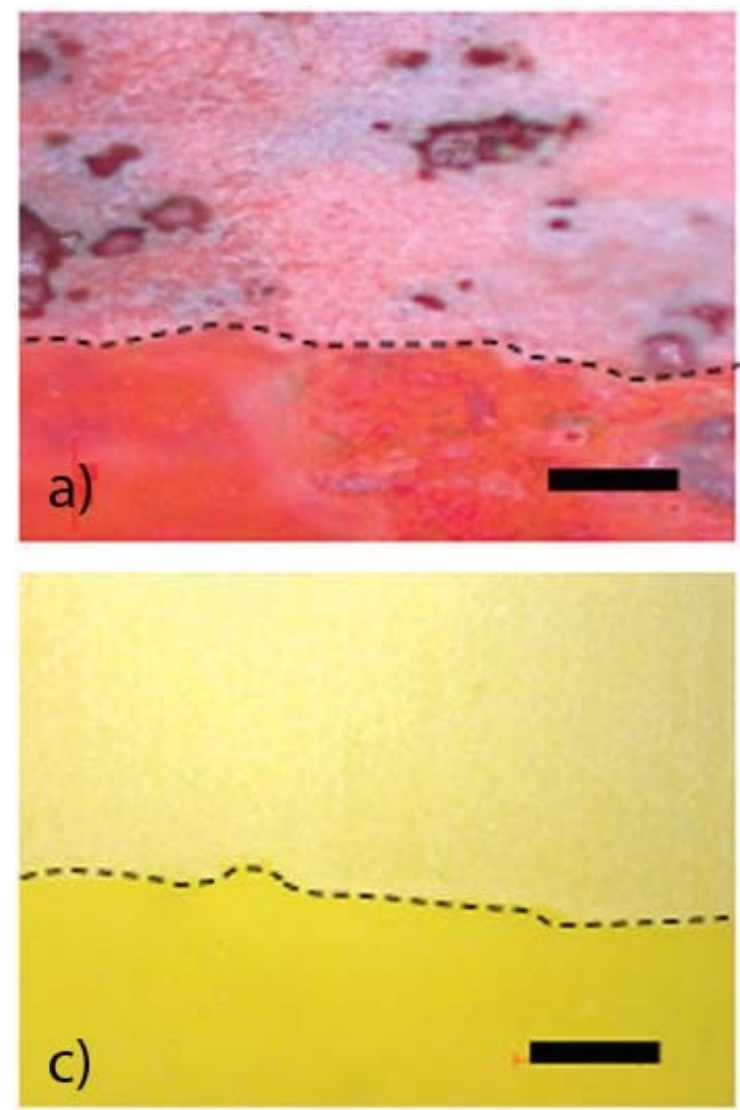
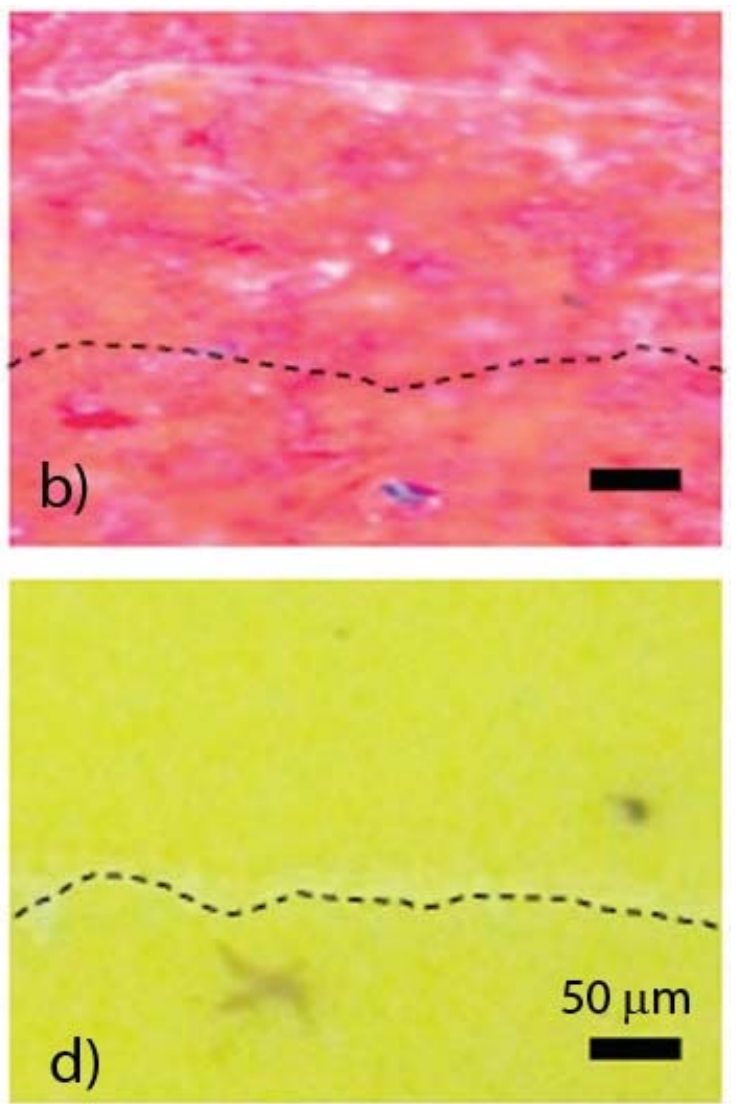

Figure 8.2. Optical microscopy pictures of two different samples (vermillion and lead chromate) irradiated with $265 \mathrm{~nm}$ femtosecond pulses (left) and $213 \mathrm{~nm}$ nanosecond pulses (right). The dashed line represents the boundary between the processed and unprocessed areas.

In principle, for laser cleaning with femtosecond pulses it could be expected that the laser wavelength would not be a parameter as relevant as it is when nanosecond pulses are used, because the fundamentals of ultrafast laser ablation rely on highly nonlinear processes. In collaboration with researchers at the Instituto de Química Física Rocasolano (Consejo Superior de Investigaciones Cientificas - CSIC), we performed a systematic study of the capabilities of femtosecond lasers for the selective removal of varnish layers, by comparing the results obtained with those obtained with nanosecond pulses [67]. The samples consisted of single layers of tempera paints covered by a $\sim 10$ $\mu \mathrm{m}$-thick shellac varnish and they were irradiated with the fundamental $(798 \mathrm{~nm})$ and the second $(398 \mathrm{~nm})$ and third $(265 \mathrm{~nm})$ harmonics of the femtosecond laser system. A preliminary study conducted to determine the ablation threshold of the varnish for the three wavelengths. Then, the samples were irradiated at fluences slightly smaller and greater than the threshold by scanning the 
surface with the beam focused loosely. The irradiated samples were analyzed by several techniques (optical microscopy, colorimetry and laser-induced fluorescence) in order to elucidate the magnitude of the modification induced in the substrate (the tempera paint) by the laser irradiation.

Poor results were obtained with all three wavelengths. The varnish layer either ablated at fluencies that exceeded the damage threshold of the underlying paint (at $398 \mathrm{~nm}$ ) or underwent irreversible modifications to the initially smooth surface texture in the form of a laser-generated foam that reduced its transparency (at $265 \mathrm{~nm}$ ). Moreover, the underlying paint layers underwent colour changes to different extents. These effects were not so dramatic when using $213 \mathrm{~nm}$ pulses in the nanosecond regime. This wavelength lies close to the absorption peak of shellac varnish while $265 \mathrm{~nm}$ and $398 \mathrm{~nm}$ are at the tail or distant from the peak respectively. This suggests that linear absorption is crucial in this process. Further investigations into the use of shorter wavelengths with femtosecond pulses would be really interesting since this would cast light on the different mechanisms involved in the different temporal regimes. It could be done by using the fourth harmonic of the Ti:Sapphire laser $(199 \mathrm{~nm})$, and indeed we hope to be able to do this in the near future.

\subsubsection{Fabrication of Optical Waveguides in Active Crystals}

Femtosecond laser inscription has been shown to be a very powerful technique for the fabrication of waveguides in crystals and glasses with controlled properties via adequate choice of the experimental parameters. Typically, the laser is focused by a microscope objective at some distance beneath the surface of the sample while it is moved. The high-intensity achieved at the focus of the lens creates a plasma, which eventually modifies the properties of the material. If the irradiation parameters (mainly the pulse energy and scanning speed) are suitably chosen, some increase in the index of refraction is produced at the focal region, leading to a waveguide along the direction scanned [153].

a)

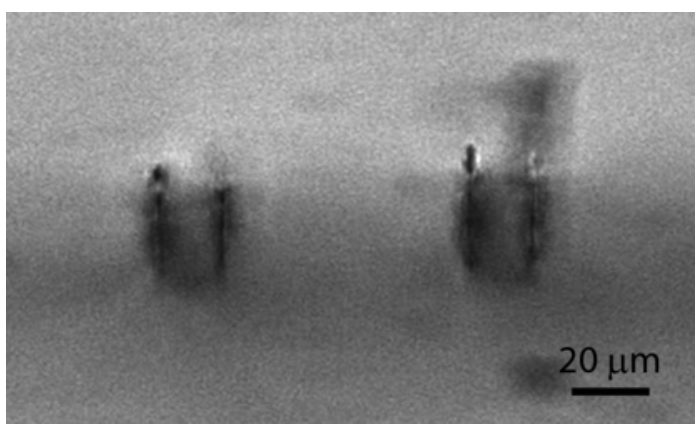

b)

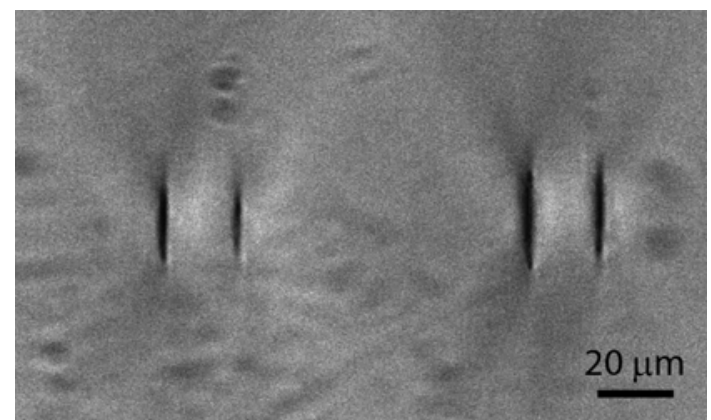

Figure 8.3. Waveguides written in a ceramic Nd:YAG sample by the double scan technique by using: a) infrared femtosecond pulses $(795 \mathrm{~nm}, 120 \mathrm{fs})$, b) visible femtosecond pulses $(397.5 \mathrm{~nm}, 150 \mathrm{fs})$, both sets with analogous irradiation conditions (scanning velocity, focusing optics and pulse energy).

A different approach is the so-called "double scan" technique, which consists of performing two scans in the sample some microns distant one from each other, using large pulse energies that produce severe damage to the material. In this case, with the correct choice of parameters, an optical waveguide appears between the two tracks produced by the laser. This technique is much more interesting than the single-scan method for manufacturing active photonic waveguides (i.e. 
laser waveguides), because in this case the spectral properties of the active ion are very well preserved, as has been demonstrated in different crystals [154-157]. Moreover, the distance between adjacent tracks is another parameter that facilitates the control of the guided-mode properties.

In most studies, the wavelength used in the manufacturing procedure is around $800 \mathrm{~nm}$ : the central wavelength of the Ti:Sapphire laser systems. However, much attention has been paid to the role of the laser wavelength in the inscription procedure. In collaboration with researchers from the Grupo de Espectroscopía Láser (Universidad Autónoma de Madrid) we have performed preliminary studies on waveguide manufacture in Nd:YAG with $398 \mathrm{~nm}$ femtosecond laser pulses. Although more detailed analyses are needed, visual inspection of the waveguides manufactured by optical microscopy reveals some differences between the UV manufactured waveguides and standard IR (see Figure 8.3). On the one hand, when working with UV some residual damage to the material is observed above and below the main damage tracks. This can be attributed to the lower multiphoton order for ionization. On the other hand, the main damage tracks seem to be very thin and well defined: this would be very interesting for guiding properties because it could reduce losses. Further work addresing the characterization of the waveguides is needed, but results obtained so far are very promising. 



\section{Conclusions}

In summary, this work has been focused on the development and implementation of different strategies to access the visible and near ultraviolet spectral ranges with femtosecond laser pulses through nonlinear optical processes in crystals. We have used both standard techniques as novel schemes that improve some properties of the generated pulses.

\section{Second and Third Harmonic Generation}

- In the first part, we have designed, built and characterized a module to generate the second $(398 \mathrm{~nm})$ and third $(265 \mathrm{~nm})$ harmonics of the Ti:Sapphire laser, preserving the spectral and temporal properties of the fundamental. Although it was specially adapted to the source available in the laboratory, it could be easily modified to frequency doubling or tripling others beams if it were necessary. Two nonlinear crystals (BBO) of different thickness were characterized in terms of conversion efficiency, in the second and third harmonic generation processes, suggesting that both crystals were suitable for the different applications.

- The study was completed with the temporal characterization of the generated second harmonic pulses, which could be performed in the module itself. This fact is a distinctive of our system with respect to the commercial ones and makes it very interesting, since in some applications a control on the pulses time duration is required. These two new laser wavelengths are nowadays available in the laboratory of Micro-procesado de materiales con láser and have been extensively used in many practical applications.

\section{Noncolinear Sum-Frequency Generation}

- A novel scheme for SFG in a noncolinear configuration was designed and implemented. A BBO crystal was micro-structured by ultrafast laser ablation, thus recording a 1D diffraction grating on the surface. The micro-structured crystal was used to induce the noncollinear propagation of fundamental and second harmonic femtosecond pulses, achieving phase matching between certain propagation directions thus generating efficiently the third harmonic. 
- In a certain configuration, the GVM between the incident pulses (fundamental and second harmonic) can be effectively eliminated, demonstrating the possible application of this device in pulse characterization measurements of femtosecond pulses. Consequently, our approach permits to alleviate the constrain of using very thin crystals (usually more expensive and less efficient) when performing this kind of measurements, in fact, the crystal needed to perform them should ideally be 10 times thinner than the one in which the grating was recorded.

- The technique could be of interest in other nonlinear processes like noncollinear optical parametric amplification, or in different crystals like periodically poled materials or in random quadratic media.

\section{Diffractive Lenses and Optical Nonlinear Processes}

- In a first set of experiments, a kinoform DL was used to focus a femtosecond beam in a BBO crystal in order to generate the second and subsequently the third harmonic. With this element it is possible to achieve tunability in the generated beams by simply scanning the nonlinear crystal around the focus of the lens.

- The effect is well explained in terms of chromatic aberration. The generated pulses were characterized, thus demonstrating that they maintain their duration in the femtosecond regime, and their spectra is kept compatible with the clipped spectrum that arises due to the strong chromatic aberration of the lens.

- In a second set of experiments, the same diffractive lens was applied to generate SC pulses in sapphire or fused silica bulk samples. The process of SC generation has revealed new and interesting features, which are not present when using achromatic lenses. On the one hand the SC has absence of red wavelengths and a prominent peak appears in the blue; on the other, the peak can be tuned in the blue part of the spectrum, over $50 \mathrm{~nm}$, simply by changing the lens-sample distance. The influence of the numerical aperture, input energy, and the wavelength stability has been studied in detail. The temporal characterization of such tunable pulses in the blue is now in progress.

- In summary, the use of diffractive lenses for triggering nonlinear optical processes is of great interest due to their potential to tailor the spectral features of the generated pulses. The potentials in other interesting parametric processes, OPA for instance, is now under consideration.

\section{Noncolinear Optical Parametric Amplification}

- The last system developed to change the range of emission of the Ti:Sapphire laser was a broad-band NOPA in the visible part of the spectrum, with a tunability from 450 to $750 \mathrm{~nm}$. The setup consisted on two amplification stages (BBO crystals) employing as seed pulses the SC generated in fused silica or sapphire. This was indeed the first tunable source available in the laboratory at Salamanca. The setup was used to characterize the SC pulses generated in bulk so that the effect of dispersion in the optical elements was analyzed. 
- An alternative NOPA system with all-reflective optics was investigated in collaboration with the group of Lasers Femtosecondes, Développements Optiques in Bourdeaux. The differences and advantages between both schemes are discussed.

- Nowadays our efforts are deposited on developing a compact all-reflective system and optimizing the setup to maximize the parametric gain. 



\section{Appendix A}

\section{Definition of Nonlinear Susceptibility}

In Chapter 2 we considered the nonlinear interactions that can occur in a medium that was lossless and dispersionless. In such a case, the nonlinear polarization could be described by the power series of the electric-field strength Eq. (2.2). Therefore, to introduce the nonlinear susceptibility properly we shall consider a more general case of a material with dispersion and loss. Within this new context, nonlinear susceptibility becomes a complex tensor relating the complex amplitudes of the electric field and polarization vectors.

First, we write the total electric field:

$$
\tilde{\mathbf{E}}(\mathbf{r}, t)=\sum_{n} \mathbf{A}\left(\omega_{n}\right) e^{i\left(\mathbf{k}_{n} \cdot \mathbf{r}-\omega_{n} t\right)}
$$

with a summation over the positive and negative frequency components, where $\mathrm{A}\left(\omega_{n}\right)$ are the slowly varying amplitudes. Similarly, the nonlinear polarization can be written:

$$
\tilde{\mathbf{P}}(\mathbf{r}, t)=\sum_{n} \mathbf{P}\left(\omega_{n}\right) e^{-i \omega_{n} t}
$$

As we have mentioned in this more general case the quantity that relates the polarization to the applied electric field is a tensor, not a scalar quantity as we assumed in Eq. (2.2). However we shall not include a complete description of the nonlinear susceptibility, simply an introduction that will serve us to illustrate its properties.

We shall introduce the amplitude of the polarization in a medium with second order susceptibility: 


$$
P_{i}\left(\omega_{n}+\omega_{m}\right)=\varepsilon_{0} \sum_{j k} \sum_{(n m)} \chi_{i j k}^{(2)}\left(\omega_{n}+\omega_{m}, \omega_{n}, \omega_{m}\right) E_{j}\left(\omega_{n}\right) E_{k}\left(\omega_{m}\right)
$$

Here, $P_{i}\left(\omega_{n}+\omega_{m}\right)$ describes the polarization associated with the sum frequency generation process, and the indices $i, j, k$ refer to the Cartesian components of the electric field. The notation $(n, m)$ indicates that in performing the summation over $n$ and $m$, the sum $\left(\omega_{n}+\omega_{m}\right)$ is to be held fixed, although $\omega_{n}$ and $\omega_{m}$ are allowed to vary. The amplitude $E\left(\omega_{n}\right)$ is associated with the $\exp \left(-i \omega_{n} t\right)$, time dependence and the amplitude $E\left(\omega_{m}\right)$ is associated with the $\exp \left(-i \omega_{m} t\right)$ time dependence, their product $E\left(\omega_{n}\right) E\left(\omega_{m}\right)$ is associated with the $\exp \left(-i\left(\omega_{n}+\omega_{m}\right) t\right)$ time dependence.

To illustrate this, let us consider the mutual interaction of three waves with frequencies of $\omega_{1}$, $\omega_{2}$, and $\omega_{3}=\omega_{1}+\omega_{2}$. The complete description of their interaction requires that we must know the nonlinear polarizations influencing each of them. Therefore we need to determine the six tensors:

$$
\begin{array}{lll}
\chi_{i j k}^{(2)}\left(\omega_{1}, \omega_{3},-\omega_{2}\right) & \chi_{i j k}^{(2)}\left(\omega_{2}, \omega_{3},-\omega_{1}\right) & \chi_{i j k}^{(2)}\left(\omega_{3}, \omega_{1}, \omega_{2}\right) \\
\chi_{i j k}^{(2)}\left(\omega_{1},-\omega_{2}, \omega_{3}\right) & \chi_{i j k}^{(2)}\left(\omega_{2},-\omega_{1}, \omega_{3}\right) & \chi_{i j k}^{(2)}\left(\omega_{3}, \omega_{2}, \omega_{1}\right)
\end{array}
$$

and their negatives. Since each of these tensors thus consists of $3^{3}=27$ Cartesian components, as many as $12 \times 27=324$ different components (complex numbers) need to be specified in order to describe the interaction. At this point, the need to determine this large amount of numbers may seem a rather arduous task, although, the nonlinear susceptibility and the crystals have some symmetry properties that considerably reduce the values that must be addressed, and hence usually far fewer than 324 numbers are necessary to describe the nonlinear coupling.

The first symmetry deals with the reality of the fields. Addressing this issue, we may conclude that the positive and negative frequency components of the susceptibility are related as:

$$
\chi_{i j k}^{(2)}\left(-\omega_{n}-\omega_{m},-\omega_{n},-\omega_{m}\right)=\chi_{i j k}^{(2)}\left(\omega_{n}+\omega_{m}, \omega_{n}, \omega_{m}\right)^{*}
$$

Then, we can apply the intrinsic permutation symmetry. Physically this condition is simply a statement that it does not matter which the first field is and which the second field is in products such as $E_{j}\left(\omega_{n}\right) E_{k}\left(\omega_{m}\right)$. Therefore, we have:

$$
\chi_{i j k}^{(2)}\left(\omega_{n}+\omega_{m}, \omega_{n}, \omega_{m}\right)=\chi_{i k j}^{(2)}\left(\omega_{n}+\omega_{m}, \omega_{m}, \omega_{n}\right)
$$

Finally, we have Kleinman symmetry. This symmetry requires that the optical frequencies be much lower than the resonance frequency of the material. This is indeed true for most nonlinear crystals, and in this case the material is necessarily lossless and the susceptibilities are effectively independent of the frequency. The requirement of intrinsic permutation symmetry mentioned above is that the interchange of the indices $j$ and $k$ in the nonlinear susceptibility will not change the value of this latter as long as the frequencies $m$ and $n$ are also interchanged. This enables us to write Kleinman`s condition as:

$$
\begin{aligned}
& \chi_{i j k}^{(2)}\left(\omega_{3}=\omega_{1}+\omega_{2}\right)=\chi_{j k i}{ }^{(2)}\left(\omega_{3}=\omega_{1}+\omega_{2}\right)=\chi_{k i j}{ }^{(2)}\left(\omega_{3}=\omega_{1}+\omega_{2}\right)= \\
& \chi_{i k j}{ }^{(2)}\left(\omega_{3}=\omega_{1}+\omega_{2}\right)=\chi_{j i k}{ }^{(2)}\left(\omega_{3}=\omega_{1}+\omega_{2}\right)=\chi_{k j i}{ }^{(2)}\left(\omega_{3}=\omega_{1}+\omega_{2}\right)
\end{aligned}
$$




\section{A.1. The Contracted Notation}

We now introduce the notation that is often used when the Kleinman symmetry condition is valid. We introduce the tensor

$$
d_{i j k}=\frac{1}{2} \chi_{i j k}^{(2)}
$$

and for simplicity we suppress the frequency arguments. The factor $1 / 2$ is a consequence of historical convention.

We now assume that $d_{i j k}$ is symmetric in its last two indices. This assumption is valid whenever the Kleinman symmetry condition is valid and in addition is valid in general for SHG, since in that case $\omega_{n}$ and $\omega_{m}$ are equal. Therefore, we can simplify the notation even more by introducing a contracted matrix $d_{i l}$ according to the prescription

$$
\begin{array}{ccccccc}
j k: & 11 & 22 & 33 & 23,32 & 31,13 & 12,21 \\
l: & 1 & 2 & 3 & 4 & 5 & 6
\end{array}
$$

Thus, the nonlinear susceptibility tensor can be represented as a $3 \times 6$ matrix

$$
d_{i l}=\left[\begin{array}{llllll}
d_{11} & d_{12} & d_{13} & d_{14} & d_{15} & d_{16} \\
d_{21} & d_{22} & d_{23} & d_{24} & d_{25} & d_{26} \\
d_{31} & d_{32} & d_{33} & d_{34} & d_{35} & d_{36}
\end{array}\right]
$$

And for example we can describe the nonlinear polarization leading to the generation of the second harmonic in terms of this matrix:

$$
\left[\begin{array}{c}
P_{x}(2 \omega) \\
P_{y}(2 \omega) \\
P_{z}(2 \omega)
\end{array}\right]=2 \varepsilon_{0}\left[\begin{array}{llllll}
d_{11} & d_{12} & d_{13} & d_{14} & d_{15} & d_{16} \\
d_{21} & d_{22} & d_{23} & d_{24} & d_{25} & d_{26} \\
d_{31} & d_{32} & d_{33} & d_{34} & d_{35} & d_{36}
\end{array}\right]\left[\begin{array}{c}
E_{x}(\omega)^{2} \\
E_{y}(\omega)^{2} \\
E_{z}(\omega)^{2} \\
2 E_{y}(\omega) E_{z}(\omega) \\
2 E_{x}(\omega) E_{z}(\omega) \\
2 E_{x}(\omega) E_{y}(\omega)
\end{array}\right]
$$

If we now explicitly introduce the Kleinman symmetry condition -that is, the three indices $d_{i j k}$ can be permuted freely-, we find that not all of the 18 elements of $d_{i l}$ are independent. For instance, note that:

$$
d_{12} \equiv d_{122}=d_{212}=d_{16}
$$

By applying this type of argument systematically we find that $d_{i l}$ has only 10 independent elements: 


$$
d_{i l}=\left[\begin{array}{llllll}
d_{11} & d_{12} & d_{13} & d_{14} & d_{15} & d_{16} \\
d_{16} & d_{22} & d_{23} & d_{24} & d_{14} & d_{12} \\
d_{15} & d_{24} & d_{33} & d_{23} & d_{13} & d_{14}
\end{array}\right]
$$

This matrix is even more simplified when taking into account the symmetries of the crystals. This can be consulted in textbooks such as [25].

\section{A.2. Effective Value of $d: d_{\text {eff }}$}

For a fixed geometry, i.e. for a fixed propagation and polarization direction, it is possible to express the nonlinear polarization giving rise to SFG and SHG by means of the scalar relationships

$$
\begin{gathered}
P\left(\omega_{3}\right)=4 \varepsilon_{0} d_{e f f} E\left(\omega_{1}\right) E\left(\omega_{3}\right) \\
P(2 \omega)=2 \varepsilon_{0} d_{e f f} E(\omega)^{2}
\end{gathered}
$$

where

$$
E(\omega)=|\mathbf{E}(\omega)| \quad \text { and } \quad \mathrm{P}(\omega)=|\mathbf{P}(\omega)|
$$

In each case, $d_{\text {eff }}$ is obtained by first determining $\mathbf{P}$ explicitly through the use of the matrix equation (A.11) and a similar one that can be obtained for the SFG. To illustrate how $d_{\text {eff }}$ is obtained, we shall use the BBO, a uniaxial crystal of the $3 \mathrm{~m}$ group. In this case, the susceptibility tensor is as follows:

$$
\left[\begin{array}{cccccc}
0 & 0 & 0 & 0 & d_{31} & -d_{22} \\
-d_{22} & d_{22} & 0 & d_{31} & 0 & 0 \\
d_{31} & d_{31} & d_{33} & 0 & 0 & 0
\end{array}\right]
$$

where $d_{22}=2.2 \mathrm{pm} / \mathrm{V}$ and $d_{13}=0.08 \mathrm{pm} / \mathrm{V}$ [39]. Note here the simplification as compared with the complete matrix in (A.11).

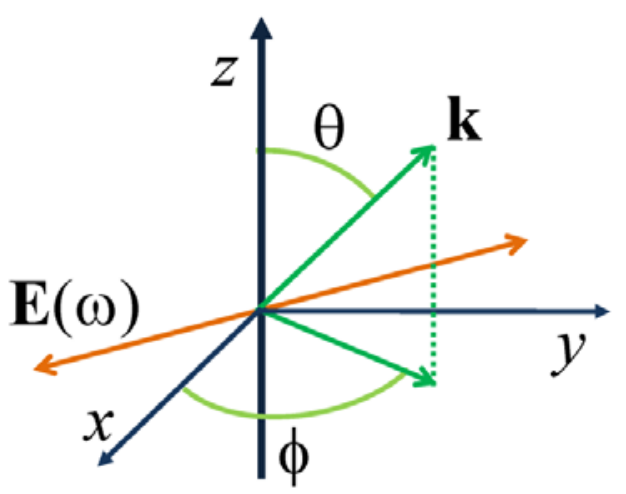

Figure A.1. Angles definition in a nonlinear crystal.

First we apply the Type I conditions. This means that the incoming field $\mathbf{E}(\omega)$ is polarized perpendicularly to the principal plane (see Sect. 2.7) containing the optical axis, $z$, and the 
propagation direction, $\mathbf{k}$. Thus $E_{z}(\omega)=0$. In Figure A. 1 we show the definition of the angles inside the crystal, where $\theta$ is the angle that the propagation vector $\mathbf{k}$ forms with the optical axis and $\phi$ is the azimuth.

The next step is to consider that the polarization at frequency $2 \omega$, is an extraordinary beam and, assuming that the walk-off angle is small, we have:

$$
d_{\text {eff }}=d_{31} \sin \theta-d_{22} \cos \theta \sin 3 \phi
$$

Similarly in the case of Type II phase matching, one obtains:

$$
d_{\text {eff }}=d_{22} \cos ^{2} \theta \cos 3 \phi
$$

The values for the tensor elements and equations for $d_{\text {eff }}$ describing interactions in other types of crystals can be found in [158] for almost all currently used nonlinear crystals.

If the walk-off angle $(\rho)$ is included, we obtain:

$$
\begin{gathered}
d_{e f f}=d_{31} \sin (\theta+\rho)-d_{22} \cos (\theta+\rho) \sin 3 \phi \\
d_{e f f}=d_{22} \cos ^{2}(\theta+\rho) \cos 3 \phi
\end{gathered}
$$





\section{Appendix B}

\section{Filter Characterization}

In this appendix we offer the experimental characterization of the two chromatic filters used in the experiments, FGB37s and FESO750, both from Thorlabs ${ }^{\circledR}$.

\section{The FGB37s Bandpass Filter:}

This filter is a coloured bandpass transmittance filter; it corresponds to the BG40 glass from SCHOTT ${ }^{\circledR}$. The filter's transmittance data are available on the internet and a representation provided by the manufacturer can be seen in Figure B.1. It has a good transmittance in the blue region around $400 \mathrm{~nm}$ and very low transmittance in the infrared around $800 \mathrm{~nm}$.

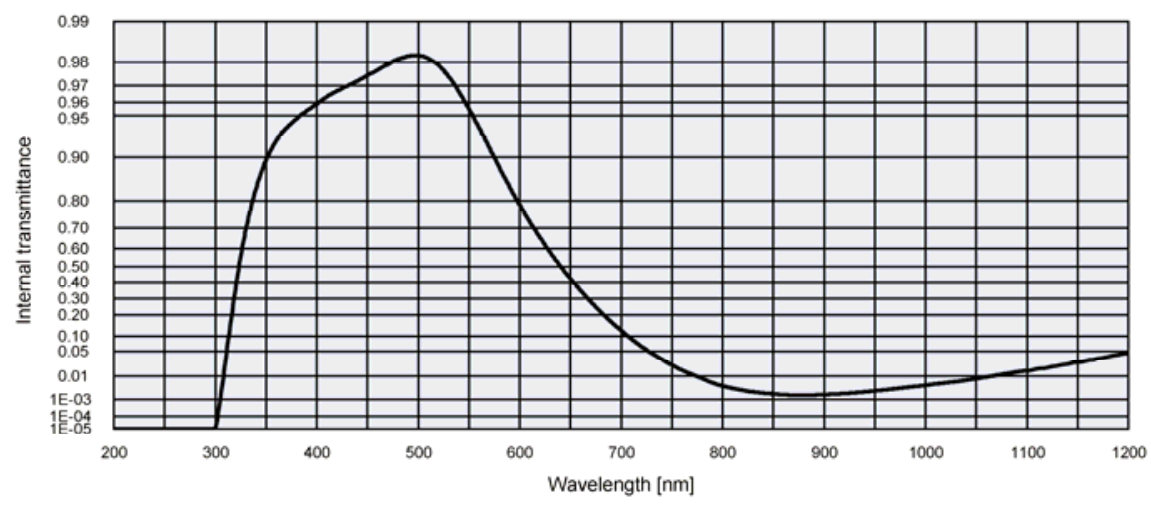

Figure B.1: Transmittance of the FGB37s filter given by the manufacturer

We used this filter to remove the unconverted fundamental in SHG (in Chapter 3, 4 and 7) and it was also used in the SC characterization (Chapter 6). However, only a few data from those 
represented in the previous figure were available, and were insufficient for correct application of the filter's transmittance to our experimental measurements. In Figure B.2 we plot the data that were available in our area of interest with filled red dots. Thus, we chose a cubic spline interpolation of the data; the results are represented by a blue line in the same figure.

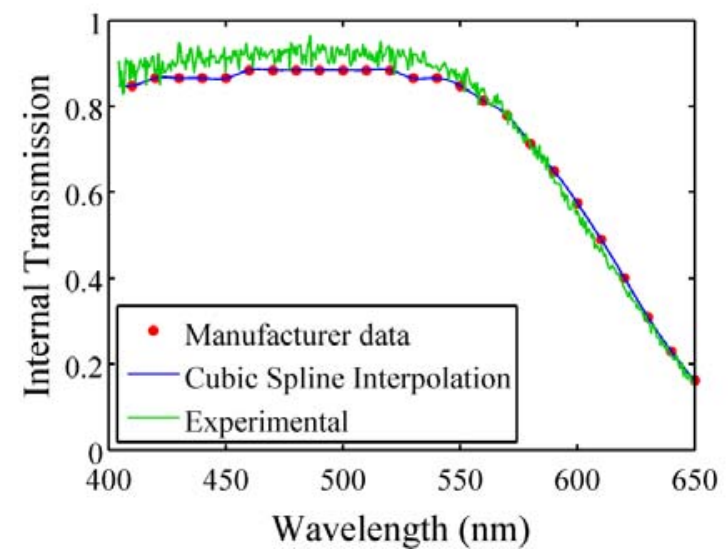

Figure B.2. Transmittance of the filter in the area of interest.

In addition, we made a measurement of the transmittance of the filter. To do so, we used a white light from a common bulb as reference and measured the transmittance of the filter with the help of the spectrometer (Avantes, AvaSpec-2048). It turned out that our experimental results, included in Figure B.2 (green line) matched the data given by the manufacturer fairly well.

\section{Cutoff Filter FES 0750}

This is an interferometric filter, different from the coloured glass described previously. These types of filter are created by depositing layers of low-index and high-index dielectric material onto a substrate. The transmitted or blocked wavelengths are determined by constructive or destructive interference, respectively. Further information can be found at the catalogue page: http://www.thorlabs.com/NewGroupPage9.cfm?ObjectGroup ID=918\&pn=FES0750\#3247

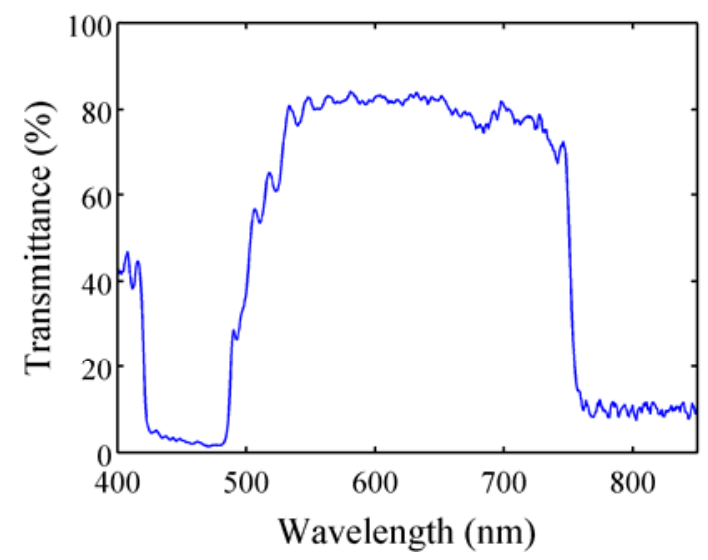

Figure B.3. Experimentally measured external transmittance of the FES0750 filter.

In the case of our filter it had a cut-off wavelength of $750 \mathrm{~nm}$, meaning that longer wavelengths would be blocked, and shorter ones would pass through. At the time of the experiments the 
transmittance data were not available on the company's web page, and hence we characterized the filter in the same way as we did in the previous case, calculating its external transmittance. We used the same spectrometer and a white-light lamp source as before. The result was the plot shown in Figure B.3.

As can be seen in the figure the transmittance of the filter is good in the visible range, from $\sim 550$ to $\sim 750 \mathrm{~nm}$, but when passing to lower wavelengths the transmittance starts to drop, reaching a region with zero transmittance in the blue spectral region. More recently, we have discovered that the data from the manufacturer were available in the manufacturer's web page. We have plotted these data in Figure B.4.

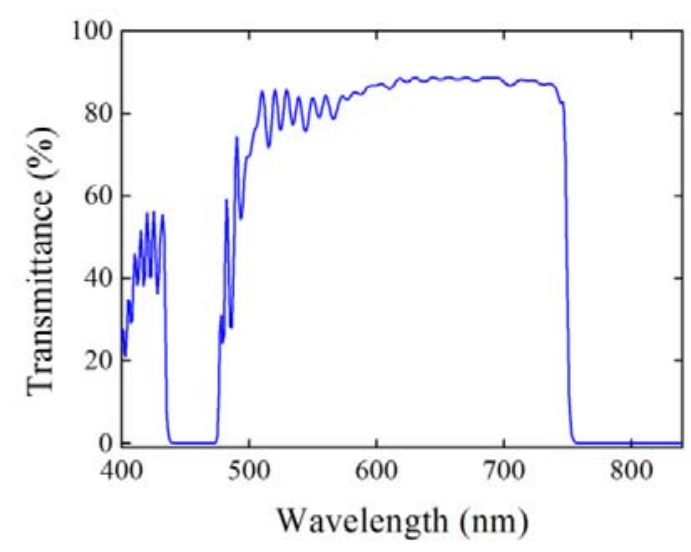

Figure B.4: Transmittance of the FES075 filter given by the company.

As can be seen in Figure B.4, the transmittance in the visible region is around $80 \%$, which approximately matches the experimentally measured value. Moreover there is a rejection zone, which corresponds to the wavelengths $(435-470 \mathrm{~nm})$ also seen in our measurements. 



\section{Appendix C}

\section{Laser Parameters}

In this appendix we present a summary of the parameters of the laser beam used in the experiments. As was mention in the introduction (Chapter 1), the laser we used was a regeneratively amplified Ti:Sapphire based system (Spitfire, Spectra Physics.). In the table below find summarized the characteristics of this laser given by the manufacturer:

\begin{tabular}{ll}
\hline Pulse duration & $120 \mathrm{fs}$ \\
Pulse Central Wavelength & $795 \mathrm{~nm}$ \\
Pulse Bandwidth (FWHM) & $9 \mathrm{~nm}$ \\
Pulse duration & $120 \mathrm{fs}$ \\
Pulse Energy & $1 \mathrm{~mJ}$ \\
Repetition rate & $1 \mathrm{kHz}$ \\
Beam Diameter (1/2 $\left.{ }^{2}\right)$ & $9 \mathrm{~mm}$ \\
Pre-Pulse Contrast Ratio & $>1000: 1$ \\
Post-Pulse Contrast Ratio & $>100: 1$ \\
Energy Stability & $<0.5 \% \mathrm{rms}$ over 24 hours \\
Beam Pointing Stability & $<20 \mu \mathrm{rad}^{\circ} \mathrm{C}$ \\
\hline Spatial Mode & $\mathrm{TEM}_{00}\left(\mathrm{M}^{2}>1.3\right.$ on both axes) \\
Polarization & Linear, Horizontal \\
\hline
\end{tabular}

We also have performed some measurements of the laser parameters relevant to our experiments: the spectrum of the laser, the pulses temporal profile and the beam spatial profile, all of them measured in our experimental table, after the laser had been propagated approximately 5 meters in air. 


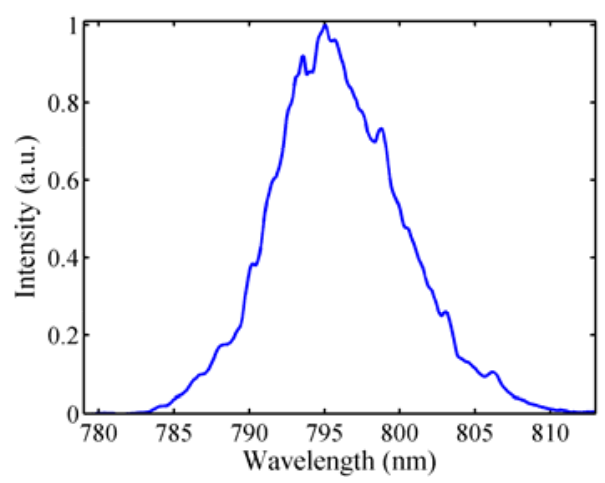

Figure C.1. Spectrum of the laser, measured with the spectrometer: AvaSpec-2048 (Avantes).
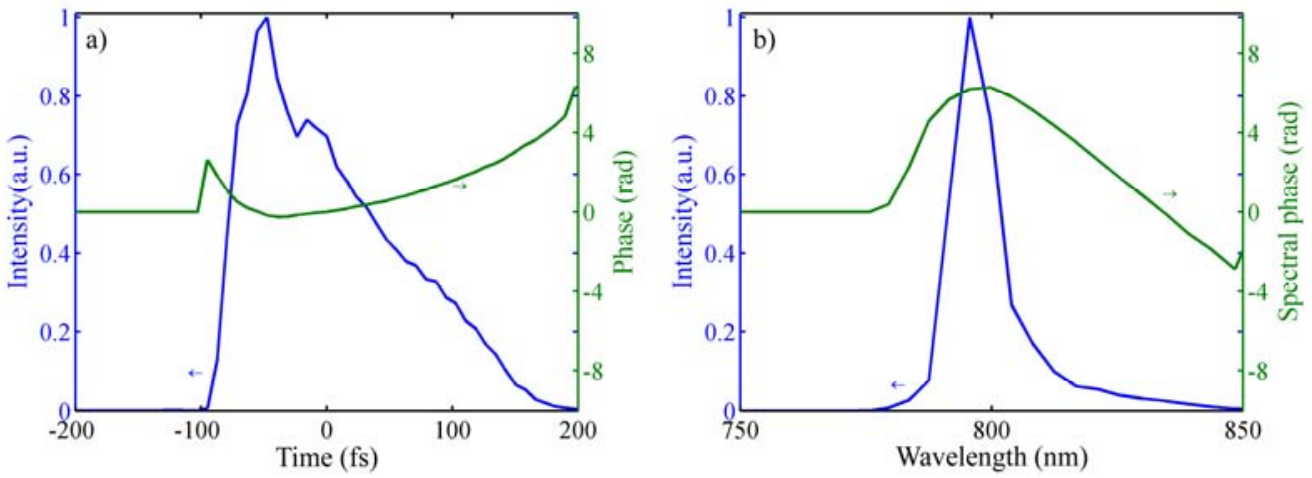

Figure C.2. Laser pulse intensity and phase a) time domain and b) frequency domain, taken with the Grenouille (Swamp Optics). The measurements are compatible with the pulse time duration offered by the manufacturer.

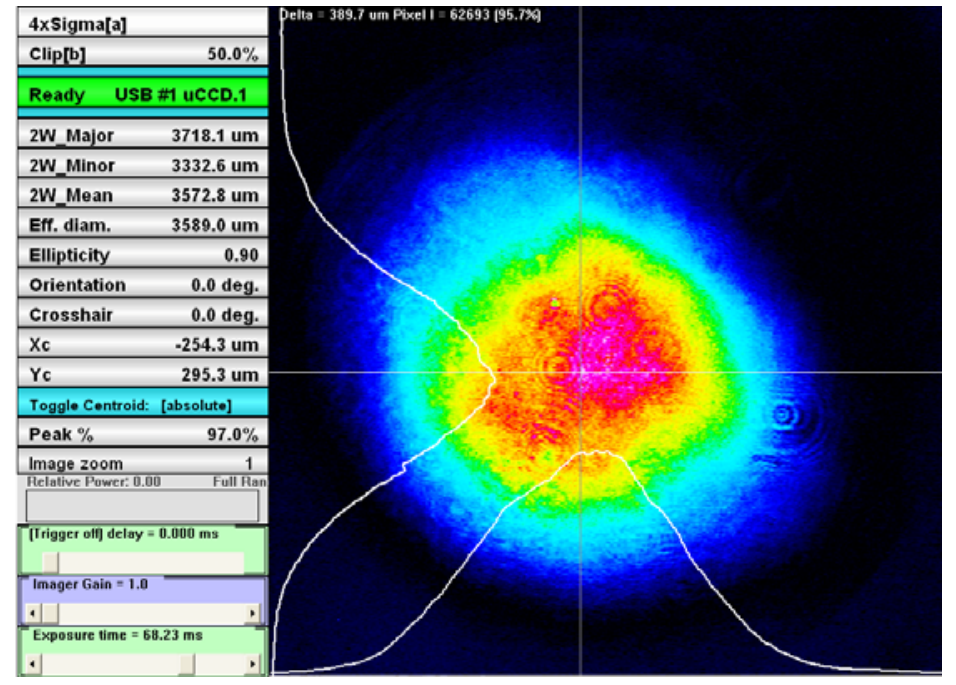

Figure C.3. CCD image of the laser beam profile taken with the camera WinCamD (DataRay). The image shows a fairly good spatial profile. The data in the picture have to be multiplied by 1.6 the factor of the taper used for taken the shot. This results in a beam diameter of $5.72 \mathrm{~mm}$ at $50 \%$ criterion and $8.58 \mathrm{~mm}$ at $1 / \mathrm{e}^{2}$ criterion. 


\section{Bibliography}

1. T. H. Maiman, "Stimulated optical radiation in ruby," Nature 187, 493-494 (1960).

2. B. Alonso, R. Borrego-Varillas, C. Hernández-García, J. A. Pérez-Hernández, and C. Romero, eds. El láser, la luz de nuestro tiempo (Salamanca, 2010).

3. P. A. Franken, G. Weinreich, C. W. Peters, and A. E. Hill, "Generation of optical harmonics," Physical Review Letters 7, 118-119 (1961).

4. "National Ignition Facility, NIF," https://lasers.llnl.gov/about/.

5. "Vulcan laser facility," http://www.clf.rl.ac.uk/Facilities/Vulcan/Vulcan+laser/12250.aspx.

6. A. E. Siegman, Lasers (University Science Books, Sausalito, 1985).

7. F. J. McClung, and R. W. Hellwarth, "Giant optical pulsations from ruby," Journal of Applied Physics 33, 828 (1962).

8. L. E. Hargrove, R. L. Fork, and M. A. Pollack, "Locking of He-Ne laser modes induced by synchronous intracavity modulation," Applied Physics Letters 5, 4-5 (1964).

9. C. V. Shank, and E. P. Ippen, "Subpicosecond Kilowatt pulses from a mode-locked CW dye laser," Applied Physics Letters 24, 373-375 (1974).

10. P. F. Moulton, "Spectroscopic and laser characteristics of Ti: $\mathrm{Al}_{2} \mathrm{O}_{3}$," Journal of the Optical Society of America B-Optical Physics 3, 125-133 (1986).

11. D. E. Spence, P. N. Kean, and W. Sibbett, "60-fs pulse generation from a self-mode-locked Ti:Sapphire laser," Optics Letters 16, $42-44$ (1991).

12. D. Strickland, and G. Mourou, "Compression of amplified chirped optical pulses," Optics Communications 56, 219-221 (1985).

13. J. Squier, F. Salin, G. Mourou, and D. Harter, "100-fs pulse generation and amplification in Ti: $\mathrm{Al}_{2} \mathrm{O}_{3}$," Optics Letters 16, 324-326 (1991).

14. "Centro de Lásers Pulsados (CLPU)," http://www.clpu.es/. 
15. A. Dubietis, G. Jonusauskas, and A. Piskarskas, "Powerful femtosecond pulse generation by chirped and stretched pulse parametric amplification in BBO crystal," Optics Communications 88, 437-440 (1992).

16. V. V. Lozhkarev, G. I. Freidman, V. N. Ginzburg, E. V. Katin, E. A. Khazanov, A. V. Kirsanov, G. A. Luchinin, A. N. Mal'shakov, M. A. Martyanov, O. V. Palashov, A. K. Poteomkin, A. M. Sergeev, A. A. Shaykin, and I. V. Yakovlev, "Compact 0.56 Petawatt laser system based on optical parametric chirped pulse amplification in KD*P crystals," Laser Physics Letters 4, 421-427 (2007).

17. A. Dubietis, R. Butkus, and A. P. Piskarskas, "Trends in chirped pulse optical parametric amplification," Ieee Journal of Selected Topics in Quantum Electronics 12, 163-172 (2006).

18. E. W. Gaul, M. Martinez, J. Blakeney, A. Jochmann, M. Ringuette, D. Hammond, T. Borger, R. Escamilla, S. Douglas, W. Henderson, G. Dyer, A. Erlandson, R. Cross, J. Caird, C. Ebbers, and T. Ditmire, "Demonstration of a 1.1 petawatt laser based on a hybrid optical parametric chirped pulse amplification/mixed Nd:glass amplifier," Applied Optics 49, 1676-1681 (2010).

19. M. E. Fermann, A. Galvanauskas, and G. Sucha, eds. Ultrafast lasers: Technology and applications (Marcel Dekker, New York, 2003).

20. S. W. Bahk, P. Rousseau, T. A. Planchon, V. Chvykov, G. Kalintchenko, A. Maksimchuk, G. A. Mourou, and V. Yanovsky, "Generation and characterization of the highest laser intensities $\left(10^{22} \mathrm{~W} / \mathrm{cm}^{2}\right), "$ Optics Letters 29, 2837-2839 (2004).

21. T. Brabec, and F. Krausz, "Intense few-cycle laser fields: Frontiers of nonlinear optics," Reviews of Modern Physics 72, 545-591 (2000).

22. M. Drescher, M. Hentschel, R. Kienberger, M. Uiberacker, V. Yakovlev, A. Scrinzi, T. Westerwalbesloh, U. Kleineberg, U. Heinzmann, and F. Krausz, "Time-resolved atomic inner-shell spectroscopy," Nature 419, 803-807 (2002).

23. C. Vozzi, E. Calegari, E. Ferrari, M. Lucchini, S. De Silvestri, O. Svelto, G. Sansone, S. Stagira, and M. Nisoli, "Advances in laser technology for isolated attosecond pulse generation," Laser Physics Letters 6, 259-267 (2009).

24. B. N. Chichkov, C. Momma, S. Nolte, F. vonAlvensleben, and A. Tunnermann, "Femtosecond, picosecond and nanosecond laser ablation of solids," Applied Physics A-Materials Science \& Processing 63, 109-115 (1996).

25. R. W. Boyd, Nonlinear Optics (Academic Press, San Diego, 2003).

26. A. Braun, G. Korn, X. Liu, D. Du, J. Squier, and G. Mourou, "Self-channeling of high-peak power femtosecond laser-pulses in air," Optics Letters 20, 73-75 (1995).

27. P. E. Powers, Fundamentals of nonlinear optics (CRC Press - Taylor and Francis Group, Boca Raton, 2011).

28. Y. R. Shen, The principles of non-linear optics (John Wiley \& Sons, New York, 1984).

29. M. Born, and E. Wolf, Principles of Optics (Cambridge University Press, 1999 (7th edition)).

30. J. D. Jackson, Classical electrodynamics (John Wiley \& Sons, New York, 1999). 
31. J. M. Cabrera, F. Agulló López, and F. Jesús López, Óptica Electromagnética (AdisonWesley/Universidad Autónoma de Madrid, Madrid, 2000).

32. S. E. Harris, M. K. Oshman, and R. L. Byer, "Observation of tunable optical parametric fluorescence," Physical Review Letters 18, 732 (1967).

33. J. A. Armstrong, N. Bloembergen, J. Ducuing, and P. S. Pershan, "Interactions between ligth waves in a nonlinear dielectric," Physical Review 127, 1918 (1962).

34. J. E. Midwinter, and J. Warner, "Effects of phase matching method and of uniaxial crystal symmetry on polar distribution of second-order non-linear optical polarization," British Journal of Applied Physics 16, 1135 (1965).

35. R. A. Baumgartner, and R. L. Byer, "Optical parametric amplification," Ieee Journal of Quantum Electronics 15, 432-444 (1979).

36. W. Brunner, and H. Paul, "Theory of Optical Parametric Amplification and Oscillation," in Progress in Optics, E. Wolf, ed. (North-Holland Publishing Companig, 1977).

37. J. Y. Zhang, J. Y. Huang, H. Wang, K. S. Wong, and G. K. Wong, "Second-harmonic generation from regeneratively amplified femtosecond laser pulses in BBO and LBO crystals," Journal of the Optical Society of America B-Optical Physics 15, 200-209 (1998).

38. P. L. Kelley, I. P. Kaminow, and G. P. Agrawal, eds. Nonlinear Fiber Optics (Academic Press, San Diego, 2001).

39. "SNLO Software," http://www.as-photonics.com/SNLO.html.

40. X. Gu, S. Akturk, and R. Trebino, "Spatial chirp in ultrafast optics," Optics Communications 242, 599-604 (2004).

41. D. Eimerl, L. Davis, S. Velsko, E. K. Graham, and A. Zalkin, "Optical, mechanical and thermal properties of barium borate," Journal of Applied Physics 62, 1968-1983 (1987).

42. R. Trebino, Frequency-resolved optical gating: The measurement of ultrashort laser pulses (Kluwer Academic Publishers, Norwell, 2000).

43. J. Y. Zhang, A. P. Shreenath, M. Kimmel, E. Zeek, R. Trebino, and S. Link, "Measurement of the intensity and phase of attojoule femtosecond light pulses using optical-parametricamplification cross-correlation frequency-resolved optical gating," Optics Express 11, 601-609 (2003).

44. A. Furbach, T. Le, C. Spielmann, and F. Krausz, "Generation of 8-fs pulses at $390 \mathrm{~nm}$," Applied Physics B-Lasers and Optics 70, S37-S40 (2000).

45. R. Trebino, K. W. DeLong, D. N. Fittinghoff, J. N. Sweetser, M. A. Krumbugel, B. A. Richman, and D. J. Kane, "Measuring ultrashort laser pulses in the time-frequency domain using frequency-resolved optical gating," Review of Scientific Instruments 68, 3277-3295 (1997).

46. $\quad$ "FROG 3 Software," http://www.femtosoft.biz/functionality.shtml.

47. P. O'Shea, M. Kimmel, X. Gu, and R. Trebino, "Highly simplified device for ultrashortpulse measurement," Optics Letters 26, 932-934 (2001). 
48. A. P. Baronavski, H. D. Ladouceur, and J. K. Shaw, "Analysis of cross-correlation, phasevelocity mismatch, and gruop-velocity mismatches in sum-frequency generation," IEEE Journal of Quantum Electronics 29, 580-589 (1993).

49. C. Mendez, J. R. V. De Aldana, G. A. Torchia, and L. Roso, "Optical waveguide arrays induced in fused silica by void-like defects using femtosecond laser pulses," Applied Physics BLasers and Optics 86, 343-346 (2007).

50. F. Dausinger, F. Lichtner, and H. Lubatschowski, eds. Femtosecond technology for technical and medical applications (Springer-Verlag, Berlin, 2004).

51. A. W. Miziolek, V. Palleschi, and I. Schechter, eds. Laser induced breakdown spectroscopy. Fundamentals and applications (Cambridge University Press, 2006).

52. M. Galceran, M. C. Pujol, C. Mendez, A. Garcia, P. Moreno, L. Roso, M. Aguilo, and F. Diaz, "Synthesis of monoclinic $\mathrm{KGd}\left(\mathrm{WO}_{4}\right)_{2}$ nanocrystals by two preparation methods," Journal of Nanoparticle Research 11, 717-724 (2009).

53. N. Takeshima, Y. Narita, S. Tanaka, Y. Kuroiwa, and K. Hirao, "Fabrication of highefficiency diffraction gratings in glass," Optics Letters 30, 352-354 (2005).

54. Y. Li, P. Lu, N. Dai, X. Wang, Y. Wang, B. Yu, and H. Long, "Surface relief diffraction gratings written on beta-BaB2O4 crystal by femtosecond pulses," Applied Physics B-Lasers and Optics 88, 227-230 (2007).

55. C. Mendez, J. R. V. de Aldana, G. A. Torchia, and L. Roso, "Integrated-grating-induced control of second-harmonic beams in frequency-doubling crystals," Optics Letters 30, 2763-2765 (2005).

56. G. A. Torchia, C. Mendez, I. Arias, L. Roso, A. Rodenas, and D. Jaque, "Laser gain in femtosecond microstructured Nd : MgO : LiNbO3 crystals," Applied Physics B-Lasers and Optics 83, 559-563 (2006).

57. T. R. Zhang, H. R. Choo, and M. C. Downer, "Phase and group-velocity matching for second harmonic generation of femtosecond pulses," Applied Optics 29, 3927-3933 (1990).

58. C. Radzewicz, Y. B. Band, G. W. Pearson, and J. S. Krasinski, "Short-pulse nonlinear frequency conversion without group velocity mismatch broadening," Optics Communications 117, 295-302 (1995).

59. A. V. Smith, "Group-velocity-matched three-wave mixing in birefringent crystals," Optics Letters 26, 719-721 (2001).

60. O. E. Martinez, "Pulse distortions in tilted pulse schemes for ultrashort pulses," Optics Communications 59, 229-232 (1986).

61. M. M. Fejer, G. A. Magel, D. H. Jundt, and R. L. Byer, "Quasi-phase-matched second harmonic generation: Tuning and tolerances," Ieee Journal of Quantum Electronics 28, 2631-2654 (1992).

62. M. Baudrier-Raybaut, R. Haidar, P. Kupecek, P. Lemasson, and E. Rosencher, "Random quasi-phase-matching in bulk polycrystalline isotropic nonlinear materials," Nature 432, 374-376 (2004). 
63. V. Roppo, W. Wang, K. Kalinowski, Y. Kong, C. Cojocaru, J. Trull, R. Vilaseca, M. Scalora, W. Krolikowski, and Y. Kivshar, "The role of ferroelectric domain structure in second harmonic generation in random quadratic media," Optics Express 18, 4012-4022 (2010).

64. V. Roppo, D. Dumay, J. Trull, C. Cojocaru, S. M. Saltiel, K. Staliunas, R. Vilaseca, D. N. Neshev, W. Krolikowski, and Y. S. Kivshar, "Planar second-harmonic generation with noncollinear pumps in disordered media," Optics Express 16, 14192-14199 (2008).

65. V. I. Klimov, and D. W. McBranch, "Femtosecond high-sensitivity, chirp-free transient absorption spectroscopy using kilohertz lasers," Optics Letters 23, 277-279 (1998).

66. E. Pontecorvo, S. M. Kapetanaki, M. Badioli, D. Brida, M. Marangoni, G. Cerullo, and T. Scopigno, "Femtosecond stimulated Raman spectrometer in the 320-520nm range," Optics Express 19, 1107-1112 (2011).

67. M. Oujja, A. Garcia, C. Romero, J. R. V. de Aldana, P. Moreno, and M. Castillejo, "UV laser removal of varnish on tempera paints with nanosecond and femtosecond pulses," Physical Chemistry Chemical Physics 13, 4625-4631 (2011).

68. S. Cavalieri, L. Fini, E. Sali, and R. Buffa, "Enhancement of harmonic generation by Fresnel-lensing effects," Optics Letters 31, 1298-1300 (2006).

69. C. A. Yang, K. B. Shi, H. F. Li, Q. A. Xu, V. Gopalan, and Z. W. Liu, "Chromatic second harmonic imaging," Optics Express 18, 23837-23843 (2010).

70. E. Hecht, Optica (Addisson Wesley Iberoamericana, Madrid, 2000).

71. V. Moreno, J. F. Roman, and J. R. Salgueiro, "High efficiency diffractive lenses: Deduction of kinoform profile," American Journal of Physics 65, 556-562 (1997).

72. G. Minguez-Vega, O. Mendoza-Yero, E. Tajahuerce, J. Lancis, and P. Andres, "Optical Filter Based on a Spatially Patterned Kinoform Diffractive Lens," Ieee Photonics Technology Letters 21, 347-349 (2009).

73. L. B. Lesem, P. M. Hirsch, and J. A. Jordan, "Kinofrom: a new wavefront reconstruction device," IBM Journal of Research and Development 13, 150 (1969).

74. Z. Bor, "Distortion of femtosecond laser pulses in lenses," Optics Letters 14, 119-121 (1989).

75. R. Ashman, and M. Gu, "Effect of ultrashort pulsed illumination on foci caused by a Fresnel zone plate," Applied Optics 42, 1852-1855 (2003).

76. G. Minguez-Vega, C. Romero, O. Mendoza-Yero, J. R. V. de Aldana, R. Borrego-Varillas, C. Mendez, P. Andres, J. Lancis, V. Climent, and L. Roso, "Wavelength tuning of femtosecond pulses generated in nonlinear crystals by using diffractive lenses," Optics Letters 35, 3694-3696 (2010).

77. G. D. Boyd, and D. A. Kleinman, "Parametric interaction of focused gaussian light beams," Journal of Applied Physics 39, 3597 (1968).

78. R. Borrego-Varillas, C. Romero, J. R. Vázquez de Aldana, J. M. Bueno, and L. Roso, "Wavefront retrieval of amplified femtosecond beams by second-harmonic generation," Optics Express 19, 22851-22862 (2011). 
79. S. Velghe, J. Primot, N. Guerineau, M. Cohen, and B. Wattellier, "Wave-front reconstruction from multidirectional phase derivatives generated by multilateral shearing interferometers," Optics Letters 30, 245-247 (2005).

80. F. Rotermund, and V. Petrov, "Generation of the fourth harmonic of a femtosecond Ti : sapphire laser," Optics Letters 23, 1040-1042 (1998).

81. R. R. Alfano, and S. L. Shapiro, "Emission in region 4000 to 7000 A via four-photon coupling in glass," Physical Review Letters 24, 584 (1970).

82. R. R. Alfano, The supercontinuum laser source (Springer, New York, 2006).

83. P. B. Corkum, C. Rolland, and T. Srinivasanrao, "Supercontinuum generation in gases," Physical Review Letters 57, 2268-2271 (1986).

84. A. Brodeur, and S. L. Chin, "Ultrafast white-light continuum generation and self-focusing in transparent condensed media," Journal of the Optical Society of America B-Optical Physics 16, 637-650 (1999).

85. R. L. Fork, C. V. Shank, C. Hirlimann, R. Yen, and W. J. Tomlinson, "Femtosecond whitelight continuum pulses," Optics Letters 8, 1-3 (1983).

86. R. A. Negres, J. M. Hales, D. J. Hagan, and E. W. Van Stryland, "Experiment and analysis of two-photon absorption spectroscopy using a white-light continuum probe," IEEE Journal of Quantum Electronics 38, 1205-1216 (2002).

87. Z. W. Wilkes, S. Varma, Y. H. Chen, H. M. Milchberg, T. G. Jones, and A. Ting, "Direct measurements of the nonlinear index of refraction of water at 815 and $407 \mathrm{~nm}$ using single-shot supercontinuum spectral interferometry," Applied Physics Letters 94, 211102 (2009).

88. O. Varela, A. Zair, J. San Roman, B. Alonso, I. J. Sola, C. Prieto, and L. Roso, "Abovemillijoule super-continuum generation using polarisation dependent filamentation in atoms and molecules," Optics Express 17, 3630-3639 (2009).

89. G. Cerullo, and S. De Silvestri, "Ultrafast optical parametric amplifiers," Review of Scientific Instruments 74, 1-18 (2003).

90. C. Romero, R. Borrego-Varillas, A. Camino, G. Minguez-Vega, O. Mendoza-Yero, J. Hernandez-Toro, and J. R. V. de Aldana, "Diffractive optics for spectral control of the supercontinuum generated in sapphire with femtosecond pulses," Optics Express 19, 4977-4984 (2011).

91. M. Sheikbahae, A. A. Said, T. H. Wei, D. J. Hagan, and E. W. Vanstryland, "Sensitive measurement of optical nonlinearities using a single beam," IEEE Journal of Quantum Electronics 26, 760-769 (1990).

92. A. Major, F. Yoshino, I. Nikolakakos, J. S. Aitchison, and P. W. E. Smith, "Dispersion of the nonlinear refractive index in sapphire," Optics Letters 29, 602-604 (2004).

93. E. T. J. Nibbering, G. Grillon, M. A. Franco, B. S. Prade, and A. Mysyrowicz, "Determination of the inertial contribution to the nonlinear refractive index of air, $\mathrm{N}_{2}$, and $\mathrm{O}_{2}$ by use of unfocused high-intensity femtosecond laser pulses," Journal of the Optical Society of America B-Optical Physics 14, 650-660 (1997). 
94. C. Nagura, A. Suda, H. Kawano, M. Obara, and K. Midorikawa, "Generation and characterization of ultrafast white-light continuum in condensed media," Applied Optics 41, 3735 3742 (2002).

95. E. T. J. Nibbering, P. F. Curley, G. Grillon, B. S. Prade, M. A. Franco, F. Salin, and A. Mysyrowicz, "Conical emission from self-guided femtosecond pulses in air," Optics Letters 21, 6264 (1996).

96. J. H. Marburger, "Self-focusing: Theory (Reprinted from vol 4, pg 35-110, 1975)," Selffocusing: Past and Present 114, 25-+ (2009).

97. J. E. Rothenberg, "Space-time focusing: breakdown of the slowly varying envelope approximation in the self-focusing of femtosecond pulses," Optics Letters 17, 1340-1342 (1992).

98. S. S. Mao, F. Quere, S. Guizard, X. Mao, R. E. Russo, G. Petite, and P. Martin, "Dynamics of femtosecond laser interactions with dielectrics," Applied Physics a-Materials Science \& Processing 79, 1695-1709 (2004).

99. A. Couairon, and A. Mysyrowicz, "Femtosecond filamentation in transparent media," Physics Reports-Review Section of Physics Letters 441, 47-189 (2007).

100. J. B. Ashcom, R. R. Gattass, C. B. Schaffer, and E. Mazur, "Numerical aperture dependence of damage and supercontinuum generation from femtosecond laser pulses in bulk fused silica," Journal of the Optical Society of America B-Optical Physics 23, 2317-2322 (2006).

101. A. Brodeur, and S. L. Chin, "Band-gap dependence of the ultrafast white-light continuum," Physical Review Letters 80, 4406-4409 (1998).

102. M. Bradler, P. Baum, and E. Riedle, "Femtosecond continuum generation in bulk laser host materials with sub-mu J pump pulses," Applied Physics B-Lasers and Optics 97, 561-574 (2009).

103. X. H. Ni, C. Wang, X. C. Liang, M. Al-Rubaiee, and R. R. Alfano, "Fresnel diffraction supercontinuum generation," IEEE Journal of Selected Topics in Quantum Electronics 10, 12291232 (2004).

104. V. Kartazaev, and R. R. Alfano, "Polarization properties of SC generated in $\mathrm{CaF}_{2}$," Optics Communications 281, 463-468 (2008).

105. X. J. Fang, and T. Kobayashi, "Evolution of a super-broadened spectrum in a filament generated by an ultrashort intense laser pulse in fused silica," Applied Physics B-Lasers and Optics 77, 167-170 (2003).

106. J. J. Yang, and G. G. Mu, "Multi-dimensional observation of white-light filaments generated by femtosecond laser pulses in condensed medium," Optics Express 15, 4943-4952 (2007).

107. R. Borrego-Varillas, C. Romero, O. Mendoza-Yero, G. Mínguez-Vega, and J. R. Vázquez de Aldana, "Supercontinuum visible femtosecond pulses generated in sapphire with diffractive lenses," (In preparation).

108. A. Kummrow, M. Wittmann, F. Tschirschwitz, G. Korn, and E. T. J. Nibbering, "Femtosecond ultraviolet pulses generated using noncollinear optical parametric amplification and sum frequency mixing," Applied Physics B-Lasers and Optics 71, 885-887 (2000). 
109. F. Rotermund, and V. Petrov, "Mercury thiogallate mid-infrared femtosecond optical parametric generator pumped at $1.25 \mathrm{mu} \mathrm{m}$ by a $\mathrm{Cr}$ : forsterite regenerative amplifier," Optics Letters 25, 746-748 (2000).

110. A. Shirakawa, I. Sakane, M. Takasaka, and T. Kobayashi, "Sub-5-fs visible pulse generation by pulse-front-matched noncollinear optical parametric amplification," Applied Physics Letters 74, 2268-2270 (1999).

111. M. Nisoli, S. Stagira, S. De Silvestri, O. Svelto, G. Valiulis, and A. Varanavicius, "Parametric generation of high-energy 14.5-fs light pulses at $1.5 \mathrm{mu}$ m," Optics Letters 23, 630-632 (1998).

112. A. Baltuska, T. Fuji, and T. Kobayashi, "Visible pulse compression to 4 fs by optical parametric amplification and programmable dispersion control," Optics Letters 27, 306-308 (2002).

113. A. Baltuska, T. Fuji, and T. Kobayashi, "Controlling the carrier-envelope phase of ultrashort light pulses with optical parametric amplifiers," Physical Review Letters 88 (2002).

114. R. Weigand, J. T. Mendonca, and H. M. Crespo, "Cascaded nondegenerate four-wavemixing technique for high-power single-cycle pulse synthesis in the visible and ultraviolet ranges," Physical Review A 79 (2009).

115. J. L. Silva, R. Weigand, and H. M. Crespo, "Octave-spanning spectra and pulse synthesis by nondegenerate cascaded four-wave mixing," Optics Letters 34, 2489-2491 (2009).

116. M. Nisoli, S. DeSilvestri, O. Svelto, R. Szipocs, K. Ferencz, C. Spielmann, S. Sartania, and F. Krausz, "Compression of high-energy laser pulses below 5 fs," Optics Letters 22, 522-524 (1997).

117. E. Goulielmakis, S. Koehler, B. Reiter, M. Schultze, A. J. Verhoef, E. E. Serebryannikov, A. M. Zheltikov, and R. Krausz, "Ultrabroadband, coherent light source based on self-channeling of few-cycle pulses in helium," Optics Letters 33, 1407-1409 (2008).

118. O. Varela, B. Alonso, I. J. Sola, J. San Roman, A. Zair, C. Mendez, and L. Roso, "Selfcompression controlled by the chirp of the input pulse," Optics Letters 35, 3649-3651 (2010).

119. I. N. Ross, P. Matousek, M. Towrie, A. J. Langley, and J. L. Collier, "The prospects for ultrashort pulse duration and ultrahigh intensity using optical parametric chirped pulse amplifiers," Optics Communications 144, 125-133 (1997).

120. D. Brida, C. Manzoni, G. Cirmi, M. Marangoni, S. De Silvestri, and G. Cerullo, "Generation of broadband mid-infrared pulses from an optical parametric amplifier," Optics Express 15, 15035-15040 (2007).

121. C. Manzoni, C. Vozzi, E. Benedetti, G. Sansone, S. Stagira, O. Svelto, S. De Silvestri, M. Nisoli, and G. Cerullo, "Generation of high-energy self-phase-stabilized pulses by differencefrequency generation followed by optical parametric amplification," Optics Letters 31, 963-965 (2006).

122. J. Limpert, C. Aguergaray, S. Montant, I. Manek-Honninger, S. Petit, D. Descamps, E. Cormier, and F. Salin, "Ultra-broad bandwidth parametric amplification at degeneracy," Optics Express 13, 7386-7392 (2005). 
123. G. Cerullo, M. Nisoli, S. Stagira, and S. De Silvestri, "Sub-8-fs pulses from an ultrabroadband optical parametric amplifier in the visible," Optics Letters 23, 1283-1285 (1998).

124. V. Krylov, O. Ollikainen, J. Gallus, U. Wild, A. Rebane, and A. Kalintsev, "Efficient noncollinear parametric amplification of weak femtosecond pulses in the visible and near-infrared spectral range," Optics Letters 23, 100-102 (1998).

125. R. Huber, H. Satzger, W. Zinth, and J. Wachtveitl, "Noncollinear optical parametric amplifiers with output parameters improved by the application of a white light continuum generated in $\mathrm{CaF}_{2}$," Optics Communications 194, 443-448 (2001).

126. J. C. Diels, and W. Rudolph, Ultrashort Laser Pulse Phenomena (Academic Press, Albuquerque, 2006).

127. L. Hongjun, Z. Wei, C. Guofu, W. Yishan, C. Zhao, and R. Chi, "Investigation of spectral bandwidth of optical parametric amplification," Applied Physics B-Lasers and Optics 79, 569-576 (2004).

128. A. M. Siddiqui, G. Cirmi, D. Brida, F. X. Kartner, and G. Cerullo, "Generation of $<7$ fs pulses at $800 \mathrm{~nm}$ from a blue-pumped optical parametric amplifier at degeneracy," Optics Letters 34, 3592-3594 (2009).

129. T. J. Driscoll, G. M. Gale, and F. Hache, "Ti:Sapphire Second-Harmonic-Pumped visible range femtosecond optical parametric oscillator," Optics Communications 110, 638-644 (1994).

130. T. Wilhelm, J. Piel, and E. Riedle, "Sub-20-fs pulses tunable across the visible from a bluepumped single-pass noncollinear parametric converter," Optics Letters 22, 1494-1496 (1997).

131. E. Riedle, M. Beutter, S. Lochbrunner, J. Piel, S. Schenk1, S. Sporlein, and W. Zinth, "Generation of 10 to $50 \mathrm{fs}$ pulses tunable through all of the visible and the NIR," Applied Physics B-Lasers and Optics 71, 457-465 (2000).

132. G. Cerullo, M. Nisoli, and S. De Silvestri, "Generation of $11 \mathrm{fs}$ pulses tunable across the visible by optical parametric amplification," Applied Physics Letters 71, 3616-3618 (1997).

133. R. Danielius, A. Piskarskas, A. Stabinis, G. P. Banfi, P. Ditrapani, and R. Righini, "Traveling-wave parametric generation of widely tunable, highly coherent femtosecond lightpulses," Journal of the Optical Society of America B-Optical Physics 10, 2222-2232 (1993).

134. "Light Conversion Webpage," http://www.lightcon.com/.

135. V. Krylov, A. Kalintsev, A. Rebane, D. Erni, and U. P. Wild, "Noncollinear parametric generation in $\mathrm{LiIO}_{3}$ and b-barium borate by frequency-doubled femtosecond Ti:Sapphire laser-pulses," Optics Letters 20, 151-153 (1995).

136. T. G. Giallore, and C. L. Tang, "Quantum theory of spontaneous parametric scattering of intense light," Physical Review 166, 225-233 (1968).

137. V. Krylov, J. Gallus, U. P. Wild, A. Kalintsev, and A. Rebane, "Femtosecond noncollinear and collinear parametric generation and amplification in BBO crystal," Applied Physics B-Lasers and Optics 70, 163-168 (2000). 
138. T. Q. Jia, H. X. Chen, M. Huang, F. L. Zhao, X. X. Li, S. Z. Xu, H. Y. Sun, D. H. Feng, C. B. Li, X. F. Wang, R. X. Li, Z. Z. Xu, X. K. He, and H. Kuroda, "Ultraviolet-infrared femtosecond laser-induced damage in fused silica and $\mathrm{CaF}_{2}$ crystals," Physical Review B 73 (2006).

139. A. Baltuska, T. Fuji, and T. Kobayashi, "Self-referencing of the carrier-envelope slip in a 6fs visible parametric amplifier," Optics Letters 27, 1241-1243 (2002).

140. J. Y. Zhang, C. K. Lee, J. Y. Huang, and C. L. Pan, "Sub femto-joule sensitive single-shot OPA-XFROG and its application in study of white-light supercontinuum generation," Optics Express 12, 574-581 (2004).

141. "Centre des Laseres Intenses et Applications (CELIA)," http://www.celia.ubordeaux1.fr/?lang=fr.

142. F. Raoult, A. C. L. Boscheron, D. Husson, C. Sauteret, A. Modena, V. Malka, F. Dorchies, and A. Migus, "Efficient generation of narrow-bandwidth picosecond pulses by frequency doubling of femtosecond chirped pulses," Optics Letters 23, 1117-1119 (1998).

143. G. Veitas, and R. Danielius, "Generation of narrow-bandwidth tunable picosecond pulses by difference-frequency mixing of stretched pulses," Journal of the Optical Society of America BOptical Physics 16, 1561-1565 (1999).

144. Y. Kitagawa, H. Fujita, R. Kodama, H. Yoshida, S. Matsuo, T. Jitsuno, T. Kawasaki, H. Kitamura, T. Kanabe, S. Sakabe, K. Shigemori, N. Miyanaga, and Y. Izawa, "Prepulse-free petawatt laser for a fast ignitor," IEEE Journal of Quantum Electronics 40, 281-293 (2004).

145. B. C. Stuart, M. D. Feit, A. M. Rubenchik, B. W. Shore, and M. D. Perry, "Laser-induced damage in dielectrics with nanosecond to subpicosecond pulses," Physical Review Letters 74, 2248-2251 (1995).

146. G. Olivie, D. Giguere, F. Vidal, T. Ozaki, J. C. Kieffer, O. Nada, and I. Brunette, "Wavelength dependence of femtosecond laser ablation threshold of corneal stroma," Optics Express 16, 4121-4129 (2008).

147. T. H. Her, R. J. Finlay, C. Wu, S. Deliwala, and E. Mazur, "Microstructuring of silicon with femtosecond laser pulses," Applied Physics Letters 73, 1673-1675 (1998).

148. T. Baldacchini, J. E. Carey, M. Zhou, and E. Mazur, "Superhydrophobic surfaces prepared by microstructuring of silicon using a femtosecond laser," Langmuir 22, 4917-4919 (2006).

149. M. H. M. Halbwax, T. Sarnet, P. Delaporte, A. Sentis, H. Etienne, F. Torregrosa, V. Vervisch, I. Perichaud, and S. Martinuzzi, "Micro and nano-structuration of silicon by femtosecond laser: Application to silicon photovoltaic cells fabrication," Thin Solid Films 516, 6791-6795 (2008).

150. Y. Chen, and A. Vertes, "Adjustable fragmentation in laser desorption/ionization from laser-induced silicon microcolumn arrays," Analytical Chemistry 78, 5835-5844 (2006).

151. M. Y. Shen, C. H. Crouch, J. E. Carey, and E. Mazur, "Femtosecond laser-induced formation of submicrometer spikes on silicon in water," Applied Physics Letters 85, 5694-5696 (2004).

152. M. Castillejo, M. Martin, M. Oujja, D. Silva, R. Torres, A. Manousaki, V. Zafiropulos, O. F. van den Brink, R. M. A. Heeren, R. Teule, A. Silva, and H. Gouveia, "Analytical study of the 
chemical and physical changes induced by $\mathrm{KrF}$ laser cleaning of tempera paints," Analytical Chemistry 74, 4662-4671 (2002).

153. K. M. Davis, K. Miura, N. Sugimoto, and K. Hirao, "Writing waveguides in glass with a femtosecond laser," Optics Letters 21, 1729-1731 (1996).

154. A. Benayas, W. F. Silva, C. Jacinto, E. Cantelar, J. Lamela, F. Jaque, J. R. Vázquez de Aldana, G. A. Torchia, L. Roso, A. A. Kaminskii, and D. Jaque, "Thermally resistant waveguides fabricated in Nd:YAG ceramics by crossing femtosecond damage filaments," Optics Letters 35, 330-332 (2010).

155. W. F. Silva, C. Jacinto, A. Benayas, J. R. Vázquez de Aldana, G. A. Torchia, F. Chen, Y. Tan, and D. Jaque, "Femtosecond-laser-written, stress-induced Nd:YVO(4) waveguides preserving fluorescence and Raman gain," Optics Letters 35, 916-918 (2010).

156. N. N. Dong, Y. Tan, A. Benayas, J. R. Vázquez de Aldana, D. Jaque, C. Romero, F. Chen, and Q. M. Lu, "Femtosecond laser writing of multifunctional optical waveguides in a Nd:YVO(4)+KTP hybrid system," Optics Letters 36, 975-977 (2011).

157. N. N. Dong, J. M. de Mendivil, E. Cantelar, G. Lifante, J. R. Vázquez de Aldana, G. A. Torchia, F. Chen, and D. Jaque, "Self-frequency-doubling of ultrafast laser inscribed neodymium doped yttrium aluminum borate waveguides," Applied Physics Letters 98 (2011).

158. V. G. Dmitriev, G. G. Gurzadyan, and D. N. Nikogosyan, Handbook of Nonlinear Crystals (Springer, 1997 (Second ed.)). 